\title{
In silico reassignment of (+)-diplopyrone by NMR calculations. Use of a DP4/J-DP4/DP4+/DIP tandem to revise both relative and absolute configuration
}

\author{
Ariel M. Sarotti* \\ Instituto de Química Rosario (CONICET-UNR), Facultad de Ciencias Bioquímicas y Farmacéuticas, Universidad Nacional de Rosario, Suipacha 531, \\ Rosario 2000, Argentina. \\ sarotti@iquir-conicet.gov.ar
}

\section{List of contents}

1- Computational details. Pages S2-S3.

2- Most stable conformations found for compounds 1-8, $(R)$-MPTA-3, $(R)$-MPTA-4, $(S)$-MPTA-3 and $(S)$-MPTA-4 at the $\mathrm{PCM} / \mathrm{mPW} 1 \mathrm{PW} 91 / 6-31+\mathrm{G}^{* * / / B} 3 \mathrm{LYP} / 6-31 \mathrm{G}^{*}$ level of theory. Pages S4-S-6.

3- DP4 results of compounds 1-8. S7-S8.

4- $J$-DP4 results of compounds 1-8. Pages S8-S10.

5- DP4+ results of compounds 1-8. Pages S11-S12.

6- DP4/DP4+ results of compounds 1-8 obtained after fitting. Pages S13-S16.

7- ECD spectra of compounds 1-8 computed at the PCM/B3LYP/6-31G* level. Page S17.

8- DP4+ results of compounds ( $R$-MPTA-3, $(R)$-MPTA-4, $(S)$-MPTA-3 and (S)-MPTA-4. Pages S18-S20.

9- Cartesian coordinates of all compounds under study. Pages S21-S86. 
Computational Methods. All the quantum mechanical calculations were performed using Gaussian 09. ${ }^{1}$ For DP4 analysis, systematic conformational searches were done for each compound in the gas phase using the MMFF force field implemented in Macromodel. ${ }^{2}$ All conformers found within 10 $\mathrm{kcal} / \mathrm{mol}$ of the corresponding lowest energy conformer were subjected to GIAO NMR calculations at the B3LYP/6-31G** level of theory. ${ }^{3}$ For $J$-DP4 analysis, the full set of conformations found in the previous step was was narrowed down using the experimental $J_{4 \mathrm{a}-8 \mathrm{a}}(2.8 \mathrm{~Hz})$ and $J_{6-9}(4.2 \mathrm{~Hz})$ values repoted for ND to remove unsuitable conformations following the reported procedure. ${ }^{4}$ The remaining conformations in agreement with the experimental ${ }^{3} J_{\mathrm{HH}}$ values were submitted to $J$ coupling calculations at the B3LYP/6-31G** considering the Fermi contact term (FConly). For DP4+ analysis, ${ }^{5}$ the MMFF geometries were first optimized at the B3LYP/6-31G* level. Frequency calculations were done for all optimized geometries at the B3LYP/6-31G* level to determine the nature of the stationary point found, and to compute the thermochemical properties (at $1 \mathrm{~atm}$ and $298.15 \mathrm{~K}$ ). After removing duplicates, all the B3LYP/6-31G* optimized geometries were next subjected to GIAO NMR calculations at the mPW1PW91/6-31+G** level of theory, using the polarizable continuum model, PCM, ${ }^{6}$ with chloroform as the solvent. In all cases, the unscaled chemical shifts $\left(\delta_{u}\right)$ were computed using TMS as reference standard according to $\delta_{\mathrm{u}}=\sigma_{0}-\sigma_{\mathrm{x}}$, where $\sigma_{\mathrm{x}}$ is the Boltzmann averaged shielding tensor (over all significantly populated conformations) and $\sigma_{0}$ is the shielding tensor of TMS computed at the same level of theory employed for $\sigma_{\mathrm{x}}$. The Boltzmann averaging was done according to eq 1 :

$$
\sigma^{\mathrm{x}}=\frac{\sum_{i} \sigma_{i}^{\mathrm{x}} \mathrm{e}^{\left(-\mathrm{E}_{i} / \mathrm{RT}\right)}}{\sum_{i} \mathrm{e}^{\left(-\mathrm{E}_{i} / \mathrm{RT}\right)}}
$$

where $\sigma_{i}^{x}$ is the shielding constant for nucleus $x$ in conformer $i, R$ is the molar gas constant $(8.3145 \mathrm{~J}$ $\left.\mathrm{K}^{-1} \mathrm{~mol}^{-1}\right), T$ is the temperature $(298 \mathrm{~K})$, and $E_{i}$ is the SCF energy of conformer $i$ (relative to the lowest energy conformer), obtained at the corresponding level of theory (B3LYP/6-31G**//MMFF or $\left.\mathrm{PCM} / \mathrm{mPW} 1 \mathrm{PW} 91 / 6-31+\mathrm{G}^{* *} / / \mathrm{B} 3 \mathrm{LYP} / 6-31 \mathrm{G}^{*}\right)$. The scaled chemical shifts $\left(\delta_{s}\right)$ were computed as $\delta_{s}=$ $\left(\delta_{u}-b\right) / m$, where $m$ and $b$ are the slope and intercept, respectively, resulting from a linear regression calculation on a plot of $\delta_{u}$ against $\delta_{\text {exp }}$.

In the case of DP4 and DP4+ fitting procedures, the B3LYP/6-31G**//MMMF and $\mathrm{PCM} / \mathrm{mPW} 1 \mathrm{PW} 91 / 6-31+\mathrm{G}^{* *} / / \mathrm{B} 3 \mathrm{LYP} / 6-31 \mathrm{G}^{*}$ shielding constants, respectively, computed for all significant conformations of $\mathbf{1 - 8}$ were transformed into scaled chemical shifts using the experimental NMR data of ND or SD. Next, the corresponding $w_{\mathrm{i}}$ coefficients (conformational amplitudes) were optimized to minimize the CMAE (corrected mean absolute error) of each isomer with the boundaries conditions that $w_{\mathrm{i}} \geq 0$ and $\Sigma w_{\mathrm{i}}=1$ using the Solver module implemented in Excel. Once the $w_{\mathrm{i}}$ coefficients 
were optimized, the resulting averaged shielding values were computed, and the same procedure employed for the Boltzmann-averaged shelding values were carried out to compute DP4 and DP4+ probabilities.

The DP4, J-DP4 and DP4+ and calculations were carried out using the Excel spreadsheets available for free at sarotti-nmr.weebly.com, ${ }^{4,5}$ and the DIP calculations were carried out as indicated in the original publication. $^{7}$

For Random DP4+ calculations, the isotropic shielding values computed at the DP4+ level (PCM/mPW1PW91/6-31+G**//B3LYP/6-31G*) for each isomer were weighted using random conformational amplitudes created as indicated in the original paper. ${ }^{8}$ After 100.000 iterations using a home-made Matlab script, the resulting DP4+ values were averaged.

The ECD calculations were carried out using the B3LYP/6-31G* optimized geometries employed for DP4+ calculations. The excitation energies $(\mathrm{nm})$ and rotatory strenght $(R)$ in dipole velocity $\left(R_{\mathrm{vel}}\right)$ of the first twenty singlet excitations were calculated using TDDFT implemented in Gaussian 09 at the PCM/B3LYP/6-31G* level of theory (solvent=trifluoroethanol) from all significanlty pouplated conformers, which were averaged using Boltzmann weighting. The calculated rotatory strenghts were simulated into the ECD curve as the sum of Gaussians with $0.5 \mathrm{eV}$ width at half-heights $(\sigma)$.

\section{References:}

1) Frisch, M. J.; Trucks, G. W.; Schlegel, H. B.; Scuseria, G. E.; Robb, M. A.; Cheeseman, J. R.; Scalmani, G.; Barone, V.; Mennucci, B.; Petersson, G. A.; Nakatsuji, H.; Caricato, M.; Li, X.; Hratchian, H. P.; Izmaylov, A. F.; Bloino, J.; Zheng, G.; Sonnenberg, J. L.; Hada, M.; Ehara, M.; Toyota, K.; Fukuda, R.; Hasegawa, J.; Ishida, M.; Nakajima, T.; Honda, Y.; Kitao, O.; Nakai, H.; Vreven, T.; Montgomery, J. A., Jr., Peralta, J. E.; Ogliaro, F.; Bearpark, M.; Heyd, J. J.; Brothers, E.; Kudin, K. N.; Staroverov, V. N.; Kobayashi, R.; Normand, J.; Raghavachari, K.; Rendell, A.; Burant, J. C.; Iyengar, S. S.; Tomasi, J.; Cossi, M.; Rega, N.; Millam, J. M.; Klene, M.; Knox, J. E.; Cross, J. B.; Bakken, V.; Adamo, C.; Jaramillo, J.; Gomperts, R.; Stratmann, R. E.; Yazyev, O.; Austin, A. J.; Cammi, R.; Pomelli, C.; Ochterski, J. W.; Martin, R. L.; Morokuma, K.; Zakrzewski, V. G.; Voth, G. A.; Salvador, P.; Dannenberg, J. J.; Dapprich, S.; Daniels, A. D.; Farkas, O.; Foresman, J. B.; Ortiz, J. V.; Cioslowski, J.; Fox, D. J. Gaussian 09, Gaussian, Inc.: Wallingford, CT, 2009.

2) MacroModel Schrodinger release 2018-3; Schrodinger LLC: New York, 2018.

3) Smith, S. G.; Goodman, J. M. Assigning Stereochemistry to Single Diastereoisomers by GIAO NMR Calculation: The DP4 Probability. J. Am. Chem. Soc. 2010, 132, 12946.

4) Grimblat, N.; Gavin, J. A.; Hernández Daranas, A.; Sarotti, A. M. Combining the Power of $J$ Coupling and DP4 Analysis on Stereochemical Assignments: The J-DP4 Methods. Org. Lett. 2019, 21, 4003.

5) Grimblat, N.; Zanardi, M. M.; Sarotti, A. M. Beyond DP4: an Improved Probability for the Stereochemical Assignment of Isomeric Compounds using Quantum Chemical Calculations of NMR Shifts. J. Org. Chem. 2015, 80, 12526.

6) Tomasi, J.; Mennucci, B.; Cammi, R. Quantum Mechanical Continuum Solvation Models. Chem. Rev.2005, 105, 2999.

7) Zanardi, M. M.; Biglione, F. A.; Sortino, M. A.; Sarotti, A. M. General Quantum-Based NMR Method for the Assignment of Absolute Configuration by Single or Double Derivatization: Scope and Limitations. J. Org. Chem. 2018, 83, 11839.

8) Zanardi, M. M.; Marcarino, M. O.; Sarotti, A. M. Redefining the Impact of Boltzmann Analysis in the Stereochemical Assignment of Polar and Flexible Molecules by NMR Calculations. Org. Lett. 2020, 22, 52. 


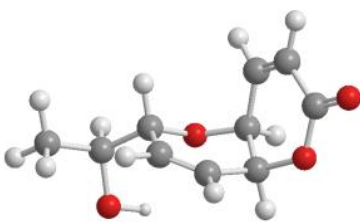

1-c01

$E_{\text {rel }}=0.00 \mathrm{kcal} / \mathrm{mol}$

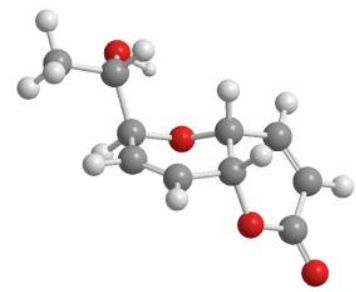

2-c17

$E_{\text {rel }}=0.00 \mathrm{kcal} / \mathrm{mol}$

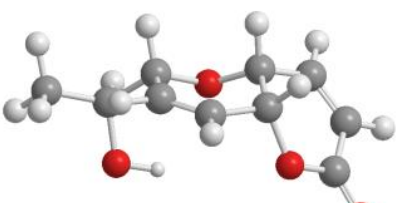

3-c01

$E_{\text {rel }}=0.00 \mathrm{kcal} / \mathrm{mol}$

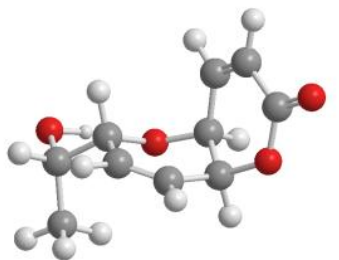

1-c04

$E_{\text {rel }}=0.20 \mathrm{kcal} / \mathrm{mol}$

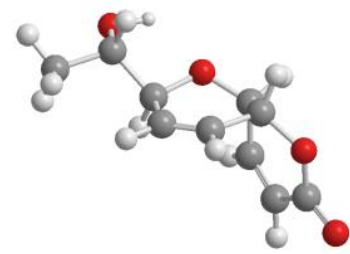

2-c05

$\mathrm{E}_{\mathrm{rel}}=0.46 \mathrm{kcal} / \mathrm{mol}$

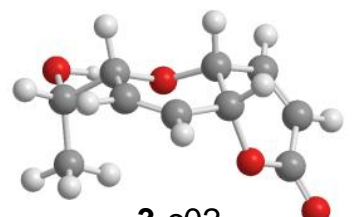

3-c02

$\mathrm{E}_{\text {rel }}=0.16 \mathrm{kcal} / \mathrm{mol}$

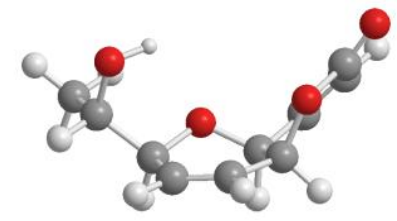

4-c01

$\mathrm{E}_{\mathrm{rel}}=0.61 \mathrm{kcal} / \mathrm{mol}$

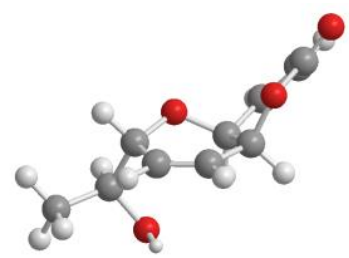

1-c03

$E_{\text {rel }}=0.76 \mathrm{kcal} / \mathrm{mol}$

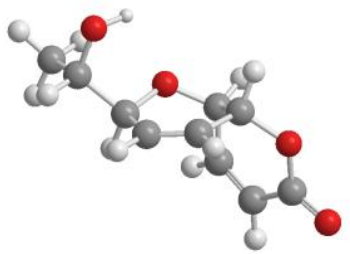

2-c02

$\mathrm{E}_{\mathrm{rel}}=1.19 \mathrm{kcal} / \mathrm{mol}$
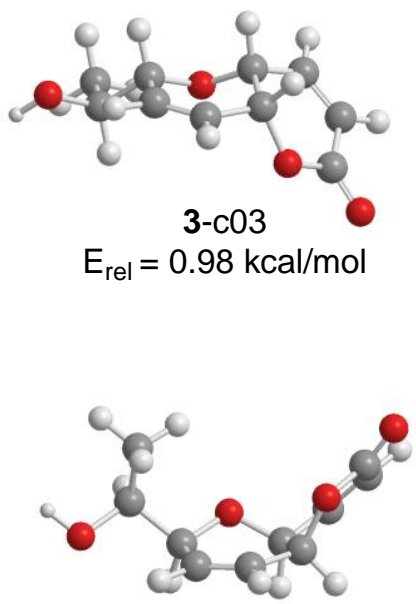

4-c03

$E_{\text {rel }}=1.52 \mathrm{kcal} / \mathrm{mol}$

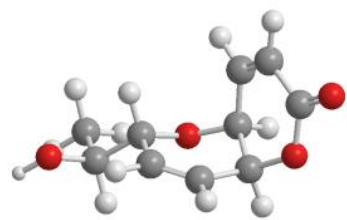

1-c09

$\mathrm{E}_{\mathrm{rel}}=1.09 \mathrm{kcal} / \mathrm{mol}$

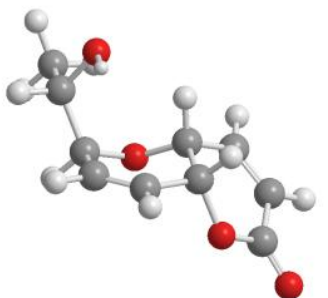

2-c04

$\mathrm{E}_{\mathrm{rel}}=1.40 \mathrm{kcal} / \mathrm{mol}$

$E_{\text {rel }}=0.00 \mathrm{kcal} / \mathrm{mol}$

Figure S1. Most stable conformations found for compounds 1-4 at the PCM/mPW1PW91/6-31+G**//B3LYP/6-31G* level of theory. 


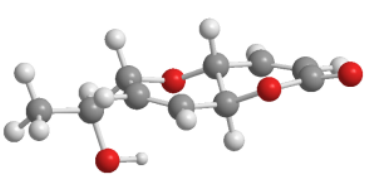

5-c01

$\mathrm{E}_{\text {rel }}=0.00 \mathrm{kcal} / \mathrm{mol}$

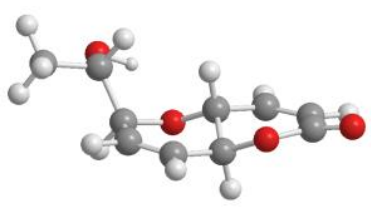

6-c01

$\mathrm{E}_{\mathrm{rel}}=0.00 \mathrm{kcal} / \mathrm{mol}$

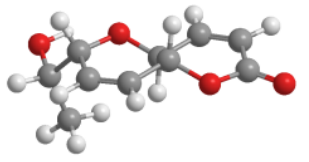

7-c06

$\mathrm{E}_{\mathrm{rel}}=0.00 \mathrm{kcal} / \mathrm{mol}$

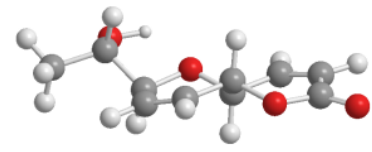

8-c02

$\mathrm{E}_{\mathrm{rel}}=0.00 \mathrm{kcal} / \mathrm{mol}$

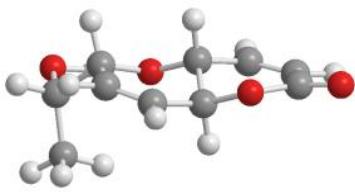

5- 02

$\mathrm{E}_{\mathrm{rel}}=0.27 \mathrm{kcal} / \mathrm{mol}$

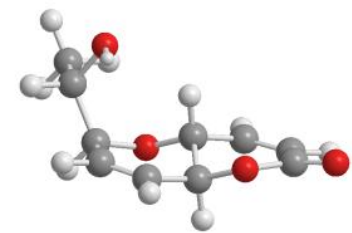

6-c02

$\mathrm{E}_{\mathrm{rel}}=1.37 \mathrm{kcal} / \mathrm{mol}$

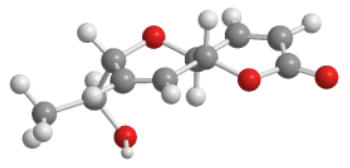

7-c02

$\mathrm{E}_{\mathrm{rel}}=0.04 \mathrm{kcal} / \mathrm{mol}$

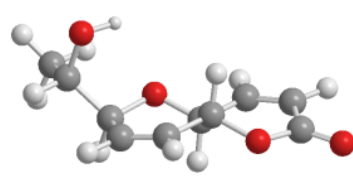

8-c01

$\mathrm{E}_{\mathrm{rel}}=0.61 \mathrm{kcal} / \mathrm{mol}$

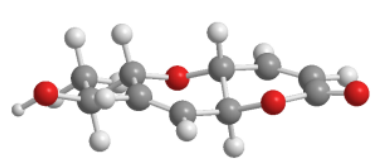

5-c03

$\mathrm{E}_{\mathrm{rel}}=1.10 \mathrm{kcal} / \mathrm{mol}$

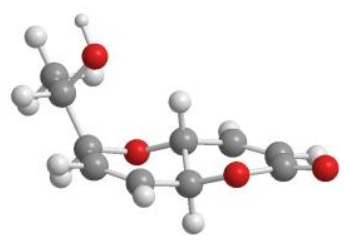

6- $\mathrm{C03}$

$\mathrm{E}_{\mathrm{rel}}=1.80 \mathrm{kcal} / \mathrm{mol}$

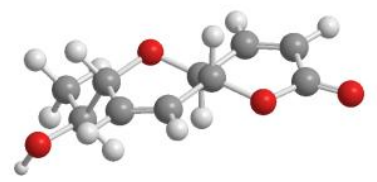

7-c01

$\mathrm{E}_{\text {rel }}=0.16 \mathrm{kcal} / \mathrm{mol}$

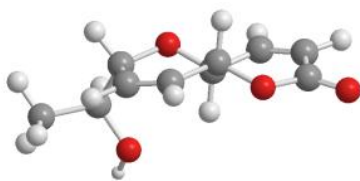

7- $\mathrm{c} 03$

$E_{\text {rel }}=0.39 \mathrm{kcal} / \mathrm{mol}$

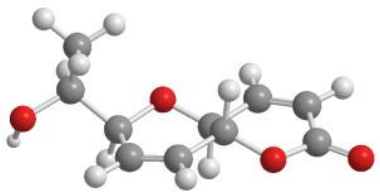

8- $\mathrm{C} 04$

$\mathrm{E}_{\mathrm{rel}}=1.59 \mathrm{kcal} / \mathrm{mol}$

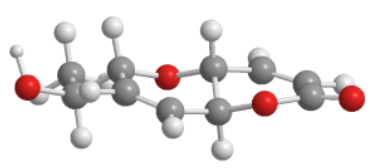

5-c04

$\mathrm{E}_{\mathrm{rel}}=1.14 \mathrm{kcal} / \mathrm{mol}$

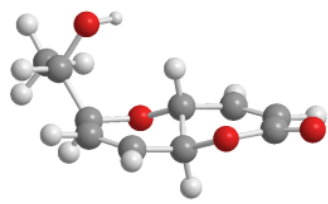

6-c04

$E_{\text {rel }}=2.45 \mathrm{kcal} / \mathrm{mol}$

Figure S2. Most stable conformations found for compounds 5-8 at the PCM/mPW1PW91/6-31+G**//B3LYP/6-31G* level of theory. 


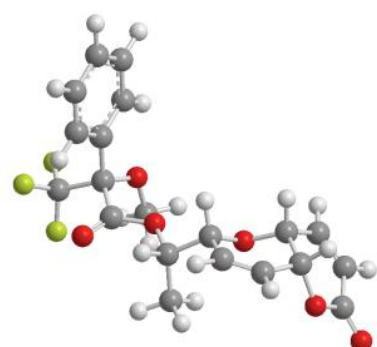

(R)-MPTA-3-c053

$\mathrm{E}_{\mathrm{rel}}=0.00 \mathrm{kcal} / \mathrm{mol}$

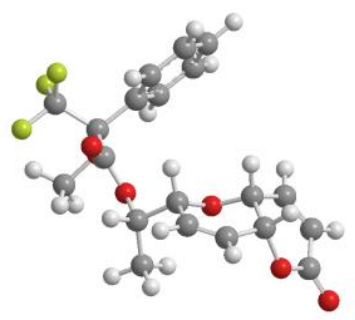

(R)-MPTA-3-c017

$\mathrm{E}_{\mathrm{rel}}=0.01 \mathrm{kcal} / \mathrm{mol}$

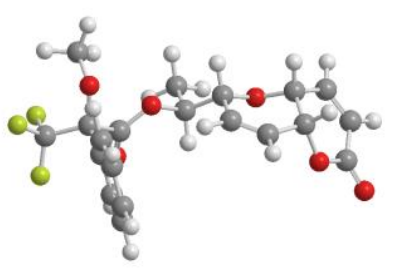

(R)-MPTA-3-c042

$\mathrm{E}_{\mathrm{rel}}=0.19 \mathrm{kcal} / \mathrm{mol}$

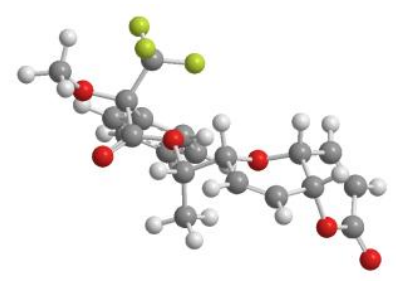

(R)-MPTA-3-c043

$\mathrm{E}_{\text {rel }}=0.76 \mathrm{kcal} / \mathrm{mol}$

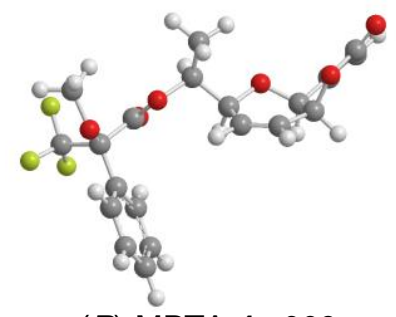

(R)-MPTA-4-c009

$\mathrm{E}_{\mathrm{rel}}=0.00 \mathrm{kcal} / \mathrm{mol}$

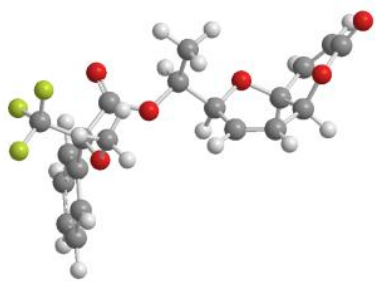

(R)-MPTA-4-c021

$\mathrm{E}_{\mathrm{rel}}=0.07 \mathrm{kcal} / \mathrm{mol}$

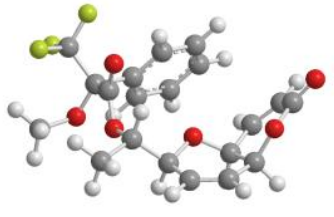

(R)-MPTA-4-c044

$\mathrm{E}_{\mathrm{rel}}=0.97 \mathrm{kcal} / \mathrm{mol}$

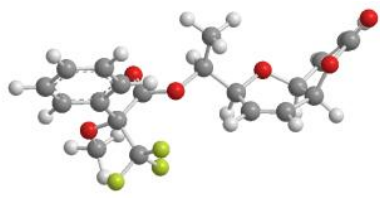

(R)-MPTA-4-c098

$\mathrm{E}_{\mathrm{rel}}=1.04 \mathrm{kcal} / \mathrm{mol}$

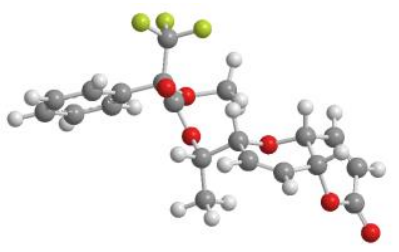

(S)-MPTA-3-c071

$\mathrm{E}_{\mathrm{rel}}=0.00 \mathrm{kcal} / \mathrm{mol}$

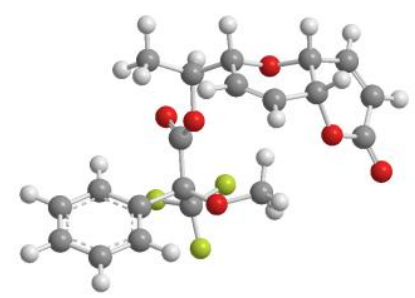

(S)-MPTA-3-c015

$\mathrm{E}_{\mathrm{rel}}=0.07 \mathrm{kcal} / \mathrm{mol}$

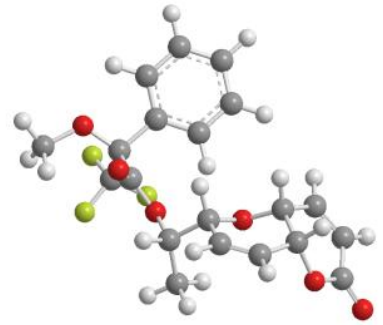

(S)-MPTA-3-c093

$E_{\text {rel }}=0.16 \mathrm{kcal} / \mathrm{mol}$

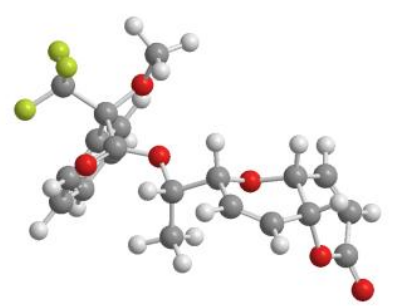

(S)-MPTA-3-c058

$\mathrm{E}_{\mathrm{rel}}=0.28 \mathrm{kcal} / \mathrm{mol}$

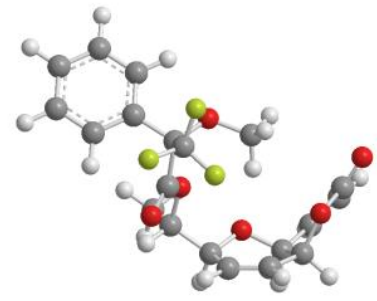

(S)-MPTA-4-c011

$\mathrm{E}_{\mathrm{rel}}=0.00 \mathrm{kcal} / \mathrm{mol}$

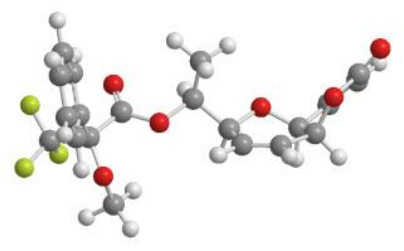

(S)-MPTA-4-c024

$\mathrm{E}_{\mathrm{rel}}=0.02 \mathrm{kcal} / \mathrm{mol}$

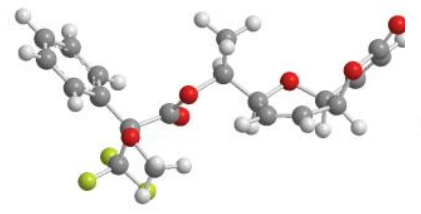

(S)-MPTA-4-c016

$\mathrm{E}_{\text {rel }}=0.27 \mathrm{kcal} / \mathrm{mol}$

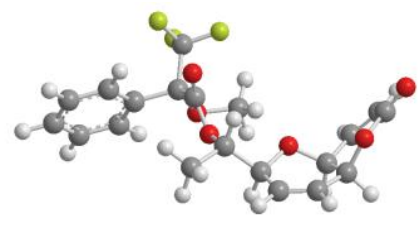

(S)-MPTA-4-c046

$\mathrm{E}_{\text {rel }}=0.27 \mathrm{kcal} / \mathrm{mol}$

Figure S3. Most stable conformations found for compounds $(R)$-MPTA-3, $(R)$-MPTA-4, $(S)$-MPTA-3 and $(S)$-MPTA-4 at the PCM/mPW1PW91/6-31+G**//B3LYP/6-31G* level of theory. 
Table S1: Boltzmann-averaged isotropic shielding values computed for compounds 1-8 at the B3LYP/6-31G**//MMFF level of theory.

\begin{tabular}{|c|c|c|c|c|c|c|c|c|}
\hline Atom & Isomer 1 & Isomer 2 & Isomer 3 & Isomer 4 & Isomer 5 & Isomer 6 & Isomer 7 & Isomer 8 \\
\hline C-2 & 36.1773 & 35.4381 & 35.0800 & 35.1342 & 33.5088 & 33.4545 & 33.4022 & 33.5525 \\
\hline C-3 & 69.6402 & 68.5592 & 67.9636 & 67.8302 & 71.3785 & 71.7988 & 72.4824 & 71.3649 \\
\hline C-4 & 53.2325 & 55.7614 & 57.3064 & 57.5190 & 49.0109 & 48.5343 & 47.9132 & 49.0953 \\
\hline $\mathrm{C}-4 \mathrm{a}$ & 125.6851 & 128.6848 & 124.7253 & 124.8030 & 119.0091 & 123.9787 & 122.7027 & 119.1124 \\
\hline C-6 & 115.5552 & 112.3031 & 110.9066 & 109.6684 & 108.4139 & 109.4302 & 111.6954 & 106.9890 \\
\hline C-7 & 64.6806 & 62.9031 & 60.3371 & 60.3552 & 64.9042 & 66.4296 & 67.9320 & 65.1589 \\
\hline $\mathrm{C}-8$ & 65.9797 & 67.5846 & 68.6432 & 68.4957 & 64.1933 & 62.8354 & 61.1909 & 64.0470 \\
\hline $\mathrm{C}-8 \mathrm{a}$ & 121.8113 & 122.3356 & 121.5389 & 121.5123 & 116.4512 & 116.6573 & 116.6963 & 116.6356 \\
\hline C-9 & 119.5698 & 122.8881 & 120.8070 & 120.8521 & 120.8375 & 122.7451 & 118.9291 & 120.6876 \\
\hline $\mathrm{C}-10$ & 171.9042 & 173.5118 & 172.9085 & 173.0579 & 172.8587 & 173.3931 & 170.0728 & 172.9872 \\
\hline $\mathrm{H}-3$ & 25.8030 & 25.6679 & 25.6051 & 25.5979 & 25.8241 & 25.8464 & 25.8930 & 25.8196 \\
\hline $\mathrm{H}-4$ & 25.0131 & 25.0642 & 25.0719 & 25.0687 & 24.8809 & 24.9073 & 24.8697 & 24.8804 \\
\hline $\mathrm{H}-4 \mathrm{a}$ & 26.7932 & 27.2577 & 27.9451 & 27.9476 & 27.3421 & 27.0372 & 26.7544 & 27.3591 \\
\hline H-6 & 27.6295 & 27.8150 & 27.7458 & 27.9594 & 27.4845 & 27.8129 & 27.6205 & 27.6950 \\
\hline $\mathrm{H}-7$ & 25.5869 & 25.5031 & 25.3722 & 25.4465 & 25.7345 & 25.7919 & 25.7164 & 25.7975 \\
\hline $\mathrm{H}-8$ & 25.4030 & 25.4276 & 25.4184 & 25.4506 & 25.4059 & 25.3521 & 25.2125 & 25.4320 \\
\hline $\mathrm{H}-8 \mathrm{a}$ & 26.8570 & 27.2103 & 27.3076 & 27.3030 & 26.8865 & 26.9705 & 27.0258 & 26.8958 \\
\hline H-9 & 27.7566 & 27.6830 & 27.7286 & 27.7210 & 27.7956 & 27.5721 & 27.6595 & 27.8101 \\
\hline H-10 & 30.6665 & 30.6252 & 30.6107 & 30.6220 & 30.6351 & 30.6029 & 30.6345 & 30.6268 \\
\hline
\end{tabular}

Table S2: Experimental chemical shifts reported for synthetic diplopyrone, and scaled chemical shifts computed for compounds 1-8 at the B3LYP/6-31G**//MMFF level of theory.

\begin{tabular}{|c|c|c|c|c|c|c|c|c|c|}
\hline Atom & $\operatorname{Exp}$ & Isomer 1 & Isomer 2 & Isomer 3 & Isomer 4 & Isomer 5 & Isomer 6 & Isomer 7 & Isomer 8 \\
\hline C-2 & 161.7 & 159.7 & 160.2 & 160.6 & 160.5 & 160.3 & 160.2 & 160.8 & 160.2 \\
\hline $\mathrm{C}-3$ & 123.3 & 124.3 & 125.7 & 125.8 & 125.8 & 120.1 & 120.2 & 119.2 & 120.0 \\
\hline C-4 & 144.2 & 141.7 & 139.1 & 137.0 & 136.8 & 143.8 & 144.4 & 145.4 & 143.7 \\
\hline $\mathrm{C}-4 \mathrm{a}$ & 64.9 & 65.1 & 63.0 & 65.6 & 65.4 & 69.6 & 65.8 & 65.8 & 69.3 \\
\hline C-6 & 74.8 & 75.8 & 80.1 & 80.3 & 81.5 & 80.8 & 80.9 & 77.5 & 82.2 \\
\hline C-7 & 129.6 & 129.6 & 131.6 & 133.8 & 133.8 & 127.0 & 125.8 & 124.1 & 126.6 \\
\hline C-8 & 124.7 & 128.2 & 126.7 & 125.0 & 125.1 & 127.7 & 129.5 & 131.3 & 127.8 \\
\hline C-8a & 70.0 & 69.2 & 69.7 & 69.0 & 68.9 & 72.3 & 73.4 & 72.2 & 71.9 \\
\hline C-9 & 69.7 & 71.6 & 69.1 & 69.8 & 69.6 & 67.6 & 67.1 & 69.8 & 67.6 \\
\hline C-10 & 18.7 & 16.3 & 16.3 & 14.6 & 14.2 & 12.5 & 14.3 & 15.4 & 12.1 \\
\hline $\mathrm{H}-3$ & 6.07 & 5.92 & 6.13 & 6.24 & 6.28 & 5.96 & 5.93 & 5.82 & 6.00 \\
\hline $\mathrm{H}-4$ & 6.88 & 6.69 & 6.72 & 6.76 & 6.79 & 6.88 & 6.86 & 6.82 & 6.91 \\
\hline $\mathrm{H}-4 \mathrm{a}$ & 4.92 & 4.95 & 4.58 & 3.98 & 4.01 & 4.47 & 4.76 & 4.98 & 4.49 \\
\hline H-6 & 4.08 & 4.14 & 4.04 & 4.17 & 4.00 & 4.33 & 3.99 & 4.14 & 4.16 \\
\hline $\mathrm{H}-7$ & 6.06 & 6.13 & 6.29 & 6.47 & 6.42 & 6.05 & 5.99 & 5.99 & 6.02 \\
\hline H-8 & 6.04 & 6.31 & 6.36 & 6.42 & 6.42 & 6.37 & 6.42 & 6.48 & 6.37 \\
\hline $\mathrm{H}-8 \mathrm{a}$ & 4.97 & 4.89 & 4.63 & 4.60 & 4.63 & 4.92 & 4.82 & 4.71 & 4.94 \\
\hline $\mathrm{H}-9$ & 3.96 & 4.02 & 4.17 & 4.19 & 4.23 & 4.02 & 4.23 & 4.10 & 4.05 \\
\hline $\mathrm{H}-10$ & 1.25 & 1.18 & 1.30 & 1.40 & 1.44 & 1.24 & 1.24 & 1.20 & 1.29 \\
\hline
\end{tabular}

\begin{tabular}{|c|c|c|c|c|c|c|c|c|c|}
\hline \multicolumn{2}{|c|}{ Isomer № } & 1 & 2 & 3 & 4 & 5 & 6 & 7 & 8 \\
\hline \multirow{4}{*}{ DP4 (\%) } & $\mathrm{H}$ & 77.99 & 0.20 & 0.00 & 0.00 & 3.12 & 6.11 & 2.63 & 9.96 \\
\cline { 2 - 10 } & $\mathrm{C}$ & 99.20 & 0.70 & 0.04 & 0.01 & 0.00 & 0.01 & 0.04 & 0.00 \\
\cline { 2 - 10 } & $\mathrm{H}+\mathrm{C}$ & 100.00 & 0.00 & 0.00 & 0.00 & 0.00 & 0.00 & 0.00 & 0.00 \\
\cline { 2 - 10 } & $\mathrm{J}$ & - & - & - & - & - & - & - & - \\
\cline { 2 - 10 } & all data & 100.00 & 0.00 & 0.00 & 0.00 & 0.00 & 0.00 & 0.00 & 0.00 \\
\hline
\end{tabular}

Figure S4: DP4 results obtained by correlating the experimental NMR data reported for synthetic diplopyrone with the NMR data of compounds 1-8 calculated at the B3LYP/6-31G**//MMFF level of theory. 
Table S3: Experimental chemical shifts reported for natural diplopyrone, and scaled chemical shifts computed for compounds 1-8 at the B3LYP/6-31G**//MMFF level of theory.

\begin{tabular}{|c|c|c|c|c|c|c|c|c|c|}
\hline Atom & $\operatorname{Exp}$ & Isomer 1 & Isomer 2 & Isomer 3 & Isomer 4 & Isomer 5 & Isomer 6 & Isomer 7 & Isomer 8 \\
\hline C-2 & 162.7 & 159.9 & 160.3 & 160.6 & 160.4 & 160.4 & 160.4 & 161.1 & 160.4 \\
\hline $\mathrm{C}-3$ & 124.8 & 124.5 & 125.8 & 125.8 & 125.9 & 120.3 & 120.4 & 119.5 & 120.2 \\
\hline C-4 & 140.0 & 141.9 & 139.1 & 137.1 & 136.8 & 144.0 & 144.6 & 145.7 & 143.9 \\
\hline $\mathrm{C}-4 \mathrm{a}$ & 64.9 & 65.3 & 63.3 & 65.9 & 65.7 & 69.8 & 66.0 & 65.9 & 69.5 \\
\hline C-6 & 78.9 & 76.0 & 80.3 & 80.5 & 81.7 & 81.0 & 81.1 & 77.7 & 82.4 \\
\hline C-7 & 132.6 & 129.8 & 131.7 & 133.9 & 133.8 & 127.1 & 126.0 & 124.3 & 126.8 \\
\hline C-8 & 123.0 & 128.4 & 126.9 & 125.1 & 125.2 & 127.9 & 129.7 & 131.5 & 128.0 \\
\hline $\mathrm{C}-8 \mathrm{a}$ & 69.7 & 69.4 & 69.9 & 69.3 & 69.2 & 72.5 & 73.6 & 72.3 & 72.1 \\
\hline C-9 & 69.0 & 71.8 & 69.3 & 70.1 & 69.9 & 67.8 & 67.2 & 70.0 & 67.8 \\
\hline $\mathrm{C}-10$ & 17.8 & 16.5 & 16.7 & 15.1 & 14.7 & 12.7 & 14.4 & 15.4 & 12.3 \\
\hline H-3 & 6.23 & 5.89 & 6.09 & 6.17 & 6.21 & 5.91 & 5.90 & 5.78 & 5.95 \\
\hline $\mathrm{H}-4$ & 6.88 & 6.70 & 6.70 & 6.70 & 6.73 & 6.87 & 6.87 & 6.83 & 6.91 \\
\hline $\mathrm{H}-4 \mathrm{a}$ & 4.09 & 4.87 & 4.49 & 3.88 & 3.91 & 4.37 & 4.66 & 4.90 & 4.39 \\
\hline H-6 & 4.16 & 4.01 & 3.92 & 4.08 & 3.90 & 4.22 & 3.86 & 4.01 & 4.04 \\
\hline H-7 & 6.18 & 6.11 & 6.26 & 6.40 & 6.36 & 6.00 & 5.95 & 5.96 & 5.97 \\
\hline H-8 & 6.14 & 6.30 & 6.33 & 6.36 & 6.36 & 6.34 & 6.41 & 6.48 & 6.35 \\
\hline $\mathrm{H}-8 \mathrm{a}$ & 4.65 & 4.81 & 4.53 & 4.51 & 4.55 & 4.83 & 4.73 & 4.62 & 4.86 \\
\hline H-9 & 3.92 & 3.88 & 4.06 & 4.09 & 4.14 & 3.90 & 4.11 & 3.97 & 3.93 \\
\hline H-10 & 1.21 & 0.88 & 1.09 & 1.27 & 1.30 & 1.01 & 0.97 & 0.91 & 1.06 \\
\hline
\end{tabular}

\begin{tabular}{|c|c|c|c|c|c|c|c|c|c|c|}
\hline \multicolumn{2}{|c|}{ Isomer No } & 1 & 2 & 3 & 4 & 5 & 6 & 7 & 8 \\
\hline & $\mathrm{H}$ & 0.01 & 5.68 & 42.75 & 26.22 & 14.62 & 0.02 & 0.00 & 10.69 \\
\cline { 2 - 10 } & $\mathrm{C}$ & 0.93 & 68.96 & 22.33 & 7.78 & 0.00 & 0.00 & 0.00 & 0.00 \\
\cline { 2 - 10 } & $\mathrm{H}+\mathrm{C}$ & 0.00 & 25.25 & 61.59 & 13.16 & 0.00 & 0.00 & 0.00 & 0.00 \\
\cline { 2 - 10 } & $\mathrm{J}$ & - & - & - & - & - & - & 0.00 & 0.00 \\
\cline { 2 - 10 } & all data & 0.00 & 25.25 & 61.59 & 13.16 & 0.00 & 0.00 & 0.00
\end{tabular}

Figure S5: DP4 results obtained by correlating the experimental NMR data reported for natural diplopyrone with the NMR data of compounds 1-8 calculated at the B3LYP/6-31G**//MMFF level of theory.

Table S4: Bolzmtann-averaged isotropic shielding values computed for compounds 1-8 at the B3LYP/6-31G**//MMFF level of theory after removing conformations incompatible with the experimental ${ }^{3} J_{4 a-8 a}$ and ${ }^{3} J_{6-9}$ values.

\begin{tabular}{ccccccccc}
\hline Atom & Isomer 1 & Isomer 2 & Isomer 3 & Isomer 4 & Isomer 5 & Isomer 6 & Isomer 7 & Isomer 8 \\
\hline C-2 & 36.1373 & 35.3898 & 35.0918 & 35.1050 & - & - & - & - \\
C-3 & 69.6583 & 69.4741 & 67.9380 & 67.9122 & - & - & - & - \\
C-4 & 53.2839 & 55.0878 & 57.3240 & 57.4979 & - & - & - & - \\
C-4a & 125.7250 & 127.0528 & 124.7553 & 124.5415 & - & - & - & - \\
C-6 & 115.6336 & 115.0969 & 110.9714 & 111.7568 & - & - & - & - \\
C-7 & 64.9683 & 61.9340 & 60.5558 & 57.3168 & - & - & - & - \\
C-8 & 65.7621 & 65.5043 & 68.4662 & 68.7214 & - & - & - & - \\
C-8a & 121.8047 & 122.1900 & 121.5092 & 121.7424 & - & - & - & - \\
C-9 & 119.2991 & 119.5323 & 120.7613 & 120.4996 & - & - & - & - \\
C-10 & 172.2019 & 171.5508 & 173.3874 & 170.0738 & - & - & - & - \\
& & & & & & - & - \\
H-3 & 25.8004 & 25.7325 & 25.5999 & 25.6011 & - & - & - & - \\
H-4 & 25.0105 & 25.0179 & 25.0684 & 25.0744 & - & - & - & - \\
H-4a & 26.7638 & 26.7277 & 27.9370 & 27.9385 & - & - & - \\
H-6 & 27.5722 & 27.7452 & 27.6793 & 27.8282 & - & - & - & - \\
H-7 & 25.6158 & 25.5434 & 25.4127 & 25.3059 & - & - & - & - \\
H-8 & 25.3907 & 25.2824 & 25.4140 & 25.4018 & - & - & - & - \\
H-8a & 26.8660 & 27.1587 & 27.3027 & 27.3036 & - & - & - & - \\
H-9 & 27.7245 & 27.7606 & 27.7019 & 27.8010 & - & - & - \\
H-10 & 30.6748 & 30.4645 & 30.6197 & 30.5904 & - & - & - & - \\
\hline
\end{tabular}


Table S5: Experimental chemical shifts and ${ }^{3} J_{\mathrm{HH}}$ reported for synthetic diplopyrone, scaled chemical shifts and ${ }^{3} J$ computed for compounds 1-8 at the B3LYP/6-31G**//MMFF level of theory after removing conformations incompatible with the experimental ${ }^{3} J_{4 a-8 a}$ and ${ }^{3} J_{6-9}$ values.

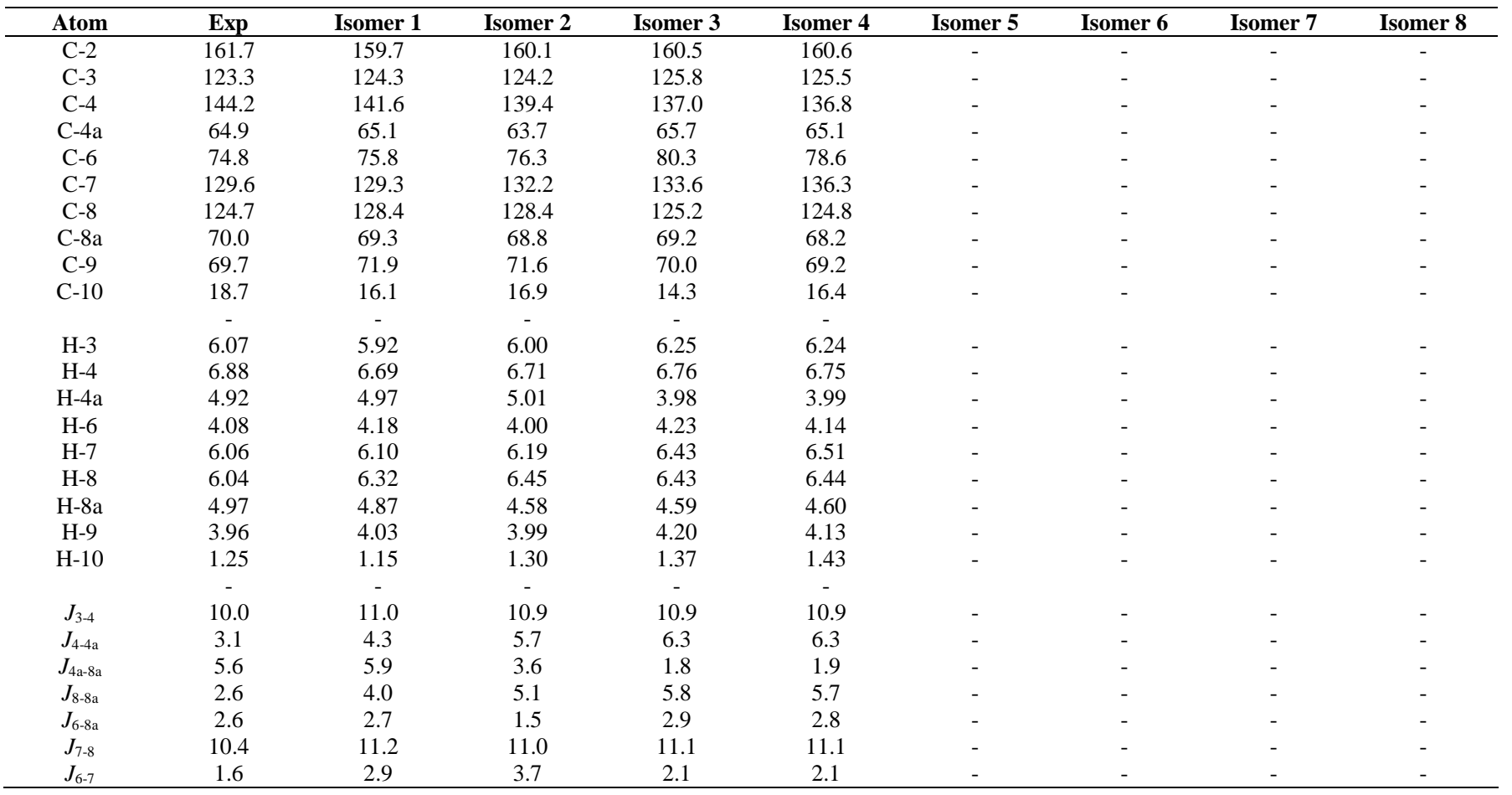

\begin{tabular}{|c|c|c|c|c|c|c|c|c|c|}
\hline \multicolumn{2}{|c|}{ Isomer № } & 1 & 2 & 3 & 4 & 5 & 6 & 7 & 8 \\
\hline \multirow{5}{*}{ DP4 (\%) } & $\mathrm{H}$ & 95.40 & 4.60 & 0.00 & 0.00 & - & - & - & - \\
\cline { 2 - 10 } & $\mathrm{C}$ & 93.17 & 6.69 & 0.05 & 0.08 & - & - & - & - \\
\cline { 2 - 10 } & $H+C$ & 99.65 & 0.35 & 0.00 & 0.00 & - & - & - & - \\
\cline { 2 - 10 } & $\mathrm{J}$ & 98.90 & 0.38 & 0.31 & 0.41 & - & - & - & - \\
\cline { 2 - 9 } & all data & 100.00 & 0.00 & 0.00 & 0.00 & - & - & - & - \\
\hline
\end{tabular}

Figure S6: $J$-DP4 results obtained by correlating the experimental NMR data reported for synthetic diplopyrone with the NMR data of compounds 1-8 calculated at the B3LYP/6-31G**//MMFF level of theory after removing conformations incompatible with the experimental ${ }^{3} J_{4 a-8 a}$ and ${ }^{3} J_{6-9}$ values. 
Table S6: Experimental chemical shifts and ${ }^{3} J_{\mathrm{HH}}$ reported for natural diplopyrone, scaled chemical shifts and ${ }^{3} J$ computed for compounds 1-8 at the B3LYP/6-31G**//MMFF level of theory after removing conformations incompatible with the experimental ${ }^{3} J_{4 a-8 a}$ and ${ }^{3} J_{6-9}$ values.

\begin{tabular}{|c|c|c|c|c|c|c|c|c|c|}
\hline Atom & Exp & Isomer 1 & Isomer 2 & Isomer 3 & Isomer 4 & Isomer 5 & Isomer 6 & Isomer 7 & Isomer 8 \\
\hline C-2 & 162.7 & 159.9 & 160.2 & 160.5 & 160.5 & - & - & - & - \\
\hline C-3 & 124.8 & 124.5 & 124.4 & 125.9 & 125.6 & - & - & - & - \\
\hline C-4 & 140.0 & 141.8 & 139.5 & 137.1 & 136.6 & - & - & - & - \\
\hline C-4a & 64.9 & 65.3 & 63.9 & 66.0 & 65.3 & - & - & - & - \\
\hline C-6 & 78.9 & 76.0 & 76.5 & 80.5 & 78.9 & - & - & - & - \\
\hline C-7 & 132.6 & 129.5 & 132.3 & 133.7 & 136.8 & - & - & - & - \\
\hline C-8 & 123.0 & 128.6 & 128.6 & 125.3 & 124.7 & - & - & - & - \\
\hline C-8a & 69.7 & 69.5 & 69.0 & 69.4 & 68.3 & - & - & - & - \\
\hline C-9 & 69.0 & 72.1 & 71.8 & 70.2 & 69.6 & - & - & - & - \\
\hline C-10 & 17.8 & 16.3 & 17.2 & 14.8 & 16.9 & - & - & - & - \\
\hline H-3 & 6.23 & 5.89 & 5.97 & 6.18 & 6.17 & - & - & - & - \\
\hline H-4 & 6.88 & 6.70 & 6.72 & 6.70 & 6.69 & - & - & - & - \\
\hline H-4a & 4.09 & 4.89 & 4.93 & 3.88 & 3.90 & - & - & - & - \\
\hline H-6 & 4.16 & 4.06 & 3.87 & 4.13 & 4.01 & - & - & - & - \\
\hline H-7 & 6.18 & 6.08 & 6.17 & 6.36 & 6.46 & - & - & - & - \\
\hline H-8 & 6.14 & 6.31 & 6.44 & 6.36 & 6.37 & - & - & - & - \\
\hline H-8a & 4.65 & 4.79 & 4.48 & 4.50 & 4.52 & - & - & - & - \\
\hline H-9 & 3.92 & 3.90 & 3.85 & 4.11 & 4.03 & - & - & - & - \\
\hline $\mathrm{H}-10$ & 1.21 & 0.85 & 1.03 & 1.24 & 1.31 & - & - & - & - \\
\hline$J_{3-4}$ & 9.8 & 11.0 & 10.9 & 10.9 & 10.9 & - & - & - & - \\
\hline$J_{4-4 a}$ & 5.8 & 4.3 & 5.7 & 6.3 & 6.3 & - & - & - & - \\
\hline$J_{4 \mathrm{a}-8 \mathrm{a}}$ & 2.8 & 5.9 & 3.6 & 1.8 & 1.9 & - & - & - & - \\
\hline$J_{8-8 \mathrm{a}}$ & 4.6 & 4.0 & 5.1 & 5.8 & 5.7 & - & - & - & - \\
\hline$J_{6-8 \mathrm{a}}$ & 3.5 & 2.7 & 1.5 & 2.9 & 2.8 & - & - & - & - \\
\hline$J_{7-8}$ & 10.4 & 11.2 & 11.0 & 11.1 & 11.1 & - & - & - & - \\
\hline$J_{6-7}$ & 0.0 & 2.9 & 3.7 & 2.1 & 2.1 & - & - & - & - \\
\hline
\end{tabular}

\begin{tabular}{|c|c|c|c|c|c|c|c|c|c|}
\hline \multicolumn{2}{|c|}{ Isomer No } & 1 & 2 & 3 & 4 & 5 & 6 & 7 & 8 \\
\hline & $\mathrm{H}$ & 0.01 & 0.01 & 79.81 & 20.17 & - & - & - & - \\
\cline { 2 - 10 } & $\mathrm{C}$ & 1.09 & 18.52 & 36.49 & 43.90 & - & - & - & - \\
\cline { 2 - 10 } \\
\cline { 2 - 10 } & $\mathrm{H}+\mathrm{C}$ & 0.00 & 0.00 & 76.68 & 23.31 & - & - & - & - \\
\cline { 2 - 10 } & $\mathrm{J}$ & 1.24 & 11.23 & 39.30 & 48.22 & - & - & - & - \\
\cline { 2 - 10 } & all data & 0.00 & 0.00 & 72.83 & 27.17 & - & - & - & - \\
\hline
\end{tabular}

Figure S7: $J$-DP4 results obtained by correlating the experimental NMR data reported for natural diplopyrone with the NMR data of compounds 1-8 calculated at the B3LYP/6-31G**//MMFF level of theory after removing conformations incompatible with the experimental ${ }^{3} J_{4 a-8 a}$ and ${ }^{3} J_{6-9}$ values. 
Table S7: Boltzmann-averaged isotropic shielding values computed for compounds 1-8 at the PCM/mPW1PW91/6$31+\mathrm{G}^{* *} / / \mathrm{B} 3 \mathrm{LYP} / 6-31 \mathrm{G}^{*}$ level of theory.

\begin{tabular}{|c|c|c|c|c|c|c|c|c|}
\hline Atom & Isomer 1 & Isomer 2 & Isomer 3 & Isomer 4 & Isomer 5 & Isomer 6 & Isomer 7 & Isomer 8 \\
\hline C-2 & 37.4344 & 37.1225 & 36.6027 & 36.7778 & 35.9471 & 36.0677 & 35.8696 & 36.0284 \\
\hline$C-3$ & 74.3739 & 73.2662 & 72.3198 & 72.1449 & 76.5209 & 76.5304 & 77.1955 & 76.4719 \\
\hline C-4 & 50.1374 & 52.8310 & 55.2084 & 55.5036 & 47.2227 & 47.2309 & 46.4642 & 47.4982 \\
\hline C- $-4 a$ & 128.3343 & 131.2781 & 129.3443 & 129.5615 & 122.7221 & 127.6464 & 126.0073 & 122.9416 \\
\hline C-6 & 119.4600 & 116.4460 & 115.7016 & 114.6313 & 113.9794 & 113.3613 & 116.1748 & 113.0391 \\
\hline C-7 & 66.8852 & 66.0530 & 63.3047 & 63.2341 & 68.6391 & 70.1042 & 69.7300 & 68.4551 \\
\hline C-8 & 71.7899 & 72.6657 & 73.7046 & 73.2829 & 69.8247 & 68.7175 & 68.7086 & 69.5490 \\
\hline C-8a & 124.1748 & 125.3267 & 124.9441 & 125.0236 & 119.9706 & 120.1154 & 119.9531 & 120.1995 \\
\hline C-9 & 124.0035 & 126.6311 & 124.8281 & 124.5776 & 124.6694 & 127.0212 & 123.4870 & 124.1877 \\
\hline C-10 & 176.8694 & 177.4388 & 177.6498 & 177.1731 & 177.7753 & 177.6401 & 174.8937 & 176.8725 \\
\hline $\mathrm{H}-3$ & 25.3850 & 25.2866 & 25.2071 & 25.1892 & 25.4323 & 25.4552 & 25.4913 & 25.4276 \\
\hline $\mathrm{H}-4$ & 24.2591 & 24.2938 & 24.2840 & 24.2750 & 24.1050 & 24.1644 & 24.1348 & 24.1019 \\
\hline $\mathrm{H}-4 \mathrm{a}$ & 26.5564 & 26.9228 & 27.4627 & 27.4432 & 27.1847 & 27.0934 & 26.9017 & 27.2091 \\
\hline H-6 & 27.3763 & 27.5740 & 27.2978 & 27.5149 & 27.1370 & 27.5181 & 27.2992 & 27.3496 \\
\hline $\mathrm{H}-7$ & 25.0435 & 25.0996 & 24.8937 & 25.0452 & 25.2952 & 25.4154 & 25.1662 & 25.4159 \\
\hline H-8 & 25.1990 & 25.1509 & 25.0561 & 25.0813 & 25.0876 & 25.0837 & 24.9980 & 25.0938 \\
\hline $\mathrm{H}-8 \mathrm{a}$ & 26.5992 & 26.8097 & 26.9302 & 26.9195 & 26.8130 & 26.8134 & 26.8632 & 26.8368 \\
\hline H-9 & 27.5986 & 27.6892 & 27.6257 & 27.8057 & 27.6129 & 27.5792 & 27.5181 & 27.8873 \\
\hline $\mathrm{H}-10$ & 30.3991 & 30.3817 & 30.3927 & 30.3573 & 30.4169 & 30.3833 & 30.3533 & 30.3725 \\
\hline
\end{tabular}

Table S8: Experimental chemical shifts reported for synthetic diplopyrone, and scaled chemical shifts computed for compounds 1-8 at the PCM/mPW1PW91/6-31+G**//B3LYP/6-31G* level of theory.

\begin{tabular}{|c|c|c|c|c|c|c|c|c|c|}
\hline Atom & $\operatorname{Exp}$ & Isomer 1 & Isomer 2 & Isomer 3 & Isomer 4 & Isomer 5 & Isomer 6 & Isomer 7 & Isomer 8 \\
\hline C-2 & 161.7 & 159.7 & 160.2 & 160.6 & 160.5 & 160.2 & 160.1 & 160.7 & 160.2 \\
\hline C-3 & 123.3 & 121.8 & 123.3 & 123.9 & 124.0 & 118.1 & 118.6 & 117.6 & 118.0 \\
\hline C-4 & 144.2 & 146.7 & 144.2 & 141.5 & 141.2 & 148.5 & 148.7 & 149.6 & 148.3 \\
\hline $\mathrm{C}-4 \mathrm{a}$ & 64.9 & 66.3 & 64.1 & 65.3 & 64.7 & 70.2 & 66.1 & 66.7 & 69.6 \\
\hline C-6 & 74.8 & 75.4 & 79.2 & 79.3 & 80.2 & 79.2 & 80.8 & 77.0 & 79.9 \\
\hline C-7 & 129.6 & 129.4 & 130.7 & 133.2 & 133.2 & 126.3 & 125.2 & 125.4 & 126.4 \\
\hline C-8 & 124.7 & 124.4 & 123.9 & 122.5 & 122.8 & 125.0 & 126.6 & 126.4 & 125.3 \\
\hline C-8a & 70.0 & 70.6 & 70.2 & 69.8 & 69.4 & 73.0 & 73.9 & 73.0 & 72.4 \\
\hline C-9 & 69.7 & 70.8 & 68.8 & 69.9 & 69.9 & 68.1 & 66.8 & 69.3 & 68.3 \\
\hline C-10 & 18.7 & 16.5 & 17.0 & 15.6 & 15.6 & 13.1 & 14.8 & 15.8 & 13.3 \\
\hline $\mathrm{H}-3$ & 6.07 & 5.93 & 6.09 & 6.15 & 6.21 & 5.95 & 5.98 & 5.87 & 6.02 \\
\hline $\mathrm{H}-4$ & 6.88 & 6.96 & 6.99 & 6.98 & 7.03 & 7.17 & 7.17 & 7.12 & 7.23 \\
\hline $\mathrm{H}-4 \mathrm{a}$ & 4.92 & 4.85 & 4.60 & 4.11 & 4.18 & 4.35 & 4.47 & 4.57 & 4.39 \\
\hline H-6 & 4.08 & 4.10 & 4.01 & 4.26 & 4.11 & 4.39 & 4.08 & 4.20 & 4.26 \\
\hline H-7 & 6.06 & 6.24 & 6.26 & 6.43 & 6.34 & 6.08 & 6.02 & 6.17 & 6.03 \\
\hline H-8 & 6.04 & 6.10 & 6.21 & 6.28 & 6.30 & 6.27 & 6.32 & 6.32 & 6.32 \\
\hline $\mathrm{H}-8 \mathrm{a}$ & 4.97 & 4.81 & 4.70 & 4.59 & 4.65 & 4.69 & 4.73 & 4.60 & 4.73 \\
\hline H-9 & 3.96 & 3.90 & 3.90 & 3.96 & 3.85 & 3.95 & 4.02 & 4.00 & 3.77 \\
\hline $\mathrm{H}-10$ & 1.25 & 1.33 & 1.46 & 1.47 & 1.56 & 1.38 & 1.43 & 1.39 & 1.50 \\
\hline
\end{tabular}

\begin{tabular}{|c|c|c|c|c|c|c|c|c|}
\hline & Isomer 1 & Isomer 2 & Isomer 3 & Isomer 4 & Isomer 5 & Isomer 6 & Isomer 7 & Isomer 8 \\
\hline sDP4+ (H data) & $99.46 \%$ & $0.44 \%$ & $0.00 \%$ & $0.00 \%$ & $0.01 \%$ & $0.08 \%$ & $0.01 \%$ & $0.00 \%$ \\
\hline sDP4+ (C data) & $40.50 \%$ & $59.28 \%$ & $0.16 \%$ & $0.06 \%$ & $0.00 \%$ & $0.00 \%$ & $0.00 \%$ & $0.00 \%$ \\
\hline sDP4+ (all data) & (매 $99.36 \%$ & $0.64 \%$ & $0.00 \%$ & $0.00 \%$ & $0.00 \%$ & $0.00 \%$ & $0.00 \%$ & $0.00 \%$ \\
\hline uDP4+ (H data) & $77.02 \%$ & $22.56 \%$ & $0.03 \%$ & $0.27 \%$ & $0.00 \%$ & $0.06 \%$ & $0.06 \%$ & $0.00 \%$ \\
\hline uDP4+ (C data) & (口) $98.57 \%$ & $1.00 \%$ & $0.30 \%$ & $0.13 \%$ & $0.00 \%$ & $0.00 \%$ & $0.00 \%$ & $0.00 \%$ \\
\hline uDP4+ (all data) & $99.70 \%$ & $0.30 \%$ & $0.00 \%$ & $0.00 \%$ & $0.00 \%$ & $0.00 \%$ & $0.00 \%$ & $0.00 \%$ \\
\hline DP4+ (H data) & 99.87\% & $0.13 \%$ & $0.00 \%$ & $0.00 \%$ & $0.00 \%$ & $0.00 \%$ & $0.00 \%$ & $0.00 \%$ \\
\hline DP4+ (C data) & \begin{tabular}{|l|l|l} 
& $98.53 \%$ \\
\end{tabular} & $1.47 \%$ & $0.00 \%$ & $0.00 \%$ & $0.00 \%$ & $0.00 \%$ & $0.00 \%$ & $0.00 \%$ \\
\hline DP4+ (all data) & $100.00 \%$ & $0.00 \%$ & $0.00 \%$ & $0.00 \%$ & $0.00 \%$ & $0.00 \%$ & $0.00 \%$ & $0.00 \%$ \\
\hline
\end{tabular}

Figure S8: DP4+ results obtained by correlating the experimental NMR data reported for synthetic diplopyrone with the NMR data of compounds 1-8 calculated at the PCM/mPW1PW91/6-31+G**//B3LYP/6-31G* level of theory. 
Table S9: Experimental chemical shifts reported for natural diplopyrone, and scaled chemical shifts computed for compounds 1-8 at the PCM/mPW1PW91/6-31+G**//B3LYP/6-31G* level of theory.

\begin{tabular}{|c|c|c|c|c|c|c|c|c|c|}
\hline Atom & Exp & Isomer 1 & Isomer 2 & Isomer 3 & Isomer 4 & Isomer 5 & Isomer 6 & Isomer 7 & Isomer 8 \\
\hline C-2 & 162.7 & 160.0 & 160.4 & 160.7 & 160.5 & 160.4 & 160.4 & 161.0 & 160.5 \\
\hline C-3 & 124.8 & 122.0 & 123.5 & 124.0 & 124.1 & 118.3 & 118.8 & 117.8 & 118.2 \\
\hline C-4 & 140.0 & 146.9 & 144.3 & 141.6 & 141.3 & 148.7 & 148.9 & 150.0 & 148.5 \\
\hline$C-4 a$ & 64.9 & 66.5 & 64.3 & 65.5 & 65.0 & 70.3 & 66.3 & 66.8 & 69.7 \\
\hline C-6 & 78.9 & 75.6 & 79.4 & 79.5 & 80.4 & 79.4 & 81.0 & 77.1 & 80.1 \\
\hline C-7 & 132.6 & 129.7 & 130.8 & 133.3 & 133.3 & 126.5 & 125.4 & 125.6 & 126.6 \\
\hline C-8 & 123.0 & 124.6 & 124.1 & 122.6 & 123.0 & 125.2 & 126.8 & 126.7 & 125.5 \\
\hline C-8a & 69.7 & 70.8 & 70.4 & 70.0 & 69.7 & 73.2 & 74.0 & 73.2 & 72.6 \\
\hline C-9 & 69.0 & 70.9 & 69.0 & 70.2 & 70.1 & 68.3 & 66.9 & 69.5 & 68.4 \\
\hline $\mathrm{C}-10$ & 17.8 & 16.6 & 17.2 & 16.0 & 16.0 & 13.1 & 14.9 & 15.7 & 13.4 \\
\hline $\mathrm{H}-3$ & 6.23 & 5.89 & 6.05 & 6.08 & 6.15 & 5.90 & 5.94 & 5.82 & 5.97 \\
\hline $\mathrm{H}-4$ & 6.88 & 6.98 & 6.99 & 6.93 & 6.99 & 7.16 & 7.17 & 7.11 & 7.22 \\
\hline $\mathrm{H}-4 \mathrm{a}$ & 4.09 & 4.77 & 4.50 & 4.01 & 4.08 & 4.24 & 4.37 & 4.47 & 4.29 \\
\hline H-6 & 4.16 & 3.98 & 3.89 & 4.16 & 4.01 & 4.29 & 3.96 & 4.09 & 4.15 \\
\hline $\mathrm{H}-7$ & 6.18 & 6.22 & 6.23 & 6.37 & 6.28 & 6.03 & 5.97 & 6.13 & 5.98 \\
\hline H-8 & 6.14 & 6.07 & 6.18 & 6.22 & 6.25 & 6.23 & 6.29 & 6.29 & 6.28 \\
\hline $\mathrm{H}-8 \mathrm{a}$ & 4.65 & 4.72 & 4.61 & 4.50 & 4.56 & 4.59 & 4.64 & 4.51 & 4.64 \\
\hline H-9 & 3.92 & 3.76 & 3.78 & 3.86 & 3.74 & 3.84 & 3.90 & 3.88 & 3.65 \\
\hline H-10 & 1.21 & 1.06 & 1.24 & 1.32 & 1.40 & 1.18 & 1.22 & 1.17 & 1.30 \\
\hline
\end{tabular}

\begin{tabular}{|c|c|c|c|c|c|c|c|c|}
\hline & Isomer 1 & Isomer 2 & Isomer 3 & Isomer 4 & Isomer 5 & Isomer 6 & Isomer 7 & Isomer 8 \\
\hline sDP4+ (H data) & $0.06 \%$ & $2.67 \%$ & $\begin{array}{ll}76.63 \% \\
\end{array}$ & $18.21 \%$ & $1.71 \%$ & $0.28 \%$ & $0.28 \%$ & $0.16 \%$ \\
\hline sDP4+ (C data) & $0.00 \%$ & $2.49 \%$ & $42.35 \%$ & $55.16 \%$ & $0.00 \%$ & $0.00 \%$ & $0.00 \%$ & $0.00 \%$ \\
\hline sDP4+ (all data) & $0.00 \%$ & $0.16 \%$ & (1) $76.24 \%$ & $23.60 \%$ & $0.00 \%$ & $0.00 \%$ & $0.00 \%$ & $0.00 \%$ \\
\hline uDP4+ (H data) & $0.00 \%$ & $0.13 \%$ & $41.50 \%$ & $58.36 \%$ & $0.00 \%$ & $0.00 \%$ & $0.00 \%$ & $0.00 \%$ \\
\hline uDP4+ (C data) & $0.01 \%$ & $0.12 \%$ & $41.57 \%$ & $58.30 \%$ & $0.00 \%$ & $0.00 \%$ & $0.00 \%$ & $0.00 \%$ \\
\hline uDP4+ (all data) & $0.00 \%$ & $0.00 \%$ & $33.64 \%$ & $66.36 \%$ & $0.00 \%$ & $0.00 \%$ & $0.00 \%$ & $0.00 \%$ \\
\hline DP4+ (H data) & $0.00 \%$ & $0.01 \%$ & 74.94\% & $25.05 \%$ & $0.00 \%$ & $0.00 \%$ & $\mathbf{0 . 0 0 \%}$ & $0.00 \%$ \\
\hline DP4+ (C data) & $0.00 \%$ & $0.01 \%$ & $35.37 \%$ & $64.62 \%$ & $0.00 \%$ & $0.00 \%$ & $0.00 \%$ & $0.00 \%$ \\
\hline DP4+ (all data) & $0.00 \%$ & $0.00 \%$ & $62.09 \%$ & $37.91 \%$ & $0.00 \%$ & $0.00 \%$ & \begin{tabular}{|l|l|}
$0.00 \%$ \\
\end{tabular} & $0.00 \%$ \\
\hline
\end{tabular}

Figure S9: DP4+ results obtained by correlating the experimental NMR data reported for natural diplopyrone with the NMR data of compounds 1-8 calculated at the PCM/mPW1PW91/6-31+G**//B3LYP/6-31G* level of theory. 
Table S10: Weighted sotropic shielding values and scaled chemical shifts obtained by fitting the B3LYP/6-31G**//MMFF shielding values with the experimental NMR data reported for synthetic diplopyrone.

\begin{tabular}{|c|c|c|c|c|c|c|c|c|c|}
\hline \multirow[b]{2}{*}{ Atom } & \multirow[b]{2}{*}{ Exp } & \multicolumn{4}{|c|}{ Isotropic shielding values } & \multicolumn{4}{|c|}{ Scaled chemical shifts } \\
\hline & & Isomer 1 & Isomer 2 & Isomer 3 & Isomer 4 & Isomer 1 & Isomer 2 & Isomer 3 & Isomer 4 \\
\hline C-2 & 161.7 & 36.0662 & 36.0815 & 36.4882 & 36.6556 & 159.7 & 159.5 & 160.1 & 159.5 \\
\hline C-3 & 123.3 & 69.4392 & 69.4871 & 71.5642 & 72.5174 & 124.1 & 124.0 & 122.8 & 121.4 \\
\hline C-4 & 144.2 & 52.0629 & 52.0565 & 51.9680 & 49.6239 & 142.7 & 142.5 & 143.6 & 145.7 \\
\hline C-4a & 64.9 & 124.4485 & 124.3817 & 126.8737 & 126.7810 & 65.4 & 65.7 & 63.9 & 63.6 \\
\hline C-6 & 74.8 & 115.6188 & 115.8656 & 114.4213 & 114.9183 & 74.8 & 74.7 & 77.1 & 76.2 \\
\hline C-7 & 129.6 & 63.4782 & 63.2418 & 63.9437 & 64.6580 & 130.5 & 130.6 & 130.9 & 129.7 \\
\hline C-8 & 124.7 & 66.5952 & 66.1485 & 68.4602 & 66.9180 & 127.1 & 127.6 & 126.1 & 127.3 \\
\hline C-8a & 70.0 & 121.0838 & 121.0252 & 122.0565 & 121.4391 & 69.0 & 69.2 & 69.0 & 69.3 \\
\hline C-9 & 69.7 & 119.2420 & 119.8170 & 120.6547 & 120.4699 & 71.0 & 70.5 & 70.5 & 70.3 \\
\hline C-10 & 18.7 & 169.5244 & 169.9644 & 170.2449 & 169.3624 & 17.3 & 17.2 & 17.7 & 18.3 \\
\hline & & & & & & - & - & - & - \\
\hline $\mathrm{H}-3$ & 6.07 & 25.6876 & 25.6867 & 26.0002 & 25.9458 & 5.90 & 5.90 & 5.91 & 5.79 \\
\hline $\mathrm{H}-4$ & 6.88 & 24.8429 & 24.8369 & 25.1706 & 24.9461 & 6.73 & 6.74 & 6.73 & 6.79 \\
\hline $\mathrm{H}-4 \mathrm{a}$ & 4.92 & 26.7508 & 26.7235 & 27.3932 & 27.0017 & 4.85 & 4.88 & 4.55 & 4.74 \\
\hline H-6 & 4.08 & 27.5200 & 27.5044 & 27.8511 & 27.5904 & 4.10 & 4.10 & 4.10 & 4.16 \\
\hline H-7 & 6.06 & 25.3830 & 25.4651 & 25.5597 & 25.6106 & 6.20 & 6.12 & 6.35 & 6.12 \\
\hline H-8 & 6.04 & 25.3192 & 25.2997 & 25.5709 & 25.4069 & 6.26 & 6.28 & 6.34 & 6.33 \\
\hline H-8a & 4.97 & 26.6244 & 26.6005 & 26.9771 & 26.6735 & 4.98 & 5.00 & 4.95 & 5.07 \\
\hline H-9 & 3.96 & 27.6322 & 27.5768 & 27.9168 & 27.6889 & 3.99 & 4.03 & 4.03 & 4.06 \\
\hline H-10 & 1.25 & 30.4517 & 30.4624 & 30.7240 & 30.5823 & 1.22 & 1.19 & 1.28 & 1.18 \\
\hline
\end{tabular}

\begin{tabular}{|c|c|c|c|c|c|}
\hline \multicolumn{2}{|c|}{ Isomer No } & 1 & 2 & 3 & 4 \\
\hline \multirow{4}{*}{ DP4 (\%) } & $\mathrm{H}$ & 48.25 & 47.85 & 0.61 & 3.30 \\
\cline { 2 - 6 } & $\mathrm{C}$ & 27.33 & 19.68 & 36.39 & 16.59 \\
\cline { 2 - 6 } & $\mathrm{H}+\mathrm{C}$ & 56.42 & 40.30 & 0.94 & 2.34 \\
\cline { 2 - 6 } & $\mathrm{J}$ & - & - & - & - \\
\cline { 2 - 6 } & all data & 56.42 & 40.30 & 0.94 & 2.34 \\
\hline
\end{tabular}

Figure S10: DP4 results obtained by correlating the experimental NMR data reported for synthetic diplopyrone with the NMR data of compounds 1-8 obtained by fitting the B3LYP/6-31G**//MMFF shielding values with the experimental NMR data reported for synthetic diplopyrone. 
Table S11: Weighted sotropic shielding values and scaled chemical shifts obtained by fitting the B3LYP/6-31G**//MMFF shielding values with the experimental NMR data reported for natural diplopyrone.

\begin{tabular}{|c|c|c|c|c|c|c|c|c|c|}
\hline \multirow[b]{2}{*}{ Atom } & \multirow[b]{2}{*}{ Exp } & \multicolumn{4}{|c|}{ Isotropic shielding values } & \multicolumn{4}{|c|}{ Scaled chemical shifts } \\
\hline & & Isomer 1 & Isomer 2 & Isomer 3 & Isomer 4 & Isomer 1 & Isomer 2 & Isomer 3 & Isomer 4 \\
\hline C-2 & 162.7 & 35.3828 & 35.4144 & 35.4675 & 35.5656 & 160.8 & 160.6 & 160.8 & 160.5 \\
\hline C-3 & 124.8 & 68.9458 & 69.4452 & 69.3138 & 69.5554 & 125.2 & 124.9 & 124.5 & 124.5 \\
\hline C-4 & 140.0 & 54.8152 & 54.8275 & 54.3974 & 55.1192 & 140.2 & 140.2 & 140.5 & 139.8 \\
\hline C-4a & 64.9 & 126.9701 & 127.5862 & 124.9727 & 125.6191 & 63.7 & 63.8 & 64.6 & 65.0 \\
\hline C-6 & 78.9 & 114.5818 & 115.2841 & 112.2031 & 112.6607 & 76.9 & 76.7 & 78.4 & 78.7 \\
\hline $\mathrm{C}-7$ & 132.6 & 61.3064 & 62.1710 & 60.8493 & 61.4499 & 133.3 & 132.5 & 133.6 & 133.1 \\
\hline C-8 & 123.0 & 68.6214 & 67.4868 & 68.9626 & 67.9090 & 125.6 & 126.9 & 124.8 & 126.2 \\
\hline C-8a & 69.7 & 122.0805 & 122.2429 & 121.2034 & 121.8459 & 68.9 & 69.4 & 68.7 & 69.0 \\
\hline C-9 & 69.0 & 120.5891 & 121.2278 & 120.1236 & 121.0956 & 70.5 & 70.4 & 69.8 & 69.8 \\
\hline C-10 & 17.8 & 169.8473 & 171.1068 & 168.6271 & 170.8247 & 18.3 & 18.0 & 17.7 & 17.0 \\
\hline $\mathrm{H}-3$ & 6.23 & 25.6905 & 25.7557 & 25.6630 & 25.7621 & 6.00 & 5.98 & 6.03 & 6.05 \\
\hline H-4 & 6.88 & 25.0195 & 25.0742 & 24.9885 & 25.0568 & 6.68 & 6.67 & 6.70 & 6.75 \\
\hline $\mathrm{H}-4 \mathrm{a}$ & 4.09 & 27.3224 & 27.2799 & 27.5473 & 27.7385 & 4.35 & 4.43 & 4.14 & 4.09 \\
\hline H-6 & 4.16 & 27.5798 & 27.5521 & 27.7696 & 27.7426 & 4.09 & 4.16 & 3.92 & 4.08 \\
\hline H-7 & 6.18 & 25.3232 & 25.4780 & 25.2153 & 25.5342 & 6.38 & 6.26 & 6.48 & 6.28 \\
\hline H-8 & 6.14 & 25.4003 & 25.3840 & 25.3798 & 25.4577 & 6.30 & 6.35 & 6.31 & 6.35 \\
\hline H-8a & 4.65 & 27.0710 & 27.0820 & 27.0181 & 27.1940 & 4.61 & 4.63 & 4.67 & 4.63 \\
\hline H-9 & 3.92 & 27.7283 & 27.7310 & 27.7146 & 27.7468 & 3.94 & 3.97 & 3.98 & 4.08 \\
\hline H-10 & 1.21 & 30.5425 & 30.6492 & 30.4691 & 30.6852 & 1.10 & 1.02 & 1.22 & 1.16 \\
\hline
\end{tabular}

\begin{tabular}{|c|c|c|c|c|c|}
\hline \multicolumn{2}{|c|}{ Isomer No } & 1 & 2 & 3 & 4 \\
\hline \multirow{4}{*}{ DP4 (\%) } & $\mathrm{H}$ & 7.25 & 2.67 & 8.39 & 81.70 \\
\cline { 2 - 6 } & $\mathrm{C}$ & 9.63 & 6.42 & 54.55 & 29.40 \\
\cline { 2 - 6 } & $\mathrm{H}+\mathrm{C}$ & 2.37 & 0.58 & 15.54 & 81.51 \\
\cline { 2 - 6 } & $\mathrm{J}$ & - & - & - & - \\
\cline { 2 - 6 } & all data & 2.37 & 0.58 & 15.54 & 81.51 \\
\hline
\end{tabular}

Figure S11: DP4 results obtained by correlating the experimental NMR data reported for natural diplopyrone with the NMR data of compounds 1-8 obtained by fitting the B3LYP/6-31G**//MMFF shielding values with the experimental NMR data reported for natural diplopyrone. 
Table S12: Weighted sotropic shielding values and scaled chemical shifts obtained by fitting the PCM/mPW1PW91/6$31+\mathrm{G}^{* * / / B 3 L Y P} / 6-31 \mathrm{G}^{*}$ shielding values with the experimental NMR data reported for synthetic diplopyrone.

\begin{tabular}{|c|c|c|c|c|c|c|c|c|c|}
\hline \multirow[b]{2}{*}{ Atom } & \multirow[b]{2}{*}{$\operatorname{Exp}$} & \multicolumn{4}{|c|}{ Isotropic shielding values } & \multicolumn{4}{|c|}{ Scaled chemical shifts } \\
\hline & & Isomer 1 & Isomer 2 & Isomer 3 & Isomer 4 & Isomer 1 & Isomer 2 & Isomer 3 & Isomer 4 \\
\hline C-2 & 161.7 & 37.1909 & 37.0262 & 36.4440 & 37.7804 & 160.0 & 159.8 & 160.6 & 159.7 \\
\hline C-3 & 123.3 & 74.2766 & 73.7692 & 72.2943 & 75.3343 & 121.8 & 122.1 & 123.6 & 121.2 \\
\hline C-4 & 144.2 & 50.6542 & 51.1470 & 54.8617 & 50.0851 & 146.1 & 145.3 & 141.6 & 147.0 \\
\hline $\mathrm{C}-4 \mathrm{a}$ & 64.9 & 128.9035 & 128.8966 & 129.1817 & 129.4142 & 65.5 & 65.5 & 65.0 & 65.8 \\
\hline C-6 & 74.8 & 119.3696 & 118.5477 & 115.9195 & 117.2215 & 75.3 & 76.1 & 78.7 & 78.3 \\
\hline C-7 & 129.6 & 66.6905 & 65.2537 & 62.3298 & 67.5885 & 129.6 & 130.9 & 133.9 & 129.1 \\
\hline C-8 & 124.7 & 71.5665 & 71.6468 & 73.7449 & 72.3711 & 124.6 & 124.3 & 122.1 & 124.2 \\
\hline $\mathrm{C}-8 \mathrm{a}$ & 70.0 & 124.4170 & 124.2273 & 124.6121 & 124.3749 & 70.1 & 70.3 & 69.7 & 70.9 \\
\hline C-9 & 69.7 & 122.9540 & 124.1278 & 123.8286 & 126.0066 & 71.6 & 70.4 & 70.5 & 69.3 \\
\hline $\mathrm{C}-10$ & 18.7 & 176.2011 & 176.2216 & 176.8221 & 177.8958 & 16.8 & 16.9 & 15.9 & 16.1 \\
\hline $\mathrm{H}-3$ & 6.07 & 25.3633 & 25.2412 & 25.1326 & 25.4845 & 5.91 & 5.98 & 6.16 & 6.00 \\
\hline $\mathrm{H}-4$ & 6.88 & 24.2436 & 24.1535 & 24.1970 & 24.3917 & 6.94 & 6.98 & 7.00 & 6.99 \\
\hline $\mathrm{H}-4 \mathrm{a}$ & 4.92 & 26.4513 & 26.4841 & 27.3934 & 27.0119 & 4.92 & 4.83 & 4.11 & 4.61 \\
\hline H-6 & 4.08 & 27.3298 & 27.2923 & 27.1571 & 27.6114 & 4.11 & 4.08 & 4.32 & 4.07 \\
\hline H-7 & 6.06 & 25.0096 & 25.0014 & 24.8810 & 25.2356 & 6.24 & 6.20 & 6.38 & 6.22 \\
\hline H-8 & 6.04 & 25.1349 & 25.0231 & 25.0196 & 25.2958 & 6.12 & 6.18 & 6.26 & 6.17 \\
\hline $\mathrm{H}-8 \mathrm{a}$ & 4.97 & 26.6542 & 26.6099 & 26.8512 & 26.6765 & 4.73 & 4.71 & 4.60 & 4.92 \\
\hline H-9 & 3.96 & 27.5189 & 27.4879 & 27.5863 & 27.8419 & 3.94 & 3.90 & 3.94 & 3.86 \\
\hline $\mathrm{H}-10$ & 1.25 & 30.3885 & 30.2257 & 30.3245 & 30.5469 & 1.31 & 1.37 & 1.46 & 1.40 \\
\hline
\end{tabular}

\begin{tabular}{|c|c|c|c|c|}
\hline & Isomer 1 & Isomer 2 & Isomer 3 & Isomer 4 \\
\hline sDP4+ (H data) & $77.75 \%$ & $15.80 \%$ & $0.00 \%$ & $6.45 \%$ \\
\hline sDP4+ (C data) & $44.27 \%$ & $55.21 \%$ & $0.04 \%$ & $0.48 \%$ \\
\hline sDP4+ (all data) & 79.72\% & $20.21 \%$ & $0.00 \%$ & $0.07 \%$ \\
\hline uDP4+ (H data) & $75.62 \%$ & $23.37 \%$ & $0.16 \%$ & $0.84 \%$ \\
\hline uDP4+ (C data) & $47.79 \%$ & $50.54 \%$ & $0.35 \%$ & $1.33 \%$ \\
\hline uDP4+ (all data) & $75.35 \%$ & $24.63 \%$ & $0.00 \%$ & $0.02 \%$ \\
\hline DP4+ (H data) & 94.01\% & $5.91 \%$ & $0.00 \%$ & $0.09 \%$ \\
\hline DP4+ (C data) & $43.12 \%$ & $56.87 \%$ & $0.00 \%$ & $0.01 \%$ \\
\hline DP4+ (all data) & क्या $92.35 \%$ & 7.65\% & $0.00 \%$ & $0.00 \%$ \\
\hline
\end{tabular}

Figure S12: DP4 results obtained by correlating the experimental NMR data reported for synthetic diplopyrone with the NMR data of compounds 1-8 obtained by fitting the B3LYP/6-31G**//MMFF shielding values with the experimental NMR data reported for synthetic diplopyrone. 
Table S13: Weighted sotropic shielding values and scaled chemical shifts obtained by fitting the PCM/mPW1PW91/6$31+\mathrm{G}^{* * / / B} 3 \mathrm{LYP} / 6-31 \mathrm{G}^{*}$ shielding values with the experimental NMR data reported for natural diplopyrone.

\begin{tabular}{|c|c|c|c|c|c|c|c|c|c|}
\hline \multirow[b]{2}{*}{ Atom } & \multirow[b]{2}{*}{ Exp } & \multicolumn{4}{|c|}{ Isotropic shielding values } & \multicolumn{4}{|c|}{ Scaled chemical shifts } \\
\hline & & Isomer 1 & Isomer 2 & Isomer 3 & Isomer 4 & Isomer 1 & Isomer 2 & Isomer 3 & Isomer 4 \\
\hline C-2 & 162.7 & 36.9748 & 36.7901 & 36.4239 & 36.7050 & 160.8 & 160.6 & 160.6 & 160.5 \\
\hline C-3 & 124.8 & 73.9469 & 73.4762 & 72.2545 & 72.7163 & 122.9 & 123.1 & 123.8 & 123.5 \\
\hline C-4 & 140.0 & 52.5501 & 53.0776 & 54.8314 & 54.2032 & 144.8 & 144.0 & 141.7 & 142.6 \\
\hline $\mathrm{C}-4 \mathrm{a}$ & 64.9 & 130.9893 & 130.8683 & 129.1107 & 129.2233 & 64.4 & 64.3 & 65.2 & 65.5 \\
\hline C-6 & 78.9 & 118.7549 & 117.9737 & 115.8557 & 116.2628 & 76.9 & 77.5 & 78.9 & 78.8 \\
\hline C-7 & 132.6 & 64.3900 & 64.2979 & 62.2946 & 63.6253 & 132.7 & 132.5 & 134.0 & 132.9 \\
\hline C-8 & 123.0 & 74.2647 & 73.2114 & 73.7051 & 73.1605 & 122.5 & 123.4 & 122.3 & 123.1 \\
\hline $\mathrm{C}-8 \mathrm{a}$ & 69.7 & 125.5580 & 125.4015 & 124.5434 & 124.5880 & 69.9 & 69.9 & 69.9 & 70.3 \\
\hline C-9 & 69.0 & 124.9883 & 124.8905 & 123.7599 & 124.9725 & 70.5 & 70.4 & 70.7 & 69.9 \\
\hline $\mathrm{C}-10$ & 17.8 & 176.1684 & 176.4496 & 176.7233 & 177.1138 & 18.1 & 17.7 & 16.2 & 16.3 \\
\hline $\mathrm{H}-3$ & 6.23 & 25.4120 & 25.3101 & 25.1188 & 25.2110 & 5.97 & 6.01 & 6.09 & 6.06 \\
\hline $\mathrm{H}-4$ & 6.88 & 24.3744 & 24.2937 & 24.1837 & 24.2573 & 6.94 & 6.98 & 6.96 & 6.94 \\
\hline $\mathrm{H}-4 \mathrm{a}$ & 4.09 & 27.0603 & 26.8101 & 27.3783 & 27.3417 & 4.42 & 4.57 & 4.01 & 4.09 \\
\hline H-6 & 4.16 & 27.5142 & 27.4696 & 27.1422 & 27.2591 & 4.00 & 3.94 & 4.23 & 4.17 \\
\hline H-7 & 6.18 & 24.9068 & 25.0741 & 24.8672 & 24.9595 & 6.44 & 6.23 & 6.33 & 6.29 \\
\hline H-8 & 6.14 & 25.2475 & 25.1092 & 25.0059 & 25.0323 & 6.12 & 6.20 & 6.20 & 6.23 \\
\hline $\mathrm{H}-8 \mathrm{a}$ & 4.65 & 26.9114 & 26.8572 & 26.8364 & 26.8356 & 4.56 & 4.53 & 4.51 & 4.56 \\
\hline H-9 & 3.92 & 27.7459 & 27.6154 & 27.5713 & 27.6202 & 3.78 & 3.81 & 3.83 & 3.83 \\
\hline $\mathrm{H}-10$ & 1.21 & 30.4584 & 30.3504 & 30.3077 & 30.3813 & 1.24 & 1.19 & 1.31 & 1.28 \\
\hline
\end{tabular}

\begin{tabular}{|c|c|c|c|c|}
\hline & Isomer 1 & Isomer 2 & Isomer 3 & Isomer 4 \\
\hline sDP4+ (H data) & $0.17 \%$ & $0.21 \%$ & $13.70 \%$ & $85.91 \%$ \\
\hline sDP4+ (C data) & $2.37 \%$ & $9.83 \%$ & $33.15 \%$ & $54.65 \%$ \\
\hline sDP4+ (all data) & $0.01 \%$ & $0.04 \%$ & $8.82 \%$ & 91.13\% \\
\hline uDP4+ (H data) & $0.44 \%$ & $6.11 \%$ & $8.00 \%$ & $85.45 \%$ \\
\hline uDP4+ (C data) & $0.56 \%$ & $1.40 \%$ & $52.07 \%$ & $45.97 \%$ \\
\hline uDP4+ (all data) & $0.01 \%$ & $0.20 \%$ & $9.57 \%$ & 90.23\% \\
\hline DP4+ (H data) & $0.00 \%$ & $0.02 \%$ & $1.47 \%$ & 98.51\% \\
\hline DP4+ (C data) & $0.03 \%$ & $0.32 \%$ & $40.58 \%$ & $59.06 \%$ \\
\hline DP4+ (all data) & $0.00 \%$ & $\mathbf{0 . 0 0 \%}$ & $1.02 \%$ & 98.98\% \\
\hline
\end{tabular}

Figure S13: DP4+ results obtained by correlating the experimental NMR data reported for natural diplopyrone with the NMR data of compounds 1-8 obtained by fitting the B3LYP/6-31G**//MMFF shielding values with the experimental NMR data reported for natural diplopyrone. 

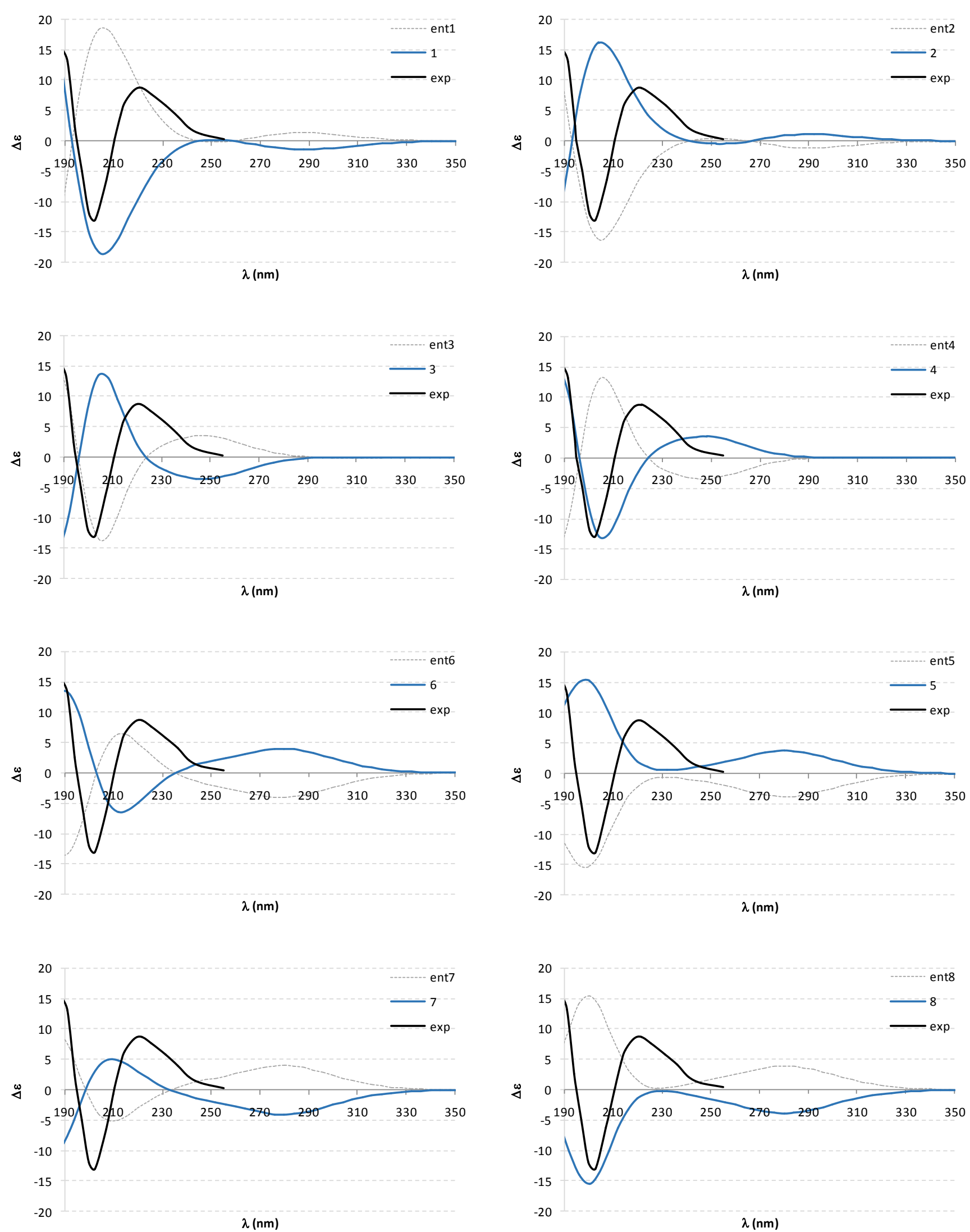

Figure S14: ECD spectra computed for 1-8 (and their corresponding enantiomers) at the B3LYP/6-31G* level of theory. The experimental ECD shown has been graphically adapted from the original reference and is provided for qualitatively comparison only. 

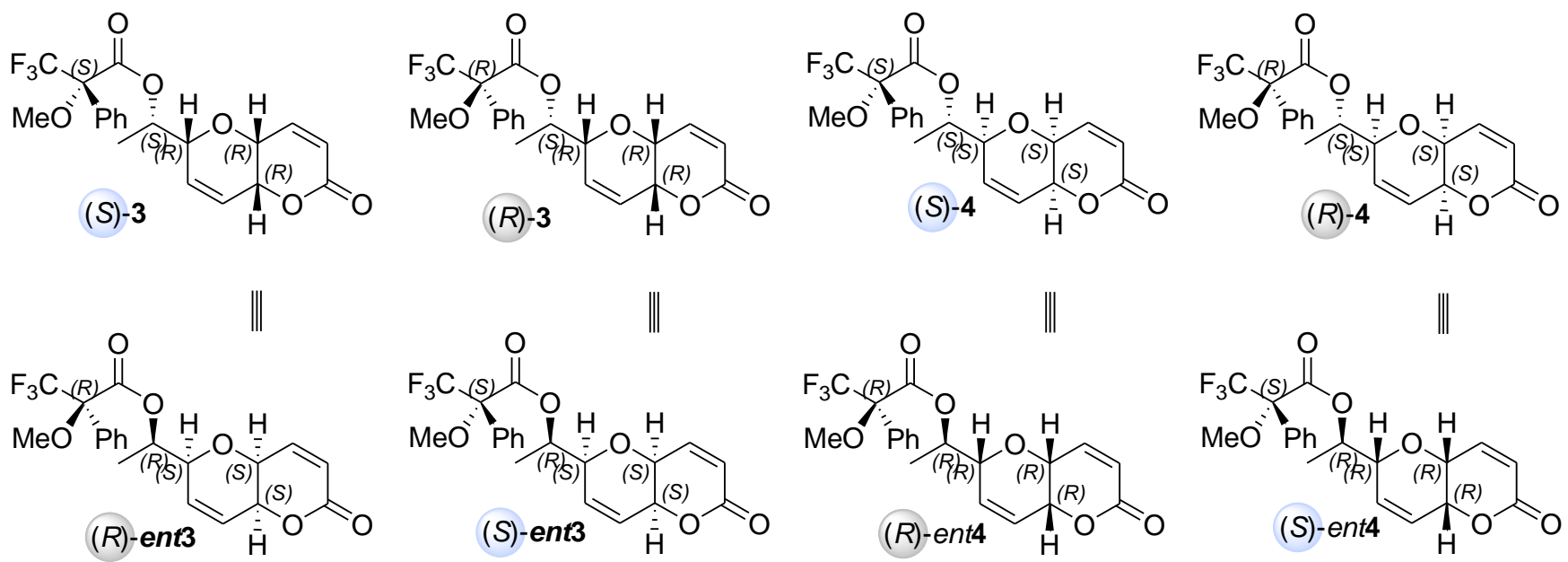

Figure S15: Equivalences between MTPA derivatives of $\mathbf{3}$ and 4. Note that each enantiomeric pair (e.g. $(S)$-3 and $(R)$-ent $\mathbf{3})$ display the same computed NMR shifts.

Table S14: Bolzmtann-averaged isotropic shielding values computed for selected protons of compounds $(R)$-MTPA-3, $(S)$ MTPA-3, $(R)$-MTPA-4 and $(S)$-MPTA-4 at the PCM/mPW1PW91/6-31+G**//B3LYP/6-31G* level of theory.

\begin{tabular}{ccccc}
\hline Atom & $\begin{array}{c}(R) \text {-MPTA-4 or } \\
(S) \text {-MPTA-ent } 4\end{array}$ & $\begin{array}{c}(R) \text {-MPTA-3 or } \\
(S) \text {-MPTA-ent3 }\end{array}$ & $\begin{array}{c}(S) \text {-MPTA-4 or } \\
(R) \text {-MPTA-ent } 4\end{array}$ & $\begin{array}{c}(S) \text {-MPTA-3 or } \\
(R) \text {-MPTA-ent } 3\end{array}$ \\
\hline H-3 & 25.2070 & 25.2499 & 25.1839 & 25.1685 \\
H-4 & 24.4092 & 24.4660 & 24.2598 & 24.2546 \\
H-4a & 27.6159 & 27.6472 & 27.4108 & 27.4444 \\
H-6 & 27.3532 & 27.2206 & 27.0376 & 26.9387 \\
H-7 & 25.1407 & 25.2654 & 24.9009 & 25.0007 \\
H-8 & 25.1087 & 25.1496 & 25.0293 & 25.0033 \\
H-8a & 27.0356 & 27.0027 & 26.9075 & 26.9067 \\
H-9 & 26.1023 & 26.4677 & 26.2195 & 26.2885 \\
H-10 & 30.2705 & 30.2126 & 30.4437 & 30.3521 \\
\hline
\end{tabular}


Table S15: Experimental chemical shifts reported for $(R)$-MTPA-ND and $(S)$-MTPA-ND and scaled chemical shifts computed for the corresponding 3-derivatives at the PCM/mPW1PW91/6-31+G**//B3LYP/6-31G* level of theory.

\begin{tabular}{|c|c|c|c|c|c|c|}
\hline Atom & $\begin{array}{c}\mathbf{E x p} \\
(R)-\text { MPTA-ND }\end{array}$ & $(R)$-MPTA-3 & $(R)$-MPTA-ent3 & $\begin{array}{c}\mathbf{E x p} \\
(S) \text {-MPTA-ND }\end{array}$ & $(S)$-MPTA-ent3 & $(S)$-MPTA-3 \\
\hline $\mathrm{H}-3$ & 6.24 & 6.37 & 6.25 & 6.19 & 6.12 & 6.01 \\
\hline $\mathrm{H}-4$ & 6.85 & 7.17 & 7.14 & 6.78 & 6.85 & 6.83 \\
\hline $\mathrm{H}-4 \mathrm{a}$ & 4.07 & 3.94 & 4.04 & 4.00 & 3.88 & 3.98 \\
\hline H-6 & 4.33 & 4.37 & 4.53 & 4.23 & 4.28 & 4.43 \\
\hline $\mathrm{H}-7$ & 6.10 & 6.36 & 6.42 & 5.97 & 6.11 & 6.16 \\
\hline $\mathrm{H}-8$ & 6.96 & 6.48 & 6.42 & 6.10 & 6.22 & 6.16 \\
\hline $\mathrm{H}-8 \mathrm{a}$ & 4.65 & 4.59 & 4.57 & 4.60 & 4.49 & 4.46 \\
\hline H-9 & 5.27 & 5.14 & 5.17 & 5.18 & 4.99 & 5.01 \\
\hline $\mathrm{H}-10$ & 1.29 & 1.34 & 1.22 & 1.38 & 1.49 & 1.38 \\
\hline
\end{tabular}

\begin{tabular}{|c|c|c|}
\cline { 2 - 3 } & R-3 & R-ent3 \\
\hline SDP4+ (H data) & $57.10 \%$ & $42.90 \%$ \\
\hline SDP4+ (C data) & - & - \\
\hline SDP4+ (all data) & $57.10 \%$ & $42.90 \%$ \\
\hline uDP4+ (H data) & $13.50 \%$ & - \\
\hline uDP4+ (C data) & - & - \\
\hline uDP4+ (all data) & $13.50 \%$ & $-50 \%$ \\
\hline DP4+ (H data) & $17.20 \%$ & $86.50 \%$ \\
\hline DP4+ (C data) & - & - \\
\hline DP4+ (all data) & $\mathbf{1 7 . 2 0 \%}$ & $-80 \%$ \\
\hline
\end{tabular}

\begin{tabular}{|c|c|c|}
\hline & S-ent3 & S-3 \\
\hline sDP4+ (H data) & $60.82 \%$ & $39.18 \%$ \\
\hline SDP4+ (C data) & - & - \\
\hline sDP4+ (all data) & $60.82 \%$ & $39.18 \%$ \\
\hline uDP4+ (H data) & (99.97\% & $0.03 \%$ \\
\hline uDP4+ (C data) & - & - \\
\hline uDP4+ (all data) & 的 $99.97 \%$ & $0.03 \%$ \\
\hline DP4+ (H data) & 99.98\% & $0.02 \%$ \\
\hline DP4+ (C data) & - & - \\
\hline DP4+ (all data) & and $99.98 \%$ & $0.02 \%$ \\
\hline
\end{tabular}

Figure S16: DP4+ results obtained by correlating the experimental NMR data reported for $(R)$-MTPA-ND and $(S)$-MTPAND with the NMR data of the corresponding 3 derivatives computed at the PCM/mPW1PW91/6-31+G**//B3LYP/6-31G* level of theory.

Table S16: Experimental chemical shifts reported for $(R)$-MTPA-ND and $(S)$-MTPA-ND and scaled chemical shifts computed for the corresponding 4-derivatives at the PCM/mPW1PW91/6-31+G**//B3LYP/6-31G* level of theory.

\begin{tabular}{|c|c|c|c|c|c|c|}
\hline Atom & $\begin{array}{c}\mathbf{E x p} \\
(R) \text {-MPTA-ND }\end{array}$ & (R)-MPTA-4 & (R)-MPTA-ent4 & $\begin{array}{c}\text { Exp } \\
(S) \text {-MPTA-ND }\end{array}$ & $(S)$-MPTA-ent4 & (S)-MPTA-4 \\
\hline $\mathrm{H}-3$ & 6.24 & 6.33 & 6.23 & 6.19 & 6.08 & 5.98 \\
\hline $\mathrm{H}-4$ & 6.85 & 7.12 & 7.11 & 6.78 & 6.80 & 6.79 \\
\hline $\mathrm{H}-4 \mathrm{a}$ & 4.07 & 3.96 & 4.10 & 4.00 & 3.90 & 4.03 \\
\hline H-6 & 4.33 & 4.21 & 4.45 & 4.23 & 4.14 & 4.36 \\
\hline H-7 & 6.10 & 6.40 & 6.50 & 5.97 & 6.14 & 6.23 \\
\hline $\mathrm{H}-8$ & 6.96 & 6.43 & 6.37 & 6.10 & 6.17 & 6.12 \\
\hline $\mathrm{H}-8 \mathrm{a}$ & 4.65 & 4.53 & 4.58 & 4.60 & 4.43 & 4.47 \\
\hline $\mathrm{H}-9$ & 5.27 & 5.45 & 5.24 & 5.18 & 5.27 & 5.07 \\
\hline $\mathrm{H}-10$ & 1.29 & 1.34 & 1.20 & 1.38 & 1.50 & 1.37 \\
\hline
\end{tabular}

\begin{tabular}{|c|c|c|}
\hline & R-4 & R-ent4 \\
\hline sDP4+ (H data) & $11.62 \%$ & (口) $88.38 \%$ \\
\hline sDP4+ (C data) & - & - \\
\hline sDP4+ (all data) & $11.62 \%$ & (口) $88.38 \%$ \\
\hline uDP4+ (H data) & $8086.95 \%$ & $13.05 \%$ \\
\hline uDP4+ (C data) & - & - \\
\hline uDP4+ (all data) & (1) $86.95 \%$ & $13.05 \%$ \\
\hline DP4+ (H data) & $46.70 \%$ & $53.30 \%$ \\
\hline DP4+ (C data) & - & - \\
\hline DP4+ (all data) & $46.70 \%$ & $53.30 \%$ \\
\hline
\end{tabular}

\begin{tabular}{|c|c|c|}
\hline & S-ent4 & S-4 \\
\hline sDP4+ (H data) & $41.42 \%$ & $58.58 \%$ \\
\hline sDP4+ (C data) & - & - \\
\hline sDP4+ (all data) & $41.42 \%$ & $58.58 \%$ \\
\hline uDP4+ (H data) & $99.98 \%$ & $0.02 \%$ \\
\hline uDP4+ (C data) & - & - \\
\hline uDP4+ (all data) & $99.98 \%$ & $0.02 \%$ \\
\hline DP4+ (H data) & $99.97 \%$ & $0.03 \%$ \\
\hline DP4+ (C data) & - & - \\
\hline DP4+ (all data) & 99.97\% & $0.03 \%$ \\
\hline
\end{tabular}

Figure S17: DP4+ results obtained by correlating the experimental NMR data reported for $(R)$-MTPA-ND and $(S)$-MTPAND with the NMR data of the corresponding 4 derivatives computed at the PCM/mPW1PW91/6-31+G**//B3LYP/6-31G* level of theory. 
Table S17: Experimental chemical shifts reported for $(R)$-MTPA-ND and scaled chemical shifts computed for the corresponding 3 and $\mathbf{4}$ derivatives at the PCM/mPW1PW91/6-31+G**//B3LYP/6-31G* level of theory.

\begin{tabular}{cccccc}
\hline Atom & Exp & $(R)$-MPTA-3 & $(R)$-MPTA-ent3 & $(R)$-MPTA-4 & $(R)$-MPTA-ent4 \\
\hline H-3 & 6.24 & 6.37 & 6.25 & 6.33 & 6.23 \\
H-4 & 6.85 & 7.17 & 7.14 & 7.12 & 7.11 \\
H-4a & 4.07 & 3.94 & 4.04 & 3.96 & 4.10 \\
H-6 & 4.33 & 4.37 & 4.53 & 4.21 & 4.45 \\
H-7 & 6.10 & 6.36 & 6.42 & 6.40 & 6.50 \\
H-8 & 6.96 & 6.48 & 6.42 & 6.43 & 6.37 \\
H-8a & 4.65 & 4.59 & 4.57 & 4.53 & 4.58 \\
H-9 & 5.27 & 5.14 & 5.17 & 5.45 & 5.24 \\
H-10 & 1.29 & 1.34 & 1.22 & 1.34 & 1.20 \\
\hline
\end{tabular}

\begin{tabular}{|c|c|c|c|c|}
\hline & R-3 & R-ent3 & R-4 & R-ent4 \\
\hline sDP4+ (H data) & $26.07 \%$ & $19.59 \%$ & $6.31 \%$ & $48.02 \%$ \\
\hline SDP4+ (C data) & - & - & - & - \\
\hline sDP4+ (all data) & $26.07 \%$ & $19.59 \%$ & $6.31 \%$ & $48.02 \%$ \\
\hline uDP4+ (H data) & $7.64 \%$ & $48.93 \%$ & $37.76 \%$ & $5.67 \%$ \\
\hline uDP4+ (C data) & - & - & - & - \\
\hline uDP4+ (all data) & $7.64 \%$ & $\begin{array}{lll}48.93 \% \\
\end{array}$ & $37.76 \%$ & $5.67 \%$ \\
\hline DP4+ (H data) & $11.94 \%$ & $\begin{array}{l}57.46 \% \\
\ln \end{array}$ & $14.29 \%$ & $16.31 \%$ \\
\hline DP4+ (C data) & - & - & - & - \\
\hline DP4+ (all data) & $11.94 \%$ & $5057.46 \%$ & $14.29 \%$ & $16.31 \%$ \\
\hline
\end{tabular}

Figure S18: DP4+ results obtained by correlating the experimental NMR data reported for $(R)$-MTPA-ND with the NMR data of the corresponding 3 and $\mathbf{4}$ derivatives computed at the PCM/mPW1PW91/6-31+G**//B3LYP/6-31G* level of theory.

Table S18: Experimental chemical shifts reported for $(S)$-MTPA-ND and scaled chemical shifts computed for the corresponding 3 and $\mathbf{4}$ derivatives at the PCM/mPW1PW91/6-31+G**//B3LYP/6-31G* level of theory.

\begin{tabular}{cccccc}
\hline Atom & $\begin{array}{c}\text { Exp } \\
(S) \text {-MPTA-ND }\end{array}$ & $(S)$-MPTA-ent3 & $(S)$-MPTA-3 & $(S)$-MPTA-ent4 & $(S)$-MPTA-4 \\
\hline H-3 & 6.19 & 6.12 & 6.01 & 6.08 & 5.98 \\
H-4 & 6.78 & 6.85 & 6.83 & 6.80 & 6.79 \\
H-4a & 4.00 & 3.88 & 3.98 & 3.90 & 4.03 \\
H-6 & 4.23 & 4.28 & 4.43 & 4.14 & 4.36 \\
H-7 & 5.97 & 6.11 & 6.16 & 6.14 & 6.23 \\
H-8 & 6.10 & 6.22 & 6.16 & 6.17 & 6.12 \\
H-8a & 4.60 & 4.49 & 4.46 & 4.43 & 4.47 \\
H-9 & 5.18 & 4.99 & 5.01 & 5.27 & 5.07 \\
H-10 & 1.38 & 1.49 & 1.38 & 1.50 & 1.37 \\
\hline
\end{tabular}

\begin{tabular}{|c|c|c|c|c|}
\hline & S-ent3 & S-3 & S-ent4 & S-4 \\
\hline sDP4+ (H data) & $20.42 \%$ & $13.15 \%$ & $27.51 \%$ & $38.92 \%$ \\
\hline SDP4+ (C data) & - & - & - & - \\
\hline sDP4+ (all data) & $20.42 \%$ & $13.15 \%$ & $27.51 \%$ & $38.92 \%$ \\
\hline uDP4+ (H data) & 79.68\% & $0.02 \%$ & $20.29 \%$ & $0.00 \%$ \\
\hline uDP4+ (C data) & - & - & - & - \\
\hline uDP4+ (all data) & 79.68\% & $0.02 \%$ & $20.29 \%$ & $0.00 \%$ \\
\hline DP4+ (H data) & 74.44\% & $0.01 \%$ & $25.54 \%$ & $0.01 \%$ \\
\hline DP4+ (C data) & - & - & - & - \\
\hline DP4+ (all data) & 74.44\% & $0.01 \%$ & $25.54 \%$ & $0.01 \%$ \\
\hline
\end{tabular}

Figure S19: DP4+ results obtained by correlating the experimental NMR data reported for $(S)$-MTPA-ND with the NMR data of the corresponding 3 and $\mathbf{4}$ derivatives computed at the PCM/mPW1PW91/6-31+G**//B3LYP/6-31G* level of theory. 
Cartesian Coordinates of the most stable conformations found for compounds $1-8$ at the B3LYP/6-31G**//MMFF level of theory.

\section{$01 \mathrm{c} 01$}

MMFF Geometry

O $-0.8532380 .855608-0.894676$

C $1.016907-0.751324-0.891157$

C $-0.897230-1.0275770 .659878$

C $0.211717-1.5366630 .103525$

C -1.4373450 .3438660 .327078$

C $0.5692910 .721985-0.990985$

C $1.2558991 .594105 \quad 0.010638$

C $2.367110 \quad 1.187504 \quad 0.634324$

C $3.025623-0.0917350 .316218$

O $2.415646-0.902393-0.580308$

H $0.849017 \quad 1.103484-1.980847$

H $0.861791-1.225534-1.868198$

C $-2.9736310 .391320 \quad 0.110577$

C $-3.790325-0.0249461 .324548$

O $4.109888-0.3649850 .814632$

H -1.1892201 .0292061 .146893$

O $-3.351484-0.433175-0.998921$

H -1.432885 -1.620649 1.396081

H $0.551250-2.5357910 .361678$

H 0.8364562 .5733490 .217481

H 2.8776641 .8101291 .359963

H $-3.2518551 .415932-0.165366$

H -3.5088690 .5510032 .211014$

H $-3.681255-1.0923501 .540901$

H -4.8567370 .1372131 .131613$

$\mathrm{H}-2.709956-0.225634-1.704895$

SCF Energy $\left(B 3 L Y P / 6-31 G^{* *}\right)=-689.145177605$

\section{1_c02}

MMFF Geometry

O - $0.386652-0.670589-0.841609$

$\begin{array}{llll}C & 0.899951 & 0.640558 & 0.811803\end{array}$

C -1.226653 $1.513804-0.115012$

C -0.1139381 .7260840 .603755$

C -1.532184 $0.204687-0.807611$

C $0.332549-0.7315240 .391878$

C $1.448907-1.6993240 .178666$

C $2.703135-1.289668-0.042152$

C $3.0476350 .136231-0.174715$

$\begin{array}{lllll}\text { O } & 2.068982 & 1.037665 & 0.068214\end{array}$

H $-0.314715-1.1238501 .182465$

H $1.1829040 .615593 \quad 1.871996$

C $-2.784129-0.543195-0.266581$

C $-4.0799480 .216449-0.530357$

O $4.1763350 .467331-0.515637$

H -1.725293 $0.438443-1.862099$

O $-2.685365-0.7892151 .142424$

H $-1.9407332 .322558-0.241129$

H 0.0810712 .6965101 .048907

H $1.212065-2.7578420 .195228$

H $3.513264-1.988577-0.212296$

H - $2.833552-1.525826-0.750795$

H $-4.1993040 .444434-1.593730$

H $-4.120447 \quad 1.1497450 .040840$

H $-4.940799-0.379486-0.207491$

H -3.472803 -1.297841 1.402909

SCF Energy $\left(B 3 L Y P / 6-31 G^{* \star}\right)=-689.142575479$

01_c03

MMFF Geometry

O $-0.387692-0.717798-0.827912$

C 0.8819520 .6576350 .780788

C -1.247934 $1.481580-0.195418$

C -0.1411921 .7270280 .522622$

C -1.546316 $0.137953-0.823509$

C $0.333398-0.7350470 .404809$

C $1.460267-1.6954740 .221062$

C $2.711235-1.279644-0.006228$

C $3.0427240 .145167-0.175416$

$\begin{array}{llll}\text { O } & 2.052739 & 1.042791 & 0.034510\end{array}$

H $-0.310007-1.1105741 .206742$
H 1.1547450 .6741121 .843721

C $-2.777512-0.590083-0.211331$

C $-4.096748 \quad 0.096949-0.548299$

O $4.1703550 .479483-0.516090$

H $-1.7596440 .313153-1.885105$

O $-2.684933-0.7055981 .215916$

H $-1.9560112 .285717-0.377564$

H 0.0560632 .7202740 .914718

H $1.233964-2.7558510 .264897$

H $3.528550-1.975327-0.154511$

H $-2.801683-1.615742-0.596930$

H $-4.2344250 .184486-1.630150$

H $-4.1557421 .095553-0.102945$

H - $-4.935813-0.474975-0.137014$

$\mathrm{H}-2.743000 \quad 0.1917621 .588269$

SCF Energy $\left(B 3 L Y P / 6-31 G^{* *}\right)=-689.145131267$

01_c04

MM̄MF Geometry

$\begin{array}{llll}\text { O } & 1.007171 & 0.615856 & 0.710576\end{array}$

C $-0.976449-0.8513710 .780028$

C $0.733218-1.134242-0.990790$

C $-0.354711-1.603104-0.363272$

C $1.4296550 .140835-0.588281$

C $-0.404840 \quad 0.5734810 .941764$

C $-1.115068 \quad 1.5682010 .080554$

C $-2.3159371 .295836-0.442094$

C $-3.0429500 .050851-0.136913$

O $-2.408757-0.872561 \quad 0.624193$

H $-0.5577830 .893816 \quad 1.979831$

H $-0.752819-1.4193451 .691536$

C $2.972414-0.000444-0.543770$

C $3.473033-0.9889650 .505673$

O $-4.194793-0.099369-0.523351$

H $1.1878840 .923670-1.318146$

O $3.549317 \quad 1.279049-0.245882$

H $1.138506-1.680840-1.839081$

H $-0.816315-2.536131-0.673921$

H $-0.6367462 .523810-0.109125$

H $-2.8444582 .007827-1.065476$

H $3.346989-0.300735-1.528874$

H $3.107704-2.0021670 .315630$

H $3.170208-0.6887291 .514563$

H $4.568445-1.0136830 .506703$

H $3.074686 \quad 1.6022280 .544678$

SCF Energy $\left(B 3 L Y P / 6-31 G^{* *}\right)=-689.145402374$

01_c05

MM̄FF Geometry

O - $0.458805-0.656985-0.560179$

C 1.0268050 .8217420 .747350

C $-1.1085801 .667780-0.181321$

C 0.0656631 .9237390 .415265

C $-1.5446990 .285857-0.618581$

C $0.335327-0.5522890 .623074$

C $1.362144-1.6303920 .539547$

C $2.622024-1.369498 \quad 0.171321$

C $3.052626-0.024825-0.252398$

O $2.1568430 .981981-0.129033$

H $-0.298346-0.731677 \quad 1.500507$

H 1.3880690 .9628261 .774215

C -2.783095 -0.2144470 .171615$

C $-3.181464-1.630764-0.233772$

$\begin{array}{llll}\text { O } & 4.174257 & 0.145274 & -0.713017\end{array}$

H -1.822452 $0.361822-1.677445$

O $-3.8987750 .653514-0.058440$

H $-1.7935462 .483102-0.403833$

H 0.3481482 .9418190 .664745

H $1.058091-2.6462650 .768716$

H $3.369402-2.1499150 .089904$

H -2.573056 -0.2038041 .247473$

H $-2.385983-2.351490-0.023153$

H -3.424780 -1.675865 -1.300943

H $-4.080756-1.9455540 .306824$

H $-4.653446 \quad 0.303307 \quad 0.445743$

SCF Energy $\left(B 3 L Y P / 6-31 G^{\star *}\right)=-689.142152816$ 
$01 \mathrm{c} 06$

MMFF Geometry

O $0.4653040 .646450-0.553751$

C -1.028278 -0.8141810 .762075$

C $1.115496-1.671872-0.138978$

C $-0.063711-1.9200580 .451398$

C $1.551088-0.294558-0.588347$

C $-0.3355030 .558290 \quad 0.627388$

C -1.3617331 .6350700 .523567$

C $-2.617851 \quad 1.3698050 .145641$

C $-3.0424470 .020960-0.270894$

O $-2.148150-0.984419-0.125665$

H 0.2932090 .7480051 .506232

H $-1.400395-0.9433501 .786581$

$\begin{array}{lll}\text { C } 2.784722 & 0.215846 & 0.203320\end{array}$

C $3.1645571 .644926-0.173220$

O $-4.156843-0.153910-0.746929$

H $1.837365-0.383483-1.643912$

O $3.921601-0.626362-0.022344$

H $1.802602-2.491524-0.336574$

H $-0.346764-2.9341280 .716588$

H $-1.060708 \quad 2.6529190 .748213$

H -3.365226 2.148684 0.050506

H 2.5767880 .1900931 .279051

H 2.3682602 .3533950 .072954

H $3.3836061 .721744-1.243853$

H 4.0736581 .9494020 .356726

H $4.184282-0.531201-0.954703$

SCF Energy $\left(B 3 L Y P / 6-31 G^{* *}\right)=-689.143141869$

01_c07

MM̄FF Geometry

O $-0.407495-0.688790-0.751482$

C $0.9312710 .651520 \quad 0.852848$

C $-1.228472 \quad 1.507426 \quad 0.007169$

C -0.1076921 .7188210 .712243$

C $-1.5156200 .234890-0.757639$

C $0.345318-0.7192440 .463442$

C $1.457753-1.6887230 .237396$

C $2.698420-1.274269-0.046664$

C $3.0229710 .151761-0.234605$

O 2.0495731 .0513020 .040476

H -0.284624 -1.090675 1.278507

H 1.2753630 .6259621 .895024

C $-2.827897-0.504115-0.373917$

C $-4.0695360 .372710-0.473664$

O $4.1281270 .482920-0.644489$

H -1.624355 $0.530536-1.809268$

O $-2.775910-1.0417460 .951399$

H - $1.9564262 .310392-0.075291$

H 0.0727262 .6751851 .193022

H $1.234060-2.748714 \quad 0.292815$

H $3.508529-1.969879-0.231030$

H -2.952482 -1.359202-1.050397

H $-4.1556230 .832699-1.462562$

H $-4.070046 \quad 1.1587770 .288623$

H $-4.968340-0.227394-0.292552$

H -2.272233 -1.871836 0.886224

SCF Energy $\left(B 3 L Y P / 6-31 G^{* *}\right)=-689.142533601$

01_c08

MMFF Geometry

O $0.4629710 .665688-0.581187$

C $-1.004070-0.815956 \quad 0.740514$

C $1.126420-1.652415-0.219357$

C $-0.042329-1.9128760 .386606$

C $1.561337-0.261887-0.632739$

C -0.3266490 .5639360 .605581$

C -1.3607121 .6344020 .520423$

C -2.623135 $1.363407 \quad 0.169123$

C $-3.0515550 .012596-0.235113$

O $-2.146844-0.986816-0.117653$

H $\quad 0.307720 \quad 0.753547 \quad 1.480089$

H $-1.347230-0.9617801 .772785$

C $2.7802760 .244130 \quad 0.185742$

C $3.1857651 .656499-0.223402$

O $-4.178760-0.170017-0.676614$
H $1.857751-0.323505-1.687359$

O $3.922546-0.597509-0.003135$

H $1.787802-2.473905-0.483489$

H $-0.333067-2.934650 \quad 0.612167$

H -1.0597942 .6545560 .735239$

H -3.3752002 .1394400 .088080$

H $2.5502310 .254338 \quad 1.257517$

H $2.3869912 .379117-0.032525$

H $3.4481881 .694475-1.286407$

H 4.0762101 .9728990 .330943

H $3.749007-1.4363210 .457401$

SCF Energy $\left(B 3 L Y P / 6-31 G^{* *}\right)=-689.142940005$

01_c09

MM̄FF Geometry

O $-0.886937-0.800468 \quad 0.810903$

C $1.151878 \quad 0.560748 \quad 1.071317$

C $-0.7533021 .381386-0.279354$

C 0.4214041 .6335640 .315663

C $-1.444748 \quad 0.036701-0.220482$

C $0.542630-0.8409410 .856378$

C $1.097823-1.520133-0.354549$

C $2.234194-1.100082-0.921521$

C $3.046081-0.013524-0.345649$

O 2.5526120 .6283630 .739816

H $0.806971-1.4699271 .715536$

H $\begin{array}{llll}1.071141 & 0.812738 & 2.135978\end{array}$

C $-2.965247 \quad 0.1939730 .045552$

C -3.667198 -1.1560150 .171860$

$\begin{array}{lllll}\text { O } & 4.147771 & 0.244621 & -0.813395\end{array}$

H -1.312766 - $0.454370-1.193249$

O $-3.5838870 .918202-1.024981$

H -1.245456 $2.164056-0.852981$

H 0.8720102 .6198720 .256682

H $\quad 0.558130-2.367306-0.765085$

H $2.649261-1.582500-1.798791$

H -3.121967 0.7593120 .972583

H -3.285427 -1.7356501 .017301$

H -3.543744 -1.745187-0.743481

H $-4.744628-1.0157880 .312037$

H -4.521084 $1.034944-0.790564$

SCF Energy $\left(B 3 L Y P / 6-31 G^{* *}\right)=-689.142003581$

01_c10

MMFF Geometry

O $-0.577733-0.627639-0.514880$

$\begin{array}{llll}\text { C } & 0.983535 & 0.862634 & 0.723444\end{array}$

C $-1.0764051 .786422-0.284638$

C 0.0755441 .9985390 .367526

C $-1.521850 \quad 0.431191-0.771930$

C $0.218119-0.4735550 .661415$

C $1.194728-1.6019220 .650375$

C $2.463399-1.4231840 .262252$

C $2.949723-0.128989-0.251382$

$\begin{array}{llll}\text { O } & 2.103547 & 0.925583 & -0.175944\end{array}$

H $-0.419469-0.5661461 .546535$

H 1.3681141 .0225161 .739282

C $-2.9442900 .016865-0.312411$

C $-3.112280-0.199481 \quad 1.185116$

O $4.068703-0.040531-0.739849$

H - $1.5601980 .489069-1.867636$

O $-3.315489-1.205269-0.965559$

H -1.707252 $2.633920-0.543095$

H $0.384216 \quad 3.0040910 .636871$

H $0.846868-2.5847770 .950833$

H $3.175373-2.2395970 .231655$

H $-3.6606600 .781323-0.635485$

H -2.7798360 .6657531 .765001$

H -2.579139-1.093098 1.524008

H $-4.168560-0.3782361 .417165$

H -2.513019-1.763096 -0.973876

SCF Energy (B3LYP/6-31G**)=-689.143509705

01_c11

MM̄FF Geometry

O $-0.896489-0.792972 \quad 0.814614$

C 1.1530110 .5556931 .078079 
C $-0.7531611 .396758-0.259966$

$\begin{array}{lllll}C & 0.425937 & 1.636837 & 0.331158\end{array}$

C $-1.4510260 .056603-0.205345$

C $0.533028-0.8411750 .861106$

C $1.081499-1.523318-0.351350$

C $2.217748-1.108882-0.922547$

C $3.036154-0.024622-0.351593$

$\begin{array}{lllll}\text { O } & 2.552172 & 0.616188 & 0.738424\end{array}$

H $\quad 0.793315-1.473515 \quad 1.719041$

H 1.0801480 .8036662 .144248

$\begin{array}{llll}\text { C }-2.971964 & 0.223869 & 0.057842\end{array}$

$\begin{array}{llll}\text { C }-3.685868 & -1.115011 & 0.227661\end{array}$

O $4.1340910 .232187-0.828559$

H -1.323581-0.428582 - 1.181716

O $-3.6019130 .933384-1.017334$

$\mathrm{H}-1.2467962 .192093-0.814969$

H 0.8795532 .6225130 .282427

H $\quad 0.539514-2.371483-0.756625$

H 2.629368-1.595399-1.799248

H -3.1257520 .8105790 .971514$

H -3.321756 -1.660731 1.102985

H -3.550855 -1.744672 -0.658639

H -4.764165 -0.9594360 .342363$

$\mathrm{H}-3.6040210 .354160-1.798816$

SCF Energy $\left(B 3 L Y P / 6-31 G^{* *}\right)=-689.143551681$

01_c12

MM̄FF Geometry

O $-0.891417 \quad-0.8106910 .805870$

C $1.1391240 .555943 \quad 1.072474$

C $-0.7636001 .368644-0.290676$

C 0.4083931 .6240160 .308859

C $-1.4554480 .023328-0.224351$

C $0.537282-0.8477530 .854355$

C $1.097559-1.522259-0.356623$

C $2.234699-1.097848-0.918861$

C $3.040621-0.009192-0.338444$

$\begin{array}{llllllll}\text { O } & 2.540816 & 0.629012 & 0.746558\end{array}$

H $\quad 0.802121-1.4769981 .713138$

H 1.0516880 .8097502 .136108

C -2.975586 0.1670510 .056096

C -3.668710 - 1.1916430 .117601

O $4.1414450 .256545-0.803667$

H -1.327259-0.468727 - 1.197087

O $-3.6267260 .922666-0.971052$

H $-1.2330512 .147400-0.885597$

H $\quad 0.8654592 .606970 \quad 0.239467$

H $0.562308-2.371195-0.769677$

H $2.654641-1.577962-1.795147$

H -3.139292 0.6744241 .015285

H $-3.282242-1.8094530 .933394$

H -3.544131-1.735975 - 0.825052

H $-4.746665-1.0625000 .265068$

H -3.542515 $1.862295-0.734151$

SCF Energy $\left(B 3 L Y P / 6-31 G^{\star *}\right)=-689.142982845$

01_c13

MMFF Geometry

O $0.979595 \quad 0.511698 \quad 0.805100$

C $-1.025657-0.913138 \quad 0.723528$

C $0.717794-1.084957-1.029307$

C $-0.387649-1.586435-0.459599$

C $1.4187870 .154804-0.522011$

C $-0.4343910 .483221 \quad 1.009153$

C $-1.1122841 .556995 \quad 0.220090$

C $-2.3053601 .349528-0.347655$

C $-3.0564280 .095636-0.162492$

O $-2.454134-0.8981390 .533050$

H -0.6002770 .7212142 .067184$

H $-0.833778-1.5539241 .593079$

C $2.963156-0.012537-0.510588$

C $3.468961-0.9036500 .621230$

O $-4.200983-0.003994-0.585909$

H $1.178550 \quad 0.990130-1.191891$

O $3.5820081 .277117-0.399982$

H $1.130284-1.570601-1.910146$

H $-0.853418-2.487476-0.847963$
H $-0.6115902 .514960 \quad 0.120262$

H -2.808264 $2.119695-0.920728$

H $3.291418-0.427669-1.470952$

H $3.011722-1.8967600 .589190$

H $3.273828-0.4534821 .600390$

H $4.555555-1.0251580 .549984$

H $4.5325121 .138823-0.250579$

SCF Energy $\left(B 3 L Y P / 6-31 G^{* *}\right)=-689.140011589$

01_c14

MM̄FF Geometry

O $-0.8215850 .804531-0.982972$

C $1.023582-0.826582-0.813487$

C $-0.885746-0.9283860 .757184$

C $0.227020-1.4944730 .268810$

C $-1.440405 \quad 0.3854640 .253169$

C $0.5948770 .635303-1.054007$

C $1.2883371 .586688-0.130887$

C $2.398941 \quad 1.2282920 .522728$

C $3.048290-0.0775800 .311269$

O $2.426166-0.960300-0.505431$

H $\quad 0.8898570 .920221-2.071640$

H $0.850263-1.396116-1.734854$

C $-2.975994 \quad 0.368128 \quad 0.008181$

C $-3.7886590 .246553 \quad 1.291585$

$\begin{array}{llll}\text { O } & 4.135822 & -0.312143 & 0.822737\end{array}$

H -1.229824 1.1588121 .002637

O $-3.344592-0.706629-0.865827$

H $-1.427282-1.4348451 .550716$

H $0.569363-2.4554960 .640577$

H $0.8732952 .581544-0.008246$

H 2.9152921 .9071091 .191273

H -3.252065 $1.297479-0.505355$

H -3.525260 1.0321992 .005917

H -3.649601 -0.7293091 .767463$

H -4.8583840 .3280411 .068795$

H -4.263606 -0.553758 -1.142622

SCF Energy $\left(B 3 L Y P / 6-31 G^{\star *}\right)=-689.138249322$

01_c15

MMFF Geometry

O $-0.646136 \quad 1.132794-0.258633$

C $1.118910-0.452372-1.201278$

C $-1.179762-1.159363-0.768743$

C $-0.046767-1.357844-1.458630$

C $-1.238113-0.068397 \quad 0.271866$

C $0.627400 \quad 0.984575-0.899004$

C $1.6245151 .735332-0.084661$

C 2.6117841 .1186210 .572796

C $2.739075-0.3463200 .609852$

O $1.862859-1.064377-0.126676$

H $\quad 0.5219021 .502156-1.861144$

H $1.776262-0.439900-2.079485$

C -2.669469 0.3170200 .724863

C $-3.425590-0.8094401 .413177$

O $3.586047-0.8726621 .319571$

H $-0.668951-0.375857 \quad 1.158588$

O $-3.4432440 .775352-0.391580$

H -2.030331-1.818089-0.910328

H $\quad 0.052942-2.172845-2.166799$

H $1.5296222 .815875-0.038296$

H 3.3332631 .6679911 .166777

H -2.599030 1.1672021 .414377

H -2.860395 -1.207837 2.260958

H -3.655731-1.626900 0.722829

H - $-4.389923-0.4427611 .782276$

H -2.857721 $1.391235-0.872641$

SCF Energy $\left(B 3 L Y P / 6-31 G^{\star *}\right)=-689.139191515$

$01 \_c 16$

MMFF Geometry

O $-0.557660-0.608790-0.546697$

C $1.0127290 .817304 \quad 0.744395$

C $-1.0507821 .784038-0.218597$

C 0.1080901 .9685830 .430030

C - $1.5196720 .443582-0.737692$

C $0.248789-0.515408 \quad 0.624658$ 
C $1.224026-1.6425330 .549156$

C $2.493148-1.4472050 .171763$

C $2.985054-0.128339-0.266179$

O $2.1401210 .922194-0.142353$

H $-0.377223-0.652349 \quad 1.512080$

H $1.3909830 .935097 \quad 1.768318$

C $-2.9445570 .061702-0.241833$

C $-3.028158-0.3754061 .214365$

O $4.110002-0.014598-0.736031$

H $-1.5921290 .542518-1.828722$

O $-3.473482-0.991125-1.058825$

H $-1.6736302 .644690-0.450707$

H 0.4283172 .9640110 .721976

H $\quad 0.869301-2.640148 \quad 0.786243$

H $3.200176-2.2643950 .092210$

H $-3.6063310 .924511-0.387460$

H -2.592676 0.3672391 .888580

H -2.538447 -1.3423901 .367754$

H $-4.075595-0.5204681 .502690$

H $-4.356598-1.217681-0.722124$

SCF Energy $\left(B 3 L Y P / 6-31 G^{* *}\right)=-689.137535417$

01_c17

MMFF Geometry

O $0.683512-0.672901-0.922624$

C -1.232965 $1.011379-0.815264$

C $1.0647501 .617384-0.246386$

C $-0.1465262 .028066-0.648331$

C $1.307697 \quad 0.1593910 .063770$

C $-0.650077-0.333058-1.311487$

C $-1.526597-1.476111-0.928406$

C $-2.472643-1.3617240 .009023$

C $-2.668300-0.1275830 .784738$

$\begin{array}{llll}0 & -1.902908 & 0.937405 & 0.460890\end{array}$

H - $-0.621026-0.287859-2.407887$

H $-1.969667 \quad 1.380215-1.539868$

C $2.820309-0.1818740 .103221$

C $3.061916-1.6656420 .371900$

O $-3.474627-0.1018571 .705504$

H $0.873547-0.0627321 .047744$

$\begin{array}{lllll}\text { O } & 3.476897 & 0.571444 & 1.129322\end{array}$

H $1.8529282 .336256-0.041686$

H - $-0.3692563 .079026-0.794208$

H - $1.374168-2.422646-1.437556$

H -3.104312 -2.197071 0.288366

H $3.2830250 .079654-0.856501$

H $2.635513-2.296076-0.413777$

H $2.629689-1.9615251 .334106$

H $4.135697-1.8740630 .432722$

H $4.4196520 .332726 \quad 1.107137$

SCF Energy $\left(B 3 L Y P / 6-31 G^{* *}\right)=-689.134517910$

01_c18

MMFF Geometry

O $0.730439-0.617949-1.006386$

C -1.232656 $1.000824-0.849089$

C $1.0563141 .631076-0.246275$

C $-0.1555902 .027820-0.663213$

C 1.3045130 .1685180 .044519

C $-0.636955-0.345591-1.330885$

C $-1.448842-1.509249-0.878012$

C $-2.388604-1.3988320 .065435$

C -2.644899 -0.1418150 .781994$

$\begin{array}{lllll} & -1.925200 & 0.940476 & 0.416754\end{array}$

H $-0.663178-0.333440-2.428210$

H $-1.9585781 .362182-1.587876$

C $2.815496-0.1694020 .150171$

C $3.051533-1.6702950 .299179$

O $-3.457996-0.110187 \quad 1.696511$

H $0.824305-0.0881320 .998348$

$\begin{array}{lllll}\text { O } & 3.413675 & 0.457608 & 1.290565\end{array}$

H $1.8278752 .366490-0.049273$

H $-0.3899193 .075886-0.814078$

H - $1.255976-2.470504-1.344675$

H -2.974065 -2.2515690 .389903$

H $3.3477540 .171363-0.746513$

H $2.702568-2.226366-0.575827$
H $2.542494-2.0583951 .188403$

H $4.119177-1.8765690 .433502$

H $3.4683501 .411155 \quad 1.113801$

SCF Energy $\left(B 3 L Y P / 6-31 G^{* *}\right)=-689.134209596$

02 c01

MMFF Geometry

O $0.464832-0.677368-0.538854$

$\begin{array}{llll}\text { C }-1.005468 & 0.834928 & 0.750259\end{array}$

C $1.1441911 .650566-0.182912$

$\begin{array}{llll}\text { C } & -0.027430 & 1.921264 & 0.412474\end{array}$

C $1.5618130 .264588-0.607598$

C $-0.334601-0.5498710 .640778$

C $-1.377102-1.6129250 .560406$

C $-2.630415-1.3361710 .181227$

C $-3.036540 \quad 0.010720-0.259170$

O $-2.1257341 .004398-0.136940$

H $0.290239-0.731437 \quad 1.523619$

H -1.3717210 .9906821 .773170$

C $2.766181-0.3149440 .177536$

C $4.0454200 .492448 \quad 0.009838$

$\begin{array}{llll}\text { O } & -4.150172 & 0.194423 & -0.733234\end{array}$

H $1.8290220 .298379-1.670864$

O $3.029401-1.650197-0.277722$

H $1.8199792 .467922-0.422982$

H $-0.3043482 .944574 \quad 0.648487$

H -1.092287 -2.630775 0.805945

H -3.390059-2.105273 0.104329

H $2.526806-0.393887 \quad 1.243454$

H 3.9485691 .5001620 .423333

H $4.3218360 .570398-1.047382$

H $4.879645-0.006866 \quad 0.514926$

H $2.159420-2.037536-0.499054$

SCF Energy $\left(B 3 L Y P / 6-31 G^{* *}\right)=-689.147191954$

02_c02

MMFF Geometry

O $-1.013593 \quad 0.519548 \quad 0.759924$

C $1.020901-0.8769230 .767354$

C $-0.715731-1.174277-0.975099$

C $0.393494-1.626693-0.372872$

C $-1.4261750 .090742-0.556443$

C $0.4021010 .521753 \quad 0.978163$

C $1.066918 \quad 1.5694250 .144125$

C $2.264171 \quad 1.353688-0.411789$

C $3.0340330 .120623-0.170996$

O $2.448355-0.8461190 .574082$

H 0.5527100 .8137862 .024885

H $0.842091-1.471406 \quad 1.671918$

C $-2.965053-0.097718-0.521307$

C $-3.693587 \quad 1.216813-0.261398$

$\begin{array}{llll}\text { O } & 4.178980 & 0.019394 & -0.592404\end{array}$

H $-1.1894600 .879770-1.280920$

O $-3.331805-1.0082380 .523176$

H $-1.134896-1.731895-1.809347$

H $0.859430-2.553689-0.694672$

H 0.5585052 .5178300 .003624

H $2.7593072 .105141-1.015990$

H $-3.319297-0.519630-1.468629$

H $-3.4600251 .958571-1.031008$

H -3.4349001 .6316440 .718780$

H $-4.7768391 .053492-0.253138$

H $-2.798548-0.7448921 .297841$

SCF Energy (B3LYP/6-31G**) $=-689.144630964$

02 c03

MMFF Geometry

O $0.549653-0.568476-0.706120$

C $-0.934138 \quad 0.827547 \quad 0.692416$

C $1.0669581 .806166-0.393788$

C $-0.0765541 .988746 \quad 0.283604$

C $1.5592310 .448162-0.839732$

C $-0.174800-0.5054920 .523955$

C -1.142318 -1.6418190 .498787$

C -2.437055-1.457635 0.217052

C $-2.973258-0.141333-0.169460$

O $-2.1334420 .916791-0.101812$ 
H $\quad 0.507469-0.6545451 .366764$ H -1.2297130 .9550881 .741738$ C $2.905440 \quad 0.045199-0.174574$ C $3.377095-1.328165-0.641053$ O $-4.130726-0.037950-0.556022$ H $1.7430670 .516946-1.919410$ $\begin{array}{llll}\text { O } & 2.805984 & 0.022420 & 1.254965\end{array}$ H $1.6723932 .666221-0.665736$ H -0.4097642 .9866030 .550171$ H $-0.761193-2.6363130 .705150$ H -3.139429 -2.281785 0.182821 H $3.6640720 .795263-0.429059$

H $3.460857-1.370999-1.731092$

H $2.695405-2.117255-0.305843$

H $4.356819-1.560961-0.209657$

H $3.684676-0.2088051 .602807$

SCF Energy (B3LYP/6-31G**)= -689.143286693

02_c04

MMFF Geometry

O $0.553259-0.594166-0.717620$

C $\begin{array}{lllll}0.906506 & 0.826551 & 0.675442\end{array}$

C $1.0960961 .773922-0.446321$

C $-0.0387541 .973965 \quad 0.241325$

C $1.5785070 .402791-0.864344$

C $-0.166248-0.5181580 .513500$

C $-1.146825-1.6423160 .499015$

C $-2.441566-1.444600 \quad 0.226746$

C $-2.966433-0.123863-0.159447$

O $-2.1126690 .924195-0.106231$

H $0.518709-0.6698961 .353839$

$\mathrm{H}-1.1881210 .970367 \quad 1.726418$

C $2.9118940 .005448-0.169452$

C $3.349788-1.405468-0.542383$

O $-4.126655-0.006786-0.532983$

H $1.7754020 .444113-1.942869$

$\begin{array}{lllll}\text { O } & 2.822929 & 0.060381 & 1.260334\end{array}$

H $1.6944572 .626535-0.757161$

H -0.3724192 .9792500 .480589$

H $-0.775491-2.6408950 .705182$

H $-3.153093-2.261327 \quad 0.200733$

H $3.6982470 .710320-0.465973$

H $3.444166-1.519465-1.626408$

H $2.644147-2.154421-0.166942$

H $4.317699-1.635278-0.083413$

H $2.6816700 .992071 \quad 1.503520$

SCF Energy (B3LYP/6-31G**)= -689.146074286

\section{2 c05}

MMFF Geometry

O $0.895250-0.8322660 .776863$

C -1.1349910 .5305001 .086265$

C $0.7738541 .392747-0.241303$

C $-0.3991821 .624596 \quad 0.365805$

C $1.4595230 .048821-0.227942$

C $-0.535025-0.8659940 .821604$

C $-1.093064-1.497913-0.413047$

C $-2.226998-1.051575-0.964714$

C $-3.0318950 .019813-0.351643$

$\begin{array}{llll}\text { O } & -2.534971 & 0.618299 & 0.756931\end{array}$

H $-0.802891-1.5238571 .657692$

H -1.0521350 .7455432 .158787$

$\begin{array}{llll}\text { C } 2.974670 & 0.123587 & 0.091098\end{array}$

C $3.7908740 .869784-0.954278$

O $-4.1290230 .305753-0.813356$

H $1.332293-0.437122-1.203238$

O $3.498636-1.2115430 .173036$

H $1.2465472 .202260-0.790939$

$\begin{array}{llll}\mathrm{H} & -0.851048 & 2.612313 & 0.340275\end{array}$

H $-0.559987-2.334944-0.852629$

H -2.645661 - $1.501363-1.857634$

H $3.1298740 .578215 \quad 1.076837$

H $3.5289271 .930043-1.000372$

H $3.6494830 .428710-1.947088$

H $4.8601690 .792855-0.727891$

H $2.858687-1.7007940 .727337$

SCF Energy $\left(B 3 L Y P / 6-31 G^{* *}\right)=-689.146261472$ 02_c06

MMFF Geometry

O $0.579073-0.586971-0.544776$

C -1.0028210 .8513650 .718372$

C $1.0525191 .816281-0.256423$

C -0.1150882 .0059330 .374668$

C $1.5383650 .468640-0.738442$

C $-0.215913-0.471466 \quad 0.637317$

C $-1.173244-1.6164700 .605299$

C $-2.444573-1.453073 \quad 0.219563$

C $-2.952583-0.159304-0.272918$

O $-2.1259610 .908590-0.179042$

H $\quad 0.422490-0.571327 \quad 1.521455$

H $-1.3887680 .991617 \quad 1.736519$

C $2.9331300 .084352-0.174664$

C $3.503386-1.149853-0.870294$

O $-4.072268-0.083532-0.762440$

H $1.6478280 .557716-1.827017$

O $2.889189-0.1722331 .233443$

H $1.6791042 .674720-0.485611$

H -0.4416553 .0037310 .650132$

H $-0.808828-2.5981510 .888475$

H -3.142213 - 2.2809530 .174352

H $3.6267060 .920287-0.322791$

H $3.605434-0.985981-1.947295$

H $2.869930-2.028688-0.708218$

H $4.489247-1.394692-0.460278$

H $2.541396-1.0735461 .347927$

SCF Energy $\left(B 3 L Y P / 6-31 G^{\star *}\right)=-689.143184365$

02_c07

MMFF Geometry

O -0.8386020 .7816750 .983349$

C $1.003268-0.8488710 .787812$

C $-0.889183-0.892000-0.805789$

C $0.208635-1.485404-0.316266$

C $-1.4294440 .419051-0.280420$

C $0.579407 \quad 0.6092581 .065291$

C $1.288143 \quad 1.5818740 .177596$

C $2.4034451 .234953-0.474429$

C $3.044002-0.079730-0.292884$

$\begin{array}{llll}\text { O } & 2.406585 & -0.983475 & 0.488805\end{array}$

H $\quad 0.8624900 .8632362 .094387$

H $0.818886-1.4408201 .692843$

C $-2.970087 \quad 0.414687-0.096540$

C $-3.470702-0.6708420 .853551$

O $4.138131-0.304895-0.794177$

H $-1.1864881 .196045-1.016740$

O $-3.6227750 .271698-1.362325$

H $-1.430524-1.350808-1.630509$

H $0.549170-2.436573-0.714501$

H 0.8785582 .5814290 .074623

H $2.9290361 .928714-1.120213$

H $-3.267318 \quad 1.387520 \quad 0.314477$

H $-2.988526-0.6040631 .833193$

H $-3.302588-1.6715170 .441841$

H $-4.552880-0.5763960 .996821$

H -4.581432 $0.305349-1.198974$

SCF Energy $\left(B 3 L Y P / 6-31 G^{* *}\right)=-689.142036721$

02_c08

MMFF Geometry

O -0.8466490 .7155291 .034158$

C $1.000937-0.8973450 .742499$

C $-0.897653-0.856991-0.845180$

C $0.204689-1.471785-0.393940$

C $-1.4398130 .418530-0.243333$

C $0.5716540 .539965 \quad 1.107296$

C $1.276644 \quad 1.5678820 .280884$

C $2.3908441 .265281-0.394574$

C $3.033939-0.057278-0.299873$

O $2.403311-1.0079910 .429388$

H 0.8533410 .7321962 .150071

H $0.823371-1.5423761 .611908$

C -2.981411 $0.401479-0.059720$

C $-3.481711-0.750693 \quad 0.806559$ 
O $4.123727-0.249171-0.823704$

H -1.200283 $1.235957-0.935717$

$\begin{array}{llll}\text { O } & -3.653631 & 0.336928 & -1.321923\end{array}$

H -1.444513 -1.279611-1.685669

H $0.544330-2.400794-0.842562$

H 0.8682122 .5725440 .246500

H $2.9153552 .000187-0.994177$

H -3.2880551 .3359190 .426807$

H $-3.005067-0.7519691 .791210$

H $-3.307838-1.7194330 .326414$

H $-4.565280-0.670098 \quad 0.948350$

H -3.476342 1.170857-1.790192

SCF Energy $\left(B 3 L Y P / 6-31 G^{* *}\right)=-689.143440415$

02_c09

MMFF Geometry

$\begin{array}{lllll}0 & -0.842935 & 0.859800 & 0.917688\end{array}$

C $0.988639-0.7876200 .848286$

C $-0.896885-0.932485-0.751396$

C $0.195203-1.494795-0.213973$

C $-1.4344320 .414314-0.318045$

C $0.573260 \quad 0.6887001 .018408$

C 1.2903581 .5906610 .065554

C $2.4062591 .191789-0.554996$

C $3.037638-0.110559-0.277614$

O $2.391980-0.9517160 .565069$

H $0.853611 \quad 1.0146892 .027742$

H $0.796140-1.3134901 .791569$

C $-2.9759040 .430262-0.132143$

C $-3.482455-0.5459520 .927962$

O $4.129955-0.380013-0.760399$

H $-1.1889501 .137176-1.106709$

O $-3.6499270 .187331-1.371476$

$\mathrm{H}-1.416448-1.444809-1.556551$

H $\quad 0.540290-2.469159-0.547702$

H $0.8875572 .583096-0.109459$

H 2.938808 1.834966 -1.245961

H $-3.264851 \quad 1.4390420 .187181$

H -3.023112 -0.3565621 .902549$

H -3.287281-1.585164 0.643138

H -4.569021 -0.4541391 .034205$

H -3.709420 -0.774767-1.499305

SCF Energy $\left(B 3 L Y P / 6-31 G^{* *}\right)=-689.143214093$

\section{2 c10}

MMFF Geometry

O $-0.403456 \quad 0.725310-0.709354$

C $0.959502-0.6313240 .869294$

C $-1.209220-1.4823300 .050261$

C $-0.089555-1.6919520 .756921$

C $-1.483399-0.223446-0.741485$

$\begin{array}{llll}\text { C } & 0.372524 & 0.741926 & 0.488422\end{array}$

C $1.486767 \quad 1.7051940 .246490$

C $2.7202841 .283997-0.058289$

C $3.034507-0.143964-0.251211$

$\begin{array}{llll}\text { O } & 2.060439-1.038496 & 0.038707\end{array}$

H -0.2421691 .1137531 .314359$

H $1.323277-0.6032391 .904839$

C -2.845534 $0.455237-0.428299$

C $-3.025340 \quad 0.917407 \quad 1.012861$

O $4.132606-0.480975-0.675042$

H -1.542662 - $0.547612-1.789230$

O $-3.917550-0.440066-0.752284$

H $-1.956353-2.269884-0.024504$

H $0.083371-2.6422551 .252043$

H 1.2682562 .7662640 .304006

H $3.5306901 .975407-0.256595$

H $-2.9474631 .331387-1.080549$

H -2.3314931 .7215631 .268009$

H -2.8931680 .0916561 .719230$

H -4.0423871 .2954431 .167094$

$\mathrm{H}-4.7494270 .056855-0.664706$

SCF Energy $\left(B 3 L Y P / 6-31 G^{* *}\right)=-689.139868259$

02_c11

MMFF Geometry

O $-0.4051350 .725056-0.703624$
C $0.956291-0.6218910 .880655$

C $-1.221868-1.469520 \quad 0.079206$

C $-0.098830-1.678646 \quad 0.780938$

C $-1.492064-0.213345-0.716465$

C $0.374056 \quad 0.751568 \quad 0.492458$

C $1.4918941 .707151 \quad 0.237650$

C $2.7211321 .277231-0.072294$

C $3.025069-0.153582-0.260275$

$\begin{array}{llll}\text { O } & 2.048427 & -1.040712 & 0.043939\end{array}$

H -0.2367251 .1335561 .316518$

H $1.327340-0.5886281 .913400$

C -2.847076 $0.478206-0.399028$

C $-3.0403300 .893008 \quad 1.054038$

O $4.115995-0.499831-0.694869$

H - $1.560696-0.544007-1.761537$

O $-3.943062-0.378724-0.747104$

H -1.974974 -2.252819 0.024049

H $\quad 0.071287-2.6241411 .286456$

H 1.2798922 .7697930 .292039

H $3.5344091 .962843-0.278939$

H $-2.934807 \quad 1.381947-1.014603$

H -2.3380031 .6758721 .348482$

H -2.934523 0.0420721 .734534

H -4.054259 1.2817231 .203422

H -3.918390 -0.505607-1.711853

SCF Energy $\left(B 3 L Y P / 6-31 G^{* *}\right)=-689.141598766$

02_c12

MMFF Geometry

O $-0.402556 \quad 0.720546-0.741116$

C $0.935783-0.6245150 .863845$

C $-1.225666-1.4770210 .014937$

C $-0.113179-1.6849240 .733949$

C -1.496792 $-0.210402-0.767909$

C 0.3623690 .7484090 .463314

C 1.4829381 .7039420 .222177

C $2.7195531 .275563-0.058670$

C $3.033053-0.155431-0.225695$

O $2.050452-1.0428290 .057223$

H -0.2569861 .1318361 .279989$

H $1.279514-0.5925231 .906008$

C -2.848412 $0.483139-0.435422$

C -3.001612 0.9802830 .997378

O $4.137575-0.502394-0.623697$

H - $1.572052-0.528831-1.816285$

O $-3.949701-0.385526-0.734066$

H - $1.950203-2.281233-0.086427$

H $0.064986-2.641166 \quad 1.216437$

H 1.2666622 .7665220 .260136

H $3.5342531 .962398-0.255617$

H $-2.9520061 .352348-1.096013$

H -2.319254 1.8060311 .211996

H -2.8310020 .1779591 .722283$

H -4.0232381 .3393341 .166046$

H $-4.015049-1.045321-0.022733$

SCF Energy $\left(B 3 L Y P / 6-31 G^{* *}\right)=-689.140608205$

02 c13

MMFF Geometry

O $-0.981044 \quad 0.639068 \quad 0.714184$

C $1.006599-0.8237880 .804708$

C $-0.716809-1.160545-0.937302$

C $0.385414-1.604377-0.317022$

C $-1.4286140 .116384-0.551562$

C $0.429750 \quad 0.6014470 .933328$

C $1.128844 \quad 1.576571 \quad 0.039714$

C $2.3270201 .296395-0.484589$

C $3.0621840 .063702-0.150591$

O $2.439502-0.8405990 .641652$

H $0.5928460 .950201 \quad 1.960711$

H $0.791178-1.3706431 .730940$

C $-2.970449-0.077252-0.500812$

C $-3.6926001 .216275-0.133648$

O $4.211995-0.090325-0.542486$

H $-1.2135600 .869586-1.320378$

O $-3.328970-1.0858910 .452468$

H -1.139300 -1.740795 -1.753304 
H $0.844682-2.544847-0.605809$

H $0.6436522 .524309-0.168807$

H $2.8478931 .993974-1.129908$

H -3.323971-0.419800 -1.480746

H -3.430042 $2.026545-0.820401$

H $-3.458193 \quad 1.5275610 .889916$

H $-4.777643 \quad 1.069744-0.171859$

H -4.297432 -1.092741 0.529750

SCF Energy $\left(B 3 L Y P / 6-31 G^{* \star}\right)=-689.138669051$

02 c14

MMFF Geometry

O $0.434463-0.684709-0.590284$

C -1.0099780 .8080720 .736438$

C $1.1238691 .623512-0.238239$

C $-0.040504 \quad 1.8949990 .371700$

C $1.5515460 .229300-0.640664$

C $-0.350861-0.5797350 .595927$

C $-1.397836-1.637598 \quad 0.508218$

C $-2.659749-1.351745 \quad 0.167918$

C $-3.0777370 .006351-0.220691$

O $-2.1612950 .995223-0.107436$

H $0.279121-0.7801691 .470677$

H -1.3398900 .9581591 .772422$

C $2.761546-0.3193700 .170639$

C $3.9831900 .593611 \quad 0.121776$

$\begin{array}{lllll}\text { O } & -4.208856 & 0.205084 & -0.645507\end{array}$

H $1.8380160 .255678-1.699218$

O $3.151644-1.594562-0.357230$

H $1.7868152 .442221-0.506486$

H -0.3226322 .9200740 .592729$

H $-1.102655-2.6625520 .707695$

H $-3.418613-2.1205640 .083530$

H $2.474208-0.486611 \quad 1.214079$

H $3.794037 \quad 1.5558540 .605657$

H $4.2938760 .773016-0.913347$

H 4.8340280 .1227090 .626597

H $3.855117-1.9504820 .211188$

SCF Energy $\left(B 3 L Y P / 6-31 G^{* *}\right)=-689.139005681$

$02 \_15$

MMFF Geometry

O $0.829782-0.453813 \quad 0.755856$

C $-1.152218 \quad 1.1500390 .614394$

C $1.0208691 .673995-0.370004$

C -0.1369432 .1365290 .123543$

C $1.254650 \quad 0.186842-0.459047$

C $-0.450670-0.0656931 .267962$

C $-1.324777-1.272355 \quad 1.232744$

C $-2.386969-1.3545830 .425005$

C $-2.722991-0.299993-0.544003$

O $-1.9657360 .819208-0.530773$

H -0.2702220 .1885562 .320343$

H $-1.810598 \quad 1.6335151 .346773$

C $2.749149-0.159700-0.675412$

C $2.961010-1.653438-0.898212$

O $-3.630443-0.469070-1.347767$

H $0.675314-0.219414-1.298140$

$\begin{array}{lllll}\text { O } & 3.517651 & 0.219597 & 0.474516\end{array}$

H $1.7646512 .355371-0.772465$

H -0.3664723 .1960960 .138982$

H - $-1.074425-2.0984831 .891157$

H -3.018240 -2.235440 0.398634

H $3.1467490 .389495-1.536331$

H $2.402264-2.007554-1.769684$

H $2.655959-2.236570-0.022600$

H $4.024093-1.865016-1.057060$

H $2.994251-0.0778021 .243932$

SCF Energy $\left(B 3 L Y P / 6-31 G^{* *}\right)=-689.138531373$

$02 \_c 16$

MMFF Geometry

O $0.856353-0.7935840 .872167$

C - -1.1595330 .5987341 .055866$

C $0.7750351 .341404-0.306922$

C $-0.401708 \quad 1.6303290 .268537$

C $1.446728-0.009057-0.192537$
C $-0.571356-0.8179090 .900306$

C $-1.122032-1.532984-0.291431$

C $-2.244412-1.117997-0.888801$

C $-3.0442280 .003396-0.365813$

$\begin{array}{llll}\text { O } & -2.554332 & 0.677842 & 0.701621\end{array}$

H $-0.854984-1.4106391 .778840$

H -1.0883730 .8900172 .111103$

$\begin{array}{llll}\text { C } & 2.972190 & 0.050038 & 0.103337\end{array}$

C $3.7624860 .969134-0.820399$

O $-4.132710 \quad 0.266140-0.861197$

H $1.303096-0.557501-1.132140$

O $3.524426-1.268959-0.054333$

H $1.2526132 .101386-0.918559$

H $-0.8438402 .616834 \quad 0.162435$

H $-0.587959-2.402961-0.660559$

H $-2.655688-1.627907-1.752104$

H $3.1318660 .339498 \quad 1.149230$

H $3.5149572 .021950-0.660328$

H $3.5799250 .717133-1.870787$

H $4.8385270 .848411-0.651888$

H $4.380940-1.2782590 .405822$

SCF Energy (B3LYP/6-31G** $)=-689.137950620$

02_c17

MMFF Geometry

O $-0.695981-0.708275-0.914663$

C $1.2130810 .985324-0.864566$

C $-1.0932231 .605386-0.336069$

C $0.1166942 .001340-0.758062$

$\begin{array}{llll}\text { C }-1.314219 & 0.166713 & 0.051964\end{array}$

C $0.643213-0.384352-1.305581$

C $1.518702-1.503572-0.856322$

C $2.450897-1.3399010 .087903$

C $2.629359-0.069797 \quad 0.807925$

$\begin{array}{lllll}\text { O } & 1.861322 & 0.973785 & 0.424555\end{array}$

H $0.626601-0.391629-2.403038$

H $1.9586501 .325300-1.594065$

C $-2.804613-0.2499880 .111389$

C -3.5841250 .4323551 .226276$

$\begin{array}{lllll}\text { O } & 3.420793 & 0.004967 & 1.738548\end{array}$

H - $-0.852387-0.0380241 .026503$

O $-2.888185-1.6670320 .329688$

H - $-1.8850372 .334302-0.199082$

H $0.3313013 .042022-0.974917$

H $1.380466-2.473983-1.323098$

H $3.083004-2.1576910 .414806$

H $-3.288445-0.056709-0.853430$

H -3.6413501 .5149431 .086341$

H -3.1282200 .2283252 .201409$

H $-4.6056590 .038787 \quad 1.271361$

H -2.217128 - $2.054260-0.266959$

SCF Energy $\left(B 3 L Y P / 6-31 G^{* *}\right)=-689.138803309$

$02 \_18$

MMFF Geometry

O $0.637721 \quad 1.108191-0.287642$

C $-1.107544-0.541148-1.150676$

C $1.174517-1.232300-0.603710$

C $0.051381-1.479462-1.293384$

C $1.245295-0.0389440 .320801$

C $-0.6134270 .910683-0.950901$

C $-1.629218 \quad 1.732105-0.233401$

C -2.6447501 .1788950 .437253$

C $-2.792489-0.277141 \quad 0.582839$

O $-1.896983-1.058952-0.059518$

H $-0.475704 \quad 1.346323-1.948974$

H $-1.733646-0.594597-2.050087$

C 2.6869440 .3577590 .739495

C $3.6069420 .690968-0.432872$

$\begin{array}{llll}\text { O } & -3.677299 & -0.738676 & 1.291627\end{array}$

H $\quad 0.691336-0.285861 \quad 1.236400$

O $3.295728-0.652693 \quad 1.549434$

H $1.994729-1.940852-0.635669$

H $-0.058213-2.371259-1.900623$

H - $-1.5213462 .811872-0.266966$

H $-3.377874 \quad 1.7804030 .962317$

H $2.618124 \quad 1.256422 \quad 1.364753$ 
H $3.1939081 .490604-1.054700$

H $3.790862-0.185885-1.062262$

H $4.5860581 .013101-0.061350$

H $3.552512-1.3917250 .973291$

SCF Energy $\left(B 3 L Y P / 6-31 G^{\star *}\right)=-689.134646423$

03 c01

MMFF Geometry

O $-0.483966-0.915943-0.431238$

C $1.0474610 .980543-0.759817$

C $-1.3961391 .339468-0.500404$

C $-0.1549851 .850932-0.537272$

C $-1.687178-0.140211-0.632446$

C $0.613134-0.430099-1.208779$

C $1.733287-1.394322-1.016343$

C $2.745181-1.133481-0.180306$

C 2.7723110 .0746320 .663871

$\begin{array}{lllll}O & 1.790371 & 0.986314 & 0.473046\end{array}$

H $0.323599-0.417455-2.267797$

H $1.6835851 .438092-1.528252$

C $-2.719880-0.6834270 .389215$

C $-4.108021-0.0766890 .250605$

$\begin{array}{lllll}\text { O } & 3.641275 & 0.211782 & 1.515061\end{array}$

H $-2.057459-0.340486-1.645760$

O $-2.264285-0.473891 \quad 1.730949$

H $-2.2372792 .012050-0.356524$

H $0.0072912 .915854-0.399370$

H $1.704239-2.329499-1.565817$

H $3.556076-1.835215-0.023739$

H -2.795675 -1.770099 0.260862

H $-4.488963-0.182089-0.769479$

H $-4.1179700 .981943 \quad 0.528555$

H $-4.805000-0.5759850 .932981$

$\mathrm{H}-1.310641-0.6873021 .721861$

SCF Energy $\left(B 3 L Y P / 6-31 G^{\star \star}\right)=-689.147463331$

$03 \_02$

MMFF Geometry

O $-0.675310-0.619184-0.562384$

C $1.0951401 .092346-0.643605$

C $-1.277358 \quad 1.744473-0.329920$

C $0.0198922 .088458-0.318154$

C $-1.7539460 .340133-0.613832$

C $0.483345-0.177528-1.272950$

C $1.468153-1.295063-1.214102$

C $2.484762-1.284701-0.343617$

C $2.644403-0.217796 \quad 0.661373$

$\begin{array}{lllll}0 & 1.802913 & 0.839706 & 0.583721\end{array}$

H $\quad 0.2101000 .012868-2.319079$

H $1.8052311 .550355-1.344109$

C $-2.846290-0.1428840 .371725$

C $-2.371796-0.2464321 .818634$

O $3.495805-0.317157 \quad 1.535420$

H -2.167338 $0.303758-1.629912$

O $-3.294845-1.443959-0.034782$

H -2.033152 $2.497052-0.118388$

H $0.3228023 .102016-0.073033$

H $1.333514-2.133185-1.889880$

H $3.195827-2.100140-0.281503$

H -3.7129880 .5259200 .323409$

H -2.055724 0.7238232 .212191

H - $1.541420-0.9538591 .917800$

H -3.179729 -0.6276342 .452996$

H -2.483450-1.982258 -0.119601

SCF Energy $\left(B 3 L Y P / 6-31 G^{\star *}\right)=-689.147476991$

03_c03

MMFF Geometry

O $-0.509367-0.732589-0.347591$

C $1.1759181 .034785-0.655440$

C $-1.2088391 .599808-0.282930$

C $0.0698322 .002514-0.351425$

C $-1.636846 \quad 0.155577-0.451308$

C $0.595750-0.308241-1.147620$

C $1.630750-1.376295-1.045751$

C $2.687953-1.250033-0.235049$

C $2.850374-0.094591 \quad 0.665792$
$\begin{array}{llll}O & 1.959597 & 0.917229 & 0.546452\end{array}$

H $0.268627-0.218584-2.191905$

H $1.825025 \quad 1.465758-1.428449$

C $-2.687944-0.2508540 .612657$

C $-3.064588-1.7268350 .512989$

O $3.749690-0.0839691 .496687$

H -2.080549 $0.047955-1.449835$

$\begin{array}{lllll}\mathrm{O} & -3.882466 & 0.524124 & 0.447227\end{array}$

H $-1.9945712 .327777-0.092645$

H $0.3311593 .043854-0.190899$

H $1.498270-2.275173-1.638600$

H $3.435803-2.028614-0.141776$

H $-2.292765-0.0559541 .617371$

H $-2.205860-2.3790250 .696824$

H -3.476340 $-1.956901-0.475814$

H $-3.843775-1.9732091 .242601$

H $-4.478817 \quad 0.2887501 .179443$

SCF Energy $\left(B 3 L Y P / 6-31 G^{* *}\right)=-689.144465210$

03 c04

MM̄FF Geometry

O $-0.507743-0.754959-0.334143$

C $1.164543 \quad 1.012596-0.688698$

C $-1.2258361 .573922-0.338130$

C $0.0504201 .982108-0.420259$

C $-1.643147 \quad 0.120576-0.454697$

C $0.594596-0.346747-1.144923$

C $1.637322-1.403453-1.009411$

C $2.689398-1.247087-0.197079$

C $2.838172-0.066240 \quad 0.672873$

$\begin{array}{lllll}O & 1.939803 & 0.934943 & 0.521218\end{array}$

H $\quad 0.268360-0.289017-2.191645$

H $1.8159751 .424766-1.469997$

C $-2.675462-0.276150 \quad 0.632232$

C $-3.097222-1.7372590 .502872$

O $3.732585-0.0248891 .507929$

H -2.095236 -0.020563 -1.445193

O -3.8647080 .5153730 .530346$

H -2.010412 $2.311890-0.194818$

H $0.3058843 .031186-0.305990$

H $1.514449-2.319501-1.577684$

H $3.442425-2.017278-0.078658$

H $-2.248413-0.1341191 .633169$

H -2.252891 -2.4184740 .643168$

H $-3.544225-1.928825-0.478900$

H $-3.862483-1.979678 \quad 1.248517$

H -3.729642 1.3022711 .086545

SCF Energy (B3LYP/6-31G**) $=-689.145193844$

03 c05

MM̄FF Geometry

O $0.520865-0.7239620 .344029$

C $-1.168516 \quad 1.0450840 .638669$

$\begin{array}{llll}\text { C } & 1.210727 & 1.609753 & 0.225689\end{array}$

$\begin{array}{llll}\text { C } & -0.068276 & 2.009623 & 0.304187\end{array}$

C $1.643258 \quad 0.170597 \quad 0.414114$

C $-0.579614-0.2888451 .145214$

C -1.611680-1.361799 1.070905

C -2.680168 -1.2499270 .272972$

C $-2.860495-0.106628-0.639927$

O $-1.9685390 .907143-0.549841$

H $-0.243204-0.1812872 .184866$

H -1.807734 1.4876321 .413387

C $2.691815-0.247397-0.649836$

C $3.035360-1.733426-0.581765$

O $-3.775321-0.107462-1.453646$

H 2.0979160 .0836091 .409787

$\begin{array}{lllll} & 3.904196 & 0.502230 & -0.486555\end{array}$

H $1.9886672 .336224-0.000462$

H -0.3356543 .0459950 .121759$

H -1.468443 $-2.252921 \quad 1.672884$

H -3.426337 -2.032352 0.199913

H $2.306374-0.031945-1.653648$

H $2.168024-2.360650-0.807564$

H $3.412800-2.0021060 .410989$

H $3.829892-1.973450-1.296727$

H $4.353626 \quad 0.1754460 .311588$ 
SCF Energy $\left(B 3 L Y P / 6-31 G^{\star \star}\right)=-689.145731579$

03_c06

MMFF Geometry

O $-0.650811-0.556873-0.581169$

C $1.1359101 .131134-0.544789$

C $-1.2259121 .773089-0.138901$

C $0.0751932 .102340-0.112028$

C $-1.724030 \quad 0.404688-0.553774$

C $0.502203-0.075144-1.269423$

C $1.473447-1.204985-1.316966$

C $2.504090-1.278166-0.466573$

C $2.695171-0.2979830 .617397$

$\begin{array}{lllll}\text { O } & 1.867085 & 0.772377 & 0.642177\end{array}$

H $\quad 0.2191130 .204600-2.292641$

H $1.8354661 .643759-1.217470$

C -2.845004 -0.1192560 .383049$

C -2.326997-0.608385 1.733683

O $3.562132-0.4763491 .463564$

H -2.129077 $0.475308-1.571604$

O $-3.546653-1.189075-0.267138$

H $-1.969403 \quad 2.5123250 .148145$

H $0.392143 \quad 3.085605 \quad 0.221815$

H $1.311090-1.986378-2.051993$

H $3.202054-2.106808-0.481601$

H -3.5843980 .6737130 .546451$

H $-1.765196 \quad 0.170548 \quad 2.257212$

H - $1.689417-1.4916221 .619954$

H $-3.163906-0.9135792 .371607$

H $-4.096916-1.6321040 .400609$

SCF Energy $\left(B 3 L Y P / 6-31 G^{* *}\right)=-689.142136019$

03_c07

MMFF Geometry

O $-0.464214-0.975305-0.316389$

C $1.0825030 .867411-0.865031$

C $-1.3557641 .274554-0.652856$

C $-0.1118031 .769034-0.756075$

C -1.655937-0.208565 -0.591394

C $0.640822-0.586738-1.132320$

C $1.755005-1.525622-0.817048$

C $2.766477-1.167360-0.017675$

C $2.804956 \quad 0.1400320 .661887$

$\begin{array}{lllll}\text { O } & 1.838956 & 1.033486 & 0.348017\end{array}$

H $0.359696-0.703404-2.187367$

H $1.7156291 .217616-1.690518$

C $-2.717845-0.602097 \quad 0.472873$

C $-4.123627-0.137602 \quad 0.112844$

$\begin{array}{lllll}\text { O } & 3.680278 & 0.378468 & 1.484623\end{array}$

H -2.024328 $-0.528360-1.574796$

O $-2.384915-0.066399 \quad 1.758298$

H -2.192577 $1.963124-0.581923$

H $0.0586422 .841055-0.748313$

H $1.717477-2.524146-1.239119$

H $3.569738-1.8503440 .231918$

H -2.713924-1.694459 0.574860

H -4.426034 $-0.508841-0.870858$

H -4.2025990 .9541590 .120391$

H $-4.842507-0.5020350 .855091$

$\mathrm{H}-2.888771-0.5646542 .423013$

SCF Energy $\left(B 3 L Y P / 6-31 G^{* *}\right)=-689.139244037$

03_c08

MMFF Geometry

O $0.9265910 .197897 \quad 1.469378$

C $-1.278533 \quad 1.0676720 .727521$

C $0.769571 \quad 1.624274-0.533197$

C $-0.5454491 .801560-0.353335$

C 1.6271450 .7275420 .328783

C $-0.462319-0.103674 \quad 1.311330$

C $-0.653663-1.3685340 .538388$

C $-1.676821-1.514596-0.310645$

C $-2.719037-0.485774-0.470906$

O $-2.5677320 .667305 \quad 0.221748$

H $-0.840895-0.3104392 .320558$

H -1.4709431 .7910061 .529547$

C $2.328843-0.383028-0.499036$
C $3.166022-1.308568 \quad 0.379349$

O $-3.704105-0.711435-1.161902$

H 2.4150151 .3695070 .744259

$\begin{array}{llll}\text { O } & 3.196313 & 0.208224 & -1.475995\end{array}$

H $1.2828172 .165576-1.325662$

H -1.102559 2.490331 -0.981569

H $\quad 0.046669-2.1818700 .695062$

H - $1.830994-2.429300-0.871206$

H $1.588112-0.979994-1.041428$

H $3.678769-2.057510-0.234094$

H $2.555013-1.8329831 .119199$

H $3.946257-0.7457200 .903449$

H $3.589656-0.515769-1.993457$

SCF Energy $\left(B 3 L Y P / 6-31 G^{* *}\right)=-689.138735504$

03_c09

MM̄MF Geometry

O $\quad 0.935237 \quad 0.125566 \quad 1.456514$

C -1.266712 1.0405780 .767305

C $0.7753111 .623375-0.493519$

C $-0.536357 \quad 1.806202-0.294071$

C 1.6297820 .6909900 .331766

C $-0.457174-0.161704 \quad 1.294923$

C $-0.664142-1.388188 \quad 0.466266$

C $-1.698066-1.488869-0.376589$

C $-2.736877-0.448751-0.474821$

$\begin{array}{llll}\text { O } & -2.565838 & 0.673758 & 0.262705\end{array}$

H $-0.830748-0.4099552 .296577$

H -1.440862 1.7349451 .598540

C $2.329152-0.390309-0.536743$

C $3.139996-1.3753690 .300995$

O $-3.736053-0.638877-1.156092$

H $2.421371 \quad 1.3151460 .766925$

O $3.2142740 .216881-1.488810$

H $1.2802712 .181782-1.279228$

H $-1.0951012 .516578-0.896487$

H $\quad 0.033730-2.2116490 .573209$

H $-1.862674-2.374896-0.978839$

H $1.587199-0.952909-1.112580$

H $2.508572-1.9403560 .992111$

H $3.910420-0.8537790 .879534$

H $3.662583-2.086141-0.348460$

H $3.9548180 .614888-0.998912$

SCF Energy $\left(B 3 L Y P / 6-31 G^{* *}\right)=-689.140100557$

03_c10

MMFF Geometry

O $0.9417340 .177604 \quad 1.482633$

C $-1.263881 \quad 1.0558200 .757856$

C $0.7917451 .646358-0.483270$

C $-0.5225101 .822360-0.295704$

C $1.641016 \quad 0.7072180 .341800$

C $-0.448477-0.122275 \quad 1.328742$

C $-0.645406-1.3819140 .550179$

C $-1.661082-1.517751-0.309185$

C $-2.691661-0.479790-0.480339$

$\begin{array}{lllll}\text { O } & -2.545091 & 0.665256 & 0.225872\end{array}$

H $-0.822603-0.3343442 .338534$

H -1.4699991 .7595851 .573918$

C $2.300614-0.408273-0.515293$

C $3.167060-1.3336510 .333465$

O $-3.662758-0.689177-1.195518$

H 2.4520651 .3200700 .756536

$\begin{array}{llll}\text { O } & 3.148156 & 0.149262 & -1.527507\end{array}$

H $1.3047662 .237706-1.238068$

H - $1.0753392 .546304-0.887735$

H $0.046054-2.2024440 .712183$

H $-1.816455-2.430579-0.872808$

H $1.543654-1.015387-1.022340$

H $2.582064-1.8556311 .095838$

H $3.967502-0.7726880 .828477$

H $3.655428-2.083281-0.298918$

H $2.5720320 .478519-2.238963$

SCF Energy $\left(B 3 L Y P / 6-31 G^{* *}\right)=-689.139770282$

03_c11

MMFF Geometry 
O $-0.912179-0.7621061 .470006$

C $1.144049-1.3360520 .198888$

C -1.041749-1.227981-0.952732

C $0.267540-1.504933-1.006074$

C $-1.747473-0.7568330 .296821$

C $0.482732-0.4972861 .309488$

C $0.744001 \quad 0.961727 \quad 1.147799$

C $1.7179421 .412564 \quad 0.350017$

C $2.6425950 .505894-0.348501$

O $2.422328-0.824367-0.227662$

H $0.948731-0.7757892 .263550$

H $1.332839-2.3423170 .593458$

C $-2.505696 \quad 0.5965390 .145271$

C $-3.7173470 .491975-0.776055$

O $3.5950050 .955874-0.972414$

H -2.511338-1.512502 0.521600

O $-1.6777201 .654259-0.349538$

H -1.650738 -1.387210 -1.839247

H $0.718487-1.885933-1.918054$

H $\quad 0.114775 \quad 1.6607751 .690586$

H 1.9067612 .4723340 .224657

H -2.844600 $0.915814 \quad 1.137575$

H $-4.408109-0.287166-0.440266$

H -3.420561 $0.281829-1.808808$

H $-4.2566611 .445488-0.798961$

$\mathrm{H}-1.2686231 .351719-1.178763$

SCF Energy $\left(B 3 L Y P / 6-31 G^{* *}\right)=-689.139757133$

$03 \_12$

MMFFF Geometry

O $-0.897925-0.7414211 .473476$

C $1.167188-1.3264310 .216768$

C $-1.007246-1.243954-0.948757$

C $0.303383-1.515962-0.992005$

$\begin{array}{llll}\text { C } & -1.725567 & -0.772170 & 0.292517\end{array}$

C $0.495131-0.4686141 .306254$

C $0.7453890 .989048 \quad 1.113886$

C $1.719737 \quad 1.4268550 .309147$

C $2.6585080 .509757-0.357059$

O $2.449999-0.819325-0.202035$

H $0.962207-0.7215532 .266975$

H $1.353020-2.3255440 .630435$

C -2.5189230 .5582840 .129205$

C -3.745407 $0.397130-0.764944$

O $3.6207880 .951936-0.972329$

H -2.470064 -1.544480 0.526038

O $-1.7088331 .602523-0.419478$

H -1.605113 -1.389554-1.844190

H $0.763961-1.885657-1.903099$

H 0.1049291 .6946121 .631738

H 1.8987082 .4835280 .151901

H -2.837976 0.8960281 .122464

H $-4.404563-0.396062-0.399741$

H -3.461308 $0.175829-1.798905$

H $-4.3171511 .331318-0.795916$

H -2.252041 2.408169-0.454859

SCF Energy $\left(B 3 L Y P / 6-31 G^{* \star}\right)=-689.135938322$

03_c13

MMFF Geometry

O $1.090969-0.250586-1.193781$

C -1.146908 -1.183530 -0.511890

C $0.783960-1.4446910 .982943$

C $-0.525621-1.6210510 .776291$

C $1.712968-0.863881-0.047914$

C $-0.328478-0.082774-1.213799$

C $-0.6778891 .276134-0.697324$

C $-1.8060751 .495736-0.012950$

C $-2.811753 \quad 0.440676 \quad 0.197617$

O $-2.512715-0.799270-0.257245$

H $-0.611304-0.074387-2.274403$

H $-1.189815-2.063203-1.165820$

C 2.7853620 .0912730 .539829

C 2.2446051 .3791741 .143978

O

H $2.269041-1.711418-0.470859$

O $3.7154320 .451740-0.490957$
H $1.227597-1.7998941 .910533$

H -1.148829-2.107691 1.520953

H $-0.0069542 .099894-0.915572$

H -2.071848 2.4800650 .354650

H $3.355219-0.4411581 .310505$

H $1.459593 \quad 1.1913211 .881381$

H 1.8680442 .0558360 .372864

H 3.0557091 .9219621 .643047

H $3.1745670 .648007-1.280801$

SCF Energy $\left(B 3 L Y P / 6-31 G^{* *}\right)=-689.137532188$

03_c14

MM̄FF Geometry

O $-0.891680-0.2804531 .514376$

C $1.142606-1.2521460 .409478$

C $-1.014762-1.253104-0.773654$

C $0.289555-1.550199-0.780298$

C $-1.734683-0.6510770 .405853$

C $0.521120-0.1876731 .332578$

C $\quad 0.887274 \quad 1.2017990 .922301$

C $1.897856 \quad 1.435646 \quad 0.077829$

C $2.7675520 .357137-0.423091$

O $2.465900-0.908010-0.047149$

H $0.956146-0.3176552 .332176$

H $1.244969-2.1877740 .973041$

C -2.680206 0.5335940 .050870

C $-3.908760 \quad 0.094193-0.738024$

O $3.7554450 .623535-1.094882$

H -2.374395 -1.4437810 .815119$

O $-2.0227091 .542141-0.721073$

H -1.610663 -1.496110-1.650063

H $0.745169-2.029987-1.641781$

H 0.3346552 .0294561 .355239

H $2.1791612 .439854-0.218047$

H -3.012264 1.0146440 .978736

H $-4.469320-0.679990-0.205675$

H -3.638719 -0.281326-1.730276

H $-4.5743800 .948586-0.904630$

$\mathrm{H}-1.241316 \quad 1.817347-0.217067$

SCF Energy $\left(B 3 L Y P / 6-31 G^{\star *}\right)=-689.137281645$

04_c01

MMFF Geometry

O $0.686364-0.607082-0.475457$

C $-1.1183691 .055498-0.670178$

C $1.2363121 .758808-0.317620$

C $-0.0681692 .076163-0.338395$

C $1.7510520 .360727-0.579928$

C $-0.466445-0.226262-1.231183$

C $-1.424730-1.364841-1.150985$

C $-2.474633-1.338923-0.321649$

C -2.700316 $-0.229790 \quad 0.622571$

O $\begin{array}{lllll} & -1.869389 & 0.835051 & 0.537985\end{array}$

H $-0.172911-0.073084-2.278062$

H - $-1.8077171 .479097-1.411690$

C $2.857366-0.0481330 .425348$

C $3.458520-1.4086060 .089175$

O $-3.591544-0.3044391 .458390$

H $2.1611870 .322621-1.597029$

O $2.325853-0.128223 \quad 1.753220$

H $1.9697652 .526852-0.083844$

H $-0.395107 \quad 3.084712-0.103434$

H - $1.243876-2.231541-1.778255$

H -3.168026 - $2.168492-0.247218$

H 3.6564590 .7017340 .434498

H $3.887425-1.415136-0.917319$

H $2.710718-2.2058340 .159916$

H $4.248456-1.6602420 .805347$

H $1.439835-0.5309891 .661063$

SCF Energy $\left(B 3 L Y P / 6-31 G^{\star \star}\right)=-689.147022914$

04_c02

MMFF Geometry

O $0.515865-0.745952-0.326089$

C - $-1.148326 \quad 1.031412-0.678948$

C $1.2437961 .585821-0.309413$

C $-0.0322861 .994589-0.394742$ 
C $1.6578950 .138444-0.440566$

C $-0.581706-0.327442-1.140269$

C $-1.628439-1.381572-1.017845$

C $-2.686322-1.225579-0.212982$

C $-2.838268-0.0486600 .661875$

$\begin{array}{lllll}\text { O } & -1.932727 & 0.948092 & 0.524665\end{array}$

H $-0.249213-0.264280-2.184682$

H -1.792331 $1.452077-1.461854$

C $2.659772-0.3299320 .643521$

C 4.0101180 .3684380 .578510

O $-3.740635-0.0075181 .488138$

H $2.098297-0.028115-1.431843$

O $2.894533-1.737630 \quad 0.478294$

H $2.0208002 .326416-0.141137$

H $-0.2880073 .041946-0.263907$

H -1.506472 -2.294185-1.591962

H $-3.444082-1.992856-0.105510$

H $2.223532-0.2027601 .641512$

H $3.932187 \quad 1.4353770 .803173$

H $4.4634490 .249413-0.411715$

H $4.705498-0.0808731 .296222$

H $2.002667-2.1312150 .396791$

SCF Energy $\left(B 3 L Y P / 6-31 G^{\star *}\right)=-689.148280809$

04_c03

MMFF Geometry

O $0.465505-0.992467-0.352071$

C $-1.0569170 .868077-0.891226$

C $1.3873311 .239367-0.730663$

C $0.1514021 .754774-0.819402$

C $1.663243-0.247179-0.640127$

C $-0.638581-0.592311-1.165681$

C $-1.765162-1.511018-0.834512$

C $-2.750206-1.138763-0.008561$

C $-2.739230 \quad 0.160937 \quad 0.687174$

O $-1.7659041 .038807 \quad 0.349881$

H $-0.367587-0.715568-2.222490$

H $-1.7133631 .224620-1.695390$

C $2.718639-0.6094990 .435668$

C $2.345838-0.1427361 .841108$

$\begin{array}{lllll}\text { O } & -3.574857 & 0.404923 & 1.548244\end{array}$

H $2.045089-0.573447-1.616467$

O $3.996249-0.070150 \quad 0.079452$

H $2.2507451 .900837-0.710778$

H $0.0002512 .829413-0.844812$

$\mathrm{H}-1.760098-2.504701-1.269876$

H $-3.563277-1.8058190 .252399$

H $2.822287-1.7014340 .458605$

H $3.090692-0.4929032 .564372$

H $1.366689-0.5232032 .146569$

H 2.3407400 .9500041 .911740

H $4.621737-0.326591 \quad 0.779427$

SCF Energy $\left(B 3 L Y P / 6-31 G^{* *}\right)=-689.144488582$

04_c04

MMFF Geometry

O $0.464426-0.879785-0.601565$

C $-1.0390201 .068289-0.649445$

C $1.4102471 .365976-0.408394$

C $0.1787791 .897941-0.364360$

C $1.670889-0.100646-0.689053$

C $-0.635571-0.278664-1.285356$

C $-1.770964-1.240229-1.190614$

C $-2.749275-1.079495-0.291488$

C -2.7220280 .0022190 .709897$

$\begin{array}{llll}\text { O } & -1.739685 & 0.927116 & 0.599539\end{array}$

H $-0.366568-0.134388-2.339930$

$\mathrm{H}-1.695177 \quad 1.621581-1.333604$

C $2.713819-0.7347710 .268124$

C $2.303594-0.7029001 .739481$

O -3.5519520 .0301251 .609585$

H $2.057929-0.176515-1.713647$

O $3.997904-0.1210140 .108959$

H $2.2732642 .005038-0.242791$

H $0.0357532 .948462-0.130441$

H - $1.777599-2.092261-1.862296$

H -3.568257-1.783471 -0.202437
H $2.833657-1.787487-0.015489$

H $1.348106-1.2101231 .902498$

H 2.2243250 .3237052 .112296

H $3.065935-1.1961292 .352547$

H 4.0402810 .6437290 .708400

SCF Energy $\left(B 3 L Y P / 6-31 G^{* *}\right)=-689.145445514$

04_c05

MMFF Geometry

O $-0.476298 \quad 1.042629-0.087455$

C $1.048745-0.626897-1.071431$

C $-1.392681-1.043340-0.973521$

C $-0.155764-1.512722-1.198968$

C $-1.6719590 .375515-0.526976$

C $0.6220960 .853987-0.981810$

C $1.7481681 .673865-0.450589$

C $2.746514 \quad 1.1202220 .247922$

C $2.753398-0.3098950 .606023$

$\begin{array}{lllll}\text { O } & 1.776438 & -1.086293 & 0.082065\end{array}$

H $0.3398491 .225416-1.975731$

H $1.694444-0.775525-1.946486$

C -2.7191940 .4666270 .613716$

C $-2.350143-0.3470341 .850542$

O $3.606623-0.7498121 .365940$

H -2.067242 $0.921142-1.393933$

$\begin{array}{llll}\text { O } & -4.010050 & 0.040725 & 0.163396\end{array}$

H -2.250282 -1.702560 -1.091184

H $0.000123-2.550766-1.476311$

H $1.7325312 .743671-0.630170$

H 3.5597221 .7106090 .653410

H -2.810577 1.5147220 .925382

H - $1.367889-0.0646542 .240822$

H -2.353727 -1.4218291 .640798$

H $-3.093547-0.1875082 .639589$

H -4.332187 $0.707323-0.467208$

SCF Energy $\left(B 3 L Y P / 6-31 G^{* *}\right)=-689.145593548$

04_c06

MMFF Geometry

O $0.9398820 .124151-1.463718$

C $-1.2529521 .052943-0.752958$

C 0.8067051 .6320160 .488179

C -0.5060721 .8169190 .298385$

C $1.6470720 .691805-0.334630$

C $-0.455915-0.148542-1.299420$

C $-0.669606-1.386458-0.490975$

C $-1.694010-1.4901260 .362844$

C $-2.716865-0.4387520 .496271$

$\begin{array}{llll}O & -2.545820 & 0.691347 & -0.229043\end{array}$

H $-0.835470-0.378661-2.303201$

H $-1.4380941 .750139-1.579546$

C $2.321940-0.447700 \quad 0.473232$

C 3.3147340 .0517151 .514499

O $-3.702510-0.6237701 .198064$

H $2.4477291 .284168-0.795489$

O $3.034892-1.308126-0.428013$

H $1.3161642 .217126 \quad 1.250290$

H -1.055187 2.5421540 .892554

H $0.017986-2.216596-0.619758$

H - $-1.861440-2.3855690 .950472$

H $1.571750-1.0683660 .971788$

H $2.825367 \quad 0.6391382 .296223$

H 4.0942350 .6644961 .048619

H $3.824593-0.7935431 .989940$

H 2.510643-1.326239-1.252769

SCF Energy $\left(B 3 L Y P / 6-31 G^{* *}\right)=-689.143655208$

04 c07

MMFF Geometry

O $0.481629-0.781170-0.278823$

C - $-1.1793560 .966562-0.736025$

C $1.2164341 .535298-0.414987$

C $-0.0593081 .942167-0.517051$

C $1.6307250 .078996-0.462493$

C $-0.617949-0.419663-1.112030$

C $-1.665381-1.461758-0.914362$

C $-2.718531-1.252998-0.115568$ 
C $-2.864880-0.0209890 .680131$

O -1.9619440 .9656720 .471926$

H $-0.292597-0.425303-2.160581$

H -1.823797 $1.338626-1.542828$

C $2.667560-0.3404120 .615274$

C 3.8846190 .5704820 .716243

O -3.7630260 .0753891 .506872$

H $2.047426-0.138637-1.454565$

$\begin{array}{llll}\text { O } & 3.162721 & -1.651926 & 0.290844\end{array}$

H $1.9941682 .286423-0.312986$

H $-0.3111432 .996554-0.455100$

H $-1.541558-2.411903-1.423377$

H $-3.473217-2.0129470 .048572$

H $2.172142-0.4166691 .591067$

H 3.6252081 .5584391 .106134

H $4.3653840 .689322-0.260952$

H 4.6356330 .1329101 .383608

H $3.483721-2.0466401 .119670$

SCF Energy $\left(B 3 L Y P / 6-31 G^{\star *}\right)=-689.139895463$

04_c08

MMFF Geometry

O $0.657474-0.650122-0.501355$

C $-1.143677 \quad 1.027126-0.694817$

C $1.2193251 .726831-0.416435$

C $-0.0826472 .053599-0.424657$

C $1.7192540 .313770-0.628715$

C $-0.514151-0.278128-1.226571$

C $-1.478824-1.405750-1.085260$

C $-2.506388-1.344266-0.230446$

C $-2.705670-0.2000590 .677046$

O -1.8815160 .8624890 .529806$

H $-0.251100-0.160258-2.286142$

H $-1.842014 \quad 1.428938-1.440348$

C $2.871535-0.055437 \quad 0.346725$

C $3.355561-1.4874050 .134276$

O $-3.582613-0.239604 \quad 1.531104$

H $2.1093080 .241812-1.652565$

$\begin{array}{lllll}\text { O } & 2.460340 & 0.093117 & 1.710124\end{array}$

H $1.9620952 .495550-0.220956$

H $-0.3986753 .071550-0.219516$

H - $1.315680-2.296287-1.682686$

H -3.199846 -2.167649-0.107440

H 3.7101050 .6332110 .188638

H $3.656559-1.655198-0.904164$

H $2.581061-2.2140240 .402160$

H $4.214770-1.698127 \quad 0.780513$

H $3.138022-0.3082472 .278182$

SCF Energy (B3LYP/6-31G**) $=-689.139700760$

04_c09

MMFF Geometry

$\begin{array}{llll}O & -1.130020 & 0.592534 & 1.253231\end{array}$

C $1.1494911 .241844 \quad 0.505020$

C $-0.7888831 .562677-0.994402$

C $0.5205511 .727329-0.767053$

C $-1.7341460 .917728-0.009343$

$\begin{array}{llll}\text { C } & 0.256312 & 0.247742 & 1.272721\end{array}$

C $0.471707-1.1603430 .830642$

C $1.555115-1.5145920 .131282$

C $2.637210-0.564807-0.172994$

$\begin{array}{lllll}\text { O } & 2.460361 & 0.723538 & 0.203490\end{array}$

H $0.553756 \quad 0.2739722 .328995$

H 1.3059492 .1251901 .136680

C $-2.536581-0.264924-0.628636$

$\begin{array}{llll}\text { C }-3.484551 & -0.900701 & 0.381242\end{array}$

O $3.667922-0.953638-0.706944$

H -2.4786721 .6847420 .241930$

O $-1.705846-1.307821-1.146213$

H -1.221138 1.942182 -1.917581

H $1.1507282 .239636-1.488572$

H $-0.279652-1.9013521 .086775$

H $1.714147-2.535826-0.194895$

H -3.125638 $0.111294-1.474024$

H $-4.169208-0.1602700 .805596$

H $-2.934180-1.3820341 .196647$

H -4.077694-1.687160 -0.098354
$\mathrm{H}-1.055086-0.898987-1.742583$

SCF Energy $\left(B 3 L Y P / 6-31 G^{* *}\right)=-689.141204626$

04_c10

MMFF Geometry

O $\begin{array}{llll}-1.108379 & 0.632160 & 1.244184\end{array}$

C 1.1751801 .2463720 .471853

C $-0.7583591 .545844-1.031165$

C $0.552496 \quad 1.707555-0.810318$

C $-1.7087220 .941273-0.026461$

$\begin{array}{llll}\text { C } & 0.275285 & 0.273749 & 1.259202\end{array}$

C $0.476200-1.1439770 .841293$

C $1.553987-1.5171340 .142885$

C $2.645816-0.582268-0.173439$

$\begin{array}{llll}\text { O } & 2.484056 & 0.712783 & 0.188373\end{array}$

H 0.5789990 .3145852 .313308

H 1.3351952 .1421731 .084839

C $-2.547650-0.234397-0.607462$

C $-3.481579-0.8395350 .435534$

O $3.678140-0.991351-0.690147$

H -2.4338831 .7307460 .212358$

O $-1.725453-1.277750-1.136671$

H -1.184659 $1.882031-1.972727$

H $1.1876922 .179610-1.553597$

H $-0.284288-1.8715251 .103136$

H $1.700136-2.541645-0.176996$

H -3.144728 $0.147071-1.445017$

H - $-4.133027-0.0784880 .875407$

H -2.919099-1.331173 1.236263

H $-4.111048-1.611926-0.019848$

H -2.314116 -1.953083 -1.514560

SCF Energy (B3LYP/6-31G**) $=-689.136801182$

04_c11

MMFF Geometry

O $-1.094745 \quad 0.279744 \quad 1.227293$

C $1.161841 \quad 1.171900 \quad 0.571929$

C $-0.770677 \quad 1.532032-0.902580$

C $0.5387201 .692086-0.682417$

C $-1.713108 \quad 0.8819190 .077046$

C 0.3179910 .0690951 .239398

C $0.632563-1.2874290 .696523$

C $1.742181-1.513448-0.015473$

C $2.764759-0.474158-0.225959$

$\begin{array}{lllll}O & 2.510511 & 0.756674 & 0.277984\end{array}$

H 0.6003270 .0283232 .299563

H 1.2440282 .0183991 .264849

C $-2.704639-0.097858-0.611715$

C $-3.700666-0.7014320 .373649$

O $3.823126-0.751759-0.774770$

H -2.3279321 .6936350 .488172$

O -2.029650 -1.169294 -1.274980

H $-1.2056821 .925761-1.818628$

H $1.1644192 .213067-1.401167$

H $-0.043388-2.104500 \quad 0.923561$

H $1.984218-2.495166-0.405957$

H -3.261563 $0.443788-1.385462$

H -4.2641680 .0774570 .895958$

H -3.198235 - 1.3316911 .115315

H - $-4.409352-1.349036-0.154123$

H - $1.570387-1.679200-0.588715$

SCF Energy $\left(B 3 L Y P / 6-31 G^{* *}\right)=-689.137239945$

04_c12

MM̄FF Geometry

$\begin{array}{llll}0 & 0.895199 & 0.327001 & -1.504196\end{array}$

C $-1.2855801 .128107-0.644142$

C 0.8098721 .6148830 .581341

C $-0.508597 \quad 1.8074180 .443559$

C $1.6298380 .731806-0.328392$

C $-0.4891510 .013270-1.350585$

C $-0.673346-1.321062-0.707700$

C $-1.661095-1.5453210 .164889$

C $-2.675049-0.5266710 .483274$

O $-2.546093 \quad 0.686772-0.101175$

H $-0.887066-0.090640-2.368266$

H $-1.5251001 .899981-1.386321$ 
C $2.284118-0.4835040 .393621$

C $3.181096-0.0772971 .560704$

O $-3.620482-0.8069631 .208959$

H $2.4472641 .345693-0.727775$

O $3.092589-1.217032-0.537297$

H 1.3397392 .1475021 .367148

H $-1.0410612 .485828 \quad 1.104107$

H $0.014063-2.116083-0.980436$

$\mathrm{H}-1.803505-2.5128060 .631995$

H $1.518085-1.1719690 .760626$

H 2.6135880 .3922402 .368836

H 3.9630470 .6143951 .228665

H $3.693472-0.953664 \quad 1.973086$

H $3.425132-2.008470-0.081415$

SCF Energy (B3LYP/6-31G**)= -689.135818917

04_c13

MMFF Geometry

O $0.882153-0.2700541 .499640$

C $-1.154409-1.241997 \quad 0.385074$

C $0.990277-1.215295-0.807410$

C $-0.316700-1.498198-0.825732$

C $1.714755-0.6798140 .402549$

C $-0.531767-0.186314 \quad 1.318517$

C -0.9019821 .2026910 .909034$

C $-1.927787 \quad 1.4384750 .083750$

C $-2.8080190 .360684-0.398311$

O $-2.490531-0.905976-0.040587$

H $-0.966823-0.3211452 .317351$

H $-1.233587-2.1909460 .929683$

C 2.7690220 .4161510 .079545

C $2.2272271 .635777-0.656447$

O $-3.8129810 .627714-1.044268$

H $2.286835-1.534050 \quad 0.790308$

O $3.825275-0.150671-0.709080$

H $1.600357-1.437324-1.680981$

H $-0.778433-1.944209-1.701435$

H $-0.3318852 .028717 \quad 1.320455$

H -2.208488 $2.442914-0.210597$

H 3.2159830 .7542101 .022503

H $1.5039322 .184558-0.052706$

H $1.7629791 .359523-1.608573$

H $3.0442702 .323632-0.902445$

H $4.5098310 .533052-0.812617$

SCF Energy $\left(B 3 L Y P / 6-31 G^{* *}\right)=-689.134493914$

\section{5_c01}

MMFF Geometry

O $-0.728105-1.038017-0.20932$

$\begin{array}{lllll}\text { C } & 0.929925 & 0.627929 & 0.331424\end{array}$

C $-1.3226641 .352024-0.339472$

C -0.0998241 .6930490 .105644$

C $-1.736663-0.082663-0.644046$

C $0.578996-0.581190-0.546705$

C $1.603103-1.629963-0.266416$

C $2.849232-1.254276 \quad 0.062519$

C 3.2453540 .1773700 .098933

$\begin{array}{lllll}\text { O } & 2.244042 & 1.099640 & 0.021638\end{array}$

H $0.625219-0.353476-1.621511$

H $\quad 0.896300 \quad 0.381367 \quad 1.403020$

C -3.062455 $-0.526241 \quad 0.025080$

C $-4.2700590 .312868-0.362823$

$\begin{array}{lllll}\text { O } & 4.426068 & 0.491976 & 0.160665\end{array}$

H -1.841918 $-0.185552-1.731631$

O $-2.932746-0.5083061 .452067$

H $-2.0590022 .133971-0.505569$

H 0.1512292 .7240540 .333117

H $1.333551-2.676378-0.355079$

H $3.640957-1.9705640 .247589$

H $-3.255859-1.570766-0.248240$

H $-4.3889320 .362246-1.449191$

H $-4.202209 \begin{array}{lll}1.329626 & 0.037121\end{array}$

H $-5.180747-0.1209280 .065157$

H -2.082338 -0.9493851 .640003$

SCF Energy $\left(B 3 L Y P / 6-31 G^{* *}\right)=-689.144820855$

05_c02
MMFF Geometry

O $-0.892939-0.796382-0.185352$

C $0.940608 \quad 0.712693 \quad 0.250420$

C $-1.1887711 .633594-0.563008$

C $0.0577691 .868697-0.116442$

C $-1.7667540 .236556-0.718724$

C $0.466299-0.528435-0.520173$

C $1.350900-1.657809-0.108715$

C $2.624481-1.4017830 .229468$

C $3.189659-0.0302740 .149952$

O $2.3102540 .991795-0.052091$

H $0.562032-0.413419-1.609530$

H 0.8485340 .5804591 .338650

C $-3.149418 \quad 0.069826-0.043475$

C $-3.1161580 .228064 \quad 1.473806$

$\begin{array}{lrlll}O & 4.397355 & 0.148063 & 0.230726\end{array}$

H $-1.8786900 .024004-1.789837$

O $-3.655321-1.240636-0.333378$

H $-1.8265122 .469527-0.840918$

H 0.4360332 .8787260 .004071

H $0.960501-2.669277-0.111532$

H $3.318933-2.1857510 .507528$

H -3.859129 $0.790144-0.465575$

H -2.781149 1.2261001 .770219

H -2.460724 -0.5160251 .939100$

H -4.1156970 .0633651 .891302$

H -2.930610 -1.857356-0.111374

SCF Energy $\left(B 3 L Y P / 6-31 G^{* *}\right)=-689.145096842$

05 c03

MMFF Geometry

O $-\begin{array}{lll}0.755387 & 0.872775 & -0.011772\end{array}$

C $1.050579-0.683310-0.373638$

C $-1.173288-1.545408 \quad 0.213466$

C $0.094042-1.815127-0.147202$

C $-1.709314-0.1306520 .413850$

C $\quad 0.5595050 .535670 \quad 0.420504$

C $1.5168021 .646700 \quad 0.142928$

C $2.804121 \quad 1.354957-0.101007$

C $3.308672-0.041343-0.046471$

O $2.378093-1.0350380 .026329$

H $\quad 0.5600530 .3635191 .506620$

H $1.057969-0.491555-1.456904$

C $-3.0392540 .087038-0.350584$

C $-3.5573201 .515469-0.204710$

O $4.511669-0.264831-0.035316$

H -1.8895780 .0046451 .488616$

O $-4.045030-0.8052050 .144082$

H - $1.871394-2.3612280 .389784$

H $0.434432-2.834017-0.298826$

H 1.1634552 .6713820 .165102

H $3.5482412 .121968-0.279633$

H $-2.891322-0.125844-1.416489$

H -2.860146 $2.245898-0.625373$

H -3.7307201 .7602460 .848955$

H $-4.5199131 .627731-0.715566$

$\mathrm{H}-4.843111-0.668318-0.395671$

SCF Energy $\left(B 3 L Y P / 6-31 G^{* *}\right)=-689.141923235$

05_c04

MMFF Geometry

O $-0.763347 \quad 0.867950-0.011211$

C $1.050800-0.678831-0.387024$

C $-1.175619-1.5554740 .171587$

C $0.095489-1.815937-0.182531$

C $-1.715361-0.1455490 .386649$

C $0.551267 \quad 0.5281510 .419938$

C 1.5066431 .6454390 .161992

C $2.7965841 .360008-0.075864$

C $3.304541-0.035548-0.032284$

O $2.375722-1.032340 \quad 0.020019$

H 0.5476360 .3413201 .503645

H $1.066400-0.472746-1.467534$

C $-3.0447120 .075467-0.379936$

C -3.549778 $1.512095-0.273658$

O $4.507864-0.256064-0.010036$

H -1.904334 -0.0275771 .462000$ 
O $-4.068045-0.7986890 .114170$

H - $-1.873347-2.3775600 .318437$

H $0.438526-2.831189-0.352588$

H 1.1501832 .6688800 .191175

H $3.5398422 .130822-0.241416$

H -2.900049 -0.157496-1.441675

H $-2.8557312 .220973-0.734544$

H -3.6978941 .7976270 .773596$

H $-4.5224321 .611046-0.767852$

H -4.325459-0.489885 1.000022

SCF Energy $\left(B 3 L Y P / 6-31 G^{\star \star}\right)=-689.143476375$

\section{5 c05}

MMFF Geometry

O

C $1.037359-0.682552-0.361797$

C $-1.188450-1.5275180 .249159$

C $0.076764-1.806795-0.112047$

C $-1.719789-0.1061190 .420593$

C $0.556908 \quad 0.546924 \quad 0.422057$

C 1.5190981 .6495170 .129211

C $2.8035641 .348646-0.118638$

C $3.300809-0.049662-0.053589$

O $2.364947-1.0373250 .034374$

H 0.5593480 .3859691 .509825

H $1.038133-0.504662-1.447376$

C $-3.0372970 .120102-0.364435$

C $-3.5763461 .534080-0.166670$

O $4.502339-0.280562-0.047286$

H -1.910292 0.0455801 .491288

$\begin{array}{lllll} & \mathrm{O} & -4.060651 & -0.784435 & 0.063931\end{array}$

H - $1.872849-2.3437700 .465758$

H $0.419775-2.829037-0.234128$

H 1.1715612 .6764640 .143434

H $3.5507192 .110095-0.308349$

H $-2.869881-0.031341-1.438270$

H -2.881076 $2.290842-0.541521$

H $-3.773506 \quad 1.7317190 .892789$

H -4.530886 $1.654120-0.690917$

H $-3.936427-1.613329-0.429948$

SCF Energy $\left(B 3 L Y P / 6-31 G^{\star *}\right)=-689.142910349$

\section{5 c06}

MMFF Geometry

O $-0.865406-0.784039-0.159214$

C 0.9785830 .7010990 .274225

C $-1.1595051 .644560-0.488954$

C $0.093393 \quad 1.867467-0.053268$

C $-1.7452030 .248920-0.672734$

C $0.487142-0.521448-0.513625$

C $1.372834-1.662139-0.137250$

C $2.652723-1.4187970 .185574$

C $3.222201-0.0482980 .123355$

O $2.3446250 .981911-0.043544$

H $0.566959-0.386017-1.601922$

H 0.9034880 .5468021 .360919

C -3.139725 $0.107984-0.007734$

C -3.074678 -0.0086891 .512908$

$\begin{array}{lllll}\text { O } & 4.431954 & 0.123799 & 0.188623\end{array}$

H -1.855297 $0.064779-1.749463$

O $-3.805996-1.046934-0.535715$

H - $1.7957462 .488380-0.744496$

H 0.4765792 .8733480 .083260

H $0.973378-2.670093-0.152294$

H $3.346821-2.2113590 .438291$

H $-3.7606270 .970403-0.277827$

H -2.5592680 .8450351 .962219$

H $-2.570036-0.9307851 .820472$

H $-4.085798-0.0570511 .932183$

$\mathrm{H}-4.608454-1.195293-0.007526$

SCF Energy $\left(B 3 L Y P / 6-31 G^{* *}\right)=-689.139768431$

05_c07

MMFF Geometry

O - $-0.699832-1.072375-0.205117$

$\begin{array}{llll}\text { C } & 0.939122 & 0.628533 & 0.302623\end{array}$

C $-1.3030441 .322631-0.415758$
C $-0.081429 \quad 1.6863200 .012927$

C $-1.720569-0.129126-0.627190$

C $0.603294-0.610251-0.538962$

C $1.631527-1.644685-0.219604$

C $2.871299-1.2556820 .116175$

C $3.260993 \quad 0.177103 \quad 0.118217$

O $2.2588251 .092656-0.002489$

H $0.657584-0.414649-1.619702$

H $0.8867950 .422278 \quad 1.381735$

C -3.036827 -0.5204340 .097687$

C $-4.2642650 .188676-0.460613$

$\begin{array}{lllll}O & 4.439971 & 0.497796 & 0.187837\end{array}$

H $-1.859944-0.285355-1.704745$

O $-2.948610-0.239648 \quad 1.500586$

H $-2.0416782 .092223-0.620363$

H 0.1718892 .7266500 .186268

H $1.364792-2.693757-0.278652$

H $3.661802-1.9637730 .333718$

H $-3.172121-1.6049610 .003651$

H $-4.3609830 .028724-1.538546$

H $-4.234741 \quad 1.264540-0.260710$

H $-5.170687-0.1878320 .026415$

H -3.706142 -0.670041 1.931383

SCF Energy $\left(B 3 L Y P / 6-31 G^{* *}\right)=-689.138342049$

06_c01

MMFF Geometry

O $0.766466-0.756948-0.860803$

C $-1.0617090 .782503-0.479449$

C $1.2447861 .629061-0.396752$

C $-0.0711781 .897554-0.331302$

C $1.8072800 .235182-0.618999$

C $-0.386125-0.527251-0.052850$

C $-1.392546-1.613720-0.241208$

C -2.695631-1.332680 -0.082525

C -3.1545640 .0184600 .330723$

$\begin{array}{lllll}O & -2.228547 & 1.019120 & 0.313173\end{array}$

H $-0.104676-0.5267041 .008990$

H - $-1.3617850 .765941-1.537626$

C $2.708821-0.2851230 .531307$

C 3.9485640 .5652830 .767978

$\begin{array}{lllll}\text { O } & -4.307278 & 0.195865 & 0.700133\end{array}$

H $2.3988660 .246824-1.542495$

O $3.148505-1.6147550 .216551$

H $1.9566962 .447616-0.322082$

H $-0.4365962 .912792-0.213814$

H - $1.057889-2.616599-0.481606$

H $-3.467339-2.087275-0.178805$

H $2.137700-0.3608211 .462920$

H 3.6939081 .5708511 .113785

H $4.5457720 .647671-0.146755$

H $4.5928790 .097701 \quad 1.520750$

H $2.383116-2.059871-0.197235$

SCF Energy $\left(B 3 L Y P / 6-31 G^{* *}\right)=-689.145248327$

06_c02

MMFF Geometry

O $0.914625-0.574262-1.003845$

C $-0.996548 \quad 0.809236-0.486966$

C $1.2502501 .801057-0.398630$

C $-0.077767 \quad 1.977774-0.284271$

C $1.8901330 .455967-0.719312$

C $-0.232812-0.482552-0.165873$

C $-1.175887-1.611195-0.426613$

C $-2.490536-1.424951-0.228981$

C -3.022533 -0.1395130 .292278$

$\begin{array}{llll}\text { O } & -2.160851 & 0.916820 & 0.336544\end{array}$

H $0.055269-0.5467950 .889925$

H - $-1.3171830 .845069-1.538891$

$\begin{array}{lll}\text { C } 2.908126 & 0.012656 & 0.371117\end{array}$

C $3.534134-1.3381270 .045613$

O $-4.177805-0.0608690 .687855$

H $2.4502180 .581551-1.654354$

O $2.313602-0.0989101 .668944$

H $1.9172642 .652084-0.283803$

H $-0.5067062 .956002-0.092813$

H $-0.780454-2.569859-0.742903$ 
H $-3.215410-2.217615-0.370302$

H 3.7049130 .7623010 .448623

H $4.017978-1.326687-0.935609$

H $2.786474-2.1384910 .063066$

H $4.284706-1.5994410 .799743$

H 1.9670020 .7775291 .911162

SCF Energy $\left(B 3 L Y P / 6-31 G^{\star *}\right)=-689.144171155$

\section{$06 \mathrm{c03}$}

MMFF Geometry

O $0.903908-0.533967-1.009034$

C -1.017125 $0.826640-0.460247$

C $1.2183201 .831491-0.339871$

C $-0.1111481 .997185-0.225398$

C $1.8684750 .503599-0.706615$

C $-0.240675-0.463476-0.162367$

C $-1.172002-1.598348-0.441599$

C $-2.489160-1.429683-0.246844$

C $-3.038283-0.158666 \quad 0.290371$

$\begin{array}{lllll}\text { O } & -2.190859 & 0.906809 & 0.354307\end{array}$

H $0.051840-0.5431660 .891216$

$\mathrm{H}-1.3300250 .881596-1.513640$

C 2.9115090 .0330870 .347323

C $3.560471-1.288554-0.051219$

O $-4.197443-0.1010390 .679086$

H $2.4113700 .665674-1.646383$

O $2.318006-0.1308951 .639508$

H $1.8801682 .677516-0.175664$

H -0.5450302 .9626200 .011188$

H $-0.765249-2.547495-0.771116$

H $-3.204469-2.228029-0.403159$

H 3.6870590 .8020840 .446575

H $4.019632-1.223475-1.042165$

H $2.831134-2.105735-0.050387$

H $4.336133-1.5647670 .671450$

H $3.016250-0.4237472 .249944$

SCF Energy $\left(B 3 L Y P / 6-31 G^{\star *}\right)=-689.140880249$

\section{6 c04}

MM̄FF Geometry

O $0.916062-0.561673-0.869322$

C $-1.0369140 .824006-0.443082$

C $1.1865881 .828617-0.234187$

C $-0.1452081 .990956-0.154837$

C $1.8518700 .519918-0.636018$

C $-0.269571-0.454820-0.083918$

C $-1.180589-1.604407-0.367263$

C $-2.506749-1.440136-0.243891$

C $-3.090906-0.158913 \quad 0.225938$

$\begin{array}{lllll}0 & -2.250923 & 0.910237 & 0.309844\end{array}$

H $-0.019500-0.4975420 .983637$

H -1.290937 $0.869213-1.512142$

C 2.9727170 .0796740 .345020

C $3.775769-1.097218-0.203657$

O $-4.269200-0.0976440 .549966$

H $2.3284380 .704273-1.607391$

O $2.446618-0.2970591 .620884$

H $1.8405742 .673948-0.033989$

H -0.5872052 .9495530 .095481$

H $-0.756680-2.560991-0.651599$

H -3.208860 -2.247928 -0.412793

H 3.6557190 .9195830 .517150

H $4.238234-0.848490-1.163562$

H $3.146431-1.983837-0.336576$

H $4.566020-1.3781170 .501076$

H $2.005278-1.1566191 .505097$

SCF Energy $\left(B 3 L Y P / 6-31 G^{* *}\right)=-689.141526082$

06_c05

MMFF Geometry

O $0.719515-0.840315-0.978234$

C $-1.0005200 .795534-0.430262$

C $1.3483691 .470188-0.298114$

C $0.0584801 .813443-0.143547$

C $1.8030950 .097969-0.777243$

C $-0.410197-0.591822-0.146784$

C $-1.487052-1.588826-0.426261$
C $-2.770122-1.243879-0.237738$

C $-3.1445540 .087055 \quad 0.302092$

O $-2.157153 \quad 1.0223200 .382192$

H $-0.130817-0.7073640 .907461$

H $-1.2895490 .917405-1.484270$

C $2.935548-0.5391350 .075120$

C $2.584040-0.785877 \quad 1.537555$

$\begin{array}{llll}O & -4.286245 & 0.300800 & 0.686762\end{array}$

H $2.2282820 .262657-1.776314$

$\begin{array}{lllll}\text { O } & 4.104570 & 0.288461 & 0.019649\end{array}$

H $2.1306502 .205204-0.119680$

H -0.2305892 .8165750 .151340$

H $-1.214773-2.583567-0.761119$

H $-3.586555-1.937050-0.401631$

H $3.200391-1.503162-0.376435$

H $1.785791-1.5249341 .640637$

H 2.2837420 .1392562 .039874

H $3.458306-1.1620852 .080776$

H $4.817098-0.1809560 .487490$

SCF Energy $\left(B 3 L Y P / 6-31 G^{* *}\right)=-689.139015740$

06_c06

MMFF Geometry

O $0.726413-0.851449-0.956577$

C $-0.993248 \quad 0.789727-0.433066$

C $1.3575711 .459725-0.284340$

C $0.0667991 .807363-0.146384$

C $1.8101310 .081499-0.744760$

C $-0.409193-0.597290-0.133938$

C $-1.486127-1.593339-0.415507$

C $-2.769890-1.243196-0.241490$

C $-3.146108 \quad 0.093544 \quad 0.282897$

O $-2.156519 \quad 1.026367 \quad 0.366194$

H $-0.137679-0.7065830 .922922$

H $-1.272250 \quad 0.903857-1.490684$

C $2.930229-0.5553110 .124623$

C $2.582083-0.7381341 .596313$

$\begin{array}{llll}\text { O } & -4.291664 & 0.314791 & 0.651323\end{array}$

H $2.2477870 .236494-1.739949$

$\begin{array}{lllll}\text { O } & 4.125816 & 0.233105 & 0.059598\end{array}$

H $2.1380452 .193538-0.093503$

H -0.2230972 .8122080 .142385$

H - $1.213876-2.592371-0.737419$

H -3.586681-1.935863 -0.406038

H $3.172071-1.545237-0.281638$

H $1.768950-1.4550791 .732783$

H 2.3057510 .2110232 .066600

H $3.453398-1.1117712 .146335$

H $4.4617150 .179743-0.852173$

SCF Energy $\left(B 3 L Y P / 6-31 G^{* *}\right)=-689.140419621$

06_c07

MM̄FF Geometry

O $0.722137-0.838132-1.004446$

C $-0.9854980 .798467-0.435214$

C $1.3711241 .461819-0.317734$

C $0.0829511 .810372-0.158258$

C $1.8170500 .082685-0.789469$

C $-0.401948-0.593473-0.164924$

C $-1.486902-1.580963-0.445333$

C $-2.765931-1.229068-0.241680$

$\begin{array}{llll}C & -3.125815 & 0.099725 & 0.313977\end{array}$

O

H - $0.118968-0.7176330 .887184$

H - $-1.2857230 .926780-1.485394$

C $2.930505-0.5698520 .078362$

C $2.546163-0.873761 \quad 1.521871$

$\begin{array}{llll}\text { O } & -4.261450 & 0.318386 & 0.713204\end{array}$

H $2.2582780 .242286-1.782312$

$\begin{array}{lllll}\mathrm{O} & 4.112041 & 0.242078 & 0.076208\end{array}$

H $2.1476032 .207282-0.165811$

H -0.2025252 .8190040 .122113$

H - $1.223918-2.574336-0.791794$

H -3.588639-1.915135 -0.404391

H $3.207675-1.520812-0.392371$

H $1.771765-1.6429711 .578015$

H 2.1961490 .0238442 .041795 
H $3.418881-1.2347172 .077719$

H 3.9956220 .9469280 .736252

SCF Energy $\left(B 3 L Y P / 6-31 G^{\star *}\right)=-689.139893099$

06_c08

MMFF Geometry

O $0.728713-0.770039-0.909762$

C $-1.082270 \quad 0.766089-0.493152$

C $1.2312161 .599549-0.453245$

C $-0.0824411 .876755-0.371526$

C $1.787840 \quad 0.195394-0.657772$

C $-0.406012-0.547107-0.081715$

C $-1.421862-1.626647-0.258812$

C $-2.719896-1.339737-0.072503$

C -3.1605870 .0115520 .360026$

O $-2.230531 \quad 1.0086090 .324930$

H $-0.110915-0.5517560 .976187$

H $-1.4064340 .750675-1.544352$

C $2.697367-0.3073430 .502588$

C $3.8245730 .656457 \quad 0.858244$

$\begin{array}{llll}\text { O } & -4.302653 & 0.192868 & 0.760132\end{array}$

H $2.3841230 .200246-1.578507$

O $3.302991-1.5511120 .119742$

H $1.9471652 .416465-0.409496$

H $-0.4405782 .895952-0.268980$

H $-1.092338-2.627281-0.515723$

H $-3.497408-2.089072-0.160210$

H $2.095244-0.5131021 .393972$

H 3.4448231 .5929901 .275877

H $4.4357970 .883016-0.022237$

H 4.4958510 .2051451 .597369

H $3.748946-1.9156600 .902955$

SCF Energy $\left(B 3 L Y P / 6-31 G^{\star *}\right)=-689.137246136$

07_c01

MM̄FF Geometry

O $0.760244-0.7118800 .906849$

C $-1.0808380 .792936 \quad 0.463814$

C $1.215366 \quad 1.654831 \quad 0.366654$

C $-0.101734 \quad 1.912966 \quad 0.286048$

C 1.7911510 .2684740 .634290

C $-0.386840-0.5222840 .083752$

C $-1.382075-1.6140490 .299629$

C $-2.687065-1.3540970 .123109$

C $-3.158103-0.022865-0.337864$

O $-2.2451540 .989538-0.344390$

H - $-0.096037-0.553299-0.975415$

H -1.3885300 .8068191 .519816$

C $2.718309-0.201697-0.519248$

C $3.242565-1.616197-0.289453$

$\begin{array}{lllll}0 & -4.309291 & 0.126937 & -0.724453\end{array}$

H $2.3909790 .343394 \quad 1.549956$

O $3.8399120 .681075-0.637982$

H 1.9348962 .4631640 .253142

H -0.4719402 .9203920 .127710$

H -1.035378 -2.603053 0.577423

H $-3.450542-2.1139690 .239857$

H $2.175705-0.178819-1.471542$

H $2.430868-2.348647-0.254121$

H $3.810243-1.6734000 .645730$

H $3.929871-1.905181-1.092064$

H $4.3797590 .369127-1.385060$

SCF Energy $\left(B 3 L Y P / 6-31 G^{* *}\right)=-689.140535337$

07_c02

MMFF Geometry

O $-0.717029-0.881639-1.050368$

C $0.9640450 .763717-0.504183$

C $-1.402517 \quad 1.428512-0.488955$

C $-0.115997 \quad 1.792149-0.344665$

C $-1.8398080 .002328-0.802792$

C $0.382148-0.619194-0.183664$

C $1.482639-1.605340-0.403122$

C $2.751610-1.232201-0.173838$

C $3.081326 \quad 0.121436 \quad 0.341965$

$\begin{array}{lllll}\text { O } & 2.078004 & 1.045296 & 0.347377\end{array}$

H $0.077421-0.7116690 .865253$
H $1.305867 \quad 0.832365-1.547800$

C $-2.792512-0.621415 \quad 0.259170$

$\begin{array}{llll}C-4.156830 & 0.057762 & 0.306079\end{array}$

$\begin{array}{lllll}O & 4.202322 & 0.367635 & 0.766389\end{array}$

H -2.383359 $0.031553-1.755189$

O $-2.233856-0.593543 \quad 1.578324$

H -2.180079 2.184114 -0.411711

H $0.1654432 .824130-0.161908$

H $1.236079-2.613844-0.715374$

H $3.585114-1.915405-0.284300$

H $-2.933233-1.6806190 .014656$

H $-4.6394210 .050116-0.675755$

H -4.0804621 .0925690 .655451$

H $-4.810472-0.4593201 .017288$

$\mathrm{H}-2.1030520 .337488 \quad 1.829652$

SCF Energy $\left(B 3 L Y P / 6-31 G^{* *}\right)=-689.143108812$

07 c03

MMFF Geometry

O $-0.713446-0.802373-1.090635$

C $0.9809350 .799723-0.454860$

C $-1.3762921 .481384-0.391993$

C $-0.086651 \quad 1.826987-0.231692$

C $-1.8264700 .084084-0.800879$

C $0.383569-0.591400-0.204931$

C $1.472690-1.579446-0.471745$

C $2.746644-1.233342-0.229682$

$\begin{array}{llll}\text { C } 3.095600 & 0.089901 & 0.347295\end{array}$

$\begin{array}{lllll}\text { O } & 2.105873 & 1.025335 & 0.400227\end{array}$

H $0.072914-0.733093 \quad 0.836876$

H $1.3158420 .919993-1.495984$

C $-2.806963-0.6038740 .192860$

$\begin{array}{llll}C & -4.154148 & 0.104887 & 0.278360\end{array}$

$\begin{array}{llll}\text { O } & 4.222540 & 0.299879 & 0.776197\end{array}$

H -2.352607 $0.184682-1.758411$

O $-2.252310-0.6789131 .510773$

H -2.148487 $2.230618-0.242353$

H 0.2010102 .8392220 .031074

H $1.213402-2.568574-0.831286$

H $3.571151-1.920675-0.375382$

H -2.961694-1.637594 -0.139020

H -4.614187 $0.207923-0.708900$

H $-4.058076 \quad 1.0965190 .732349$

H -4.838957 -0.4610630 .919653$

H -2.875047-1.182803 2.062497

SCF Energy $\left(B 3 L Y P / 6-31 G^{* *}\right)=-689.140060499$

07_c04

MM̄FF Geometry

O $0.769212-0.695738 \quad 0.897907$

C -1.0755120 .8006940 .449309$

C $1.2204201 .662193 \quad 0.319162$

C $-0.097754 \quad 1.918544 \quad 0.248126$

C 1.7954250 .2791150 .599984

C $-0.384415-0.5192160 .079536$

C $-1.376916-1.6086120 .317701$

C $-2.683751-1.3511310 .151355$

C $-3.160998-0.025110-0.318419$

O $-2.2483190 .987146-0.348215$

H $-0.100985-0.563617-0.981185$

H -1.3719850 .8279321 .508289$

C $2.716343-0.205881-0.552938$

C $3.202381-1.637624-0.347104$

O $-4.317647 \quad 0.120815-0.689796$

H $2.4029490 .366631 \quad 1.509443$

$\begin{array}{llll}\text { O } & 3.861933 & 0.645735 & -0.679265\end{array}$

H $1.9356842 .469742 \quad 0.177508$

H -0.4705172 .9224840 .073592$

H -1.027185 -2.595116 0.600497

H -3.445429 -2.1104210 .283016$

H $2.177642-0.161910-1.506366$

H $2.372729-2.350587-0.348697$

H $3.745431-1.7336880 .599468$

H $3.901729-1.920156-1.141525$

H 4.4271750 .5072130 .100639

SCF Energy $\left(B 3 L Y P / 6-31 G^{\star *}\right)=-689.141334077$ 
07 c05

MMFF Geometry

O $0.765123-0.7331310 .909700$

C -1.0676600 .7814330 .479811$

C $1.2360991 .635257 \quad 0.406081$

C $-0.0802201 .899438 \quad 0.328573$

C $1.804110 \quad 0.2381190 .636574$

C $-0.380989-0.5333430 .087873$

C $-1.383931-1.6198720 .292350$

C $-2.686190-1.3488860 .110802$

C $-3.144704-0.010161-0.342422$

O $-2.224030 \quad 0.996053-0.334101$

H $-0.091709-0.555765-0.971709$

H $-1.3834980 .782648 \quad 1.533617$

C $2.705541-0.229182-0.539689$

C $3.260886-1.629684-0.300523$

O $-4.2919120 .152260-0.735326$

H 2.4197080 .2913691 .543188

O $3.8237040 .646752-0.715998$

H 1.9505842 .4522230 .340589

H -0.4485462 .9131210 .208140$

H - $1.045310-2.6135190 .563805$

H -3.455894 -2.104020 0.217469

H $2.141125-0.244943-1.479403$

H $2.463921-2.375982-0.233140$

H $3.852995-1.6626670 .620678$

H $3.933634-1.916974-1.116117$

H $3.5022631 .440313-1.177897$

SCF Energy $\left(B 3 L Y P / 6-31 G^{* *}\right)=-689.141466926$

07_c06

MMFF Geometry

O $-0.914445-0.605240-0.856168$

C $1.0237930 .815920-0.453691$

C $-1.2120601 .803939-0.281010$

C $0.117107 \quad 1.977610-0.186564$

C $-1.8546050 .485760-0.672611$

C $0.271975-0.467393-0.077718$

C $1.199212-1.608479-0.340518$

C $2.522568-1.424814-0.213425$

C $3.086663-0.1294470 .241873$

$\begin{array}{lllll}\text { O } & 2.230845 & 0.928943 & 0.306601\end{array}$

H $0.022806-0.4946280 .990118$

H $1.2837220 .848042-1.521753$

C $-3.015208 \quad 0.038744 \quad 0.255017$

C $-2.612637-0.3152301 .679987$

O $4.261122-0.046806 \quad 0.574312$

H -2.292877 $0.630752-1.668608$

O $-3.651376-1.115370-0.311698$

H $-1.8758572 .650160-0.118883$

H 0.5492552 .9458650 .044458

H $0.789610-2.575365-0.611104$

H $3.236066-2.225815-0.366709$

H -3.7704780 .8324090 .292227$

H -2.073256 0.5014912 .167480

H -2.002380 $-1.223487 \quad 1.710411$

H $-3.506519-0.5356872 .274702$

H $-2.930110-1.705604-0.604753$

SCF Energy $\left(B 3 L Y P / 6-31 G^{* *}\right)=-689.142497878$

07_c07

MMFF Geometry

O $-0.731373-0.811440-1.002457$

C $0.985647 \quad 0.811430-0.430473$

C $-1.3659571 .487157-0.296989$

C $-0.074397 \quad 1.829434-0.149813$

C $-1.8262580 .114172-0.768699$

C $0.390977-0.574649-0.154805$

C $1.466796-1.573639-0.431246$

C $2.749483-1.231602-0.234418$

C 3.1247010 .0979180 .309020

$\begin{array}{lllll}O & 2.139145 & 1.035237 & 0.386404\end{array}$

H $0.101867-0.695044 \quad 0.896839$

H $1.2798400 .935118-1.482913$

C $-2.902552-0.579145 \quad 0.113455$

C $-4.162940 \quad 0.2544020 .303608$

$\begin{array}{lllll}O & 4.266275 & 0.309209 & 0.695347\end{array}$
H -2.281989 $0.265331-1.755784$

O $-2.398993-0.917088 \quad 1.408442$

H -2.132219 $2.235552-0.112616$

H 0.2170492 .8321600 .144687

H $1.195655-2.567174-0.770295$

H $3.565633-1.925913-0.395225$

H $-3.182412-1.526143-0.365006$

H $-4.5829490 .568899-0.656368$

H -3.9725531 .1395210 .919255$

H $-4.921393-0.329217 \quad 0.837441$

H $-1.799953-1.6733031 .280259$

SCF Energy $\left(B 3 L Y P / 6-31 G^{* *}\right)=-689.140958769$

$07 \_08$

MM̄MF Geometry

O $-0.881680-0.566726-0.898831$

C $1.0485240 .829862-0.421538$

C $-1.1913041 .803153-0.213053$

C $0.1373721 .976889-0.109121$

C $-1.835540 \quad 0.495809-0.653886$

C $0.298610-0.470775-0.110932$

C $1.230240-1.594475-0.428128$

C $2.552357-1.414949-0.284491$

C $3.108893-0.143206 \quad 0.241148$

$\begin{array}{lllll}\text { O } & 2.250699 & 0.909895 & 0.351239\end{array}$

H $\quad 0.049008-0.552700 \quad 0.953771$

H $1.3158870 .912110-1.485154$

C -2.9944570 .0409690 .280241$

C -2.553895 -0.5374491 .618422$

O $4.280084-0.0752550 .588885$

H -2.285863 $0.686269-1.636793$

O $-3.795947-0.937750-0.395366$

H -1.856063 $2.642611-0.023697$

H 0.5665192 .9373490 .156471

H $0.820418-2.544484-0.753266$

H $3.268358-2.205019-0.476521$

H -3.652009 0.8988830 .466747

H - $-1.917130 \quad 0.1587142 .171390$

H -2.027364 -1.488289 1.486499

H -3.429412 -0.7603462 .238825$

H -4.482398 -1.2413660 .222342$

SCF Energy $\left(B 3 L Y P / 6-31 G^{* *}\right)=-689.136959916$

08_c01

MMFF Geometry

O $0.899847-0.757246-0.135291$

C $-0.971044 \quad 0.711797 \quad 0.272128$

C $1.1640261 .670570-0.480780$

C $-0.094246 \quad 1.882824-0.055360$

C $1.7635630 .282872-0.666281$

C $-0.457410-0.514203-0.496641$

C $-1.329894-1.661871-0.110992$

C -2.616244 -1.429499 0.194895

C -3.203129 -0.0673100 .104172$

O $-2.3360990 .971202-0.066510$

H $-0.533615-0.393727-1.587029$

H -0.9079190 .5690281 .361156$

$\begin{array}{llll}\text { C } & 3.141860 & 0.149038 & 0.026757\end{array}$

C $3.824703-1.171442-0.313501$

$\begin{array}{lllll}\text { O } & -4.415823 & 0.088730 & 0.148081\end{array}$

H $1.8840980 .106060-1.742731$

$\begin{array}{lllll}\text { O } & 2.986928 & 0.200699 & 1.450719\end{array}$

H $1.8012192 .521183-0.711154$

H $-0.4799372 .886566 \quad 0.090655$

H $-0.920509-2.665787-0.106285$

H -3.303921 -2.2261870 .452841$

H $3.7982510 .976096-0.266220$

H $3.978782-1.271816-1.392036$

H $3.240881-2.0278090 .040861$

H $4.798766-1.232647 \quad 0.184127$

H $2.231953-0.3846981 .653438$

SCF Energy $\left(B 3 L Y P / 6-31 G^{\star *}\right)=-689.144350557$

08_c02

MMFF Geometry

O $0.762110 \quad 0.882088-0.003430$

C $-1.027550-0.691173-0.369638$ 
C $1.203629-1.5398150 .225522$

C $-0.062341-1.814981-0.136454$

C $1.730286-0.1254990 .415527$

C -0.5503090 .5313260 .426884$

C $-1.5176551 .633394 \quad 0.149605$

C $-2.8015411 .329643-0.098195$

C -3.292873 -0.071455-0.047745

$\begin{array}{llll}\text { O } & -2.352352 & -1.055761 & 0.026777\end{array}$

H -0.5493650 .3575341 .512644$

H - $1.032491-0.500456-1.453013$

C $3.0233890 .176034-0.380339$

C $4.215041-0.6748410 .032858$

O $-4.493314-0.307478-0.039998$

H 1.9163270 .0467051 .483394

O $3.383631 \quad 1.551361-0.178198$

H $1.887308-2.363048 \quad 0.416415$

H $-0.401675-2.836147-0.278194$

H -1.176438 2.6621650 .175966

H -3.552883 $2.089840-0.276477$

H $2.8388090 .058723-1.454804$

H $4.061416-1.733166-0.194131$

H $4.416187-0.5700201 .104656$

H $5.119324-0.342119-0.488751$

H $2.5524302 .052517-0.297060$

SCF Energy $\left(B 3 L Y P / 6-31 G^{* *}\right)=-689.145895910$

08_c03

MM̄FF Geometry

O $0.713429-1.062827-0.196041$

C -0.9340710 .6296070 .302011$

C $1.3084641 .325058-0.416918$

$\begin{array}{lllll}C & 0.090903 & 1.687709 & 0.024012\end{array}$

C $1.717589-0.124429-0.655770$

C $-0.594354-0.611419-0.536666$

C $-1.618735-1.647253-0.211416$

C $-2.859846-1.2589730 .121042$

C -3.2532620 .1734330 .118390$

O $-2.252903 \quad 1.091311-0.004324$

H $-0.649757-0.420703-1.618228$

H $-0.885123 \quad 0.421441 \quad 1.381150$

C $3.066445-0.499570 \quad 0.007545$

C $3.077684-0.2994731 .520987$

$\begin{array}{lllll}\text { O } & -4.432559 & 0.491651 & 0.190891\end{array}$

H $1.823152-0.255616-1.740946$

O $4.1336930 .253771-0.576550$

H $2.0583752 .083989-0.630044$

H -0.1593772 .7278950 .203775$

H $-1.351478-2.696279-0.270470$

H -3.649932 -1.9678450 .338304$

H $3.265166-1.559045-0.196112$

H $2.267188-0.8503932 .007104$

H 2.9957730 .7601231 .784969

H $4.028495-0.6470811 .940091$

H $4.959336-0.035879-0.150701$

SCF Energy $\left(B 3 L Y P / 6-31 G^{* *}\right)=-689.141908853$

08_c04

MMFF Geometry

O $0.719614-1.065727-0.172737$

C $-0.9326680 .631650 \quad 0.302752$

C $1.3164251 .321254-0.404257$

C $\quad 0.094744 \quad 1.6869140 .022580$

C $1.726320-0.129597-0.625442$

C $-0.586941-0.617065-0.521748$

C $-1.612891-1.650067-0.192875$

C $-2.856709-1.2584020 .125486$

C -3.2503300 .1738650 .102348$

O $-2.248515 \quad 1.090322-0.020558$

H $-0.636859-0.436015-1.605258$

H $-0.893104 \quad 0.433907 \quad 1.384197$

C $3.071672-0.5002320 .049730$

C $3.083860-0.2572761 .555766$

$\begin{array}{lllll}\text { O } & -4.430316 & 0.492773 & 0.157197\end{array}$

H $1.841477-0.268435-1.708701$

O $4.1588390 .227509-0.530482$

H $2.0689512 .080769-0.606221$

H $-0.155641 \quad 2.728150 \quad 0.197049$
H -1.344794 -2.699679-0.235916

H -3.648265 -1.9647910 .345650$

H $3.266601-1.567532-0.113437$

H $2.271403-0.7914592 .057140$

H 3.0063930 .8093791 .791341

H $4.034426-0.5962261 .982647$

H $4.271558-0.093339-1.441895$

SCF Energy $\left(B 3 L Y P / 6-31 G^{* *}\right)=-689.143344615$

08_c05

MMFF Geometry

O $0.714986-1.068020-0.229356$

C -0.9182340 .6225580 .307278$

C $1.3244511 .319385-0.415840$

C $0.110591 \quad 1.6798920 .037320$

C $1.726667-0.130595-0.671285$

C $-0.591151-0.604238-0.556400$

C $-1.619853-1.639618-0.244537$

C $-2.856575-1.250593 \quad 0.103105$

C -3.241292 0.1838120 .130347

O -2.2358921 .0977860 .018300$

H $-0.650030-0.393319-1.633968$

H $-0.8620740 .395993 \quad 1.382296$

C $3.069141-0.524880-0.003350$

C $3.056506-0.4108041 .519662$

$\begin{array}{lllll}0 & -4.417784 & 0.508496 & 0.216847\end{array}$

H $1.838435-0.247168-1.757392$

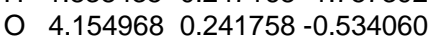

H $2.0610602 .086166-0.641159$

H -0.1410972 .7191470 .221871$

H - $-1.359300-2.689027-0.325180$

H -3.649421 -1.9590350 .311776$

H $3.274278-1.573104-0.252310$

H $2.266389-1.023881 \quad 1.963055$

H $2.9202330 .626347 \quad 1.843605$

H $4.018744-0.7379941 .928751$

H $4.1910571 .086205-0.052986$

SCF Energy $\left(B 3 L Y P / 6-31 G^{* *}\right)=-689.142969969$

08_c06

MMFF Geometry

O $0.869848-0.805977-0.198130$

C $\quad-0.9616920 .705730 \quad 0.250414$

C $1.1598541 .627126-0.569117$

C $-0.0889531 .863072-0.130037$

C $1.7493920 .227565-0.704984$

C $-0.488153-0.533802-0.521694$

C $-1.369948-1.665773-0.109180$

C $-2.642221-1.4139570 .235858$

C $-3.210523-0.0442940 .158450$

O $-2.3352190 .979986-0.046666$

H $-0.589547-0.417409-1.610465$

H $-0.8631560 .577399 \begin{array}{lll}1.338376\end{array}$

C $3.1307730 .111856-0.005972$

C $3.735842-1.279259-0.171041$

$\begin{array}{lllll}\text { O } & -4.419136 & 0.129896 & 0.239842\end{array}$

H $1.8884210 .029596-1.775892$

$\begin{array}{lllll}\text { O } & 3.017131 & 0.395835 & 1.394343\end{array}$

H $1.8009402 .463453-0.834654$

H $-0.4667472 .872554-0.009267$

H $-0.972978-2.674545-0.109044$

H -3.331911-2.199662 0.519749

H $3.8152430 .855168-0.431823$

H $3.819029-1.553568-1.226940$

H $3.139967-2.0378520 .347647$

H $4.736273-1.3137830 .274152$

H 3.8772490 .2111401 .806871

SCF Energy $\left(B 3 L Y P / 6-31 G^{* *}\right)=-689.138856806$

08_c07

MMFF Geometry

O $-0.726590-0.904650-0.048557$

C $1.0484010 .686962-0.349984$

C -1.1889601 .4934010 .278419$

C $0.0741821 .793447-0.074573$

C -1.7080850 .0657310 .416503$

C $0.579901-0.5668710 .399948$ 
C $1.555382-1.6505190 .081421$

C $2.837135-1.329528-0.154350$

C $3.3179450 .072087-0.051245$

$\begin{array}{lllll}\text { O } & 2.370929 & 1.046919 & 0.059051\end{array}$

H $0.576989-0.4347891 .491550$

H $1.0543700 .537519-1.439850$

C -3.022665 - $0.222492-0.358558$

C $-4.126866 \quad 0.803073-0.132957$

O $4.5168420 .316574-0.035234$

H $-1.874230-0.1409701 .481929$

O $-3.541971-1.4935810 .067652$

H -1.8727912 .3043280 .514343$

H $0.4067502 .821787-0.171581$

H $1.216632-2.6804800 .067722$

H $3.592746-2.077777-0.361566$

H $-2.803683-0.310272-1.429701$

H $-3.872248 \quad 1.777977-0.557623$

$\begin{array}{llll}\mathrm{H} & -4.334031 & 0.924487 & 0.935933\end{array}$

H -5.061361 $0.468047-0.596898$

H -4.181054-1.783701-0.605297

SCF Energy $\left(B 3 L Y P / 6-31 G^{* *}\right)=-689.137683999$

Cartesian Coordinates of the most stable conformations found for compounds 1-8 at the PCM/mPW1PW91/6-31+G**//B3LYP/6$31 \mathrm{G}^{*}$ level of theory.

01_c01

B3LYP/6-31G* Geometry

$\begin{array}{lllll}O & 0.863429 & 0.799520 & 0.920278\end{array}$

C $-0.966064-0.793338 \quad 0.768718$

C $0.906070-0.855057-0.868190$

C $-0.203586-1.405587-0.375792$

C $1.4838040 .421346-0.322703$

C $-0.5563340 .675143 \quad 0.975331$

C $-1.256301 \quad 1.569184-0.013714$

C $-2.3999761 .196460-0.595440$

C $-3.064252-0.086654-0.261875$

O $-2.392652-0.946287 \quad 0.560524$

H $-0.814867 \quad 0.998797 \quad 1.991819$

H - $0.785214-1.3581221 .689177$

C $2.987600 \quad 0.3555790 .017779$

C $3.8805160 .058238-1.179043$

O $-4.180699-0.368298-0.630909$

H $1.3375451 .229787-1.060649$

O $3.229652-0.6396410 .999285$

H $1.420603-1.309716-1.710698$

H $-0.610271-2.329493-0.780017$

H $-0.8073412 .542061-0.205072$

H -2.943017 $1.827908-1.290596$

H 3.2435351 .3523010 .415495

H $3.7286240 .792123-1.978442$

H $3.689862-0.944319-1.574374$

H $4.9286120 .098694-0.868385$

H $2.585235-0.4742551 .707888$

SCF Energy $\left(\mathrm{PCM} / \mathrm{mPW} 1 \mathrm{PW} 91 / 6-31+\mathrm{G}^{* *}\right)=-689.024707029$

01_c02

B3LYP/6-31G* Geometry

O $-0.410805-0.676305-0.808365$

C 0.9042680 .6466230 .796603

C $-1.2528901 .491876-0.074865$

C -0.1265331 .7192610 .600999$

C $-1.5442110 .191415-0.778143$

C $0.349447-0.7232480 .404361$

C $1.458831-1.7121600 .190183$

C $2.712580-1.302460-0.025924$

C $3.0658520 .130091-0.182830$

$\begin{array}{llll}\text { O } & 2.090393 & 1.045503 & 0.060275\end{array}$

H $-0.296445-1.0559141 .226655$

H $1.1928580 .596083 \quad 1.856418$

C $-2.800760-0.545019-0.265527$

C $-4.1018600 .202656-0.566959$

$\begin{array}{llllll}\text { O } & 4.151987 & 0.497702 & -0.572372\end{array}$

H $-1.7350460 .414118-1.837633$

O $-2.640471-0.7518101 .140884$

H $-1.9997302 .276210-0.173697$

H $0.0800712 .691807 \quad 1.040001$
H $1.202155-2.7689020 .191380$

H $3.534989-1.991550-0.188164$

H -2.808701-1.513874-0.788667

H $-4.2211110 .384456-1.641423$

H $-4.134667 \quad 1.161553-0.039985$

H $-4.964289-0.389074-0.236253$

H -3.367263 -1.319844 1.440596

SCF Energy $\left(\mathrm{PCM} / \mathrm{mPW} 1 \mathrm{PW} 91 / 6-31+\mathrm{G}^{\star \star}\right)=-689.023126124$

01_c03

B3LYP/6-31G* Geometry

O $-0.413037-0.709986-0.837444$

C $0.8740540 .642652 \quad 0.769183$

C $-1.2589521 .477299-0.186021$

C $-0.153057 \quad 1.7121170 .524491$

C $-1.5584590 .142310-0.819764$

C $0.341176-0.7384120 .379115$

C $1.464871-1.713923 \quad 0.181705$

C $2.718909-1.292807-0.009621$

C $3.063123 \quad 0.141851-0.157834$

O 2.0718681 .0496380 .061574

H $-0.304699-1.0792701 .198335$

H $1.1332980 .608273 \quad 1.837367$

C $-2.790464-0.566566-0.196067$

C $-4.1135280 .127478-0.530685$

O $4.1532620 .522756-0.520258$

H - $-1.7849210 .305434-1.882317$

O $-2.645917-0.7505411 .214024$

H -1.983002 $2.273390-0.348210$

H 0.0606782 .7002390 .924415

H $1.216861-2.7725270 .177775$

H $3.550022-1.974812-0.156053$

H -2.798059-1.581859-0.605909

H -4.269681 $0.196229-1.613510$

H $-4.151181 \quad 1.143701-0.118326$

H $-4.943049-0.439144-0.097486$

H -2.581709 0.1334201 .613731

SCF Energy $\left(\mathrm{PCM} / \mathrm{mPW} 1 \mathrm{PW} 91 / 6-31+\mathrm{G}^{* *}\right)=-689.023488708$

01_c04

B3LYP/6-31G* Geometry

O 1.0134200 .6221340 .685359

C $-0.933566-0.8267770 .730129$

C $0.746977-1.030339-1.090592$

C $-0.345009-1.497297-0.483973$

C $1.4729350 .191209-0.606294$

C -0.3975530 .6090840 .879445$

C $-1.1259681 .548034-0.046241$

C $-2.348136 \quad 1.259288-0.502830$

C $-3.0684180 .032187-0.085605$

O $-2.380619-0.872572 \quad 0.673965$

H $-0.531956 \quad 0.9523091 .913257$

H $-0.697255-1.4007651 .631870$

C $2.9988700 .010545-0.460763$

C $3.393827-1.113798 \quad 0.498880$

O $-4.234952-0.166933-0.332875$

H $1.3223391 .012561-1.327421$

O $3.5783821 .252273-0.079970$

H $1.139478-1.511361-1.984805$

H $-0.865954-2.382210-0.841509$

H $-0.6267552 .481887-0.297816$

H $-2.9109061 .925441-1.148103$

H $3.398215-0.202649-1.459927$

H $3.026201-2.0861240 .152373$

H $2.981195-0.9305541 .496444$

H $4.484010-1.1588030 .576139$

H 3.1077891 .5282340 .724737

SCF Energy $\left(\mathrm{PCM} / \mathrm{mPW} 1 \mathrm{PW} 91 / 6-31+\mathrm{G}^{* *}\right)=-689.024388721$

$01 \_05$

B3LYP/6-31G* Geometry

$\begin{array}{lllll}O & 0.476861 & 0.693467 & -0.484302\end{array}$

C - $-1.026606-0.833634 \quad 0.727925$

C $1.141247-1.636332-0.155044$

C $-0.039817-1.9148990 .399251$

C $1.548243-0.248770-0.572437$

C $-0.365503 \quad 0.5442850 .658873$ 
C $-1.397377 \quad 1.634603 \quad 0.595321$

$\begin{array}{llll}C & -2.649578 & 1.366747 & 0.213118\end{array}$

C -3.065932 $0.025819-0.269306$

O $-2.163739-0.985779-0.159821$

H 0.2257690 .6565421 .582269

H -1.404378 $-0.968017 \quad 1.751977$

$\begin{array}{llll}\text { C } 2.801361 & 0.264527 & 0.175894\end{array}$

C $3.2568321 .627945-0.336802$

O $-4.134713-0.174450-0.801011$

H $1.807586-0.275678-1.639630$

O $3.797286-0.737829-0.032432$

H $1.869293-2.418409-0.347080$

H $-0.323438-2.9363990 .638417$

H -1.0843642 .6463800 .841661$

H -3.4179402 .1301440 .148150$

H $2.5595600 .336572 \quad 1.249745$

H $2.4611452 .370599-0.229777$

H $3.5316381 .561264-1.395612$

H 4.1319061 .9802910 .224224

H $4.620446-0.4283030 .375438$

SCF Energy $\left(P C M / m P W 1 P W 91 / 6-31+G^{\star *}\right)=-689.022949193$

01 c06

B3LYP/6-31G* Geometry

O $0.4739870 .687356-0.471928$

C $-1.035784-0.8309360 .741335$

C $1.139333-1.639116-0.115961$

C $-0.043229-1.9141950 .436802$

C $1.544960-0.257505-0.552617$

C $-0.3730450 .545751 \quad 0.669233$

C $-1.404901 \quad 1.6355180 .593092$

C -2.6520131 .3652860 .196002$

C $-3.0600630 .022891-0.289559$

O $-2.159555-0.988636-0.161768$

H $\quad 0.2141320 .6632201 .594479$

H $-1.427308-0.9571221 .761133$

C 2.8182350 .2509730 .177716

C $3.2316281 .645764-0.282477$

O $-4.119554-0.179542-0.838505$

H $1.788330-0.296090-1.626064$

O $3.873598-0.6970440 .010397$

H $1.875142-2.420594-0.279993$

H $-0.319928-2.9322050 .697710$

H -1.096335 $2.647910 \quad 0.842347$

H -3.420684 2.1273680 .120354

H 2.6253270 .2592981 .256857

H $2.4281072 .369157-0.120289$

H $3.4615821 .644994-1.357386$

H $4.124326 \quad 1.972007 \quad 0.260212$

H $4.208783-0.601601-0.896323$

SCF Energy $\left(\mathrm{PCM} / \mathrm{mPW} 1 \mathrm{PW} 91 / 6-31+\mathrm{G}^{* *}\right)=-689.022752776$

01_c08

B3LYP/6-31G* Geometry

O $-0.473315-0.711892-0.508959$

C 1.0135490 .8326780 .705260

C $-1.1405341 .617197-0.229055$

C $0.034414 \quad 1.907916 \quad 0.333079$

C $-1.5572850 .218641-0.600809$

C $0.359471-0.5499230 .638558$

C $1.396758-1.6356160 .594055$

C $2.655081-1.3651120 .234394$

C $3.077780-0.024747-0.241966$

O $2.1686470 .984904-0.155986$

H $-0.237954-0.658951 \quad 1.558393$

H 1.3638660 .9836591 .736869

C $-2.797865-0.2870350 .186829$

C $-3.326099-1.596546-0.379319$

O $4.1567170 .180239-0.749714$

H $-1.833620 \quad 0.219870-1.663704$

$\begin{array}{llll}\text { O } & -3.858009 & 0.666970 & 0.102488\end{array}$

$\mathrm{H}-1.8398562 .410022-0.484098$

H 0.3286042 .9355050 .530270

H $1.081536-2.6476630 .835886$

H $3.426044-2.1269730 .184884$

H $-2.505444-0.4341961 .239078$

H -2.533631-2.349414 -0.406230
H -3.694671 -1.439676 -1.399492

H $-4.155721-1.966850 \quad 0.230323$

H -3.6430881 .4081840 .689037$

SCF Energy $\left(\mathrm{PCM} / \mathrm{mPW} 1 \mathrm{PW} 91 / 6-31+\mathrm{G}^{\star \star}\right)=-689.022375064$

01_c09

B3LYP/6-31G* Geometry

O $-0.906286-0.8387790 .716342$

C $1.0913510 .504017 \quad 1.045863$

C $-0.771704 \quad 1.342783-0.369935$

C $0.396694 \quad 1.5639110 .233000$

C $-1.4851760 .022542-0.269222$

C $0.516651-0.888790 \quad 0.731563$

C $1.101092-1.428395-0.547840$

C $2.277256-0.993551-1.009301$

C $3.089109-0.000559-0.265473$

$\begin{array}{lllll}\text { O } & 2.525597 & 0.570144 & 0.839834\end{array}$

H $0.751376-1.5830361 .548747$

H 0.9819050 .7115952 .115210

C -2.9659440 .1857690 .119661$

C $-3.689792-1.152798 \quad 0.244168$

$\begin{array}{lllll}\text { O } & 4.230827 & 0.271697 & -0.557534\end{array}$

H - $1.463462-0.472383-1.256423$

O $-3.531863 \quad 0.994392-0.913302$

H -1.260218 $2.105217-0.969147$

H 0.9038782 .5234630 .163798

H $\quad 0.539176-2.208516-1.058104$

H $2.738395-1.380765-1.911723$

H -2.998129 $0.715137 \quad 1.085077$

H -3.229652 -1.7756781 .015759$

H -3.660450 -1.690608 -0.710261

H $-4.741409-0.9938150 .514815$

H $-4.4648371 .140193-0.695374$

SCF Energy $\left(\mathrm{PCM} / \mathrm{mPW} 1 \mathrm{PW} 91 / 6-31+\mathrm{G}^{* *}\right)=-689.022966411$

01_c10

B3LYP/6-31G* Geometry

O $-0.581608-0.635219-0.496732$

C $0.9689850 .867800 \quad 0.706162$

C -1.100932 $1.761711-0.300706$

C $\quad 0.046508 \quad 1.992748 \quad 0.339020$

C $-1.5318180 .407608-0.778871$

C $0.237804-0.4742120 .666418$

C $1.217761-1.6145620 .668896$

C $2.487230-1.4222440 .298082$

C $2.973067-0.124053-0.233350$

$\begin{array}{llll}\text { O } & 2.118547 & 0.934572 & -0.174075\end{array}$

H $-0.379405-0.520327 \quad 1.575466$

H $1.341414 \quad 1.0057051 .731316$

C $-2.949462-0.028734-0.334198$

C $-3.169038-0.103212 \quad 1.179521$

$\begin{array}{lrrrr}O & 4.054174 & 0.004683 & -0.760666\end{array}$

H $-1.5695640 .433728-1.877026$

O $-3.253674-1.270152-0.956107$

H $-1.7702732 .584077-0.547364$

H 0.3497953 .0004350 .609234

H $0.854496-2.5988830 .954418$

H $3.219736-2.2224480 .279368$

H -3.655733 $0.697036-0.758401$

H -2.953251 $0.852119 \quad 1.671586$

H -2.536920 - 0.8768421 .630085

H $-4.210134-0.3726621 .380788$

H $-2.470301-1.830112-0.814672$

SCF Energy $\left(P C M / m P W 1 P W 91 / 6-31+G^{\star \star}\right)=-689.022570517$

$01 \mathrm{c} 11$

B3LYP/6-31G* Geometry

O $-0.904869-0.8361810 .723935$

C 1.0972240 .5047291 .047480

C $-0.7703861 .345420-0.360620$

C 0.3994841 .5661370 .239352

C $-1.4837340 .026068-0.260230$

C $0.519314-0.888140 \quad 0.739145$

C $1.099928-1.434449-0.538966$

C $2.273334-1.000638-1.008344$

C $3.087790-0.002971-0.273417$

$\begin{array}{lllll}\text { O } & 2.530415 & 0.569510 & 0.833779\end{array}$ 
H $0.753111-1.579591 \quad 1.558868$

H 0.9936640 .7143682 .116988

C -2.9733520 .1996410 .116499$

C $-3.699981-1.1328340 .274127$

O $4.226916 \quad 0.270216-0.574243$

H - $-1.452238-0.474321-1.248075$

O $-3.6105241 .040179-0.844932$

H - $-1.2668342 .112641-0.947573$

H 0.9023402 .5283510 .176782

H $\quad 0.537715-2.219286-1.041762$

H $2.731324-1.392738-1.910299$

H $-3.0167790 .758427 \quad 1.057464$

H $-3.236573-1.743337 \quad 1.053228$

H -3.668556 -1.705253 -0.663952

H $-4.748395-0.9554950 .533459$

H -3.761997 $0.508677-1.643595$

SCF Energy $\left(P C M / m P W 1 P W 91 / 6-31+G^{* *}\right)=-689.022920788$

01_c12

B3LYP/6-31G* Geometry

O $-0.898685-0.8488590 .715984$

C $1.0801330 .524509 \quad 1.024991$

C $-0.776503 \quad 1.297710-0.434315$

C $0.386878 \quad 1.5497110 .166904$

C $-1.495956-0.013444-0.282354$

C $0.523953-0.882407 \quad 0.741595$

C $1.126340-1.445608-0.519094$

C $2.303625-1.010429-0.977797$

C $3.0972270 .008469-0.249965$

$\begin{array}{lllll}\text { O } & 2.514427 & 0.602420 & 0.834721\end{array}$

H $0.761144-1.5527771 .577715$

H 0.9515260 .7624682 .085748

$\begin{array}{llll}\text { C }-2.976384 & 0.156726 & 0.139379\end{array}$

C $-3.717304-1.1726800 .164170$

O $4.238848 \quad 0.286838-0.535086$

H $-1.492756-0.532343-1.257220$

O $-3.6530951 .003254-0.791054$

H $-1.2331282 .029459-1.096347$

$\begin{array}{llll}\mathrm{H} & 0.899021 & 2.501878 & 0.049171\end{array}$

H $0.576914-2.242406-1.016899$

H $2.778361-1.414123-1.865804$

H -2.9805860 .6014661 .145484$

H $-3.216884-1.8804130 .829883$

H -3.753144 -1.600366 - 0.844538

H $-4.745353-1.0207600 .506171$

H -3.469125 $1.923337-0.550968$

SCF Energy $\left(\mathrm{PCM} / \mathrm{mPW} 1 \mathrm{PW} 91 / 6-31+\mathrm{G}^{* *}\right)=-689.022250364$

$01 \mathrm{c} 13$

B3LYP/6-31G* Geometry

O $0.998817 \quad 0.521783 \quad 0.780456$

C $-0.973929-0.890104 \quad 0.673877$

C $0.735806-0.969770-1.127707$

C $-0.371979-1.469628-0.579427$

C $1.4641480 .206734-0.534313$

C -0.4135470 .5173780 .948002$

C -1.1125521 .5434220 .094556$

C $-2.3303901 .315332-0.406467$

C $-3.0779770 .070617-0.107861$

O $-2.420662-0.9073180 .584595$

H -0.5595930 .7781692 .004191$

H $-0.765452-1.539981 \quad 1.529767$

C $2.982959-0.018174-0.456468$

C $3.391438-1.032308 \quad 0.611006$

O $-4.242726-0.086919-0.393006$

H $1.3195141 .083085-1.189940$

O $3.5617191 .263382-0.225592$

H $1.135552-1.382503-2.052272$

H $-0.897579-2.314672-1.017828$

H $-0.5933892 .486377-0.066211$

H -2.871046 2.043805-1.001459

H $3.289593-0.392853-1.449034$

H $2.924559-2.0065510 .429144$

H $3.091328-0.6783021 .600104$

H $4.479102-1.1776740 .601916$

H $4.5063681 .132675-0.051196$

SCF Energy $\left(P C M / m P W 1 P W 91 / 6-31+G^{* *}\right)=-689.021621489$ 02_c02

B3LYP/6-31G* Geometry

$\begin{array}{llll}O & -1.022086 & 0.560317 & 0.709487\end{array}$

C $0.966129-0.8413620 .716801$

C $-0.728280-1.059634-1.087545$

C $0.379707-1.508576-0.498860$

C $-1.4765920 .146282-0.590022$

$\begin{array}{llll}\text { C } & 0.391911 & 0.574908 & 0.898559\end{array}$

C $1.0869481 .554196-0.010109$

C $2.3108871 .308790-0.486471$

C $3.0680610 .092190-0.104696$

$\begin{array}{llll}\text { O } & 2.414106 & -0.844796 & 0.643798\end{array}$

H $0.5202530 .901367 \quad 1.938573$

H $0.759755-1.4385161 .611062$

C $-2.989509-0.113352-0.430186$

C $-3.766648 \quad 1.161119-0.090029$

O $4.236534-0.069343-0.370108$

H $-1.3400930 .976716-1.304895$

O $-3.208619-1.1353910 .531516$

H -1.130514 -1.551501-1.970832

H $\quad 0.907495-2.386024-0.864385$

H $\quad 0.5611472 .480871-0.232494$

H $2.8490082 .004888-1.121079$

H -3.357008 -0.518764-1.380965

H -3.679483 $1.906828-0.889748$

H -3.390808 1.6078400 .836172

H -4.8246750 .9181690 .044326$

H -2.690251 -0.8755411 .312078$

SCF Energy $\left(\mathrm{PCM} / \mathrm{mPW} 1 \mathrm{PW} 91 / 6-31+\mathrm{G}^{* *}\right)=-689.024299638$

$02 \_$c03

B3LYP/6-31G* Geometry

O $0.570867-0.575473-0.651556$

C $\quad-0.9455780 .839794 \quad 0.673158$

C $1.0943551 .789758-0.350069$

C $-\begin{array}{lllll}0.064087 & 1.988126 & 0.278307\end{array}$

C $1.5672650 .434154-0.804840$

C $-0.198586-0.488908 \quad 0.554977$

C - $1.157040-1.6449420 .548990$

C $-2.448728-1.463410 \quad 0.257236$

C $-2.987950-0.153481-0.185245$

O $-2.1528990 .918071-0.129528$

H $0.472243-0.5602461 .420131$

H -1.255476 0.9520121 .722112

C $2.9237120 .031017-0.186477$

C $3.400602-1.331784-0.684737$

O $-4.102087-0.025275-0.642082$

H $1.735003 \quad 0.483402-1.890694$

$\begin{array}{lllll}\text { O } & 2.773198 & 0.049488 & 1.234897\end{array}$

H $1.7450242 .628750-0.589657$

H -0.4021542 .9880530 .536870$

H $-0.760216-2.6342650 .764471$

H $-3.166131-2.277357 \quad 0.239688$

H $3.6509830 .803417-0.493964$

H $3.545001-1.325376-1.771074$

H $2.666085-2.102877-0.438133$

H $\quad 4.361590-1.595772-0.225186$

H $3.591630-0.2961721 .623784$

SCF Energy $\left(\mathrm{PCM} / \mathrm{mPW} 1 \mathrm{PW} 91 / 6-31+\mathrm{G}^{* *}\right)=-689.023426881$

$02 \_\mathrm{c} 04$

B3LYP/6-31G* Geometry

O $0.571383-0.595266-0.719627$

C $-0.902308 \quad 0.811134 \quad 0.666433$

C $1.103366 \quad 1.763696-0.435554$

C $-0.026863 \quad 1.958950 \quad 0.247987$

C $1.5843120 .399678-0.859171$

C $-0.180083-0.5280720 .498033$

C $-1.157413-1.6675620 .479456$

C $-2.454410-1.4588860 .232999$

C $-2.988766-0.128883-0.147703$

O $-2.1336980 .929713-0.088673$

H $0.501723-0.6390821 .351011$

H -1.1701540 .9110591 .728331$

C $2.9168400 .018589-0.159651$

C $3.393720-1.376401-0.536504$ 
O $-4.1150420 .033901-0.559324$

H $1.7912060 .425618-1.938331$

$\begin{array}{lllll}\text { O } & 2.805375 & 0.054077 & 1.264742\end{array}$

H $1.7273312 .609014-0.722766$

H -0.3698872 .9594150 .499357$

H $-0.768035-2.6683130 .649962$

H -3.183035 -2.262553 0.210275

H $3.6676000 .757201-0.493340$

H $3.571554-1.444399-1.615121$

H $2.642628-2.122136-0.263479$

H $4.326380-1.604333-0.012357$

H 2.5081740 .9456601 .511567

SCF Energy (PCM/mPW1PW91/6-31+G** $)=-689.023969845$

\section{2_c05}

B3LYP/6-31G* Geometry

O $-0.899366-0.859792-0.676502$

C $1.083114 \quad 0.483157-1.049265$

$\begin{array}{llll}\text { C } & -0.780853 & 1.344840 & 0.355831\end{array}$

C $0.3850111 .556785-0.256626$

C -1.4996250 .0282020 .283964$

C $0.523797-0.905550-0.692956$

C $1.114087-1.4015400 .600652$

C $2.288621-0.9430231 .043278$

C 3.0891500 .0344870 .267293

O $2.5159740 .568906-0.853065$

H $0.761899-1.621023-1.490467$

H $0.961966 \quad 0.661871-2.122432$

C $-2.9747970 .128128-0.159709$

C -3.8533180 .8833090 .829049$

$\begin{array}{lllll}\text { O } & 4.228724 & 0.326079 & 0.546530\end{array}$

H $-1.486965-0.456523 \quad 1.275515$

O $-3.512956-1.180512-0.281401$

H $-1.239795 \quad 2.1268210 .955735$

H $0.8938592 .516037-0.199355$

H $\quad 0.560938-2.170613 \quad 1.136430$

H $2.756638-1.299221 \quad 1.954820$

H $-2.987150 \quad 0.640877-1.136065$

H -3.5794471 .9411050 .890805$

H -3.7858340 .4378801 .827967$

H -4.8950370 .8186390 .501576$

H $-2.871208-1.686069-0.808098$

SCF Energy $\left(\mathrm{PCM} / \mathrm{mPW} 1 \mathrm{PW} 91 / 6-31+\mathrm{G}^{* *}\right)=-689.025466251$

\section{2 c06}

B3LYP/6-31G* Geometry

O $0.590920-0.579792-0.510105$

C $-1.007903 \quad 0.855610 \quad 0.708112$

C $1.0698801 .809424-0.215384$

C $-0.1044092 .007173 \quad 0.383213$

C $1.5447560 .471590-0.709615$

C $-0.240668-0.4641950 .650843$

C $-1.190230-1.6289070 .622680$

C $-2.459337-1.462557 \quad 0.237968$

C $-2.970230-0.168354-0.280627$

O $-2.146857 \quad 0.910711-0.188854$

H $0.376541-0.5006561 .558961$

H $-1.4002300 .962741 \quad 1.729455$

C $2.9352120 .063732-0.159850$

C $3.535884-1.105457-0.938895$

O $-4.045981-0.061202-0.824894$

H $1.6435880 .547646-1.803372$

O $2.874381-0.2239591 .238115$

H $1.7347492 .647881-0.415298$

H $-0.435514 \quad 3.0030890 .664307$

H $-0.807064-2.6097800 .894379$

H -3.171397-2.280224 0.195889

H $3.5972630 .935075-0.244675$

H $3.698135-0.843053-1.990268$

H $2.858554-1.967743-0.912188$

H $4.496875-1.394343-0.502130$

H $2.551074-1.1350571 .320160$

SCF Energy $\left(\mathrm{PCM} / \mathrm{mPW} 1 \mathrm{PW} 91 / 6-31+\mathrm{G}^{* *}\right)=-689.021739035$

02 C07

B3LYP/6-31G* Geometry

O $\begin{array}{llll}0.860991 & 0.703590 & 1.021694\end{array}$
C $0.950309-0.8795810 .670752$

C $-0.911348-0.716475-0.966709$

C $0.192681-1.336120-0.548381$

C $-1.4738370 .480608-0.252253$

C $0.554898 \quad 0.557671 \quad 1.055098$

C 1.2697331 .5553950 .181031

C $2.4151091 .243622-0.432632$

C $3.066023-0.075342-0.246297$

O $2.377901-1.0209860 .459430$

H 0.8201350 .7502232 .102697

H $0.753501-1.5502211 .513396$

C $-2.9871990 .378877 \quad 0.018355$

C -3.379628 -0.8395610 .849473$

O $4.185272-0.321399-0.632992$

H $-1.3321021 .368055-0.892644$

O $-3.5915200 .371119-1.275326$

H $-1.445482-1.035509-1.857452$

H $\quad 0.598635-2.200279-1.069192$

H 0.8285912 .5471490 .100231

H $2.9681001 .946844-1.046298$

H -3.2664581 .2931710 .565618$

H -2.860868 -0.8260221 .812551$

H -3.126622 -1.7672970 .326143$

H -4.459119-0.837283 1.047379

H -4.544050 $0.238014-1.155495$

SCF Energy $\left(\mathrm{PCM} / \mathrm{mPW} 1 \mathrm{PW} 91 / 6-31+\mathrm{G}^{\star \star}\right)=-689.022685016$

02_c08

B3LYP/6-31G* Geometry

O $-0.8585330 .677632 \quad 1.044376$

C $0.953990-0.8964360 .650743$

C $-0.913639-0.697658-0.974893$

C $0.192720-1.325563-0.575979$

C $-1.4763470 .479096-0.229869$

C $0.558426 \quad 0.529861 \quad 1.073278$

C 1.2714931 .5515390 .226204

C $2.4143081 .256672-0.400472$

C $3.065471-0.067323-0.253625$

O $2.381160-1.0309170 .430732$

H $\quad 0.8238420 .6944772 .125509$

H $0.760563-1.5886311 .476508$

C $-2.9974090 .365622 \quad 0.033407$

C $-3.387600-0.881750 \quad 0.811412$

O $4.182378-0.302317-0.653500$

H $-1.3259811 .385620-0.848258$

O $-3.6956680 .330578-1.210797$

H $-1.456567-1.002626-1.865555$

H $0.594158-2.180586-1.114839$

H 0.8323422 .5463240 .176978

H $2.966374 \quad 1.977038-0.994830$

H -3.2835691 .2561310 .614199$

H $-2.846050-0.9171921 .760608$

H -3.153846 - 1.7843430 .237882

H $-4.462715-0.8721001 .013767$

H -3.611726 $1.201721-1.629694$

SCF Energy $\left(\mathrm{PCM} / \mathrm{mPW} 1 \mathrm{PW} 91 / 6-31+\mathrm{G}^{\star *}\right)=-689.022716807$

02 c09

B3LYP/6-31G* Geometry

O -0.8606830 .7645540 .970827$

C $0.944770-0.8402910 .711590$

C $-0.904034-0.746319-0.945173$

C $0.194221-1.350923-0.490730$

C $-1.4773230 .478195-0.289245$

C $0.554410 \quad 0.616744 \quad 1.019036$

C 1.2784731 .5640140 .098058

C $2.4258451 .215216-0.491594$

C $3.068860-0.095574-0.234182$

O $2.371416-1.0012580 .515502$

H 0.8154650 .8621922 .056525

H $0.736778-1.4668061 .584878$

C -2.998579 $0.385163-0.013196$

C -3.380401-0.743735 0.938464

O $4.188091-0.368152-0.601827$

H $-1.3412301 .333829-0.972468$

O $-3.6972030 .310146-1.254898$

H -1.408217 -1.099711-1.841962 
H $0.607346-2.232877-0.974750$

H $0.8429232 .552294-0.036898$

H $2.9859691 .882932-1.137588$

H -3.2909421 .3437870 .429755$

H $-2.856009-0.636393 \quad 1.891858$

H -3.113456 - 1.7224080 .517973

H $-4.459002-0.7280011 .124786$

H -3.770049-0.626092 -1.497114

SCF Energy $\left(\mathrm{PCM} / \mathrm{mPW} 1 \mathrm{PW} 91 / 6-31+\mathrm{G}^{\star *}\right)=-689.021929257$

02 C17

B3LYP/6-31G* Geometry

O $0.481306-0.673267-0.529315$

C $-1.0015840 .828120 \quad 0.738728$

C $1.1551491 .645769-0.162199$

C $-0.019366 \quad 1.9145540 .411082$

C $1.5719880 .266144-0.584647$

C $-0.351733-0.552394 \quad 0.628994$

C $-1.390817-1.6340670 .549422$

C $-2.646205-1.349127 \quad 0.190643$

C $-3.0615830 .006240-0.249745$

O $-2.151195 \quad 1.010528-0.124597$

H $\quad 0.251168-0.6922321 .540037$

H $-1.3578350 .942826 \quad 1.772714$

C $2.782133-0.319116 \quad 0.182014$

C $4.0618990 .484810-0.010438$

$\begin{array}{llll}\text { O } & -4.135281 & 0.228046 & -0.760962\end{array}$

H $1.8479440 .284688-1.647434$

O $3.025336-1.634932-0.291918$

H $1.8571642 .448624-0.374420$

H -0.3095682 .9336090 .652084$

H $-1.082705-2.6534550 .769446$

H -3.419916 -2.106268 0.117626

H $2.531761-0.340587 \quad 1.257842$

H 3.9906731 .4736420 .454203

H $4.2861540 .605633-1.076460$

H $4.897105-0.051368 \quad 0.449880$

H $2.147442-2.033713-0.424319$

SCF Energy $\left(\mathrm{PCM} / \mathrm{mPW} 1 \mathrm{PW} 91 / 6-31+\mathrm{G}^{* *}\right)=-689.026201736$

\section{3 c01}

B3LYP/6-31G* Geometry

O $-0.507211-0.967880-0.342246$

C $1.0219850 .874554-0.868324$

C $-1.4195891 .254342-0.683370$

C $-0.1867931 .758847-0.763405$

C $-1.700804-0.224814-0.626945$

C $0.610627-0.574733-1.135302$

C $1.738531-1.522832-0.844272$

C $2.756485-1.150339-0.062381$

C 2.7817980 .1552560 .644100

$\begin{array}{lllll}\text { O } & 1.795649 & 1.045371 & 0.347750\end{array}$

H $\quad 0.336290-0.642273-2.201803$

H $1.6612981 .203460-1.700227$

C $-2.678255-0.630547 \quad 0.495091$

C $-4.089214-0.086578 \quad 0.313757$

O 3.5924720 .4182361 .502921

H -2.117266 - $0.565865-1.593198$

O $-2.198707-0.1842651 .752455$

H $-2.2791911 .918715-0.650261$

H $-0.0154002 .831984-0.759311$

H $1.683907-2.523161-1.266537$

H $3.584686-1.8099770 .174782$

H -2.715202 -1.7327800 .467448$

H $-4.517546-0.408906-0.641856$

H -4.1032251 .0069640 .360111$

H $-4.726289-0.459708 \quad 1.121013$

H $-1.258318-0.4290661 .789105$

SCF Energy $\left(\mathrm{PCM} / \mathrm{mPW} 1 \mathrm{PW} 91 / 6-31+\mathrm{G}^{* *}\right)=-689.026932319$

03_c02

B3LYP/6-31G* Geometry

O $-0.694656-0.653691-0.503006$

C $1.0685691 .032946-0.715328$

C $-1.3020961 .700230-0.461171$

C $-0.0126752 .044384-0.461358$

C $-1.7704070 .282390-0.640425$
C $0.475449-0.283317-1.226489$

C $1.467448-1.404998-1.108113$

C $2.504918-1.310212-0.270940$

C $2.680718-0.1615740 .653381$

$\begin{array}{lllll}O & 1.831932 & 0.892452 & 0.509704\end{array}$

H $\quad 0.213292-0.130159-2.287300$

H $1.7622091 .409010-1.481284$

C -2.838399 -0.1585890 .383497$

C $-2.374637-0.0316891 .836082$

O $3.494302-0.1616121 .548904$

H -2.219220 $0.161336-1.643020$

O $-3.251856-1.484108 \quad 0.076317$

H -2.075278 $2.455330-0.329066$

H $0.2995703 .071264-0.290859$

H $1.297753-2.299706-1.702143$

H $3.236418-2.102882-0.152915$

H -3.725292 0.4666630 .222687

H -2.170739 1.0107062 .104602

H $-1.456044-0.6046451 .998100$

H -3.153153 -0.418765 2.499881

H $-2.440621-2.0199020 .071161$

SCF Energy $\left(\mathrm{PCM} / \mathrm{mPW} 1 \mathrm{PW} 91 / 6-31+\mathrm{G}^{* *}\right)=-689.026676313$

03_c03

B3LYP/6-31G* Geometry

O $-0.543451-0.741047-0.310838$

C $1.1407381 .007317-0.669717$

C $-1.2400611 .586000-0.306146$

C $0.0336751 .977270-0.374096$

C $-1.6548850 .143450-0.441808$

C $0.574191-0.348817-1.101217$

C $1.612711-1.428919-0.997576$

C $2.696737-1.262570-0.233956$

C $2.887400-0.071027 \quad 0.630827$

O 1.9908260 .9429650 .504940

H $\quad 0.256145-0.243494-2.153155$

H $1.7664401 .386430-1.490872$

C $-2.673579-0.267301 \quad 0.636167$

C $-3.070132-1.737948 \quad 0.534702$

O $3.758569-0.0050201 .468821$

H $-2.139040-0.009652-1.425034$

O -3.7955190 .5946720 .432019$

H -2.043339 $2.296909-0.136518$

H $0.3144593 .017088-0.227590$

H $1.438068-2.354681-1.540406$

H $\quad 3.462572-2.024337-0.130817$

H $-2.212158-0.0744131 .617084$

H -2.199873 -2.386401 0.665651

H $-3.522582-1.943043-0.442232$

H -3.804132 -1.9887121 .310927$

H -4.440729 0.4070221 .130346

SCF Energy $\left(\mathrm{PCM} / \mathrm{mPW} 1 \mathrm{PW} 91 / 6-31+\mathrm{G}^{* *}\right)=-689.025374330$

03_c04

B3LYP/6-31G* Geometry

O $-0.532966-0.763545-0.316564$

C $1.1438930 .982064-0.708864$

C $-1.2439191 .555535-0.397943$

C $0.0268691 .956646-0.469786$

C $-1.6532960 .108136-0.465274$

C $0.589832-0.388458-1.108202$

C $1.634159-1.457704-0.958437$

C $2.701844-1.263408-0.178418$

C $2.867991-0.0479470 .657257$

$\begin{array}{lllll}\text { O } & 1.968091 & 0.958215 & 0.484275\end{array}$

H $0.283650-0.316451-2.166165$

H $1.7833871 .339314-1.529128$

C $-2.655203-0.281507 \quad 0.649405$

C -3.142381-1.715531 0.494681

O $3.7202260 .047893 \quad 1.511053$

H -2.150835 - $0.081292-1.435186$

$\begin{array}{llll}\text { O } & -3.800290 & 0.572519 & 0.588806\end{array}$

H -2.048602 $2.280216-0.301428$

H $0.2951863 .006172-0.380306$

H $1.476256-2.398720-1.479504$

H $3.470370-2.016719-0.039386$

H -2.134830 -0.168584 1.610925 
H $-2.298324-2.4097850 .482683$

H $-3.701885-1.822180-0.442008$

H $-3.810570-1.9743881 .321394$

H -3.6230241 .3548051 .131034$

SCF Energy $\left(P C M / m P W 1 P W 91 / 6-31+G^{* *}\right)=-689.024574155$

03 c05

B3LYP/6-31G* Geometry

O $-0.545513-0.743061-0.306262$

C $1.141573 \quad 1.004424-0.671061$

C $-1.2370581 .584950-0.295778$

C $0.0368551 .974714-0.367236$

C $-1.6545520 .144232-0.432030$

C $0.573360-0.352605-1.097997$

C $1.610797-1.433422-0.994366$

C $2.699117-1.265620-0.237216$

C $2.897353-0.0703020 .620533$

$\begin{array}{lllll}\text { O } & 1.999266 & 0.943158 & 0.497635\end{array}$

H $0.253367-0.249429-2.149683$

H $1.7611891 .382974-1.497114$

C $-2.686745-0.2560020 .647078$

C -3.073837-1.729563 0.569212

O $3.775524-0.0003081 .450524$

H -2.133778 $-0.008742-1.421448$

O -3.8262630 .5984390 .552763$

H -2.038203 $2.295029-0.111174$

H $0.3207793 .012330-0.211838$

H $1.432080-2.361312-1.532219$

H $3.464164-2.028286-0.135021$

H $-2.246561-0.0394031 .626011$

H -2.199478 -2.3717780 .701227$

H -3.517969 - $1.962927-0.409189$

H $-3.809665-1.961907 \quad 1.345360$

H -4.351216 $0.302783-0.209039$

SCF Energy $\left(\mathrm{PCM} / \mathrm{mPW} 1 \mathrm{PW} 91 / 6-31+\mathrm{G}^{\star *}\right)=-689.025225798$

$03 \_06$

B3LYP/6-31G* Geometry

O $-0.682820-0.622884-0.483590$

C $1.1028231 .050204-0.659984$

C $-1.2538551 .729070-0.323143$

C $0.0393882 .057543-0.327361$

C $-1.7422480 .325633-0.583142$

C $0.477844-0.236893-1.207964$

C $1.453394-1.377609-1.152958$

C $2.518138-1.327375-0.346515$

C $2.742743-0.214248 \quad 0.608899$

$\begin{array}{lllll}O & 1.904670 & 0.855320 & 0.532333\end{array}$

H $\quad 0.203769-0.037271-2.258874$

H $1.7746221 .454505-1.431227$

C $-2.853336-0.092761 \quad 0.393441$

C $-2.345678-0.336569 \quad 1.813739$

O $3.586837-0.2548921 .475444$

H $-2.1824590 .271628-1.596234$

O $-3.455985-1.255674-0.167956$

H $-2.0132372 .485828-0.133559$

H $\quad 0.3673213 .069330-0.103507$

H $1.245535-2.251496-1.765237$

H $\quad 3.237159-2.136862-0.275238$

H $-3.5835310 .735878 \quad 0.404599$

H -1.8665890 .5593782 .222672$

H $-1.616979-1.1504551 .817333$

H -3.180054 -0.6015492 .475567$

H $-4.042443-1.6358630 .503781$

SCF Energy $\left(\mathrm{PCM} / \mathrm{mPW} 1 \mathrm{PW} 91 / 6-31+\mathrm{G}^{* *}\right)=-689.023845082$

04_c01

B3LYP/6-31G* Geometry

O $0.709296-0.627585-0.480732$

C $-1.0881741 .028024-0.699695$

C $1.2657851 .732891-0.397188$

C $-0.0291262 .052417-0.410927$

C $1.7692300 .328011-0.606963$

C $-0.463047-0.272339-1.210750$

C $-1.431606-1.416224-1.114183$

C $-2.491066-1.345522-0.302445$

C -2.712266 $-0.204180 \quad 0.621276$ $\begin{array}{llll}0 & -1.872912 & 0.861186 & 0.509502\end{array}$

H - $0.190633-0.107032-2.266992$

H -1.774294 $1.399098-1.474644$

C $2.824183-0.073200 \quad 0.443926$

C $3.408171-1.4638900 .182444$

O $-3.550955-0.2221741 .493141$

H $2.2210550 .238975-1.612678$

$\begin{array}{llll}\text { O } & 2.276753 & 0.032652 & 1.749516\end{array}$

H $2.0206792 .495443-0.216926$

H $-0.3636973 .066891-0.211587$

H - $1.227314-2.306315-1.704138$

H -3.208104-2.153899-0.203926

H 3.6297180 .6708830 .400276

H $3.919270-1.506485-0.787289$

H $2.618365-2.2215880 .187067$

H $4.130254-1.7078370 .967021$

H $1.414731-0.4166871 .719419$

SCF Energy $\left(P C M / m P W 1 P W 91 / 6-31+G^{* *}\right)=-689.026612250$

04 c02

B3LYP/6-31G* Geometry

O $0.539876-0.732652-0.317210$

C $-1.1313721 .017994-0.678090$

C $1.2570351 .584108-0.329068$

C $-0.0151361 .982386-0.396687$

C $1.6696920 .141516-0.446043$

C $-0.577450-0.342504-1.110419$

C $-1.623034-1.414768-0.998413$

C $-2.697916-1.239901-0.223734$

C $-2.872001-0.045736 \quad 0.641139$

O $-1.9687130 .962666 \quad 0.504189$

H $-0.258901-0.244105-2.162161$

H $-1.760093 \quad 1.400860-1.495141$

C $2.652457-0.3275610 .647600$

C $4.0115190 .356526 \quad 0.575765$

O

H $2.150971-0.038265-1.425348$

O $2.879271-1.721425 \quad 0.496848$

H $2.0501372 .313905-0.185408$

H $-0.2864093 .026791-0.266999$

H $-1.462528-2.340803-1.545117$

H -3.469156-1.995155-0.113556

H $2.179985-0.117990 \quad 1.621406$

H 3.9428901 .4253280 .801373

H $4.4596110 .228854-0.416093$

H $4.679936-0.1040371 .309037$

H $1.999802-2.1283370 .421076$

SCF Energy $\left(\mathrm{PCM} / \mathrm{mPW} 1 \mathrm{PW} 91 / 6-31+\mathrm{G}^{* *}\right)=-689.027578597$

04_c03

B3LYP/6-31G* Geometry

O $0.501650-1.022626-0.218426$

C $-1.0166880 .754270-0.970951$

C $1.4237601 .143148-0.836488$

C $0.1945521 .641895-0.979726$

C $1.685402-0.319101-0.591279$

C $-0.611646-0.720634-1.053532$

C $-1.746652-1.616924-0.647536$

C $-2.761500-1.143580 \quad 0.081914$

$\begin{array}{llll}C & -2.778209 & 0.239681 & 0.620703\end{array}$

$\begin{array}{llll}O & -1.793916 & 1.083126 & 0.209873\end{array}$

H - $-0.342075-0.920365-2.105288$

H - $1.6533790 .975954-1.839743$

C $2.706277-0.5688850 .534697$

C 2.3027110 .0490421 .870331

$\begin{array}{lllll}\text { O } & -3.584677 & 0.610487 & 1.443826\end{array}$

H $2.109900-0.767433-1.508267$

$\begin{array}{lllll}\text { O } & 3.938019 & -0.040673 & 0.040163\end{array}$

H $2.3032531 .779062-0.892781$

H $0.0275052 .706613-1.121104$

H - $1.698634-2.662518-0.941682$

H -3.593269 -1.763607 0.399896

H $2.778342-1.6619010 .649013$

H $3.028834-0.2152432 .649781$

H $1.321640-0.3218152 .180661$

H $2.254788 \quad 1.1403911 .798013$

H $4.586445-0.0851340 .759214$ 
SCF Energy $\left(P C M / m P W 1 P W 91 / 6-31+G^{* *}\right)=-689.025148621$

04_c04

B3LYP/6-31G* Geometry

O $0.499505-0.968760-0.416018$

C $-1.0148560 .917636-0.830997$

C $1.4275511 .265412-0.648519$

C $0.2010841 .789177-0.696260$

C $1.688574-0.216145-0.652533$

C $-0.617109-0.520121-1.177739$

C $-1.753276-1.470038-0.926180$

C $-2.757034-1.131427-0.111152$

C -2.7613540 .1340800 .664436$

$\begin{array}{lllll}\text { O } & -1.777716 & 1.035371 & 0.397232\end{array}$

H $-0.355492-0.530935-2.249968$

H -1.657504 $1.292733-1.640717$

C $2.709441-0.6628380 .423276$

C $2.262021-0.3600531 .849045$

O $-3.556034 \quad 0.356366 \quad 1.549822$

H $2.119924-0.500205-1.629645$

O $3.984911-0.0986690 .118747$

H $2.304417 \quad 1.908791-0.620196$

H $\quad 0.0407072 .863558-0.659242$

H $-1.713980-2.446847-1.401839$

H -3.587732 -1.7960180 .102351$

H 2.826489-1.745869 0.303557

H $1.285596-0.8093582 .048247$

H 2.1673520 .7216502 .012568

H $2.989342-0.7580262 .564112$

H 4.0653010 .7319620 .612233

SCF Energy $\left(\mathrm{PCM} / \mathrm{mPW} 1 \mathrm{PW} 91 / 6-31+\mathrm{G}^{\star *}\right)=-689.024295528$

04 c05

B3LYP/6-31G* Geometry

O $-0.505827 \quad 1.033476-0.135668$

C $1.019249-0.680678-1.017408$

C -1.418843-1.090425 -0.892876

C $-0.188017-1.572165-1.073852$

C -1.685429 $0.350595-0.551956$

C $0.6090590 .795190-0.990778$

C $1.7409551 .664787-0.523735$

C 2.7646321 .1442650 .159732

C $2.797109-0.2765950 .588401$

O $1.812136-1.0921920 .125705$

H $\quad 0.336252 \quad 1.069417-2.024981$

H $1.645076-0.836917-1.908159$

$\begin{array}{llll}\text { C } & -2.724058 & 0.517324 & 0.583169\end{array}$

C $-2.335533-0.1966401 .868597$

O $3.616760-0.7049841 .369129$

H -2.098867 $0.859747-1.447185$

O $-3.981254-0.0099390 .158817$

H -2.295433 -1.727752 - 0.976525

H $-0.015323-2.625843-1.277175$

H $1.6835002 .729726-0.735198$

H 3.5941451 .7444990 .518876

H -2.8031441 .5974840 .779685$

H -1.3470560 .1338452 .198697$

H -2.308387 -1.2803501 .717260$

H -3.0678470 .0224422 .651383$

H -4.3321820.568856-0.536295

SCF Energy $\left(P C M / m P W 1 P W 91 / 6-31+G^{* *}\right)=-689.025095981$

04_c06

B3LYP/6-31G* Geometry

O -0.9274520 .0601631 .423395$

$\begin{array}{llll}\text { C } & 1.227912 & 0.995880 & 0.772848\end{array}$

C $-0.823907 \quad 1.588566-0.495035$

C $0.4870141 .738384-0.306014$

C -1.6663990 .6760120 .345245$

C $0.449971-0.257147 \quad 1.208903$

C $0.672521-1.3878910 .237309$

C $1.758227-1.428044-0.541479$

C $2.813287-0.391102-0.464685$

$\begin{array}{lllll}\text { O } & 2.574297 & 0.687623 & 0.341461\end{array}$

H $\quad 0.795037-0.5791772 .200004$

H 1.3815821 .6485701 .638447

C $-2.409181-0.420732-0.459880$
C $-3.4993370 .142319-1.364311$

O $3.880826-0.486946-1.023663$

H $-2.434944 \quad 1.2624510 .867967$

O $-3.028122-1.3205070 .448750$

H $-1.3376482 .146354-1.274148$

H $1.0777632 .420115-0.912926$

H $-0.062405-2.1901440 .232257$

H $1.963454-2.244589-1.225456$

H $-1.669243-0.953239-1.080315$

H -3.081500 $0.741669-2.179766$

H $-4.2003050 .758136-0.789376$

H - $4.062564-0.685969-1.804493$

$\mathrm{H}-2.426263-1.3843241 .210864$

SCF Energy $\left(\mathrm{PCM} / \mathrm{mPW} 1 \mathrm{PW} 91 / 6-31+\mathrm{G}^{* *}\right)=-689.022138872$

05_c01

B3LYP/6-31G* Geometry

O $-0.739964-1.027422-0.281038$

C 0.8886890 .6198220 .339194

C $-1.3299511 .354068-0.390527$

C -0.1230041 .6916500 .070328$

C $-1.741929-0.070430-0.681562$

C $0.582674-0.586297-0.543944$

C $1.597139-1.651887-0.255584$

C $2.836497-1.2690790 .079181$

C 3.2319490 .1675320 .117677

$\begin{array}{lllll}\text { O } & 2.224930 & 1.097480 & 0.079073\end{array}$

H $0.665135-0.276843-1.600708$

H $\quad 0.8464880 .299948 \quad 1.390967$

C -3.019989 -0.5331510 .050681$

C $-4.2575720 .288058-0.283659$

$\begin{array}{lllll}O & 4.382627 & 0.535492 & 0.135731\end{array}$

H -1.913244 - $0.176072-1.767986$

O $-2.831695-0.4836151 .455776$

H -2.072503 $2.119903-0.598746$

H 0.1538202 .7222710 .275297

H $1.312290-2.695846-0.362480$

H $3.643325-1.9704750 .261752$

H -3.183294 -1.574762 -0.274365

H $-4.4422260 .304348-1.363676$

H $-4.155437 \quad 1.3163480 .076619$

H $-5.129671-0.1519410 .208640$

H - $1.998880-0.9540721 .627636$

SCF Energy $\left(\mathrm{PCM} / \mathrm{mPW} 1 \mathrm{PW} 91 / 6-31+\mathrm{G}^{\star *}\right)=-689.024410009$

05_c02

B3LYP/6-31G* Geometry

O $-0.898918-0.786993-0.207508$

$\begin{array}{llll}\text { C } & 0.909786 & 0.708940 & 0.270138\end{array}$

C $-1.1941991 .624023-0.583445$

C $0.0395401 .862374-0.129622$

C $-1.765577 \quad 0.235411-0.734033$

C $0.468185-0.540237-0.489766$

C $1.345756-1.680544-0.069370$

C $2.618631-1.4140180 .251805$

C $3.183539-0.038606 \quad 0.150724$

$\begin{array}{llll}\text { O } & 2.295844 & 0.994903 & -0.008632\end{array}$

H $\quad 0.596610-0.357105-1.571328$

H $\quad 0.8178970 .507512 \quad 1.347368$

C $-3.1269630 .030685-0.036517$

C $-3.0897690 .296648 \quad 1.469943$

$\begin{array}{llll}O & 4.369620 & 0.190772 & 0.158455\end{array}$

H -1.925463 $0.029264-1.806669$

O $-3.604315-1.277075-0.326732$

H -1.842770 $2.442404-0.890436$

H $0.4394492 .867614-0.027239$

H $\quad 0.940103-2.689445-0.074073$

H $3.333380-2.1828770 .524059$

H -3.842925 $0.712851-0.510895$

H -2.8184801 .3356791 .687435$

H -2.357978 -0.3554861 .958331$

H $-4.0756950 .094641 \quad 1.898341$

H -2.895546-1.885958 -0.058695

SCF Energy $\left(\mathrm{PCM} / \mathrm{mPW} 1 \mathrm{PW} 91 / 6-31+\mathrm{G}^{* *}\right)=-689.023987343$

$05 \_$c03

B3LYP/6-31G* Geometry 
$\begin{array}{llll}\text { O } & -0.773379 & 0.867699 & 0.037700\end{array}$

C $1.007826-0.673945-0.396507$

C $-1.188838-1.546215 \quad 0.242347$

C $0.064089-1.809012-0.138698$

C $-1.712364-0.1406380 .435480$

C $\quad 0.5551690 .5417520 .407399$

C $1.5049641 .668500 \quad 0.132180$

C $2.7885821 .369506-0.107485$

C $3.292022-0.032368-0.056400$

O $2.357991-1.034905-0.034629$

H 0.5925340 .2852001 .481312

H $1.008483-0.401056-1.462016$

C $-2.9989430 .116211-0.369904$

C -3.523866 $1.539281-0.194935$

O $4.466635-0.309761 \quad 0.009509$

H $-1.961900-0.0004851 .502822$

O $-3.934761-0.850753 \quad 0.109735$

H -1.903766 -2.340048 0.435658

H $0.426926-2.822910-0.285784$

H 1.1364292 .6908110 .170981

H $3.5508312 .123102-0.272918$

H -2.767035 -0.065508-1.431268

H $-2.7887072 .272582-0.536877$

H -3.7535101 .7326570 .859002$

H $-4.4433471 .680075-0.777327$

H $-4.760671-0.731089-0.382972$

SCF Energy $\left(\mathrm{PCM} / \mathrm{mPW} 1 \mathrm{PW} 91 / 6-31+\mathrm{G}^{* *}\right)=-689.022653088$

05_c04

B3LYP/6-31G* Geometry

$\begin{array}{lllll}O & -0.772691 & 0.866627 & 0.029721\end{array}$

C $1.013439-0.672569-0.401919$

C $-1.186864-1.547593 \quad 0.219697$

C $0.067397-1.808686-0.157869$

C $-1.710477-0.144160 \quad 0.422156$

C 0.5562330 .5411380 .401942

C 1.5066391 .6688040 .132240

C $2.7917551 .370265-0.099871$

C $3.295099-0.031549-0.044716$

O $2.360606-1.034228-0.030069$

H $\quad 0.589960 \quad 0.282482 \quad 1.475610$

H $1.022241-0.397220-1.466760$

C $-3.0129390 .105145-0.372631$

C -3.524704 $1.535471-0.227878$

O $4.468939-0.3090640 .030927$

H $-1.947815-0.0074931 .496177$

O $-3.997163-0.8513590 .018821$

H $-1.907076-2.342003 \quad 0.391214$

H $0.427119-2.821371-0.319975$

H 1.1372602 .6908940 .168278

H $3.5546562 .124027-0.261441$

H -2.808458 -0.110504-1.426790

H $-2.7843712 .256373-0.583759$

H -3.737739 1.7662740 .825634

H $-4.4489531 .658313-0.801072$

H -4.347824 -0.5731770 .880786$

SCF Energy $\left(P C M / m P W 1 P W 91 / 6-31+G^{* *}\right)=-689.022591675$

05_c05

B3LYP/6-31G* Geometry

$\begin{array}{llllll}O & -0.766177 & 0.886618 & 0.059294\end{array}$

C $0.998155-0.674684-0.368465$

C $-1.192838-1.5179370 .319836$

C $0.055442-1.799427-0.064487$

C -1.719726-0.108309 0.461019

$\begin{array}{llll}\text { C } & 0.563554 & 0.558343 & 0.420412\end{array}$

C 1.5168811 .6735170 .111498

C $2.7946561 .362639-0.143961$

C $3.291100-0.040708-0.079528$

O $2.350984-1.038082-0.026527$

H 0.6135930 .3210641 .498059

H $0.976208-0.422522-1.438772$

C $-2.9901300 .142415-0.388011$

C $-3.5796501 .522706-0.134863$

O $4.464240-0.325873-0.029521$

H -1.9930750 .0560921 .518669$

O $-3.995245-0.816089-0.055377$
H -1.886925 -2.3121460 .583341$

H $\quad 0.420860-2.818385-0.161772$

H $1.1554412 .698633 \quad 0.139612$

H $3.5583572 .108945-0.333799$

H -2.700308 $0.051824-1.445419$

H -2.839742 $2.301413-0.336622$

H -3.9045451 .6059690 .908657$

H $-4.4514391 .678723-0.777147$

H $-3.803509-1.633522-0.538451$

SCF Energy $\left(\mathrm{PCM} / \mathrm{mPW} 1 \mathrm{PW} 91 / 6-31+\mathrm{G}^{\star \star}\right)=-689.021996345$

05_c06

B3LYP/6-31G* Geometry

O $-0.882391-0.781304-0.179489$

C $0.938958 \quad 0.700886 \quad 0.291833$

C $-1.173576 \quad 1.631624-0.520189$

C $0.0663451 .861804-0.079643$

C $-1.747938 \quad 0.240923-0.686192$

C $0.477925-0.532832-0.480205$

C $1.356415-1.683720-0.091786$

C $2.635859-1.4285220 .213146$

C $3.206356-0.0550020 .125424$

O $2.3224140 .986541-0.001088$

H $\quad 0.594621-0.333481-1.560847$

H $\quad 0.8605490 .483798 \quad 1.367105$

C $-3.1183300 .089659-0.005598$

C $-3.037260 \quad 0.070632 \quad 1.520288$

$\begin{array}{lllll}\text { O } & 4.393935 & 0.169065 & 0.118411\end{array}$

H -1.915947 $0.056453-1.762246$

O $-3.686893-1.108861-0.525376$

H -1.822246 $2.456224-0.809418$

H 0.4697652 .8652560 .027842

H $0.942225-2.688941-0.104122$

H $3.351017-2.2049080 .462169$

H $-3.7201470 .958371-0.325773$

H -2.582197 0.9907921 .902835

H -2.441157 -0.782071 1.854360

H $-4.041909-0.0087681 .954404$

H -4.499616-1.288959-0.028432

SCF Energy $\left(\mathrm{PCM} / \mathrm{mPW} 1 \mathrm{PW} 91 / 6-31+\mathrm{G}^{\star \star}\right)=-689.021270649$

05_c07

B3LYP/6-31G* Geometry

O $-0.715358-1.068777-0.213954$

C 0.8996340 .6255500 .299759

C $-1.319311 \quad 1.305000-0.462623$

C $-0.1086371 .677672-0.043566$

C $-1.727470-0.141941-0.630362$

C $0.597261-0.631943-0.511518$

C $1.617965-1.673565-0.162822$

C $2.855971-1.2691970 .150271$

C $3.2464180 .168027 \quad 0.110416$

$\begin{array}{lllll}\text { O } & 2.237938 & 1.090483 & 0.020157\end{array}$

H $\quad 0.680397-0.383534-1.585586$

H $\quad 0.844306 \quad 0.368707 \quad 1.367096$

C -2.995327 $-0.514608 \quad 0.156005$

C $-4.2696170 .078173-0.451243$

$\begin{array}{lllll}\text { O } & 4.396822 & 0.540060 & 0.110673\end{array}$

H - $1.943787-0.320909-1.699964$

O $-2.803972-0.0760831 .497343$

H -2.069489 $2.048439-0.719820$

H 0.1716762 .7207560 .075349

H $1.335344-2.722626-0.207076$

H $3.664535-1.9567740 .373451$

H -3.055076-1.613804 0.117518

H $-4.413933-0.250661-1.487034$

H $-4.2467381 .172616-0.428012$

H $-5.145347-0.2469990 .123033$

H -3.526650 -0.439692 2.031389

SCF Energy $\left(\mathrm{PCM} / \mathrm{mPW} 1 \mathrm{PW} 91 / 6-31+\mathrm{G}^{* *}\right)=-689.020741687$

06_c01

B3LYP/6-31G* Geometry

O $0.766853-0.765286-0.813857$

C $-1.0491610 .773794-0.507230$

C $1.2449861 .614798-0.376357$

C $-0.062144 \quad 1.886654-0.331784$ 
C $1.7984280 .228317-0.592355$

C $-0.412304-0.535716-0.052628$

C $-1.412841-1.636504-0.241146$

C $-2.710827-1.346077-0.083045$

C $-3.167720 \quad 0.013766 \quad 0.318928$

$\begin{array}{lllll}\text { O } & -2.248815 & 1.030324 & 0.249746\end{array}$

H $-0.165720-0.446707 \quad 1.018840$

H -1.330078 $0.671699-1.565838$

C $2.725810-0.281700 \quad 0.536545$

C 3.9748410 .5700320 .726495

$\begin{array}{lllll}\text { O } & -4.277995 & 0.242618 & 0.736221\end{array}$

H $2.3873950 .209786-1.519131$

O $3.154433-1.596032 \quad 0.212472$

H $1.9771582 .411742-0.275262$

H $-0.4423582 .895912-0.199136$

H $-1.059746-2.640565-0.464143$

H $-3.496779-2.089484-0.158710$

H $2.147821-0.289157 \quad 1.477401$

H 3.7314461 .5623621 .118811

H $4.5162540 .681914-0.219982$

H 4.6415110 .0741591 .438091

H $2.362876-2.058847-0.112148$

SCF Energy $\left(\mathrm{PCM} / \mathrm{mPW} 1 \mathrm{PW} 91 / 6-31+\mathrm{G}^{\star \star}\right)=-689.023786443$

06_c02

B3LYP/6-31G* Geometry

O $0.919271-0.590857-0.992038$

C $-0.9757360 .803272-0.518756$

C $1.2650391 .777582-0.419499$

C $-0.0529361 .963028-0.296953$

C $1.8885410 .433585-0.727647$

C $-0.249102-0.495957-0.185126$

C $-1.191320-1.631803-0.445674$

C $-2.499111-1.433247-0.231388$

C $-3.020920-0.1415730 .294038$

$\begin{array}{llll}\text { O } & -2.164775 & 0.932186 & 0.285160\end{array}$

H $0.033325-0.4912030 .877207$

H $-1.2819590 .765113-1.574901$

C 2.8941860 .0177620 .379015

C $3.525902-1.3406290 .111255$

O $-4.129467-0.011404 \quad 0.756833$

H $2.4613090 .516639-1.662223$

$\begin{array}{llll}\text { O } & 2.277261 & -0.055848 & 1.664025\end{array}$

H $1.9580702 .608681-0.296612$

H $-0.4885182 .935993-0.084181$

H $-0.786707-2.591267-0.758524$

H $-3.243181-2.213645-0.347365$

H 3.6864840 .7879360 .384837

H $4.071404-1.333334-0.838651$

H $2.753675-2.1125570 .062431$

H $4.224692-1.5896590 .914995$

H 1.8858330 .8125641 .855556

SCF Energy $\left(P C M / m P W 1 P W 91 / 6-31+G^{\star *}\right)=-689.021607578$

06_c03

B3LYP/6-31G* Geometry

O $0.912788-0.570763-0.936708$

C -1.001339 $0.807429-0.507192$

C $1.2246641 .797882-0.330507$

C $-0.0957671 .973719-0.253594$

C $1.8641740 .474591-0.687138$

C $-0.265624-0.478364-0.142435$

C $-1.185464-1.632381-0.401986$

C $-2.500326-1.450886-0.219682$

C $-3.053255-0.157813 \quad 0.270033$

$\begin{array}{llll}O & -2.216136 & 0.927107 & 0.262070\end{array}$

H $0.004270-0.4472060 .922570$

H $-1.2799310 .756218-1.570439$

C 2.9295850 .0423850 .342553

C $3.605282-1.272596-0.041744$

O $-4.175858-0.0399040 .703503$

H $2.3954290 .593260-1.642838$

O $2.283375-0.048241 \quad 1.611781$

H $1.9100872 .622676-0.147593$

H $-0.5438702 .935821-0.020645$

H $-0.760868-2.590961-0.690883$

H -3.229977 -2.244479-0.338397
H 3.6882620 .8451850 .363389

H $4.120271-1.185317-1.005317$

H $2.862250-2.070949-0.115282$

H $4.355094-1.5535440 .708715$

H $2.914057-0.4300842 .241718$

SCF Energy $\left(\mathrm{PCM} / \mathrm{mPW} 1 \mathrm{PW} 91 / 6-31+\mathrm{G}^{* *}\right)=-689.020913440$

06_c04

B3LYP/6-31G* Geometry

O $0.918105-0.575271-0.807014$

C - $-1.0236770 .817065-0.472926$

C $1.1859091 .811756-0.208766$

C $-0.136758 \quad 1.982025-0.161003$

C $1.8450230 .514134-0.609487$

C $-0.298151-0.466024-0.076332$

C $-1.199676-1.627437-0.371603$

C $-2.522230-1.454515-0.251069$

C $-3.104844-0.163824 \quad 0.209721$

$\begin{array}{llll}\text { O } & -2.272435 & 0.924637 & 0.240101\end{array}$

H $-0.089859-0.4153141 .003517$

H -1.249748 $0.770244-1.548598$

C $2.965610 \quad 0.0690230 .363334$

C $3.849138-1.022171-0.241544$

O $-4.246564-0.0513170 .589603$

H $2.3098110 .664367-1.595674$

O $2.423185-0.3410421 .616247$

H 1.8620022 .6348200 .012842

H -0.5971382 .9319530 .095716$

H $-0.756451-2.582793-0.642795$

H $-3.240325-2.252538-0.404972$

H 3.5805970 .9483020 .592366

H $4.364567-0.666454-1.140979$

H $3.242693-1.890872-0.525196$

H $4.601586-1.3400580 .486451$

H $2.031873-1.2179081 .473874$

SCF Energy $\left(\mathrm{PCM} / \mathrm{mPW} 1 \mathrm{PW} 91 / 6-31+\mathrm{G}^{* *}\right)=-689.019879330$

06_c05

B3LYP/6-31G* Geometry

O $0.725566-0.885306-0.892238$

C $-0.9714410 .779879-0.460047$

C $1.3728641 .430457-0.285385$

C $0.0927201 .790283-0.162939$

C $1.801426 \quad 0.060855-0.750997$

C $-0.435469-0.611062-0.126024$

C $-1.513555-1.612210-0.420593$

C $-2.789194-1.244632-0.243172$

C $-3.1501620 .101353 \quad 0.281978$

$\begin{array}{llll}\text { O } & -2.159938 & 1.049008 & 0.311212\end{array}$

H $-0.205730-0.6324180 .952106$

H $-1.2461380 .799107-1.525161$

C $2.937893-0.5558650 .097850$

C $2.604017-0.7560301 .574391$

$\begin{array}{lllll}\text { O } & -4.245764 & 0.371873 & 0.714750\end{array}$

H $2.2197280 .167687-1.761375$

$\begin{array}{llll}\text { O } & 4.041618 & 0.334719 & -0.078361\end{array}$

H $2.1833462 .126383-0.089698$

H $-0.2096722 .790754 \quad 0.134240$

H - $-1.228487-2.612673-0.736917$

H -3.626094 -1.917876-0.393500

H $3.161880-1.534389-0.354884$

H $1.780279-1.4667111 .698006$

H 2.3307530 .1913482 .050192

H $3.470602-1.1699092 .106206$

H $4.783071-0.0018140 .447517$

SCF Energy $\left(P C M / m P W 1 P W 91 / 6-31+G^{\star \star}\right)=-689.019269464$

06 c06

B3LYP/6-31G* Geometry

O $0.722464-0.900830-0.869725$

C $-0.9727020 .771634-0.464088$

C $1.3714761 .420061-0.283506$

C $0.0915651 .782920-0.168513$

C $1.7986520 .046595-0.736554$

C $-0.441152-0.616390-0.109844$

C $-1.519170-1.619637-0.396485$

C $-2.795351-1.247328-0.233357$ 
C -3.1584120 .1067660 .268765$

$\begin{array}{lllll}\text { O } & -2.166306 & 1.053250 & 0.293307\end{array}$

H $-0.213239-0.6254230 .968608$

H -1.237895 $0.778515-1.531855$

C $2.948078-0.5551450 .116850$

C $2.629918-0.710671 \quad 1.596793$

$\begin{array}{lllll}\text { O } & -4.257160 & 0.386000 & 0.687295\end{array}$

H $2.2015860 .143268-1.758122$

$\begin{array}{lllllll}\text { O } & 4.095435 & 0.291844 & 0.025106\end{array}$

H $2.1831182 .112143-0.078042$

H -0.2105432 .7832650 .129260$

H -1.234072 -2.625607-0.694750

H $-3.632164-1.921714-0.378933$

H $3.166709-1.546951-0.307594$

H $1.792633-1.4001301 .741597$

H 2.3770160 .2538762 .048585

H $3.503133-1.1152422 .117424$

H $4.4308360 .249061-0.884407$

SCF Energy $\left(\mathrm{PCM} / \mathrm{mPW} 1 \mathrm{PW} 91 / 6-31+\mathrm{G}^{\star *}\right)=-689.019320331$

07_c01

B3LYP/6-31G* Geometry

O -0.7643780 .7734620 .780013$

C $1.063235-0.7612390 .502273$

C $-1.224099-1.6116200 .341106$

C $0.084215-1.8777600 .307256$

C $-1.778523-0.2268020 .582859$

$\begin{array}{llll}\text { C } & 0.422288 & 0.543205 & 0.036981\end{array}$

C $1.412495 \quad 1.651773 \quad 0.235405$

$\begin{array}{llll}\text { C } 2.714588 & 1.371287 & 0.093828\end{array}$

C $3.1862640 .014192-0.300371$

O $2.276839-1.009102-0.238122$

H $0.1930690 .448255-1.038431$

H $1.330614-0.6549141 .563951$

C -2.757429 $0.219960-0.528662$

C $-3.3790161 .583057-0.236121$

O $4.303643-0.205200-0.705773$

H $-2.349771-0.237317 \quad 1.521324$

O $-3.748797-0.806829-0.587705$

H -1.965826 -2.3910660 .199694$

H $\quad 0.470653-2.8819940 .155115$

H 1.0468862 .6522540 .453984

H 3.4942692 .1204280 .178330

H -2.201480 $0.264559-1.479813$

H -2.607761 2.352481 -0.142204

H -3.9532081 .5455910 .696642$

H $-4.058857 \quad 1.878030-1.045665$

H $-4.401151-0.551270-1.257547$

SCF Energy $\left(\mathrm{PCM} / \mathrm{mPW} 1 \mathrm{PW} 91 / 6-31+\mathrm{G}^{\star *}\right)=-689.020835692$

07 c02

B3LYP/6-31G* Geometry

O $-0.726890-0.897124-1.022176$

C $0.9441300 .753923-0.541258$

C $-1.4143651 .408462-0.505852$

C $-0.1368681 .776705-0.367446$

C $-1.838247-0.016121-0.793600$

C $0.397108-0.626645-0.193591$

C $1.494050-1.624680-0.408485$

C $2.756157-1.241087-0.171641$

C 3.0801790 .1208100 .335449

$\begin{array}{lllll}\text { O } & 2.083096 & 1.063863 & 0.285126\end{array}$

H $0.091616-0.6399620 .862156$

H $1.2787460 .738551-1.589353$

C $-2.775817-0.596778 \quad 0.298492$

C -4.1618160 .0539230 .310929$

$\begin{array}{lllll}\text { O } & 4.148680 & 0.414081 & 0.817743\end{array}$

H $-2.395650-0.034652-1.740376$

O $-2.174838-0.5647621 .592851$

H $-2.2109232 .145115-0.424543$

H $0.1541352 .806677-0.176857$

H $1.234363-2.637363-0.707166$

H $3.604242-1.911999-0.254003$

H $-2.879005-1.6628920 .071627$

H $-4.658758-0.036578-0.662076$

H $-4.106401 \quad 1.1201090 .564502$

H $-4.786603-0.4350071 .064134$
H -2.033208 $0.368467 \quad 1.825634$

SCF Energy $\left(\mathrm{PCM} / \mathrm{mPW} 1 \mathrm{PW} 91 / 6-31+\mathrm{G}^{* *}\right)=-689.021033796$

07_c03

B3LYP/6-31G* Geometry

O $-0.724647-0.844324-1.014185$

C $0.9643400 .777481-0.507701$

C $-1.385506 \quad 1.444304-0.385693$

C $-0.1041821 .800297-0.271663$

C $-1.8241880 .047970-0.769209$

C $0.405913-0.604666-0.184354$

C $1.486533-1.613004-0.431242$

C $2.756673-1.251630-0.204093$

C 3.1065910 .0968550 .322456

$\begin{array}{llll}\text { O } & 2.125101 & 1.053317 & 0.303188\end{array}$

H $0.105781-0.6313860 .872855$

H $1.2804240 .784462-1.561629$

C $-2.817376-0.5919810 .223668$

$\begin{array}{llll}\text { C } & -4.185502 & 0.094356 & 0.239111\end{array}$

$\begin{array}{lllll}\mathrm{O} & 4.187720 & 0.363386 & 0.793824\end{array}$

H $-2.3471170 .100042-1.734859$

O $-2.209671-0.5654461 .516032$

H -2.176636 $2.172026-0.224399$

H $0.1975552 .814561-0.024359$

H $1.210052-2.616035-0.747599$

H $3.594397-1.932190-0.310847$

H -2.939784 -1.632958 -0.113674

H $-4.6431460 .100546-0.757159$

H $-4.105901 \quad 1.1252550 .598385$

H $-4.868080-0.439707 \quad 0.911235$

H -2.755353 -1.099966 2.113507

SCF Energy $\left(\mathrm{PCM} / \mathrm{mPW} 1 \mathrm{PW} 91 / 6-31+\mathrm{G}^{* *}\right)=-689.020473659$

07_c04

B3LYP/6-31G* Geometry

O $\begin{array}{lllll}-0.761963 & 0.774517 & 0.754143\end{array}$

C $1.065744-0.7633110 .490153$

C $-1.219671-1.610368 \quad 0.304267$

C $0.088453-1.8775250 .273874$

C - $1.774935-0.2298890 .563197$

C 0.4305730 .5429720 .020282

C $1.4194051 .650648 \quad 0.230535$

C $2.723110 \quad 1.369571 \quad 0.106440$

C $3.2003090 .012360-0.280201$

O $2.289062-1.010515-0.232704$

H $\quad 0.2105020 .450166-1.057089$

H $1.316634-0.6602221 .556202$

C $-2.7876360 .206635-0.531085$

C $-3.3650331 .594409-0.269072$

O $4.323758-0.208039-0.667324$

H $-2.324648-0.2459981 .517669$

O $-3.814861-0.778780-0.648404$

H - $1.961082-2.3846440 .133929$

H $\quad 0.475512-2.8777370 .099007$

H 1.0517672 .6516750 .443038

H 3.5016622 .1187040 .200795

H -2.278960 $0.195100-1.501857$

H -2.574500 $2.347783-0.220979$

H -3.901682 1.6153570 .690240

H $-4.0689591 .863412-1.062762$

H -4.416137 -0.6640690 .105781$

SCF Energy $\left(\mathrm{PCM} / \mathrm{mPW} 1 \mathrm{PW} 91 / 6-31+\mathrm{G}^{* *}\right)=-689.020451645$

07_c05

B3LYP/6-31G* Geometry

$\begin{array}{llll}\text { O } & 0.761088 & 0.799620 & -0.800794\end{array}$

C $-1.061525-0.742215-0.530844$

C $1.229786-1.588970-0.422572$

C $-0.077638-1.861744-0.384172$

C $1.784056-0.195323-0.608092$

C $-0.4165540 .554263-0.049349$

C $-1.4133621 .662589-0.212749$

C $-2.7123711 .374346-0.057860$

C $-3.173814 \quad 0.0088310 .318367$

O $-2.260460-1.0108440 .224068$

H $-0.1753530 .439648 \quad 1.021581$

H $-1.345387-0.615370-1.585928$ 
C 2.7371490 .2401190 .540875

C 3.4654421 .5329890 .203585

O $-4.283486-0.223790 \quad 0.736015$

H $2.371711-0.178867-1.535890$

$\begin{array}{lllll}\text { O } & 3.733288 & -0.758951 & 0.765585\end{array}$

H $1.966142-2.385447-0.354853$

H $-0.459626-2.873870-0.279466$

H - $1.0538342 .668130-0.417252$

H -3.495791 2.121956-0.117266

H 2.1328860 .3860681 .449965

H $2.7504122 .320968-0.047543$

H $4.1297241 .374401-0.653776$

H 4.0754221 .8565921 .052187

H $3.333238-1.471803 \quad 1.286123$

SCF Energy $\left(\mathrm{PCM} / \mathrm{mPW} 1 \mathrm{PW} 91 / 6-31+\mathrm{G}^{* *}\right)=-689.020346346$

07_c06

B3LYP/6-31G* Geometry

O $-0.907835-0.651432-0.792047$

C $1.0051720 .792289-0.500521$

C $-1.2253661 .765359-0.334821$

C $0.094161 \quad 1.955325-0.256581$

C $-1.8521530 .441533-0.686510$

C $0.300935-0.490158-0.062419$

C $1.229659-1.642512-0.308889$

C $2.546721-1.436774-0.180170$

C $3.095510-0.1195420 .245286$

$\begin{array}{lllll}O & 2.238246 & 0.951569 & 0.228124\end{array}$

H $0.095452-0.4114891 .017001$

H $1.2492020 .706166-1.569760$

C -3.0242660 .0195920 .230620$

C $-2.662386-0.1533021 .707624$

$\begin{array}{lllll}\text { O } & 4.227800 & 0.031634 & 0.638257\end{array}$

$\mathrm{H}-2.2822940 .515004-1.695023$

O $-3.610070-1.164452-0.295622$

$\mathrm{H}-1.9179102 .589971-0.178263$

H $0.5356592 .922966-0.035038$

H $0.808958-2.615627-0.550457$

H $3.283370-2.223664-0.299448$

H -3.7968940 .7939720 .139373$

H -2.2372300 .7617972 .134957$

H $-1.937530-0.964681 \quad 1.839165$

$\mathrm{H}-3.561357-0.4185712 .271839$

H $-2.872477-1.785249-0.425750$

SCF Energy $\left(P C M / m P W 1 P W 91 / 6-31+G^{* *}\right)=-689.021091745$

\section{$07 \quad$ c07}

B3LYP/6-31G* Geometry

O $-0.740337-0.820864-0.954573$

C $0.9688520 .808192-0.462122$

C $-1.371423 \quad 1.477357-0.288866$

C $-0.0882971 .824988-0.166121$

C $-1.8240140 .119102-0.768129$

C $0.408844-0.577289-0.153207$

C $1.478157-1.590737-0.434415$

C $2.755273-1.242637-0.230571$

C 3.1265600 .0954310 .307806

$\begin{array}{lllll}\text { O } & 2.149533 & 1.056618 & 0.326892\end{array}$

H $\quad 0.141223-0.6002300 .915035$

H $1.2567640 .837688-1.523571$

C -2.914491 -0.5693010 .090975$

$\begin{array}{llll}\text { C } & -4.125035 & 0.310687 & 0.375595\end{array}$

$\begin{array}{lllll}\text { O } & 4.219362 & 0.347980 & 0.757661\end{array}$

H $-2.2532530 .246895-1.773665$

O $-2.394991-0.999317 \quad 1.346628$

H -2.157459 2.201342 -0.092796

H 0.2206092 .8220570 .135321

H $1.187877-2.585712-0.763824$

H $3.584959-1.927166-0.369382$

H -3.239252 - $1.445972-0.491189$

H $-4.5313880 .740220-0.547271$

H -3.8590281 .1244351 .057389$

H $-4.908531-0.2836760 .854230$

H -1.797623 -1.739979 1.161205

SCF Energy $\left(\mathrm{PCM} / \mathrm{mPW} 1 \mathrm{PW} 91 / 6-31+\mathrm{G}^{* *}\right)=-689.019519037$

08_c01
B3LYP/6-31G* Geometry

O $0.910015-0.753917-0.200877$

C $-0.931425 \quad 0.706048 \quad 0.284325$

C $1.1701391 .668047-0.515705$

C $-0.0735291 .879452-0.079390$

C $1.7699320 .291895-0.691332$

C $-0.459034-0.520028-0.491958$

C $-1.320098-1.684531-0.104790$

C -2.601759-1.446999 0.203938

C $-3.189778-0.0800740 .118296$

O $-2.3189790 .971374-0.008960$

H $-0.576919-0.318301-1.571158$

H $-0.8513610 .487707 \quad 1.359507$

C 3.1047870 .1220090 .066193

C $3.802964-1.196537-0.278124$

$\begin{array}{lllll}\text { O } & -4.380073 & 0.127104 & 0.111004\end{array}$

H $1.9541270 .121009-1.767278$

$\begin{array}{lllll}\text { O } & 2.893447 & 0.255396 & 1.464222\end{array}$

H $1.8168422 .502509-0.778931$

H $-0.4848182 .877027 \quad 0.048429$

H $-0.894580-2.685078-0.122263$

H -3.305996 -2.2333330 .452749$

H $3.7549600 .959636-0.214056$

H $4.058997-1.246076-1.343377$

H $3.157977-2.049687-0.043002$

H $4.723862-1.2844860 .305591$

H $2.178434-0.3662841 .681958$

SCF Energy $\left(\mathrm{PCM} / \mathrm{mPW} 1 \mathrm{PW} 91 / 6-31+\mathrm{G}^{* *}\right)=-689.023988132$

08_c02

B3LYP/6-31G* Geometry

$\begin{array}{lllll}O & 0.770750 & 0.867909 & 0.081033\end{array}$

C $-0.990348-0.680173-0.384102$

C $1.204760-1.5418560 .278050$

C $-0.044299-1.810448-0.113333$

C $1.733092-0.1393420 .456816$

C $-\begin{array}{llll}0.559171 & 0.532381 & 0.437427\end{array}$

C $-1.515173 \quad 1.6535850 .160711$

C $-2.7920881 .345877-0.102237$

C $-3.284551-0.060504-0.075632$

O $-2.341513-1.056073-0.049190$

H $-0.6029020 .267121 \quad 1.508305$

H $-0.971261-0.398949-1.447073$

C $2.9804640 .179413-0.394969$

C $4.200157-0.650203-0.016133$

O $-4.456807-0.349352-0.032187$

H $2.0032630 .019101 \quad 1.515625$

O $3.3312621 .543509-0.213644$

H $1.896967-2.3490310 .504301$

H $-0.407145-2.826521-0.243051$

H -1.1587692 .6793010 .218876$

H -3.558143 $2.094978-0.270165$

H $2.712426-0.012331-1.447474$

H $4.055200-1.711132-0.242472$

H $4.429476-0.539848 \quad 1.049720$

H $5.063904-0.293671-0.584781$

H $2.5103872 .050651-0.329493$

SCF Energy $\left(\mathrm{PCM} / \mathrm{mPW} 1 \mathrm{PW} 91 / 6-31+\mathrm{G}^{* \star}\right)=-689.024958572$

08_c03

B3LYP/6-31G* Geometry

O $0.731738-1.050522-0.243479$

$\begin{array}{llll}\text { C } & -0.893411 & 0.615727 & 0.338797\end{array}$

C $1.3277391 .331285-0.398814$

C $0.121695 \quad 1.6817800 .055851$

C $1.720135-0.102851-0.668986$

C $-0.586401-0.609452-0.519380$

C $-1.605838-1.665013-0.212568$

C $-2.845813-1.272495 \quad 0.108474$

C -3.2373510 .1653920 .116071$

O $-2.228317 \quad 1.0913850 .063611$

H $-0.667965-0.319933-1.582594$

H -0.8577610 .3143091 .396069$

C $3.036752-0.5167280 .015242$

C $3.014994-0.3585141 .532926$

O

H $1.882312-0.216381-1.755147$ 
O $4.0408690 .290361-0.600179$

H $2.0925762 .071873-0.615284$

H -0.1525662 .7162860 .245430$

H - $-1.322361-2.711564-0.294698$

H -3.655147 -1.9679640 .302413$

H $3.193027-1.578290-0.234260$

H $2.201975-0.9492491 .965380$

H 2.8770690 .6905491 .814005

H $3.957926-0.7123051 .968950$

H $4.8821740 .104470-0.156271$

SCF Energy $\left(\mathrm{PCM} / \mathrm{mPW} 1 \mathrm{PW} 91 / 6-31+\mathrm{G}^{\star *}\right)=-689.022350122$

$08 \_04$

B3LYP/6-31G* Geometry

O $0.729538-1.055694-0.219781$

C -0.8992140 .6186850 .335303$

C $1.3268551 .323590-0.396478$

C 0.1191021 .6803750 .048184

C $1.718607-0.112775-0.649987$

C $-0.588745-0.618153-0.504498$

C $-1.609688-1.668987-0.186516$

C $-2.851146-1.2711290 .121908$

C $-3.2419890 .166898 \quad 0.104124$

$\begin{array}{lllll} & \text { O } & -2.231506 & 1.091480 & 0.042948\end{array}$

H $-0.666960-0.343048-1.571944$

H -0.8715640 .3320131 .396825$

C $3.046979-0.5135420 .035231$

C $3.039662-0.3094151 .542242$

$\begin{array}{lllll}0 & -4.391761 & 0.539040 & 0.097062\end{array}$

H $1.868286-0.238472-1.740835$

O $4.1146490 .268163-0.499204$

H $2.0978302 .060227-0.605697$

H -0.1516842 .7164400 .233965$

H $-1.325941-2.716750-0.249914$

H $-3.661618-1.9631240 .323258$

H $3.201398-1.581709-0.182648$

H $2.211084-0.8632761 .992279$

H 2.9299620 .7515501 .788332

H $3.981199-0.6661091 .970175$

H $4.258112-0.004930-1.419083$

SCF Energy $\left(\mathrm{PCM} / \mathrm{mPW} 1 \mathrm{PW} 91 / 6-31+\mathrm{G}^{* *}\right)=-689.022431620$

08 c05

B3LYP/6-31G* Geometry

O $0.731469-1.046546-0.283349$

C $-0.8854340 .612078 \quad 0.334790$

C $1.3273601 .334031-0.416949$

$\begin{array}{llll}\text { C } & 0.125144 & 1.682160 & 0.050278\end{array}$

C $1.727581-0.096859-0.689447$

C $-0.587547-0.600405-0.545113$

C $-1.606201-1.658867-0.245574$

C -2.842071 -1.2702100 .095487$

C -3.2319560 .1673890 .133044$

$\begin{array}{llll}\text { O } & -2.221761 & 1.093811 & 0.084816\end{array}$

H - $-0.676653-0.295201-1.603192$

H $-0.833451 \quad 0.2951191 .386753$

C $3.044715-0.5181540 .008100$

C $2.979690-0.4528431 .530346$

O -4.3808520 .5408430 .160088$

H $1.903442-0.201581-1.774061$

$\begin{array}{lllll}\text { O } & 4.125150 & 0.246697 & -0.523537\end{array}$

H $2.0719832 .089047-0.658929$

H -0.1538892 .7161850 .235279$

H - $1.325654-2.704284-0.348950$

H -3.650205 -1.9680190 .285927$

H $3.238533-1.551687-0.300094$

H $2.164519-1.0767561 .906973$

H $2.802146 \quad 0.574821 \quad 1.874229$

H $3.922301-0.8052841 .961090$

H $4.197196 \quad 1.0592760 .001060$

SCF Energy $\left(\mathrm{PCM} / \mathrm{mPW} 1 \mathrm{PW} 91 / 6-31+\mathrm{G}^{* *}\right)=-689.021643950$

Cartesian Coordinates of the most stable conformations found for compounds (R)-MTPA-3, (S)-MTPA-3, (R)-MTPA-4 and (S)MTPA-4 at the PCM/mPW1PW91/6-31+G**//B3LYP/6-31G* level of theory.

R-MPTA-3 001
B3LYP/6-31G* Geometry

O $-2.250003 \quad 0.107248-0.203630$

C $-4.582328-0.229471-0.878310$

C $-2.784118-1.702579-1.734460$

C $-4.087583-1.464167-1.577859$

C $-1.719468-0.795385-1.168677$

C $-3.4364440 .759152-0.645288$

C -3.8060881 .7840840 .388330$

C $-4.803597 \quad 1.5554401 .248005$

C $-5.524128 \quad 0.259426 \quad 1.306624$

$\begin{array}{llll}0 & -5.247654 & -0.655582 & 0.336822\end{array}$

H -3.240749 $1.259795-1.609238$

H $-5.3402150 .271975-1.497564$

C $-0.599641-1.599762-0.483691$

C $-1.027951-2.2969440 .797510$

O $-6.284299-0.0280882 .203124$

H -1.258530 -0.225064-1.993611

O $0.482735-0.698954-0.118083$

H -2.442928 -2.570242 -2.296278

H $-4.837575-2.147132-1.968040$

H $-3.216102 \quad 2.696017 \quad 0.431321$

H -5.0911772 .2682912 .013756$

H $-0.204525-2.310321-1.216232$

H -1.843715 -2.9959070 .588380$

H - $1.379426-1.5665191 .529268$

H - $0.187682-2.8538961 .221712$

C $1.349646-0.357081-1.082640$

O $1.312246-0.745927-2.224600$

C $2.4779420 .563911-0.519412$

$\begin{array}{llll}\text { C } & 1.842351 & 1.783732 & 0.203157\end{array}$

O $3.132796 \quad 1.022274-1.683015$

C $4.3474151 .764170-1.556107$

H $4.8317601 .689998-2.532545$

H $5.0095541 .342667-0.792472$

H $4.1542742 .816896-1.327844$

C $3.386901-0.3029850 .365030$

C $5.094359-2.0037381 .806987$

C $3.508435-0.171468 \quad 1.753047$

C $4.126702-1.298642-0.292444$

C $4.974002-2.1420060 .422507$

C $4.360441-1.0182702 .465739$

H 2.9449540 .5805742 .290244

H $4.033751-1.400084-1.369671$

H $5.539703-2.906964-0.102456$

H $4.447244-0.9017583 .542568$

H $5.755210-2.6596492 .367108$

F 1.0904021 .4680211 .273286

F 2.8020162 .6331810 .642532

F $1.0607512 .463138-0.657111$

SCF Energy $\left(\mathrm{PCM} / \mathrm{mPW} 1 \mathrm{PW} 91 / 6-31+\mathrm{G}^{\star *}\right)=-1524.12305621$

R-MPTA-3 002

B3LYP/6-31 G* Geometry

O 2.2436220 .1304990 .114566

C $4.553785-0.1411380 .895500$

C $2.695733-1.3908631 .954681$

C $4.008363-1.2183281 .789905$

C $1.669572-0.5695871 .212882$

C 3.4426330 .8182050 .457446

C $3.863228 \quad 1.626737-0.735984$

C $4.8754321 .224036-1.510276$

C $5.563156-0.075938-1.312513$

O $5.235779-0.794956-0.203222$

H 3.2446661 .4919121 .309122

H $\quad 5.310430 \quad 0.445697 \quad 1.436280$

C $0.535314-1.4528520 .664185$

C $0.945616-2.353878-0.489198$

O $6.339207-0.536668-2.118246$

H $1.214400 \quad 0.1523031 .913681$

O $-0.535703-0.601937 \quad 0.162281$

H $2.318013-2.1370892 .651281$

H $4.729857-1.8419622 .311128$

H $3.2989102 .527962-0.961663$

H $5.1999391 .779144-2.384243$

H $0.129779-2.0294201 .500707$

H $1.745660-3.029570-0.170705$

H $1.311244-1.758114-1.328077$ 
H $0.091789-2.952312-0.819320$ C -1.429059-0.145649 1.050037 O $-1.407034-0.3708962 .239972$ $\begin{array}{llll}\text { C }-2.566139 & 0.662824 & 0.353235\end{array}$ C $-1.9466061 .716715-0.606187$ O -3.3800691 .3021381 .309011$ C - $2.829728 \quad 2.296628 \quad 2.180349$ H -1.8854661 .9755532 .626215$ H -3.574366 2.4191382 .969735 H -2.6956283 .2517351 .661594$ C $-3.496262-0.351384-0.334208$ C $-5.273243-2.257713-1.380506$ C $-4.125907-1.2801930 .508439$ C $-3.771995-0.382190-1.705198$ C $-4.657643-1.331630-2.220515$ C $-5.004555-2.227736-0.010674$ H $-3.928465-1.2473831 .574922$ H $-3.3110110 .322983-2.383725$ H $-4.864437-1.340191-3.287195$ H -5.482078 -2.939869 0.656842 H $-5.959767-2.994768-1.788230$ F -1.354696 $1.193753-1.694358$ F -2.888855 2.579402 -1.027366 F - $0.9975682 .436001 \quad 0.044876$ SCF Energy $\left(\mathrm{PCM} / \mathrm{mPW} 1 \mathrm{PW} 91 / 6-31+\mathrm{G}^{* *}\right)=-1524.12215112$

R-MPTA-3_005

B3LYP/6-31 G* Geometry

O -2.441657 -1.196299-1.029839

C $-3.748231-0.4788210 .912614$

C $-2.093375-2.3111121 .098931$

C $-2.897216-1.432621 \quad 1.700030$

C $-1.935650-2.372243-0.400595$

C $-3.743691-0.847147-0.572767$

C $-4.2431750 .290981-1.416084$

C $-4.244881 \quad 1.539847-0.940197$

C $-3.6464631 .886480 \quad 0.371543$

$\begin{array}{llll}\text { O } & -3.261455 & 0.863806 & 1.178954\end{array}$

H -4.417245-1.714396-0.690999

H $-4.787607-0.5134191 .269031$

C $-0.482443-2.530835-0.864137$

C $0.072812-3.939921-0.680047$

O -3.4284213 .0308200 .707620$

H -2.487027 -3.250135 -0.789388

O $0.313167-1.595577-0.092593$

H -1.513019-3.017563 1.686773

H $-2.966015-1.3808672 .783294$

H -4.573476 $0.068585-2.427534$

H $-4.5888202 .388934-1.521353$

H -0.431111 -2.240257 -1.916266

H $-0.523297-4.662687-1.247939$

H $\quad 0.074640-4.2377740 .373698$

H $1.099962-3.980239-1.051368$

C $1.412549-1.107910-0.686518$

O $1.759503-1.366229-1.815783$

C $2.176926-0.118598 \quad 0.241282$

C $3.697054-0.4007320 .066325$

O $1.812877-0.4589951 .565238$

C 2.2630740 .3648662 .643927

H 3.2920210 .1242492 .929453

H 1.5940010 .1390083 .477627

H 2.1913711 .4301992 .403752

C $1.7485251 .307054-0.143162$

C $0.8125383 .879982-0.759791$

C $2.5579472 .202915-0.852557$

C 0.4590061 .7090530 .240619

C $-0.0039092 .989472-0.059227$

C $2.0883073 .481999-1.156768$

H $3.5512651 .918226-1.174100$

H -0.1809751 .0159040 .777671$

H $-1.000005 \quad 3.2864450 .255515$

H $2.7296704 .167466-1.704345$

H $\quad 0.4536174 .878561-0.994607$

F $4.146259-0.271721-1.193390$

F 4.4286090 .4460980 .830509

$\begin{array}{llll}\text { F } & 3.975323-1.654011 & 0.473077\end{array}$

SCF Energy $\left(\mathrm{PCM} / \mathrm{mPW} 1 \mathrm{PW} 91 / 6-31+\mathrm{G}^{* *}\right)=-1524.12184234$
R-MPTA-3_006 B3LYP/6-31 G* Geometry

O $-2.4197740 .245305 \quad 0.120221$

C $-4.560881 \quad 1.145105-0.671343$

C $-2.7749280 .516416-2.267741$

C $-4.0484620 .850621-2.052568$

C $-1.7781980 .347543-1.147148$

C $-3.404231 \quad 1.2521890 .327363$

C -3.8957191 .1287231 .741017$

C -5.0853280 .5793642 .004418$

C $-5.932074-0.0329650 .950392$

O $-5.5320760 .120852-0.341728$

H -2.945549 $2.245800 \quad 0.181847$

H $-5.0977502 .104877-0.668639$

C $-0.922829-0.916443-1.346651$

C $-1.676429-2.219501-1.133717$

O $-6.908043-0.702861 \quad 1.201150$

H $-1.0927501 .212594-1.145820$

O $0.177497-0.921220-0.393414$

H $-2.4057920 .365107-3.280411$

H $-4.7560850 .938176-2.872787$

H -3.2399891 .4723042 .537019$

H $-5.4673700 .466861 \quad 3.013715$

H $-0.487861-0.863192-2.349529$

H $-2.513501-2.288006-1.835610$

H -2.073173 -2.268896-0.117650

H $-1.008324-3.069523-1.298838$

C $1.258085-0.198710-0.710303$

O $1.3739150 .490472-1.697319$

C $2.389724-0.3830600 .353003$

C $2.945242-1.8289030 .166620$

O $1.743260-0.2715721 .604762$

C $2.510402-0.5556282 .778506$

H 2.680867-1.6311392.892861

H $1.902030-0.1993463 .612403$

H $3.471452-0.0299882 .777091$

C 3.4595270 .6970490 .144587

C $5.3001242 .788086-0.221352$

C $4.5475960 .541185-0.722348$

C 3.2950941 .9176310 .811019

C 4.2096712 .9548500 .632688

C $5.4622911 .579608-0.898778$

H $4.689844-0.382700-1.269741$

H 2.4424802 .0503631 .468787

H 4.0669593 .8937271 .161073

H $6.3034261 .440198-1.572366$

H $6.0152903 .594211-0.360902$

F $3.147103-2.109459-1.143493$

F $4.122693-2.0035910 .802441$

F $2.090817-2.744528 \quad 0.646845$

SCF Energy $\left(\mathrm{PCM} / \mathrm{mPW} 1 \mathrm{PW} 91 / 6-31+\mathrm{G}^{* *}\right)=-1524.12161352$

R-MPTA-3_008

B3LYP/6-31 G* Geometry

O -2.903665 $1.239593-0.140650$

C $-4.331756-0.564332-0.993928$

C $-1.871716-0.840314-0.859958$

C -3.076732 -1.388252 -1.026021

C -1.685747 $0.625004-0.552785$

C $-4.0009350 .930480-0.997419$

C $-5.1771251 .749933-0.550069$

C $-6.173866 \quad 1.190287 \quad 0.142144$

C $-6.133782-0.2217850 .598859$

O $-5.106229-0.9942620 .153797$

H $-3.7356041 .199020-2.034424$

H $-4.941724-0.774290-1.884563$

C -0.6938510 .8649320 .600071$

C -0.5056382 .3343590 .945307$

O $-6.932318-0.677037 \quad 1.385673$

H $-1.292294 \quad 1.142310-1.448109$

O 0.5647330 .3059540 .122180

H $-0.969148-1.439044-0.940573$

H $-3.190500-2.454080-1.205585$

H $-5.1750872 .811521-0.784583$

H $-7.035121 \quad 1.753104 \quad 0.486814$

H -1.0100800 .2974001 .478670$ 
H -1.460882 2.7633331 .257686

H -0.1366042 .8906750 .076623$

H 0.2121462 .4379151 .763391

C $1.421221-0.1734591 .037995$

O $1.250240-0.1447662 .233852$

C $2.718484-0.7251290 .369588$

C $2.343648-1.636302-0.832599$

O $3.485588-1.4536021 .298655$

C $2.946971-2.6300141 .915968$

H $1.930204-2.4730772 .283102$

H $3.606613-2.8269882 .763472$

H 2.973519-3.486119 1.233631

C $3.5945890 .483430-0.002665$

C $5.2445952 .709889-0.456583$

C $4.0160370 .797640-1.299031$

C 4.0144871 .2914631 .065141

C 4.8304472 .3971610 .839646

C $4.8377681 .905590-1.519490$

H $3.7199070 .192598-2.145556$

H 3.7045451 .0401702 .074072

H 5.1453073 .0118341 .678495

H $5.1605902 .133799-2.531599$

H $5.8826853 .571158-0.634959$

F $1.820809-0.980548-1.887747$

F $3.418545-2.313933-1.265667$

F $1.409773-2.550160-0.453152$

SCF Energy $\left(P C M / m P W 1 P W 91 / 6-31+G^{\star *}\right)=-1524.12188469$

R-MPTA-3_010

B3LYP/6-31G* Geometry

O $-2.014031-0.131712-0.044291$

C $-4.223752-0.187845-1.105270$

C $-2.577469-1.994100-1.501739$

C $-3.831358-1.549884-1.603329$

C -1.486384-1.201148 -0.825699

C $-2.9846590 .638117-0.747190$

C -3.3492451 .8139190 .112556$

C $-4.499741 \quad 1.8305530 .792395$

C -5.4222660 .6679610 .826042$

O $-5.143456-0.376717-0.000548$

H $-2.5488340 .995409-1.695969$

H $-4.7743100 .352130-1.889245$

C $-0.642378-2.0892990 .105305$

C $-1.379280-2.5798421 .341164$

$\begin{array}{llll}\text { O } & -6.356076 & 0.595667 & 1.592081\end{array}$

H $-0.809664-0.793407-1.596192$

O $0.501057-1.3289180 .585350$

H -2.295381-2.957769-1.921818

H $-4.608419-2.151594-2.067206$

H -2.634097 2.6297280 .182724

H $-4.791666 \quad 2.6583801 .430250$

H $-0.259341-2.922805-0.490891$

H $-0.715898-3.1993001 .951200$

H -2.247627 -3.1779931 .047860$

H $-1.729044-1.7353401 .938791$

C $1.549462-1.225923-0.240582$

O $1.620449-1.728040-1.339876$

C $2.648990-0.301674 \quad 0.359687$

C $4.033429-0.816968-0.124433$

O $2.568473-0.4649881 .761340$

C 3.3950490 .3418012 .605022

H 3.4250581 .3849772 .273499

H $4.414911-0.0509092 .662314$

H 2.9323260 .2904123 .593253

C $2.3203861 .134874-0.087309$

C $1.564813 \quad 3.746754-0.798775$

C $3.0141361 .826149-1.088609$

C 1.2333301 .7613950 .543204

C 0.8635513 .0585790 .193481

C $2.6361393 .124349-1.437860$

H $3.8474921 .366918-1.604560$

H 0.6809481 .2231491 .305729

H 0.0289543 .5336120 .703034

H $3.1886133 .648426-2.212907$

H $1.2786224 .759162-1.070814$

F $4.166324-0.873333-1.460781$

F $5.022472-0.008224 \quad 0.330524$
F $4.258925-2.047566 \quad 0.365827$

SCF Energy $\left(\mathrm{PCM} / \mathrm{mPW} 1 \mathrm{PW} 91 / 6-31+\mathrm{G}^{* *}\right)=-1524.12213462$

R-MPTA-3 012

B3LYP/6-31G* Geometry

O $-2.9813741 .177323-0.277162$

C $-4.399443-0.735210-0.869426$

C $-1.935783-0.970668-0.731328$

C $-3.136801-1.545850-0.812375$

C $-1.7604660 .523040-0.612640$

C $-4.0833450 .748669-1.073908$

C $-5.2629921 .609776-0.724136$

C $-6.245018 \quad 1.1382310 .049841$

C $-6.183684-0.2001360 .689719$

O $-5.153768-1.0156340 .336651$

H -3.831966 $0.881034-2.140383$

H -5.020121 - $1.065115-1.715222$

C -0.7680750 .9111330 .499177$

C -0.5945062 .4135740 .663336$

O $-6.967571-0.5535341 .541046$

H - $-1.3724840 .925750-1.567353$

$\begin{array}{llll}0 & 0.495202 & 0.312937 & 0.090810\end{array}$

H - $1.027996-1.566846-0.745060$

H -3.241316 - $2.626812-0.853386$

H -5.275132 $2.630778-1.097539$

H -7.107953 1.7341140 .328450

H -1.077694 0.4520531 .440966

H - 1.5538992 .8685490 .921309

H $-0.2290732 .864054-0.266149$

H $\quad 0.1210552 .6222791 .463318$

C $1.337110-0.0763521 .063621$

$\begin{array}{lllll}\text { O } & 1.152717 & 0.070298 & 2.244765\end{array}$

C $2.641677-0.6918020 .463785$

C $2.271762-1.710432-0.648308$

O $3.193356-1.4029601 .551885$

C $4.533660-1.8920541 .466916$

H $4.585734-2.8341520 .912739$

H $4.840920-2.0640732 .500956$

H $5.208334-1.1622271 .007583$

C $3.5320440 .470376-0.001628$

C $5.1842982 .649289-0.643723$

C $3.9758740 .658665-1.315831$

C 3.9298901 .3831490 .988675

C 4.7482502 .4641430 .670144

C $4.7970501 .744095-1.630546$

H $3.690260-0.027534-2.102815$

H 3.5940881 .2334422 .010449

H 5.0450193 .1614391 .448792

H $5.1347971 .876041-2.654839$

H $5.8229043 .491770-0.894796$

F $1.699584-1.161633-1.739599$

F $3.368862-2.372945-1.081118$

F $1.408999-2.626733-0.162523$

SCF Energy $\left(P C M / m P W 1 P W 91 / 6-31+G^{\star *}\right)=-1524.12219717$

R-MPTA-3 016

B3LYP/6-31 G* Geometry

O $-3.303262-0.854156-0.641564$

C $-4.4819550 .259134 \quad 1.201349$

C -2.017162 -0.0004111 .236736$

C -3.114213 0.5697991 .738017

C -2.062213 $-0.935395 \quad 0.053911$

C $-4.430300-0.9227250 .228809$

C $-5.666117-0.982063-0.622168$

C $-6.4473120 .092559-0.766542$

C $-6.094521 \quad 1.417861-0.197965$

$\begin{array}{llll}O & -5.009594 & 1.478422 & 0.620345\end{array}$

H $-4.358646-1.8390000 .840043$

H -5.155902 -0.0154612 .025984$

C $-0.976545-0.612274-0.988523$

C $-0.987485-1.545487-2.189709$

O $-6.6904342 .431336-0.484397$

H $-1.897348-1.9734260 .399710$

O $0.272882-0.754111-0.256120$

H $-1.0357680 .187823 \quad 1.663994$

$\mathrm{H}-3.057006 \quad 1.2730042 .564792$

H $-5.885526-1.915931-1.133797$ 
H -7.343427 $0.086561-1.378319$

H - $-1.0727280 .428998-1.306410$

H - $1.950856-1.474787-2.701046$

H $-0.831806-2.582870-1.873205$

H $-0.195395-1.264718-2.888759$

C $1.314610-0.009142-0.654241$

O $1.3253680 .720431-1.614404$

C $2.524188-0.197195 \quad 0.319170$

C $2.945791-1.697158 \quad 0.274450$

O 1.9873720 .1145941 .594867

C $2.833760-0.0746342 .734097$

H $2.946483-1.1372632 .973097$

H 2.3243630 .4276153 .559103

H 3.8209550 .3756442 .587264

C $3.6559750 .751679-0.092440$

C $5.6186252 .614261-0.847019$

C $4.6729670 .389189-0.983541$

C 3.6229532 .0622800 .400321

C 4.5988672 .9858680 .029892

C $5.6484991 .315363-1.353828$

H $4.713015-0.609874-1.400419$

H 2.8241552 .3558501 .073255

H $4.557841 \quad 3.9976100 .424154$

H $6.4327221 .016235-2.043823$

H $6.3804903 .332654-1.137091$

F $3.002483-2.145737-1.002753$

F $4.161292-1.8966850 .827155$

F $2.077921-2.4742270 .939710$

SCF Energy $\left(P C M / m P W 1 P W 91 / 6-31+G^{* *}\right)=-1524.12160160$

R-MPTA-3_017

B3LYP/6-31 G* Geometry

O $-2.019676-0.105428-0.003910$

C $-4.217419-0.035278-1.088154$

C $-2.550732-1.765026-1.695792$

C $-3.807125-1.319423-1.752683$

C -1.473673 -1.053326 -0.914298

C $-2.9904180 .744770-0.605965$

C -3.371832 1.7893820 .403174

C -4.5337701 .7108851 .059000$

C -5.4523480 .5537740 .918519$

O $-5.152294-0.372433-0.033656$

H -2.547860 $1.231619-1.492144$

H $-4.7598470 .598797-1.804429$

C $-0.619711-2.040647-0.099335$

C -1.354481-2.684558 1.065335

$\begin{array}{llll}\text { O } & -6.400835 & 0.377261 & 1.648707\end{array}$

H $-0.799039-0.539040-1.620925$

O $0.515015-1.3255490 .470182$

H -2.257008 -2.665188 -2.232708

H $-4.574831-1.862672-2.297354$

H -2.659310 2.5863670 .599712

H -4.8379402 .4423651 .800402$

H $-0.225583-2.791046-0.791159$

H $-2.227704-3.2329760 .698566$

H -1.696869-1.922866 1.768857

H $-0.696470-3.3858451 .586839$

C $1.570339-1.123542-0.329525$

O $1.665309-1.516829-1.472742$

C $2.638026-0.238707 \quad 0.367255$

C $4.048502-0.614559-0.183040$

O $2.619640-0.358057 \quad 1.766292$

C $2.903352-1.6221002 .366527$

H $2.337934-2.4345841 .897892$

H $2.585018-1.5223073 .406258$

H $3.972530-1.8537322 .334853$

C 2.3139191 .2309510 .036605

C $1.7300763 .912894-0.538724$

C $2.2940382 .183897 \quad 1.060427$

C $2.0412191 .629635-1.279481$

C $1.7505242 .964048-1.562035$

C 2.0033733 .5176750 .770916

H 2.5017501 .8756772 .076953

H $2.0594940 .901406-2.082574$

H $1.5434323 .260381-2.586862$

H 1.9925364 .2485081 .575154

H $1.5050624 .952609-0.761246$
F $4.233701-0.256062-1.461824$

F $4.995007-0.008410 \quad 0.557372$

F $4.264211-1.949270-0.103041$

SCF Energy $\left(\mathrm{PCM} / \mathrm{mPW} 1 \mathrm{PW} 91 / 6-31+\mathrm{G}^{* *}\right)=-1524.12619277$

R-MPTA-3_020

B3LYP/6-31 G* Geometry

O $2.611512-0.5651101 .200451$

C $4.0472760 .094295-0.678495$

C $2.775105-2.031675-0.727402$

C $3.495937-1.115760-1.375977$

C $2.422039-1.9001030 .733579$

C $3.881798-0.0278350 .838868$

C 4.0290051 .3069811 .511203

C 3.8887992 .4406070 .817130

C $3.4715662 .455698-0.606897$

O $3.392703 \quad 1.256792-1.248453$

H $4.675115-0.7083191 .194555$

H $5.1205650 .198975-0.893197$

C $0.974607-2.2745451 .073415$

C $0.647873-3.7537890 .898358$

O $3.1388973 .466247-1.182885$

H $3.062840-2.5826311 .324973$

O $\quad 0.125631-1.480516 \quad 0.207410$

H $2.420597-2.918413-1.246277$

H $3.709413-1.215083-2.436850$

H 4.2260191 .3177062 .580233

H 3.9824813 .4209581 .272451

H $0.785435-1.9683842 .105450$

H $1.313169-4.3690821 .514188$

H $0.744947-4.066217-0.145803$

H $-0.380810-3.9391041 .217011$

C -1.072537-1.124889 0.693391

O $-1.492361-1.4592231 .780082$

C -1.891292 $-0.304028-0.349792$

C $-2.947276-1.318008-0.890903$

O $-1.133366 \quad 0.011359-1.494386$

C $-0.1008260 .993773-1.346796$

H $-0.5158521 .980346-1.115472$

H $0.4033691 .030513-2.313531$

H $\quad 0.6245260 .704332-0.582693$

C -2.616279 $0.923506 \quad 0.233978$

C -3.988938 3.2141951 .115789

C $-3.1340501 .849133-0.686749$

C $-2.803946 \quad 1.1555601 .601534$

C -3.4829172 .2967442 .034918$

C $-3.8131642 .984206-0.249872$

H -2.996786 $1.674791-1.748046$

H -2.434201 0.4445322 .328385

H -3.617011 2.4619773 .100692

H $-4.2035933 .689132-0.978915$

H $-4.5170354 .099947 \quad 1.458750$

F $-3.841237-1.6372140 .058883$

F $-3.615714-0.821350-1.942693$

F - $2.347937-2.461076-1.290562$

SCF Energy $\left(\mathrm{PCM} / \mathrm{mPW} 1 \mathrm{PW} 91 / 6-31+\mathrm{G}^{* *}\right)=-1524.12394503$

R-MPTA-3 021

B3LYP/6-31 G* Geometry

$\begin{array}{lllll}\text { O } & 2.121917 & 0.066470 & -0.085555\end{array}$

C $4.4776660 .646747-0.431617$

C 2.8605972 .3241650 .413856

C 4.1277751 .9672100 .196018

C $1.699706 \quad 1.411946 \quad 0.103217$

C $3.225531-0.046509-0.977750$

C $3.471523-1.508498-1.214912$

C $4.490997-2.133606-0.618048$

C $5.361899-1.4656680 .380513$

O $5.194501-0.1264450 .562501$

H $2.9733550 .446076-1.932469$

H $5.1693720 .804515-1.271816$

C 0.6542501 .4097331 .232728

C 1.1219240 .7393352 .513969

O $6.153632-2.0690911 .068399$

H $1.1960941 .770113-0.811638$

O

H 2.6229513 .3081000 .814307 
H 4.9522872 .6275340 .451662

H $2.773951-2.039727-1.857207$

H $4.691759-3.190634-0.758173$

H $0.3414152 .442878 \quad 1.407936$

H $2.014728 \quad 1.2447932 .895302$

H $1.371908-0.3070462 .327666$

H $\quad 0.3404390 .791823 \quad 3.278001$

C -1.387302 $1.314398-0.014820$

O $-1.3497362 .500105-0.249489$

C $-2.4321280 .343379-0.645045$

C $-1.615496-0.754606-1.400741$

O $-3.110248 \quad 1.001783-1.692027$

C $-4.0640202 .010687-1.347259$

H $-4.877621 \quad 1.602707-0.736980$

H $-4.4688102 .352217-2.302411$

H $-3.5903372 .848132-0.830924$

C $-3.391222-0.312657 \quad 0.360312$

C $-5.243647-1.5410292 .080609$

C $-3.232908-0.2338941 .747849$

C $-4.493208-1.012384-0.156838$

C $-5.409503-1.6230220 .696444$

C $-4.154674-0.8443152 .601357$

H -2.3843720 .2878012 .173514$

H -4.627949-1.071167-1.231156

H $-6.255005-2.1625510 .278375$

H - $4.015396-0.7728483 .676600$

H -5.958835 -2.016107 2.746558

F $-1.089760-1.655703-0.559221$

F $-2.385420-1.418648-2.279583$

F $-0.599426-0.193831-2.097760$

SCF Energy $\left(\mathrm{PCM} / \mathrm{mPW} 1 \mathrm{PW} 91 / 6-31+\mathrm{G}^{* *}\right)=-1524.12391179$

R-MPTA-3_023

B3LYP/6-31G* Geometry

O $2.167442-0.2900190 .327292$

C $4.544818-0.892243 \quad 0.353105$

C $2.881375-2.315816-0.805717$

C $4.159017-2.005779-0.579361$

C $1.736355-1.540161-0.199598$

C $3.328909-0.3993931 .143311$

C 3.5882400 .9492021 .751278

C 4.5607361 .7344661 .278309

C $5.3615441 .379144 \quad 0.080394$

O $5.187650 \quad 0.134131-0.442502$

H $3.144582-1.136898 \quad 1.943637$

H $5.292728-1.2511181 .075008$

C $0.636637-1.265527-1.232645$

C $1.007215-0.233942-2.289823$

O $6.1004072 .163298-0.470115$

H $1.277215-2.1374240 .609901$

O $-0.514439-0.801511-0.471902$

H 2.621471-3.160157-1.441654

H $4.966488-2.558485-1.052257$

H $2.940318 \quad 1.2727962 .561886$

H 4.7690652 .7181181 .686099

H $0.357449-2.213070-1.703736$

H $1.906157-0.553074-2.827522$

H $1.209400 \quad 0.732403-1.823166$

H $0.191152-0.129501-3.009497$

C $-1.727683-1.089165-0.969466$

O $-1.938952-1.683062-1.996915$

C $-2.850944-0.491307-0.062719$

C $-2.713833-1.069048 \quad 1.373082$

O $-4.037673-0.992583-0.637951$

C $-5.296739-0.535666-0.139427$

H $-5.586447-1.0692600 .771367$

H $-6.016438-0.757544-0.930719$

H $-5.2950950 .541900 \quad 0.056052$

C $-2.7593001 .039473-0.148425$

C $-2.6253853 .819963-0.517560$

C $-3.0895001 .613016-1.386124$

C -2.3568851 .8730450 .900811$

C -2.2943593 .2556240 .713577$

C $-3.0230242 .992234-1.569616$

H $-3.4037040 .966256-2.200021$

H $-2.085591 \quad 1.4600041 .863967$

H $-1.9840943 .890977 \quad 1.538689$
H $-3.2816253 .419577-2.534612$

H $-2.5734244 .896201-0.657644$

F $-1.541673-0.7848221 .975647$

F $-3.689193-0.5933452 .182797$

F -2.832271-2.408772 1.336072

SCF Energy $\left(\mathrm{PCM} / \mathrm{mPW} 1 \mathrm{PW} 91 / 6-31+\mathrm{G}^{* *}\right)=-1524.12276734$

R-MPTA-3_024

B3LYP/6-31 G* Geometry

O $2.183323-0.2655410 .310692$

C $4.555926-0.8857100 .245060$

C $2.853913-2.218389-0.966097$

C $4.138759-1.932009-0.749852$

C $1.730524-1.478021-0.280426$

C $3.362447-0.4322211 .091439$

C 3.6469200 .8745551 .774503

C 4.6194801 .6777111 .332672

C $5.394845 \quad 1.389406 \quad 0.100711$

O $5.194800 \quad 0.182101-0.496779$

H $3.189115-1.2153531 .849844$

H $5.314124-1.2990640 .925983$

C $0.603247-1.133701-1.261436$

C $0.945120-0.034107-2.257436$

O $6.1343552 .197380-0.412969$

H $1.291468-2.1238250 .502689$

O $-0.524624-0.717973-0.436735$

H $2.572925-3.016370-1.651038$

H $4.930666-2.456371-1.278114$

H 3.0169281 .1541462 .615028

H 4.8455532 .6322421 .796363

H $0.304944-2.047176-1.785074$

H $1.822112-0.320653-2.847296$

H $1.1712560 .896415-1.732787$

H $\quad 0.1053700 .125313-2.938759$

C $-1.753923-0.966399-0.908473$

O $-2.000597-1.497579-1.967444$

C -2.854528 -0.4241160 .054627$

C $-2.546824-0.873722 \quad 1.509677$

O $-4.127067-0.888806-0.332019$

C $-4.416272-2.291813-0.331155$

H $-3.623439-2.876157-0.803820$

H $-5.330975-2.388718-0.919797$

H $-4.601267-2.6599210 .683540$

C $-2.923278 \quad 1.101077-0.133897$

C $-3.1654013 .846173-0.674712$

C $-3.2676951 .549079-1.418370$

C $-2.7084992 .042053 \quad 0.878491$

C $-2.831273 \quad 3.4062410 .604725$

C $-3.3849302 .910416-1.687415$

H $-3.4533620 .821599-2.201772$

H $-2.449713 \quad 1.733513 \quad 1.882525$

H -2.6648844 .1246891 .402759$

H -3.651274 $3.238885-2.688330$

H $-3.2571754 .909006-0.881421$

F - $1.465588-0.2852722 .054183$

F $-3.595517-0.6221842 .312867$

$F-2.317255-2.2111961 .545804$

SCF Energy $\left(P C M / m P W 1 P W 91 / 6-31+G^{* *}\right)=-1524.12207298$

R-MPTA-3 025

B3LYP/6-31G* Geometry

O $-2.447648 \quad 1.1702900 .931628$

C -3.413576 $0.535341-1.237654$

C $-1.7731532 .383252-1.066945$

C $-2.4704941 .541888-1.830688$

C -1.8199792 .3500640 .440017$

C $-3.648266 \quad 0.822748 \quad 0.248136$

C $-4.232289-0.3729540 .944778$

C $-4.118251-1.593106 \quad 0.412372$

C $-3.344439-1.856406-0.826945$

O $-2.874075-0.779425-1.512144$

H -4.3568851 .6676100 .302758$

H $-4.3881170 .591978-1.744607$

C $-0.4366992 .381807 \quad 1.105695$

C 0.3375613 .6810300 .924874

O $-3.084381-2.973672-1.210162$

H $-2.367358 \quad 3.2400570 .807596$ 
$\begin{array}{lllll}O & 0.312329 & 1.277256 & 0.535453\end{array}$

H -1.135652 $3.136822-1.523248$

H -2.385730 $1.563184-2.913878$

H $-4.717228-0.2153371 .904936$

H $-4.516577-2.4804250 .893350$

H -0.5756422 .1735912 .169055$

H $-0.2362934 .526601 \quad 1.320358$

H $0.5652933 .875512-0.127829$

H 1.2809653 .6235261 .474671

C 1.0867990 .5553151 .365036

O 1.2882070 .8205052 .525886

C $1.723154-0.6710040 .645470$

C $0.665721-1.393362-0.251881$

O $2.222784-1.5661851 .601978$

C $1.331113-2.2107672 .515755$

H $0.642544-1.5025992 .983438$

H $1.978158-2.6386463 .284381$

H $0.770356-3.0135312 .026682$

C $2.928252-0.204970-0.196983$

C $5.1573650 .611203-1.699771$

C $4.184630-0.7808320 .026014$

C $2.7989490 .786918-1.180215$

C $3.9074661 .188631-1.925060$

C $5.289944-0.374149-0.721978$

H $4.289625-1.5446610 .785098$

H $1.8351491 .241293-1.371595$

H $3.7896121 .956099-2.685537$

H $6.257595-0.832712-0.536398$

H $6.0195710 .926831-2.281215$

F $0.361336-0.725965-1.374181$

F $1.127416-2.604863-0.604339$

F $-0.489859-1.5768630 .435266$

SCF Energy $\left(\mathrm{PCM} / \mathrm{mPW} 1 \mathrm{PW} 1 / 6-31+\mathrm{G}^{* *}\right)=-1524.12171574$

R-MPTA-3 033

B3LYP/6-31 G* Geometry

O -2.105163 -1.125058-1.152437

$\begin{array}{llll}\text { C }-3.602833 & -0.738929 & 0.760994\end{array}$

C $-2.043364-2.6635580 .727049$

C $-2.884346-1.886148 \quad 1.409765$

C $-1.709421-2.424044-0.724529$

C $-3.428896-0.775503-0.760694$

C $-3.7490430 .556644-1.375762$

C $-3.7637071 .667567-0.633249$

C $-3.371031 \quad 1.6774970 .797577$

O -3.1279120 .4777351 .388157$

H $-4.132962-1.536208-1.142308$

H $-4.680137-0.8031170 .973276$

C $-0.214901-2.538319-1.052879$

C $0.359723-3.944048-0.917757$

O $-3.2070472 .700046 \quad 1.425388$

H -2.222359 -3.185937-1.344117

O $0.471331-1.626435-0.157347$

H -1.571060 -3.515367 1.210154

H $-3.082546-2.0599952 .464073$

H $-3.9363410 .587792-2.446135$

H -3.981988 2.645266 -1.049601

H $-0.070585-2.175201-2.073690$

H - $0.182788-4.642995-1.564095$

H $\quad 0.303184-4.308348 \quad 0.113137$

H $1.408103-3.939329-1.226526$

C $1.578053-1.030746-0.627953$

O $2.101126-1.278387-1.691984$

$\begin{array}{llll}\text { C } 2.053655 & 0.091601 & 0.331641\end{array}$

$\begin{array}{llll}\text { C } 3.605879 & 0.213568 & 0.249552\end{array}$

$\begin{array}{llll}\text { O } & 1.666392 & -0.125515 & 1.663728\end{array}$

C $2.150720-1.2697392 .363441$

H $2.059627-2.185391 \quad 1.769247$

H $1.514009-1.3544413 .246753$

H $3.191279-1.1412962 .678136$

C $1.3702421 .393514-0.132808$

C $0.0838563 .740563-0.965587$

C $0.599108 \quad 2.133847 \quad 0.769684$

C $1.4822451 .828467-1.461261$

C $0.8467813 .000200-1.869791$

C - $0.042033 \quad 3.3013780 .352421$

H 0.4934661 .7903351 .790279
H $2.0571501 .252251-2.176336$

H $0.9459323 .328803-2.900987$

H $-0.656508 \quad 3.851738 \quad 1.057913$

H $-0.4153604 .650750-1.287743$

F $4.0533910 .690551-0.921087$

F $4.044796 \quad 1.031657 \quad 1.223190$

F $4.195809-0.9934590 .438499$

SCF Energy $\left(\mathrm{PCM} / \mathrm{mPW} 1 \mathrm{PW} 91 / 6-31+\mathrm{G}^{\star \star}\right)=-1524.12366230$

R-MPTA-3_035

B3LYP/6-31G* Geometry

O $3.068184-1.048299-0.658976$

C $4.1875501 .117011-0.379379$

C $1.7143160 .952689-0.414264$

C $2.8093781 .670711-0.157950$

C $1.775702-0.484697-0.867204$

C $4.121982-0.213879-1.133156$

C $5.407778-0.978534-1.000962$

C $6.270653-0.697290-0.019965$

C $5.972033 \quad 0.291694 \quad 1.046372$

$\begin{array}{lllll}\text { O } & 4.837459 & 1.030808 & 0.913443\end{array}$

H $3.9424530 .026154-2.195481$

H $4.7804911 .816959-0.986114$

C $0.798102-1.390336-0.095687$

C $0.891957-2.856853-0.491721$

O $6.656471 \quad 0.4117292 .036871$

H $1.513271-0.546106-1.940415$

O $-0.522505-0.873746-0.428455$

H $0.7243861 .386995-0.304580$

H 2.7403512 .6915370 .208655

H $5.596669-1.780488-1.710287$

H $7.205310-1.2331640 .108683$

H $0.955734-1.2690680 .978882$

H $1.899107-3.228801-0.289314$

H $\quad 0.677014-2.981721-1.558709$

H $0.175048-3.4464510 .085492$

C -1.500849-1.081338 0.466786

O $-1.361602-1.6352431 .530451$

C -2.840238 $-0.481691-0.057656$

C $-4.002958-1.0046660 .836866$

O -2.965502 -1.031608 -1.357938

C $-4.158497-0.773369-2.105910$

H $-4.988940-1.396260-1.759818$

H -3.912071-1.040716-3.135988

H $-4.4509960 .280847-2.062723$

C $-2.7342791 .053983-0.070809$

C $-2.4027823 .844128-0.182019$

C $-2.2070871 .666516-1.217300$

C -3.0784351 .8554721 .026169$

C $-2.915038 \quad 3.2405990 .965787$

C $-2.0474373 .050445-1.274296$

H -1.920567 $1.047916-2.060294$

H $-3.473961 \quad 1.4111551 .930641$

H -3.191536 3.8464061 .824271

H -1.644964 $3.507533-2.174405$

H -2.279794 $4.922823-0.225286$

F -3.828276 $-0.768547 \quad 2.149454$

F $-5.168220-0.4075780 .485667$

F - $4.161078-2.3270130 .669479$

SCF Energy $\left(\mathrm{PCM} / \mathrm{mPW} 1 \mathrm{PW} 91 / 6-31+\mathrm{G}^{* *}\right)=-1524.12143474$

R-MPTA-3_042

B3LYP/6-31 G* Geometry

O $3.086584-1.232286-0.319008$

C $4.1448320 .918586-0.844675$

C $1.6814590 .657933-0.919630$

C $2.7464501 .461304-0.915153$

C $1.796741-0.839349-0.779610$

C $4.148513-0.600957-1.031964$

C $5.440236-1.204217-0.559977$

C $6.237850-0.5362870 .278848$

C $5.856767 \quad 0.7703390 .872138$

O 4.7193251 .3580380 .411752

H $4.022752-0.793297-2.111389$

H $4.7588671 .356479-1.645058$

C $0.799777-1.411096 \quad 0.245067$

C $0.915381-2.916784 \quad 0.430294$ 
O $\quad 6.477467 \quad 1.284247 \quad 1.774596$

H $1.588494-1.318165-1.755202$

O $-0.510779-1.071398-0.294203$

H $0.6771781 .059500-1.023360$

H $2.6375792 .540982-0.974233$

H $5.687396-2.206447-0.901158$

H $7.174390-0.9439210 .644963$

H $0.921403-0.8958601 .201098$

H $1.916680-3.1658770 .790016$

H $0.745752-3.437952-0.518852$

H $0.181736-3.2614551 .163807$

C - $1.509261-0.8982440 .587806$

O $-1.415591-1.0545091 .783798$

C $-2.790124-0.384180-0.121632$

C $-4.040401-0.8851460 .664105$

O $-2.865287-0.789882-1.465183$

C $-2.965498-2.181055-1.771407$

H -2.228675 -2.776765 -1.222042

H $-2.756200-2.254062-2.840791$

H -3.969803 -2.567070 -1.571751

C $-2.7524191 .155919-0.119673$

C $-2.7202653 .959642-0.138840$

C $-3.0935741 .857652-1.281679$

C $-2.399147 \quad 1.867526 \quad 1.035399$

C -2.3823143 .2620021 .021544$

C $-3.0762103 .252909-1.287843$

H $-3.3683971 .308435-2.173183$

H -2.1393701 .3363801 .944364$

H -2.1044373 .8012911 .922845$

H $-3.3438723 .786470-2.195968$

H $-2.7064995 .046201-0.146949$

F $-4.178247-0.3023891 .862556$

F $-5.151404-0.630622-0.052462$

$F-3.988292-2.2233810 .867697$

SCF Energy $\left(\mathrm{PCM} / \mathrm{mPW} 1 \mathrm{PW} 91 / 6-31+\mathrm{G}^{* *}\right)=-1524.12591301$

R-MPTA-3 043

B3LYP/6-31 G* Geometry

O $2.111969-0.3052530 .320960$

C $4.501825-0.8501510 .393920$

C $2.877984-2.377167-0.688425$

C $4.146540-2.023844-0.475010$

C $1.713522-1.595957-0.130314$

C $3.270978-0.3392351 .149070$

C 3.4957761 .0478051 .678783

C 4.4540061 .8257691 .166177

C $5.2704861 .419483-0.004847$

O $5.126450 \quad 0.142631-0.456203$

H $3.096559-1.0326551 .989650$

H $5.252660-1.1509031 .138919$

C $0.606325-1.401779-1.173198$

C $0.974993-0.467819-2.318537$

O $\begin{array}{llll}5.995461 & 2.186382 & -0.596081\end{array}$

H $1.267642-2.1537490 .713898$

O $-0.523170-0.871800-0.420787$

H $2.641542-3.262537-1.275929$

H $4.969343-2.582849-0.912662$

H $2.836410 \quad 1.4023812 .466933$

H 4.6386422 .8354311 .517959

H $0.309546-2.382427-1.559517$

H $1.847231-0.860825-2.851242$

H $1.2248740 .524944-1.938425$

H $0.143219-0.393561-3.022631$

C -1.751439-1.046882 -0.932916

O $-1.995165-1.622029-1.966918$

C $-2.845099-0.392128-0.036078$

C $-2.607828-0.7535721 .463773$

O $-4.112815-0.839893-0.434112$

C $-4.454060-2.223555-0.316829$

H $-3.677051-2.872533-0.727720$

H $-5.364375-2.339778-0.908458$

H $-4.662536-2.4963360 .722968$

C -2.827512 $1.138003-0.235385$

C $-2.855823 \quad 3.919838-0.619928$

C $-1.6571731 .891427-0.056865$

C $-4.0086571 .791234-0.607413$

C $-4.0201213 .173309-0.797637$
C $-1.6768413 .272803-0.248690$

H $-0.731441 \quad 1.407500 \quad 0.228564$

H $-4.9121151 .212959-0.749383$

H $-4.9453493 .664316-1.087229$

H $-0.7620583 .842415-0.108738$

H $-2.8657864 .995960-0.771161$

F $-1.577332-0.1012752 .025391$

F $-3.708355-0.4750202 .183211$

F $-2.356101-2.081877 \quad 1.602667$

SCF Energy $\left(\mathrm{PCM} / \mathrm{mPW} 1 \mathrm{PW} 91 / 6-31+\mathrm{G}^{* *}\right)=-1524.12500050$

R-MPTA-3 044

B3LYP/6-31G* Geometry

$\begin{array}{lllll}\text { O } & 2.245679 & 0.183410 & 0.079023\end{array}$

C $4.4551640 .478995 \quad 1.103209$

C $2.854216-1.1849401 .996767$

C $4.094633-0.6948581 .968475$

C $1.746859-0.6483061 .124951$

C 3.1980821 .1424260 .533423

C $3.5374532 .037208-0.624116$

C $4.6894831 .888373-1.285028$

C $5.6382260 .783890-0.995038$

$\begin{array}{lllll}\text { O } & 5.378394 & 0.005810 & 0.091776\end{array}$

H $2.751931 \quad 1.7410791 .346052$

H $4.991444 \quad 1.2316221 .699144$

C $0.944976-1.7875820 .470412$

C $1.726543-2.600237-0.549351$

O $6.5758780 .520583-1.712341$

H $1.045925-0.0691331 .750756$

O $-0.188741-1.218982-0.241778$

H $2.594983-1.9985832 .671634$

H $4.883687-1.1191292 .583522$

H $2.8070802 .786619-0.918763$

H $4.9643062 .511346-2.129824$

H $0.550658-2.4192631 .271856$

H $2.579008-3.088866-0.067286$

H $2.104222-1.955857-1.346180$

H $1.082790-3.368891-0.985783$

C $-1.282572-0.9274540 .472163$

O $-1.393020-1.1174621 .665278$

C $-2.426931-0.379035-0.434281$

C $-3.449020-1.554827-0.529123$

O $-2.014485-0.205922-1.772025$

C -1.108706 $0.863739-2.053763$

H -1.549634 $1.837933-1.814499$

H $-0.9276500 .805190-3.129151$

H $-0.1602560 .742408-1.522213$

C $-3.1379280 .867494 \quad 0.126150$

C $-4.535013 \quad 3.1522550 .985198$

C -3.0339341 .3065581 .451242$

C $-3.9621031 .580397-0.759993$

C $-4.6528192 .712978-0.334697$

C -3.7261862 .4443101 .872539$

H -2.428703 0.7554492 .158998

H -4.056863 $1.240220-1.785178$

H -5.284818 $3.250641-1.036392$

H -3.632943 2.7710682 .904832

H $-5.072937 \quad 4.035673 \quad 1.318649$

F -4.040157 -1.774320 0.655926

F -4.407917-1.299753-1.432632

F -2.829082 -2.694519-0.899347

SCF Energy $\left(\mathrm{PCM} / \mathrm{mPW} 1 \mathrm{PW} 91 / 6-31+\mathrm{G}^{\star \star}\right)=-1524.12421095$

R-MPTA-3 047

B3LYP/6-31G* Geometry

O $-2.725794-0.578447-1.229553$

C $-4.042957 \quad 0.158751 \quad 0.701261$

C $-2.714341-1.9298910 .790231$

C $-3.417691-0.9953511 .430803$

C $-2.450136-1.873304-0.693989$

C $-3.987955-0.062400-0.811770$

C $-4.2328401 .218646-1.556859$

C $-4.0487502 .398287-0.956066$

C -3.4876492 .5093240 .413307$

O $-3.3639991 .365091 \quad 1.136794$

H $-4.777405-0.792255-1.061233$

H -5.0980980 .2655420 .991009$ 
C $-1.003662-2.204598-1.083617$

C $-0.640318-3.678978-0.943561$

O -3.0761313 .5531640 .869118$

H -3.090481 - 2.614937-1.209073

O $-0.139730-1.422034-0.222231$

H -2.299173 $-2.773781 \quad 1.335136$

H -3.559378-1.036900 2.507257

H $-4.5315111 .153369-2.600004$

H -4.201172 $3.345168-1.463342$

H $-0.851417-1.872532-2.113088$

H - $1.296827-4.297589-1.565223$

H $-0.718497-4.0152560 .095049$

H $0.389608-3.835802-1.276025$

C $0.971072-0.893779-0.766794$

O $1.285257-1.018100-1.927369$

C $1.769525-0.1040920 .325129$

C $2.332694-1.1494031 .334649$

$\begin{array}{lllll}\text { O } & 0.900743 & 0.683496 & 1.108218\end{array}$

$\begin{array}{llll}\text { C } & 0.046143 & 1.585718 & 0.397425\end{array}$

H $0.6185482 .249503-0.260951$

H -0.4613642 .1774931 .160514$

H $-0.7045651 .039590-0.183252$

C $2.9506270 .700251-0.241905$

C $5.1756292 .190883-1.094814$

C $3.7905610 .208750-1.250809$

C 3.2592291 .9297190 .355419

C $4.3572132 .674169-0.073678$

C $4.8911870 .954484-1.674584$

H $3.585313-0.747803-1.711207$

H 2.6407202 .2934421 .167980

H 4.5754513 .6285690 .398012

H $5.5285420 .561778-2.462380$

H $6.0326232 .768986-1.430395$

F $3.041074-2.0906340 .664127$

F $3.151485-0.5699772 .225347$

F $1.370867-1.7821732 .021941$

SCF Energy $\left(P C M / m P W 1 P W 91 / 6-31+G^{\star \star}\right)=-1524.12301376$

R-MPTA-3_048

B3LYP/6-31 G* Geometry

O $-2.9816611 .192333-0.010193$

C $-4.342323-0.538318-1.093101$

C $-1.884456-0.790920-0.887072$

C $-3.072350-1.337314-1.152639$

C $-1.7373950 .639515-0.431146$

C $-4.0376870 .953822-0.938772$

C $-5.2446441 .710858-0.464557$

C -6.2584351 .0739850 .129077$

C $-6.211970-0.3750320 .449813$

O $-5.154537-1.086973-0.024402$

H $-3.7344101 .325429-1.932665$

H $-4.913055-0.669340-2.023904$

C -0.7916860 .7754020 .776889$

C $-0.7100042 .190227 \quad 1.330677$

O $-7.033338-0.9140251 .156163$

H $-1.3194831 .250121-1.253742$

$\begin{array}{lllll}\text { O } & 0.511299 & 0.360370 & 0.266128\end{array}$

H - $0.970075-1.367949-0.986544$

H -3.159255 -2.382680 -1.436655

H $-5.2516122 .790039-0.596314$

H $-7.142458 \quad 1.5893860 .489917$

H $-1.089150 \quad 0.074064 \quad 1.559980$

H -1.6956182 .5033261 .683069$

H -0.3793022 .8930860 .557332$

H -0.0100482 .2239352 .170028$

C $1.369150-0.2055041 .136920$

O $1.158808-0.3285422 .319113$

C $2.668614-0.7107780 .434615$

C $2.239124-1.598359-0.774922$

O $3.326609-1.636027 \quad 1.270942$

C $3.992205-1.1502752 .442040$

H $4.748872-0.3984832 .191446$

H $4.485502-2.0275002 .866086$

H $3.281536-0.7424853 .162827$

C $3.5934610 .417922-0.049556$

C $5.3951002 .384172-0.936399$

C $4.8883480 .067077-0.463462$
C $3.2160611 .764492-0.082682$

C $4.1127842 .740618-0.522751$

C $5.7799221 .042135-0.905037$

H $5.192347-0.973168-0.432534$

H 2.2203262 .0630590 .222166

H $3.8029803 .781964-0.539890$

H $6.7775630 .752101-1.223210$

H $6.0911283 .145070-1.278755$

F $1.771721-0.861548-1.797854$

F $3.264472-2.329545-1.238368$

F $1.259790-2.458844-0.409142$

SCF Energy $\left(\mathrm{PCM} / \mathrm{mPW} 1 \mathrm{PW} 91 / 6-31+\mathrm{G}^{* *}\right)=-1524.12365643$

R-MPTA-3_053

B3LYP/6-31 G* Geometry

O $-2.4021720 .249928 \quad 0.218814$

C $-4.5957821 .161217-0.391536$

C $-2.7235181 .149860-2.013655$

C $-4.026541 \quad 1.306742-1.774626$

C $-1.741296 \quad 0.754533-0.936617$

C -3.4765501 .0792130 .650744$

C $-3.9867070 .528391 \quad 1.951388$

C $-5.124944-0.1707651 .995642$

C $-5.885149-0.5349160 .774039$

O $-5.4742930 .009456-0.404580$

H -3.103678 2.1064430 .805516

H $-5.2180112 .035028-0.149664$

C $-0.767299-0.320024-1.434618$

C $-1.390314-1.694771-1.634905$

O $-6.796584-1.3303450 .782065$

H - $-1.1346361 .634837-0.654171$

O $0.288103-0.393614-0.432024$

H -2.320877 $1.313773-3.011598$

H $-4.7200991 .560388-2.571954$

H -3.3859670 .6932462 .842207$

H $-5.518852-0.5901632 .915548$

H $-0.3094910 .029652-2.364865$

H -2.206816 -1.634518 -2.361928

H $-1.797833-2.070293-0.693633$

H $-0.639143-2.391690-2.016094$

C $1.521554-0.676363-0.874971$

O $1.813231-0.903877-2.028681$

C $2.558856-0.5876150 .276104$

C $3.744304-1.553262-0.030523$

O $2.005693-0.8851341 .532725$

C $1.454737-2.1820341 .766625$

H $0.775173-2.4890450 .964866$

H $0.886189-2.0870282 .694015$

H $2.236524-2.9368231 .896016$

C 3.0732250 .8627860 .343181

C 4.0309043 .4935530 .497157

C 3.1896301 .4996441 .583406

C $3.4438301 .550063-0.820704$

C $3.9181512 .859093-0.740697$

C 3.6661962 .8090421 .656715

H 2.9035980 .9680372 .481997

H $3.3648801 .064722-1.787232$

H $4.2017983 .381106-1.650542$

H 3.7526883 .2927062 .626090

H 4.4014404 .5134130 .557122

F $4.493475-1.156942-1.069453$

F $4.544168-1.6362451 .049164$

F $3.302531-2.803637-0.304284$

SCF Energy $\left(\mathrm{PCM} / \mathrm{mPW} 1 \mathrm{PW} 91 / 6-31+\mathrm{G}^{* *}\right)=-1524.12621139$

R-MPTA-3_057

B3LYP/6-31 G* Geometry

O $-3.302022-0.990802 \quad 0.173452$

C $-4.471189 \quad 1.029658 \quad 0.931763$

C $-1.997800 \quad 0.8819580 .990110$

C $-3.100521 \quad 1.6290881 .063564$

C $-2.037850-0.598258 \quad 0.700439$

C $-4.399063-0.5000960 .941046$

C $-5.655725-1.1083740 .388513$

C $-6.486985-0.387391-0.369821$

C $-6.176990 \quad 0.999811-0.797807$

O $-5.0705091 .586452-0.263991$ 
H $-4.270081-0.8105311 .992359$ H -5.1010781 .3409461 .777804$ C $-0.997777-1.020495-0.352052$ C $-1.081454-2.490652-0.736334$ O $-6.8250161 .587267-1.633338$ H $-1.830274-1.1664261 .626847$ $\begin{array}{lllll} & \text { O } & 0.297665 & -0.767971 & 0.272098\end{array}$ H -1.0166671 .3283131 .128482$ H -3.045434 2.7022301 .226357 H $-5.849198-2.1561090 .604560$ H $-7.399852-0.797174-0.789347$ H $-1.087000-0.382366-1.233799$ H $-2.051732-2.699408-1.192915$ H $-0.961443-3.1278900 .146505$ H $-0.293003-2.733566-1.453823$ C $1.314786-0.466490-0.552490$ O $1.201554-0.296471-1.745451$ C $2.687068-0.4399420 .185177$

C $3.455629-1.670634-0.396376$

O $2.584099-0.7617331 .556775$

C $2.040800 \quad 0.2149932 .441654$

H 2.5791051 .1668612 .381092

H $2.164836-0.2043453 .442625$

H 0.9760050 .3810192 .252149

C $3.5028810 .841093-0.064394$

C $5.1033883 .127238-0.402989$

C $4.637621 \quad 1.0466960 .737251$

C $3.1864431 .789817-1.043602$

C $3.9823522 .925949-1.206451$

C 5.4298542 .1803140 .569730

H 4.8938880 .3130931 .493537

H $2.3336731 .638570-1.692812$

H $3.7214433 .652229-1.971405$

H 6.3037752 .3217451 .199856

H $5.7203274 .012022-0.534672$

F $3.784035-1.478495-1.681137$

F $4.583936-1.9113810 .290112$

F $2.689110-2.781108-0.324680$

SCF Energy $\left(\mathrm{PCM} / \mathrm{mPW} 1 \mathrm{PW} 91 / 6-31+\mathrm{G}^{* *}\right)=-1524.12286405$

R-MPTA-3 058

B3LYP/6-31 G* Geometry

O $-3.1928051 .005558-0.661272$

C $-4.518993-1.037572-0.378301$

C $-2.046289-1.136080-0.505895$

C $-3.201732-1.742930-0.229062$

C $-1.9705850 .315615-0.908085$

C $-4.3411730 .291615-1.115553$

C $-5.5366691 .180964-0.927924$

C $-6.3820730 .978840 \quad 0.087181$

C $-6.139586-0.0456361 .134275$

O $-5.098804-0.9009870 .943724$

H $-4.2199160 .053979-2.186199$

H -5.211228 -1.657466 - 0.966385

C $-0.9006011 .089226-0.114622$

C $-0.7899012 .553280-0.512452$

O $-6.784778-0.1004802 .156431$

H $-1.7129370 .385395-1.981703$

O $0.3458970 .394075-0.409164$

H - $-1.103027-1.672503-0.460343$

H -3.228759 -2.7797600 .095504$

H $-5.6729652 .004199-1.624843$

H $-7.251533 \quad 1.604860 \quad 0.258648$

H - 1.1011320 .9925790 .955169

H $-1.7518103 .048080-0.356690$

H $-0.5139582 .646096-1.568791$

H $-0.034605 \quad 3.0535360 .099487$

$\begin{array}{llll}\text { C } & 1.309845 & 0.416489 & 0.521807\end{array}$

O 1.2510311 .0383881 .559322

C $2.506564-0.5229920 .201117$

C $2.417191-1.144735-1.221003$

O $2.407700-1.663387 \quad 1.042522$

C $2.459279-1.4753132 .461005$

H $2.591503-2.4788172 .871627$

H $1.532376-1.0383062 .843606$

H $3.300547-0.8408172 .756651$

C 3.8407870 .2184360 .331097
C $6.333274 \quad 1.4979620 .465872$

C 3.9481611 .5894490 .074718

C $4.996192-0.509354 \quad 0.642338$

C $6.233670 \quad 0.1282880 .716084$

C 5.1879892 .2254410 .141380

H $3.0662032 .172312-0.171841$

H $4.919626-1.5764640 .820847$

H $7.120882-0.4476910 .964600$

H $5.2553793 .291505-0.056899$

H 7.2977351 .9952010 .522879

F $2.403087-0.177428-2.158987$

F $3.481900-1.931397-1.460881$

F $1.321632-1.909755-1.385876$

SCF Energy $\left(\mathrm{PCM} / \mathrm{mPW} 1 \mathrm{PW} 91 / 6-31+\mathrm{G}^{* *}\right)=-1524.12393329$

R-MPTA-3_071

B3LYP/6-31G* Geometry

O $3.026706-1.917169-1.076527$

C $4.233130 \quad 0.205370-1.093458$

C $1.7647040 .177189-1.347045$

C $2.9004040 .872009-1.297847$

C $1.732788-1.321424-1.228112$

C $4.053075-1.167845-0.424494$

C $3.844118-1.0077681 .060194$

C $4.274740 \quad 0.079241 \quad 1.708018$

C 5.0161581 .1583121 .013764

O $5.1209801 .071095-0.346485$

H $4.951378-1.777986-0.583943$

H $4.7470550 .080308-2.052093$

C $0.784571-1.810191-0.105656$

C $0.711855-3.3253340 .013828$

O $5.5748192 .059421 \quad 1.594670$

H $1.347962-1.739400-2.169327$

O $-0.525410-1.292000-0.482579$

H $\quad 0.8074750 .669207-1.492225$

H $2.913247 \quad 1.953173-1.411642$

H $3.375466-1.8347241 .589425$

H 4.1687800 .2084342 .779836

H $1.058977-1.3575480 .849822$

H $\quad 0.036318-3.6080490 .826348$

H $1.704295-3.7334520 .222053$

H $0.349751-3.768066-0.920821$

C - $1.350219-0.9232970 .512042$

O $-1.110310-1.0359521 .692771$

C $-2.625402-0.245776-0.055894$

C $-3.798290-0.4324820 .954103$

O $-2.986458-0.748433-1.317902$

C $-3.340980-2.125703-1.445416$

H $-4.345142-2.322698-1.057630$

H -2.621172 - $2.782905-0.945351$

H $-3.321159-2.328250-2.518339$

C $-2.325748 \quad 1.253786-0.237914$

C $-1.811548 \quad 3.987691-0.584053$

C $-2.7204191 .901980-1.413705$

C -1.6760951 .9842050 .766825$

C -1.4201823 .3438560 .590673$

C -2.462355 $3.262884-1.582839$

H -3.224512 $1.338118-2.188263$

H -1.3730701 .4943901 .685757$

H -0.9140343 .8983991 .375950$

H -2.773876 $3.755742-2.499874$

H -1.610806 $5.047010-0.719380$

F - - 3.6296280 .2597932 .089639

F $-4.947486-0.0240700 .384585$

F - $3.954595-1.7343481 .292449$

SCF Energy $\left(\mathrm{PCM} / \mathrm{mPW} 1 \mathrm{PW} 91 / 6-31+\mathrm{G}^{\star *}\right)=-1524.12060188$

R-MPTA-3_082

B3LYP/6-31 G* Geometry

O $2.198093-0.101970 \quad 0.316226$

C $4.567728-0.6675270 .586854$

C $2.945297-2.341754-0.251115$

C $4.214276-1.978968-0.056539$

C $1.781732-1.4457650 .098464$

C $3.324179-0.0015321 .182810$

C 3.5657021 .4559331 .452320

C 4.5598402 .1056680 .839220 
C $5.4023551 .471808-0.205255$

O $5.2442320 .135208-0.412065$

H $3.101872-0.5213472 .130493$

H $5.285734-0.8333491 .403044$

C $0.724802-1.434469-1.012971$

C $1.148039-0.705630-2.280864$

O $6.1620142 .101197-0.905661$

H $1.284840-1.8258321 .009911$

O $-0.445686-0.793894-0.423682$

H $2.707064-3.318985-0.667289$

H $5.037207-2.627870-0.344276$

H 2.8894111 .9643322 .134735

H 4.7580623 .1598991 .002299

H $0.446451-2.467484-1.242779$

H $2.044727-1.174193-2.699896$

H $1.3819750 .338838-2.062681$

H $\quad 0.349769-0.759289-3.025498$

C $-1.651560-1.196754-0.859445$

O $-1.824886-1.979518-1.763753$

C $-2.809892-0.570441-0.022660$

C $-2.527329-0.9104841 .474987$

O $-4.006231-1.273540-0.276214$

C -4.637282-1.103722 - 1.549418

H -5.559608 -1.685098 -1.484462

H $-4.014040-1.492655-2.356642$

H $-4.887200-0.053541-1.737876$

C $-2.9699270 .949624-0.191263$

C $-3.4094043 .714801-0.440511$

C $-2.016317 \quad 1.767778-0.806645$

C $-4.148175 \quad 1.5344180 .300068$

C $-4.3646882 .905065 \quad 0.177422$

C $-2.2375653 .141306-0.930797$

$\mathrm{H}-1.0898301 .347843-1.178481$

H $-4.8942020 .906760 \quad 0.774747$

H -5.2822373 .3399330 .564486$

H -1.486280 $3.760712-1.413067$

H -3.578655 $4.783686-0.538468$

F -1.540315 -0.1532531 .982453$

F $-3.617400-0.7169932 .235219$

F -2.162977 -2.205127 1.607318

SCF Energy $\left(\mathrm{PCM} / \mathrm{mPW} 1 \mathrm{PW} 91 / 6-31+\mathrm{G}^{* *}\right)=-1524.12460949$

R-MPTA-3_092

B3LYP/6-31G* Geometry

O $3.026383-1.915017-1.079913$

C $4.2323720 .207818-1.092923$

C $1.7638870 .179645-1.345841$

C $2.8994460 .874621-1.295485$

C $1.732304-1.319208-1.229889$

C $4.052829-1.166823-0.426752$

C $3.844491-1.0098751 .058377$

C 4.2751280 .0758991 .708266

C 5.0160181 .1565601 .015922

O $5.1204011 .072065-0.344538$

H $4.951231-1.776401-0.587809$

H $4.7459100 .084809-2.052036$

C $0.784492-1.810355-0.108103$

C $0.712229-3.3257290 .008624$

O $5.5745882 .056697 \quad 1.598420$

H $1.347234-1.735374-2.171800$

O $-0.525793-1.292034-0.483760$

H $0.8065270 .671776-1.489778$

H $2.9119941 .956019-1.407049$

H $3.376229-1.838021 \quad 1.586091$

H 4.1695540 .2028712 .780387

H $1.058940-1.3593560 .848131$

H $1.704786-3.7339630 .216052$

H $\quad 0.350159-3.766887-0.926778$

H $\quad 0.036833-3.610100 \quad 0.820698$

C $-1.349838-0.9236290 .511615$

O $-1.109011-1.036508 \quad 1.692125$

C $-2.625498-0.246049-0.055233$

C $-3.797297-0.4320310 .956116$

O $-2.988027-0.749214-1.316627$

C $-3.342526-2.126582-1.443164$

H $-2.622358-2.783471-0.943205$

H -3.323392 -2.329726 -2.515987
H $-4.346411-2.323410-1.054578$

C $-2.325801 \quad 1.253360-0.238232$

C $-1.8114783 .987021-0.586176$

C $-2.7217771 .901133-1.413814$

C -1.6747531 .9840850 .765385$

C $-1.418778 \quad 3.343610 \quad 0.588338$

C -2.463662 $3.261914-1.583847$

H -3.226894 $1.337039-2.187538$

H -1.3707191 .4946241 .684181$

H -0.9115353 .8983801 .372749$

H -2.776238 $3.754433-2.500705$

H -1.610694 $5.046244-0.722194$

F -3.627049 0.2604192 .091293

F -4.946997 -0.0232690 .387820$

F -3.953756 -1.7337831 .294933$

SCF Energy $\left(P C M / m P W 1 P W 91 / 6-31+G^{* *}\right)=-1524.12060451$

R-MTPA-4 001

B3LYP/6-31 G* Geometry

O $-2.9413650 .257358 \quad 1.077905$

C $-4.370349-0.939159-0.519020$

C $-1.911522-0.881918-0.812094$

C $-3.115678-1.159981-1.314854$

C $-1.725926-0.2720290 .553365$

C -4.041690 -0.640167 0.946355

C $-5.217895-0.0290261 .652299$

C -6.2051690 .5458210 .958656$

C $-6.1513970 .712642-0.515514$

O $-5.1259290 .110189-1.176305$

H -3.783068 -1.6017331 .421691$

H $-4.995215-1.843682-0.544535$

C -0.7286280 .9069080 .563699$

C -1.104663 $2.056389-0.354129$

O $-6.935565 \quad 1.404690-1.124449$

H -1.331516 -1.0369221 .245564$

O $0.5600340 .401457 \quad 0.113792$

H -1.005102 -1.095674-1.372865

H -3.229085 - $1.571816-2.314220$

H $-5.222743-0.0461702 .739277$

H -7.0657501 .0037151 .435187$

H $-0.6227891 .241761 \quad 1.598126$

H -2.089602 $2.436390-0.071058$

H - $1.145727 \quad 1.732020-1.398190$

H $-0.3724402 .864612-0.267176$

C $1.366915-0.1626481 .029744$

O $1.131542-0.2545592 .207762$

C $2.699176-0.6381100 .367136$

C $2.362764-1.596319-0.808813$

O $3.322222-1.3877951 .387776$

C $4.670327-1.8279851 .207734$

H $5.041043-2.0359182 .214005$

H $5.295825-1.0576340 .744794$

H $4.718401-2.7427090 .608848$

C $3.5042790 .608281-0.026238$

C $4.9891822 .937268-0.531685$

C 3.9251871 .4314331 .030416

C $3.8360540 .963857-1.338404$

C $4.5757682 .122913-1.584902$

C 4.6610262 .5870240 .779953

H $3.673796 \quad 1.1529272 .049741$

H $3.5233890 .353242-2.175992$

H $4.8280662 .385254-2.608664$

H 4.9777663 .2133041 .609453

H $5.5635493 .838037-0.729878$

F $1.611963-2.619714-0.359405$

F $3.488121-2.116751-1.348377$

F $1.682928-1.018076-1.823128$

SCF Energy $\left(P C M / m P W 1 P W 91 / 6-31+G^{\star \star}\right)=-1524.12275718$

R-MTPA-4 002

B3LYP/6-31G* Geometry

O $-2.8859040 .290201 \quad 1.066141$

C $-4.336729-0.878274-0.531964$

C $-1.878740-0.851527-0.835792$

C $-3.088441-1.113075-1.333981$

C $-1.679466-0.2464940 .529932$

C $-3.997927-0.5931140 .933718$ 
C -5.1633390 .0283581 .648524$

C -6.1451410 .6213840 .962465$

C $-6.0942950 .797802-0.510764$

O $-5.0789770 .186096-1.179303$

H $-3.749126-1.5608281 .401823$

H $-4.974701-1.773528-0.560197$

C -0.6735860 .9253930 .536227$

C $-1.0517232 .084298-0.368509$

O $-6.8710291 .504343-1.112153$

H $-1.284881-1.0165771 .216732$

$\begin{array}{lllll}\mathrm{O} & 0.605401 & 0.411407 & 0.064266\end{array}$

H - $0.977393-1.077196-1.399845$

H $-3.211116-1.521276-2.333712$

$\begin{array}{llll}H & -5.164837 & 0.003438 & 2.735354\end{array}$

H -6.9979431 .0871831 .445251$

H -0.5495621 .2518341 .571233$

H -2.029385 $2.470928-0.069786$

$\mathrm{H}-1.1090671 .767374-1.414036$

H - $0.3112582 .885323-0.285585$

C $1.429828-0.1574120 .958140$

O $1.216758-0.2516232 .144671$

C 2.747409-0.641203 0.277573

C $2.394505-1.462305-0.995256$

O $3.505707-1.4315021 .162813$

C $2.978914-2.6664241 .663100$

H $3.627785-2.9270742 .501747$

H $3.032464-3.4582020 .908274$

H $1.953328-2.5597022 .023954$

C 3.6196180 .5966580 .011818

C $5.2647912 .856872-0.247489$

C $4.0890130 .987387-1.246505$

C 3.9896651 .3442791 .140183

C 4.8032732 .4670341 .011298

C $4.9077192 .112296-1.370207$

H $3.8323420 .429646-2.137286$

H 3.6433491 .0329542 .120398

H 5.0787903 .0347601 .895816

H $5.2672022 .401401-2.353948$

H $5.9006913 .731875-0.350893$

F $1.451623-2.396850-0.699521$

F $3.476313-2.111211-1.455444$

F $1.893796-0.730307-2.009685$

SCF Energy $\left(\mathrm{PCM} / \mathrm{mPW} 1 \mathrm{PW} 91 / 6-31+\mathrm{G}^{* *}\right)=-1524.12161477$

R-MTPA-4_004

B3LYP/6-31G* Geometry

O $-2.9820550 .689332-0.851387$

C -4.3880440 .6545301 .160578$

C $-2.027436-0.0902921 .249016$

C $-3.223860-0.0142751 .834677$

C $-1.7731350 .427601-0.142726$

C $-3.931246 \quad 1.424011-0.082317$

C $-5.0931001 .747074-0.977406$

C $-6.2384281 .065185-0.880142$

C $-6.397148-0.102013 \quad 0.023230$

$\begin{array}{llll}\text { O } & -5.379877 & -0.367935 & 0.886765\end{array}$

H -3.4650522 .3597410 .270968$

H -4.8565911 .3749761 .846669$

C $-0.987822-0.567212-1.024698$

C $-1.672986-1.908107-1.220652$

O $-7.352291-0.843405-0.027759$

H $-1.1750741 .354188-0.085465$

O $0.284911-0.830796-0.370028$

H $-1.173816-0.527977 \quad 1.761187$

H -3.394530 -0.4221502 .827587$

H $-4.9593712 .529205-1.720541$

H $-7.0946241 .265572-1.515832$

H $-0.787944-0.077905-1.980958$

H $-1.074476-2.545314-1.878444$

H $-2.654549-1.750081-1.674428$

H $-1.811343-2.424075-0.265881$

C $1.2919250 .027710-0.590328$

O $1.2385981 .001950-1.300920$

$\begin{array}{llll}C & 2.562301 & -0.399897 & 0.215283\end{array}$

C $3.022388-1.786441-0.328960$

O $2.096517-0.532537 \quad 1.547406$

C $3.010970-1.0468902 .521789$
H $2.539111-0.857607 \quad 3.488213$

H $3.978945-0.5362872 .483010$

H $3.158751-2.1247182 .398441$

C 3.6398600 .6817470 .071439

C $5.5043582 .771940-0.145450$

C $3.6191701 .753098 \quad 0.973087$

C $4.5937410 .678416-0.953338$

C $5.5207061 .715779-1.056275$

C 4.5466022 .7887150 .868967

H 2.8683251 .7719411 .756065

H $4.621216-0.124189-1.680414$

H $6.2566641 .695528-1.855358$

H 4.5167493 .6098881 .580070

H $6.2289753 .577401-0.228295$

F $2.220556-2.7736070 .095421$

F $4.276149-2.0921010 .067018$

F $3.009885-1.801522-1.683978$

SCF Energy $\left(\mathrm{PCM} / \mathrm{mPW} 1 \mathrm{PW} 91 / 6-31+\mathrm{G}^{* *}\right)=-1524.12160564$

R-MTPA-4 008

B3LYP/6-31 G* Geometry

O $-2.542134-1.1285721 .040797$

C $-3.327439-0.581180-1.201819$

C $-1.580102-2.298905-0.862823$

C $-2.265001-1.519395-1.700330$

C $-1.767161-2.2521370 .633553$

C $-3.702366-0.9170590 .241343$

$\begin{array}{llll}\text { C } & -4.487143 & 0.200477 & 0.868930\end{array}$

C -4.3764141 .4446990 .394660$

C -3.417615 $1.794612-0.683027$

O $-2.8254270 .773094-1.357546$

H $-4.310728-1.8371740 .218463$

H $-4.231216-0.661413-1.822014$

C $-0.427500-2.1871201 .383728$

C $-0.581122-1.8827522 .868795$

O $-3.1080512 .938712-0.933248$

H -2.268744 -3.1795110 .972410$

O $0.356058-1.1634000 .709075$

H $-0.828474-2.989624-1.239043$

H -2.072289-1.535844 -2.769333

H $-5.107060-0.026741 \quad 1.732416$

H -4.9026412 .2889040 .827713$

H $\quad 0.095910-3.1396691 .243926$

H -1.232554 -2.631143 3.334364

H -1.031498 -0.8981663 .009077$

H $0.393702-1.9162393 .358179$

C $1.685985-1.1747360 .887976$

O $2.271006-1.9107681 .647789$

C $2.398486-0.127017-0.022588$

C $2.062112-0.444990-1.516086$

O $3.785603-0.2033600 .172411$

C $4.523507-1.368118-0.210156$

H $5.499154-1.2467800 .265424$

H $4.657603-1.413774-1.296027$

H $4.059655-2.2862840 .153945$

C $1.973914 \quad 1.310242 \quad 0.352323$

C 1.2808553 .9487291 .027907

C 2.9431292 .1990150 .835411

C 0.6484351 .7544710 .223683

C 0.3101173 .0678140 .550503

C 2.5955063 .5072401 .173785

H 3.9665461 .8639140 .940180

H $-0.126127 \quad 1.091041-0.135308$

H -0.7165303 .3960020 .414553$

H 3.3613214 .1823291 .546974

H $1.0143724 .971341 \quad 1.282201$

F $2.185999-1.781629-1.750986$

F $2.9219550 .189483-2.329108$

F $0.818941-0.110093-1.891787$

SCF Energy $\left(P C M / m P W 1 P W 91 / 6-31+G^{\star *}\right)=-1524.12054222$

R-MTPA-4_009

B3LYP/6-31 G* Geometry

O $2.808134-0.4900510 .926521$

C $4.1176451 .207396-0.265647$

C $1.7446170 .800596-0.844388$

C $2.9119671 .371422-1.146992$ 
C $1.564436-0.0910630 .356364$

C 3.7294100 .5878321 .079227

C 4.9361790 .0576091 .799474

C $6.062445-0.2116691 .132164$

C $6.156436-0.110277-0.345975$

O $5.0961050 .432591-1.004373$

H 3.2592671 .3850791 .679764

H $4.5731352 .187770-0.064681$

C $0.830444-1.4098480 .031811$

C $1.498375-2.251310-1.041591$

O $7.095891-0.543738-0.973380$

H $0.9641930 .437857 \quad 1.118126$

O $-0.502203-1.069045-0.448590$

H $0.8626140 .965379-1.458450$

H $3.0268281 .986104-2.035846$

H $4.851471-0.1251342 .867737$

H $6.950534-0.6037721 .616782$

H $0.726686-1.9667040 .965571$

H $2.527007-2.467126-0.741476$

H $1.520957-1.726436-2.001249$

H $0.962901-3.196787-1.171794$

C $-1.479414-0.9482640 .465857$

O $-1.344922-1.1138261 .657498$

C $-2.804199-0.485703-0.197032$

C $-4.004700-1.0777620 .604354$

O $-2.894971-0.854236-1.549123$

C $-2.928615-2.238936-1.898234$

H -2.142223 -2.808933 -1.392452

H -2.750228 -2.266020 -2.975156

H -3.903406 -2.685911-1.680463

C -2.846239 $1.053989-0.147770$

C $-2.953973 \quad 3.855356-0.080779$

C $-2.5321001 .746556 \quad 1.029985$

C $-3.217001 \quad 1.773352-1.289253$

C -3.269273 $3.167371-1.252599$

C -2.5859653 .1397831 .059486$

H -2.249959 1.2000871 .922975

H $-3.4607301 .238725-2.198368$

H -3.559440 $3.714557-2.145539$

H -2.341131 3.6644701 .978985

H -2.995398 $4.940962-0.055506$

F -3.869869 -2.415248 0.769884

F $-5.145256-0.869146-0.079368$

F - $4.148233-0.5363051 .821911$

SCF Energy $\left(\mathrm{PCM} / \mathrm{mPW} 1 \mathrm{PW} 91 / 6-31+\mathrm{G}^{* *}\right)=-1524.12606936$

R-MTPA-4 010

B3LYP/6-31 G* Geometry

O $3.049011-0.693148-0.894363$

C $4.348627-0.3401291 .158727$

C $1.873286-0.3347841 .210127$

C $3.028735-0.0852561 .828359$

C $1.804101-0.822875-0.214078$

C $4.160650-1.150155-0.126964$

C $5.380729-1.072447-0.999190$

C $6.269838-0.087298-0.840028$

C $6.058131 \quad 1.031431 \quad 0.112652$

$\begin{array}{lllll}\text { O } & 4.992356 & 0.943239 & 0.954149\end{array}$

H $3.986671-2.1972440 .174664$

H $5.001877-0.9203241 .826473$

C $0.785769-0.050231-1.075599$

C $1.0554711 .441183-1.195500$

O 6.7495422 .0245200 .119569

H $1.489765-1.883057-0.213549$

O $-0.496414-0.258793-0.419440$

H $0.921023-0.2086601 .718101$

H 3.0542070 .2940942 .846534

H $5.498763-1.823425-1.776361$

H $7.1579120 .004715-1.456594$

H $0.745441-0.513988-2.063109$

H $2.0497891 .598278-1.622085$

H $1.0158551 .929786-0.217267$

H $0.3129311 .904645-1.851958$

C $-1.564776-0.534750-1.186439$

O $-1.559806-0.627264-2.387300$

C $-2.857507-0.645853-0.317259$

C $-2.585205-1.5414910 .922205$
O $-3.760189-1.324424-1.165167$

C $-5.141473-1.399222-0.805201$

H $-5.670083-1.609170-1.737898$

H $-5.508741-0.456224-0.387346$

H $-5.328910-2.208915-0.093368$

C -3.312785 $0.784477 \quad 0.011954$

C -4.1748183 .4292600 .401922$

C $-3.6519661 .595533-1.083151$

C $-3.4149381 .312047 \quad 1.304496$

C -3.8442382 .6278331 .493478$

C $-4.0785362 .907241-0.889897$

H $-3.5798661 .185531-2.086049$

H -3.1630690 .7138142 .170654$

H $-3.919799 \quad 3.0227752 .502911$

H $-4.3346813 .521224-1.748954$

H $-4.506446 \quad 4.4524910 .555486$

F $-2.115914-2.7388340 .528277$

F $-3.725958-1.7564601 .618123$

F - $1.697375-1.0276491 .802094$

SCF Energy $\left(\mathrm{PCM} / \mathrm{mPW} 1 \mathrm{PW} 91 / 6-31+\mathrm{G}^{* *}\right)=-1524.12239529$

R-MTPA-4 011

B3LYP/6-31G* Geometry

O $2.982396-0.648645-0.877623$

C $4.309195-0.2895791 .157130$

C $1.834878-0.2485391 .236219$

C $3.000644-0.0103531 .839277$

C $1.742878-0.747201-0.182976$

C $4.096013-1.112368-0.116325$

C $5.307460-1.061684-1.002632$

C $6.209906-0.085316-0.866172$

C $6.022028 \quad 1.0483570 .073869$

$\begin{array}{lllll}\text { O } & 4.965465 & 0.983203 & 0.929025\end{array}$

H $3.910547-2.1531620 .199671$

H $4.963294-0.8697801 .824002$

C $0.7316580 .040754-1.040318$

C $1.0282671 .526105-1.167279$

$\begin{array}{lllll}\text { O } & 6.724456 & 2.033468 & 0.059294\end{array}$

H $1.406441-1.800721-0.170485$

O $-0.551200-0.132448-0.372962$

H $0.890124-0.103401 \quad 1.753321$

H 3.0426310 .3777902 .853542

H $5.408209-1.824509-1.770638$

H $7.092315-0.012237-1.493331$

H $0.672065-0.428096-2.024266$

H $2.0238221 .662113-1.598175$

H $1.0018272 .019217-0.191001$

H $0.2920862 .002661-1.821597$

C $-1.606351-0.544183-1.093176$

O $-1.598711-0.780254-2.278246$

C $-2.889332-0.620499-0.209342$

C $-2.559993-1.3510981 .123559$

O $-3.921907-1.298474-0.885650$

C -3.750999-2.667848 -1.270973

H $-4.560185-2.861753-1.977992$

H -3.855022 -3.340569-0.413032

H -2.793029 $-2.837741-1.768509$

C -3.416666 $0.812635-0.026709$

C -4.4687923 .4133460 .095719$

C $-3.7069001 .522239-1.201874$

C -3.6712861 .4144091 .210343$

C -4.1941502 .7083791 .266178$

C $-4.2254732 .813315-1.141224$

H $-3.5337011 .051255-2.164040$

H $-3.4751130 .891828 \quad 2.137194$

H -4.3883353 .1611942 .234602$

H $-4.4425883 .348367-2.061633$

H $-4.873460 \quad 4.4205300 .145827$

F $-1.895705-2.506938 \quad 0.873024$

F $-3.689120-1.667238 \quad 1.780186$

$F-1.786711-0.6394931 .970799$

SCF Energy $\left(\mathrm{PCM} / \mathrm{mPW} 1 \mathrm{PW} 91 / 6-31+\mathrm{G}^{* *}\right)=-1524.12186610$

R-MTPA-4_012

B3LYP/6-31G* Geometry

O $2.959774-1.2037050 .084359$

C 3.4321290 .9709441 .108754 
C $1.807487-0.5909122 .136251$

C 2.3361750 .6328062 .078934

C $2.217656-1.697257 \quad 1.195691$

C $3.992898-0.2961810 .459334$

C $4.7878050 .033160-0.771953$

C $4.6158931 .199905-1.400812$

C $3.5680222 .171550-1.000205$

O $2.891325 \quad 1.921546 \quad 0.155633$

H $4.648717-0.7788041 .204512$

H 4.2563661 .4774191 .631146

C $1.016689-2.483050 \quad 0.645527$

C $1.405778-3.582724-0.332652$

O $3.2533293 .117302-1.685877$

H $2.835245-2.4311021 .750382$

O $0.139681-1.575629-0.071835$

H $1.038552-0.8333952 .865666$

H 1.9967471 .4252262 .740637

H $5.481507-0.715728-1.145664$

H $5.1656431 .469171-2.296620$

H $0.461511-2.8891181 .495349$

H $2.055556-4.3139260 .161020$

H $1.936057-3.163613-1.190507$

H $0.508437-4.097584-0.687119$

C $-1.002571-1.1968130 .518664$

O $-1.344359-1.5327651 .632800$

C $-1.879460-0.345127-0.451542$

C $-2.972579-1.340334-0.954372$

O $-1.1986450 .001984-1.633962$

C $-0.1129370 .931794-1.527643$

H $0.1632471 .167351-2.557320$

H $0.7423160 .488468-1.013274$

H $-0.4146971 .852677-1.018389$

C $-2.571026 \quad 0.867473 \quad 0.202535$

C -3.9194793 .1191291 .213543$

C -2.7711721 .0155541 .580215$

C $-3.0702581 .853967-0.663123$

C $-3.7355942 .970675-0.162104$

C -3.4368042 .1379842 .078013$

H -2.419463 0.2535582 .262774

H -2.931412 $1.739886-1.732250$

H -4.110536 $3.724194-0.849488$

H -3.580385 2.238042 3.150701

H -4.4374683 .9900561 .606294$

F - $2.408169-2.467447-1.437958$

F $-3.711803-0.801066-1.934567$

F -3.797439-1.694587 0.045288

SCF Energy $\left(P C M / m P W 1 P W 91 / 6-31+G^{* *}\right)=-1524.12370629$

\section{R-MTPA-4 014}

B3LYP/6-31 G* Geometry

O $3.147719-1.078446 \quad 0.561814$

C $4.715736 \quad 0.8074190 .678968$

C 2.2738441 .1959990 .539925

C 3.5134811 .6844810 .473700

C $1.991140-0.2691730 .754661$

C $4.304626-0.5651781 .218340$

C $5.409428-1.5682521 .047911$

C $6.390254-1.3574790 .164946$

C $6.393308-0.197709-0.761725$

O $5.4358770 .751568-0.578718$

H $4.083979-0.4360252 .291943$

H 5.3942791 .2656041 .413100

C $0.932121-0.827675-0.214247$

C $1.276385-0.667883-1.687386$

O $7.162347-0.105460-1.691411$

H $1.602651-0.4149901 .779240$

O $-0.273709-0.0832750 .115640$

H $1.404478 \quad 1.841076 \quad 0.440413$

H $3.6964492 .738646 \quad 0.282663$

H $5.366836-2.4771981 .642781$

H $7.199396-2.0634550 .009394$

H $\quad 0.761914-1.8803370 .025701$

H $2.248265-1.129738-1.881979$

H $1.3338690 .389127-1.964435$

H $0.520904-1.158500-2.306386$

C $-1.455099-0.661191-0.142305$

O $-1.606295-1.760746-0.614479$
C -2.627686 0.2896950 .267238

C $-2.4865271 .602226-0.563196$

O -2.3880030 .5586861 .637658$

C -3.2423551 .5063552 .286140$

H -3.038324 1.3955683 .353228

H -4.3007031 .3005722 .094328$

H -3.006294 2.5307201 .979203

C -3.963259 $-0.423905 \quad 0.027314$

C $-6.348839-1.864558-0.325989$

C $-4.454401-1.2515581 .044678$

C $-4.670812-0.340499-1.177742$

C $-5.857381-1.054176-1.349059$

C $-5.640349-1.963263 \quad 0.872044$

H $-3.897716-1.3377421 .971994$

H $-4.305400 \quad 0.272693-1.992704$

H $-6.395743-0.975096-2.289537$

H $-6.007012-2.5985811 .673773$

H $-7.273515-2.418570-0.462883$

F $-1.4882012 .376233-0.104468$

F $-3.6113672 .346974-0.524025$

F -2.226375 $1.327411-1.863637$

SCF Energy $\left(\mathrm{PCM} / \mathrm{mPW} 1 \mathrm{PW} 91 / 6-31+\mathrm{G}^{\star *}\right)=-1524.12133478$

R-MTPA-4 017

B3LYP/6-31G* Geometry

O $-2.4118471 .480150-0.638782$

C -3.6558350 .0644050 .942186$

C -2.067892 $1.776041 \quad 1.758721$

C $-2.870700 \quad 0.7449402 .024713$

C $-1.8852722 .332506 \quad 0.369994$

C -3.683196 $0.914100-0.331038$

C $-4.1010770 .089842-1.516611$

C $-3.982175-1.241219-1.495635$

C $-3.359174-1.968387-0.361934$

O $-3.073486-1.251327 \quad 0.759141$

H $-4.4145991 .723093-0.160881$

H $-4.696033-0.0891481 .263012$

C $-0.4132902 .610809-0.007138$

C $-0.285513 \quad 3.409816-1.297376$

O $-3.056658-3.138436-0.416402$

H $-2.3936813 .316780 \quad 0.329829$

O $0.289914 \quad 1.363532-0.240472$

H -1.514767 $2.269227 \quad 2.552949$

H -2.971619 0.3563603 .034553

H $-4.4641970 .612329-2.398030$

H $-4.255259-1.866169-2.339463$

H 0.0677223 .1258870 .830039

H $-0.7734784 .384402-1.187715$

H $-0.7544262 .871976-2.125220$

H $0.7684003 .578786-1.536476$

C $0.872604 \quad 0.7401970 .798578$

$\begin{array}{lllll}\text { O } & 0.873840 & 1.139257 & 1.938601\end{array}$

C $1.614651-0.5589470 .363136$

C $1.007125-1.193038-0.927855$

O $1.578617-1.492257 \quad 1.408865$

C $0.311040-1.9817071 .864629$

H $-0.116585-2.7115181 .171232$

H $-0.406493-1.1719752 .024309$

H $0.523995-2.4663712 .819686$

C $3.094316-0.2058970 .122607$

C $5.7972010 .414700-0.299850$

C $4.096266-0.949357 \quad 0.754444$

C $3.4557320 .851832-0.722658$

C $4.7997481 .159005-0.931043$

C $5.439944-0.6387910 .541646$

H $3.817007-1.7631171 .411204$

H $2.6900261 .431453-1.225959$

H $5.0652591 .981890-1.589347$

H $6.208682-1.225138 \quad 1.037921$

H $6.8445130 .654968-0.462524$

F $-0.334395-1.327871-0.833491$

F $1.527729-2.420030-1.106612$

F $1.258387-0.479452-2.041432$

SCF Energy $\left(\mathrm{PCM} / \mathrm{mPW} 1 \mathrm{PW} 91 / 6-31+\mathrm{G}^{* *}\right)=-1524.12136062$

R-MTPA-4_018

B3LYP/6-31G* Geometry 
O $2.351508-1.527268-0.723588$

C $3.406930-0.492408 \quad 1.239620$

C $1.453018-1.9985981 .483954$

C $2.278359-1.0986262 .022612$

C $1.568985-2.4347920 .043941$

C $3.592702-1.211480-0.100356$

C $4.395094-0.378512-1.058403$

C $4.5323240 .937868-0.872309$

C $3.816696 \quad 1.667110 \quad 0.202835$

$\begin{array}{lllll}\text { O } & 3.134380 & 0.927111 & 1.116361\end{array}$

H $4.136433-2.1484750 .115215$

H $4.347643-0.583661 \quad 1.801480$

C $0.220283-2.629457-0.670387$

C $0.383687-3.086778-2.114347$

$\begin{array}{lllll}\text { O } & 3.765897 & 2.876311 & 0.254128\end{array}$

H $2.048576-3.4347910 .022981$

O $-0.544292-1.395856-0.722787$

H $\quad 0.660900-2.4526062 .071853$

H $2.166088-0.7794023 .055486$

H $4.835623-0.878016-1.917610$

H $5.0992291 .568948-1.548617$

H $-0.354207-3.356254-0.087684$

H $0.939539-4.030118-2.151341$

H $0.927514-2.336386-2.692264$

H -0.597029 -3.244505 -2.571986

C $-1.477012-1.1883740 .220629$

O $-1.650083-1.8913141 .192294$

C $-2.3616380 .031419-0.161656$

C $-3.3835960 .308508 \quad 0.979415$

O $-3.037370-0.239828-1.375471$

C $-3.854907-1.405884-1.479133$

H $-3.375361-2.291035-1.043004$

H -3.992590 -1.566210 -2.550906

H $-4.829828-1.264464-1.003216$

C -1.527122 $1.295686-0.385727$

C $-0.0687103 .655402-0.761898$

C $-0.418271 \quad 1.5769050 .419874$

C $-1.9109172 .209644-1.372494$

C $-1.180753 \quad 3.383167-1.559526$

C $\quad 0.311740 \quad 2.7500890 .229946$

H -0.1079800 .8789821 .191723$

H -2.772198 $1.994016-1.992896$

$\mathrm{H}-1.4839714 .084493-2.332615$

H 1.1874452 .9451670 .840045

H $0.5055854 .565325-0.913285$

F -4.175753 $-0.751894 \quad 1.247245$

F -4.1936821 .3239460 .624728$

F -2.759797 $0.649105 \quad 2.121317$

SCF Energy $\left(\mathrm{PCM} / \mathrm{mPW} 1 \mathrm{PW} 91 / 6-31+\mathrm{G}^{* *}\right)=-1524.12198060$

\section{R-MTPA-4_019}

B3LYP/6-31G* Geometry

O $-2.411709-1.778859-0.081316$

C $-3.216270 \quad 0.372510-0.958397$

C $-1.360147-0.837032-2.062902$

C $-2.0755110 .280024-1.930052$

C $-1.595291-2.060504-1.213169$

C $-3.567768-1.006846-0.392842$

C $-4.381919-0.8856140 .864239$

C $-4.3746940 .245752 \quad 1.575780$

C -3.5059791 .3983251 .229080$

O -2.8440141 .3493340 .045801$

H -4.156154 -1.529050 - 1.167481

H $-4.1120240 .764223-1.461986$

C $-0.282698-2.675500-0.701723$

C $-0.493160-3.8789530 .206640$

O $-3.3294722 .334796 \quad 1.977277$

H $-2.080702-2.843629-1.829316$

$\begin{array}{llll}\text { O } & 0.434422 & -1.669311 & 0.062547\end{array}$

H $-0.553319-0.897244-2.789768$

H -1.848286 $1.167407-2.513721$

H $-4.944575-1.7566161 .190968$

H -4.9370020 .3615632 .496484$

H $0.328928-2.941191-1.568230$

H -1.055929 -3.5930971 .098098$

H $0.471878-4.2965360 .509361$

H -1.051562 -4.657926 -0.324352
C $1.551009-1.146321-0.470050$

O $2.039748-1.463254-1.532482$

C 2.0985850 .0004200 .422088

C $3.653698 \quad 0.0400850 .311457$

O $1.729741-0.1269421 .770462$

C $2.167710-1.2561162 .524436$

H $1.537806-1.2650183 .416544$

H $3.216547-1.1594932 .822413$

H $2.026935-2.1957871 .980288$

C $1.4567671 .302956-0.099687$

C $0.1955793 .624537-1.040303$

C 0.6314192 .0517800 .745720

C $1.6331051 .715046-1.428946$

C $1.0144952 .877191-1.889008$

$\begin{array}{llll}\text { C } & -0.000461 & 3.202879 & 0.274354\end{array}$

H $\quad 0.4726621 .724323 \quad 1.764520$

H $2.2448161 .130379-2.104966$

H $1.1710163 .193040-2.917313$

H -0.6670573 .7491880 .934695$

H $-0.2943484 .523714-1.404783$

F $4.183006-1.1834510 .561598$

F $4.148108 \quad 0.888015 \quad 1.230927$

F $4.112200 \quad 0.425205-0.889197$

SCF Energy $\left(\mathrm{PCM} / \mathrm{mPW} 1 \mathrm{PW} 91 / 6-31+\mathrm{G}^{* *}\right)=-1524.12237401$

R-MTPA-4_021

B3LYP/6-31 G* Geometry

O $-2.9165740 .593470-0.796658$

C -4.4562800 .6380051 .113417$

C -2.0657240 .0739801 .425106$

C -3.302237 0.1201001 .923866

C -1.7464330 .4692360 .006413$

C $-3.9602101 .328141-0.159874$

C $-5.0731151 .494641-1.154575$

C $-6.173967 \quad 0.740990-1.075230$

C $-6.317778-0.357967-0.087722$

O $-5.351762-0.4748690 .864213$

H $-3.5765392 .318895 \quad 0.137845$

H -5.0223741 .3782151 .697143$

C $-0.858110-0.552148-0.729505$

C $-1.422194-1.963235-0.784305$

O $-7.211617-1.171659-0.139893$

H $-1.197648 \quad 1.4287760 .014954$

O $0.403356-0.535458-0.000812$

H - $1.224654-0.2476672 .034146$

H -3.514052 -0.200187 2.940611

H - $-4.9392382 .222085-1.951241$

H $-6.9938370 .825970-1.780797$

H - $0.673094-0.179626-1.739650$

H -2.409435 -1.939594-1.254029

H $-1.529341-2.3858280 .219553$

H $-0.766613-2.607473-1.376833$

C $1.526589-0.722776-0.712847$

O $1.568476-0.939617-1.902460$

C $2.782674-0.536707 \quad 0.179047$

C $3.948721-1.397697-0.397106$

O $2.545782-0.874334 \quad 1.523204$

C $2.207461-2.2218341 .854413$

H $1.842962-2.1803692 .883116$

H $3.078970-2.8817681 .806365$

H $1.414156-2.6165451 .210282$

C 3.1736750 .9526510 .150205

C 3.9184793 .6550290 .119324

C 3.5305891 .5989561 .338537

C $3.1976471 .666101-1.055857$

C $3.5665363 .010857-1.067543$

C 3.9000192 .9444121 .319704

H 3.5140391 .0472432 .269876

H $2.9312311 .173874-1.984718$

H $3.5798343 .552945-2.009132$

H 4.1755453 .4359052 .248969

H 4.2062664 .7028840 .108177

F $3.565013-2.681283-0.592497$

F $4.968688-1.4121370 .481785$

F $4.415687-0.936846-1.565754$

SCF Energy $\left(\mathrm{PCM} / \mathrm{mPW} 1 \mathrm{PW} 91 / 6-31+\mathrm{G}^{* *}\right)=-1524.12595678$ 
R-MTPA-4 023

B3LYP/6-31 G* Geometry

O $-1.877300 \quad 0.453075-0.336757$

C $-4.2828340 .829325-0.606812$

C $-2.7064572 .723757-0.292657$

C $-3.9602392 .265641-0.303411$

C $-1.5189071 .818785-0.517170$

C $-3.0324740 .079483-1.078121$

C $-3.198347-1.406627-0.949558$

C $-4.154437-1.922467-0.171173$

C $-5.020873-1.0838650 .693647$

$\begin{array}{lllll}0 & -4.904787 & 0.268161 & 0.573828\end{array}$

H $-2.8809400 .341534-2.139892$

H $-5.0295220 .779554-1.412941$

C -0.3542332 .0718690 .453079$

C 0.2462773 .4690140 .379328

O $-5.760081-1.5500011 .530178$

H -1.129869 $1.961285-1.543395$

$\begin{array}{lllll} & 0 & 0.679286 & 1.130084 & 0.056250\end{array}$

H -2.514217 $3.781912-0.135043$

H $-4.8008122 .926509-0.108462$

H -2.492046 -2.037204-1.482698

H $-4.298357-2.991425-0.053175$

H $-0.669124 \quad 1.8243801 .468904$

H $-0.465268 \quad 4.2233200 .728944$

H $0.5497293 .716932-0.643667$

H 1.1312373 .5204581 .019830

C $1.021216 \quad 0.1561120 .916101$

$\begin{array}{lllll}\text { O } & 0.678398 & 0.083745 & 2.071499\end{array}$

C $2.008412-0.8514080 .250321$

C $1.433546-1.347764-1.106336$

O $2.243469-1.9527891 .095472$

C $1.166497-2.8166341 .483532$

H $1.536520-3.3475592 .363226$

H $0.939777-3.5419280 .694777$

H $\quad 0.266973-2.259893 \quad 1.752540$

C $3.372295-0.1476530 .135297$

C $5.882133 \quad 1.1126560 .155205$

C $4.0032620 .169691 \quad 1.347899$

C $4.0113720 .172718-1.066901$

C $5.261140 \quad 0.796784-1.051752$

C $5.246723 \quad 0.796751 \quad 1.357759$

H $3.515071-0.0890322 .281533$

H $3.555323-0.058497-2.020146$

H $5.7479871 .032532-1.994223$

H 5.7204751 .0343862 .306364

H $6.854157 \quad 1.5984860 .160069$

F $0.177598-1.832710-0.937670$

F $2.188714-2.346607-1.596466$

F $1.349974-0.397456-2.058749$

SCF Energy $\left(\mathrm{PCM} / \mathrm{mPW} 1 \mathrm{PW} 91 / 6-31+\mathrm{G}^{* *}\right)=-1524.12005690$

R-MTPA-4_032

B3LYP/6-31G* Geometry

O $-2.969114-0.677430-1.162708$

C -3.464923 $1.554766-0.305594$

C $-1.7290521 .234624-2.033732$

C -2.334834 $2.044534-1.165696$

C -2.079377 $-0.224721-2.178506$

C $-4.0164800 .236381-0.848134$

C $-4.900954-0.4306780 .168650$

C $-4.738381-0.1679761 .468821$

C $-3.636156 \quad 0.6949941 .963221$

$\begin{array}{llll}\text { O } & -2.965542 & 1.445583 & 1.052607\end{array}$

H -4.595192 $0.470143-1.757484$

H $-4.2819802 .289759-0.291342$

C $-0.833747-1.122364-2.121838$

C $-1.159656-2.611311-2.133218$

O $-3.285998 \quad 0.7050753 .123386$

H -2.541419 -0.391917 -3.170955

O $-0.136813-0.745138-0.902984$

H $-0.931508 \quad 1.604808-2.674965$

H -2.029413 $3.081112-1.053887$

H $-5.635333-1.152686-0.179149$

H $-5.328692-0.6443442 .244580$

H $-0.181422-0.873230-2.966068$

H - $-1.774962-2.873990-1.269934$
H $-0.237071-3.194681-2.120760$

H - $1.715894-2.861783-3.043789$

C $1.165452-1.044916-0.820749$

O $1.784757-1.670938-1.653994$

C $1.813717-0.4297490 .452390$

C $2.742521-1.5072521 .092735$

$\begin{array}{llll}\text { O } & 0.877478 & 0.028081 & 1.387912\end{array}$

C $0.015462-0.8856772 .074053$

H $-0.735313-0.2563222 .555230$

H $0.560817-1.4524082 .835488$

H $-0.482119-1.5731221 .384154$

C $2.646326 \quad 0.799706 \quad 0.026528$

C $4.1568273 .054078-0.689988$

C 2.5162491 .9955830 .742490

C $3.5406020 .740387-1.051752$

C $4.2890081 .863064-1.404955$

C 3.2683663 .1147890 .383549

H 1.8245742 .0428991 .573412

H $3.649875-0.177891-1.615928$

H $4.978303 \quad 1.802040-2.242950$

H 3.1569884 .0361400 .949177

H $4.741903 \quad 3.927034-0.967442$

F $2.079795-2.6794061 .255484$

F $3.144853-1.0974832 .308814$

F $3.836895-1.7712230 .363391$

SCF Energy $\left(\mathrm{PCM} / \mathrm{mPW} 1 \mathrm{PW} 91 / 6-31+\mathrm{G}^{* *}\right)=-1524.12343001$

R-MTPA-4 033

B3LYP/6-31G* Geometry

O $2.351225-1.527529-0.723615$

C $3.406690-0.4923651 .239417$

C $1.453010-1.9987621 .484090$

C $2.278375-1.0987392 .022628$

C $1.568801-2.4350240 .044082$

C $3.592409-1.211520-0.100529$

C $4.394665-0.378484-1.058638$

C $4.5321300 .937856-0.872399$

C 3.8166321 .6670980 .202840

$\begin{array}{lllll}\text { O } & 3.133739 & 0.927020 & 1.115904\end{array}$

H $4.136343-2.1484090 .115035$

H $4.347529-0.5833321 .801115$

C $0.220050-2.629791-0.670092$

C $0.383265-3.087437-2.113972$

$\begin{array}{lllll}\text { O } & 3.766291 & 2.876299 & 0.254489\end{array}$

H $2.048447-3.4350010 .023188$

O $-0.544448-1.396122-0.722715$

H $0.661006-2.4528212 .072106$

H $2.166254-0.7795543 .055530$

H $4.834997-0.877939-1.917974$

H $5.0991241 .568894-1.548673$

H $-0.354476-3.356387-0.087163$

H $0.939149-4.030761-2.150840$

H $0.926994-2.337137-2.692097$

H $-0.597512-3.245293-2.571426$

C $-1.476824-1.1881550 .220916$

O $-1.649746-1.890757 \quad 1.192862$

C -2.361399 $0.031559-0.161643$

C -3.3836430 .3086990 .979197$

O $-3.036940-0.239836-1.375539$

C $-3.854389-1.405946-1.479040$

H -3.990534 -1.567671-2.550804

H - $-4.830005-1.263744-1.004735$

H $-3.375699-2.290589-1.040964$

C $-1.5268341 .295764-0.385695$

C $-0.0684423 .655526-0.761867$

C $-1.9105682 .209687-1.372529$

C -0.4180751 .5770780 .420001$

$\begin{array}{llll}\text { C } & 0.311931 & 2.750268 & 0.230052\end{array}$

C $-1.1804153 .383220-1.559564$

H $-2.7717911 .994035-1.993007$

H $-0.1077720 .879218 \quad 1.191911$

H 1.1876072 .9453500 .840200

H -1.483603 $4.084473-2.332732$

H $0.5058314 .565467-0.913213$

F $-4.175741-0.7517831 .246862$

F $-4.193722 \quad 1.3240160 .624149$

F -2.760210 0.6494792 .121204 
SCF Energy $\left(P C M / m P W 1 P W 91 / 6-31+G^{\star \star}\right)=-1524.12198009$

\section{R-MTPA-4 034}

B3LYP/6-31 G* Geometry

O $-2.969017-0.185008-0.926509$

C -4.3435091 .3353590 .421663$

C -1.9899980 .8676601 .034693$

C -3.1754721 .3829241 .365358$

C $-1.7492150 .152380-0.269015$

C $-3.8974710 .896017-0.975430$

C $-5.0696050 .456268-1.805074$

C $-6.2208410 .104242-1.224469$

C -6.3772590 .0196370 .249256$

$\begin{array}{llll}\text { O } & -5.346539 & 0.473249 & 1.014674\end{array}$

H -3.414116 $1.767254-1.449686$

H -4.7939432 .3345360 .332067$

C $-1.003313-1.191075-0.112998$

C $-1.677899-2.1781570 .822980$

O $-7.340367-0.4868370 .777888$

H $-1.1407850 .789328-0.933509$

$\begin{array}{llll}\text { O } & 0.331013 & -0.942153 & 0.423555\end{array}$

H -1.139149 0.9564921 .706162

H -3.3337081 .8673872 .325295$

H -4.939252 $0.408496-2.883221$

H -7.085296 - $0.225341-1.791342$

H $-0.887843-1.610254-1.115240$

H $-1.121746-3.1197310 .843666$

H $-2.693945-2.3717480 .469583$

H -1.736645 -1.784066 1.841983

C $1.289385-0.595485-0.450186$

O $1.096123-0.377980-1.626290$

C $2.704294-0.5610640 .202505$

C $3.500017-1.694699-0.521577$

O $2.705764-0.996117 \quad 1.545751$

C $2.176547-0.1299502 .547878$

H $2.408832-0.6166943 .497695$

H $1.091874-0.0250912 .454157$

H 2.6485030 .8581512 .525352

C 3.4367850 .7791970 .009093

C $4.8964033 .168216-0.246486$

C $3.0283091 .767057-0.894263$

C 4.5942550 .9971160 .773735

C 5.3163512 .1819370 .647961

C $3.7538592 .954224-1.015732$

H $2.1587061 .607259-1.519079$

H 4.9241410 .2317311 .467393

H 6.2089302 .3322401 .249157

H $3.4220833 .710616-1.721884$

H $5.4584154 .092952-0.345329$

F $2.805876-2.851448-0.488739$

F $4.683888-1.912655 \quad 0.073287$

F $3.732392-1.386516-1.805117$

SCF Energy $\left(\mathrm{PCM} / \mathrm{mPW} 1 \mathrm{PW} 91 / 6-31+\mathrm{G}^{* *}\right)=-1524.12297482$

\section{R-MTPA-4_035}

B3LYP/6-31G* Geometry

O $-2.953099-0.2385301 .037081$

C $-4.301673-0.578291-0.984138$

C $-1.839630-0.354924-1.128771$

C $-3.021344-0.392444-1.746788$

C $-1.706910-0.4237540 .370456$

C $-4.018434-0.9926620 .461889$

C $-5.231148-0.8021881 .327396$

C -6.2151030 .0188190 .947728$

C $-6.1239650 .860697-0.271773$

O $-5.0655860 .649567-1.100320$

H $-3.739125-2.0597410 .443878$

H $-4.903367-1.372512-1.449108$

C $-0.7780820 .667461 \quad 0.947480$

C - 1.1848972 .0867110 .592926

O $-6.907811 \quad 1.750955-0.511760$

H - $-1.279072-1.4016390 .654388$

$\begin{array}{lllll}0 & 0.552299 & 0.439319 & 0.394838\end{array}$

H $-0.912590-0.281411-1.690764$

H -3.095056 - $0.310048-2.827945$

H $-5.265936-1.3291152 .277638$

H -7.1017280 .1880921 .549847$
H -0.7299230 .5251012 .028897$

H -2.212974 2.2574500 .922736

H - $-1.1354312 .255368-0.486717$

H -0.5311982 .8092081 .091570$

C $1.372073-0.4040341 .052765$

O $1.126095-0.9054042 .123190$

C $2.670974-0.6993030 .239371$

C $2.215059-1.233335-1.155602$

O $3.327055-1.8176370 .793964$

C $3.994434-1.6547882 .049681$

H $4.778228-0.8913241 .991366$

H $4.452723-2.6253202 .251184$

H $3.288992-1.4102502 .846198$

C 3.6052650 .5084040 .063065

C $5.4316932 .616771-0.282952$

C 3.2524851 .8176900 .406675

C $4.8884840 .266373-0.453269$

C $5.7922011 .312161-0.626604$

C 4.1614312 .8640490 .234537

H 2.2658152 .0354010 .797101

H $5.174891-0.746642-0.712894$

H $6.7801201 .105994-1.029309$

H 3.8699413 .8745650 .507422

H $6.1367303 .432651-0.416548$

F $1.222293-2.141520-1.013729$

F $3.221460-1.832637-1.808964$

F $1.750674-0.246118-1.945009$

SCF Energy $\left(\mathrm{PCM} / \mathrm{mPW} 1 \mathrm{PW} 91 / 6-31+\mathrm{G}^{\star *}\right)=-1524.12386943$

R-MTPA-4_041

B3LYP/6-31G* Geometry

O $-2.952819-0.238366 \quad 1.036863$

C $-4.301208-0.577718-0.984479$

C $-1.839243-0.353353-1.128975$

C $-3.020943-0.390940-1.747027$

C $-1.706561-0.422887 \quad 0.370234$

C $-4.017806-0.9927030 .461328$

C $-5.230642-0.8032901 .326920$

C $-6.215021 \quad 0.0173750 .947625$

C $-6.1242070 .860010-0.271371$

O $-5.0657130 .649860-1.099999$

H -3.737987 -2.059636 0.442770

H $-4.902548-1.371947-1.449887$

C -0.7780630 .6683290 .947793$

C -1.184956 2.0875830 .593509

O

H -1.278472 $-1.400796 \quad 0.653709$

$\begin{array}{lllll}O & 0.552573 & 0.440538 & 0.395395\end{array}$

H $-0.912206-0.279244-1.690891$

H -3.094673 - $0.308056-2.828144$

H $-5.265197-1.3307622 .276869$

H $-7.101766 \quad 0.185871 \quad 1.549785$

H $-0.7301150 .525672 \quad 2.029171$

H -2.213022 $2.258190 \quad 0.923422$

H -1.135597 $2.256489-0.486101$

H -0.5312782 .8100451 .092247$

C $1.371667-0.4040321 .052643$

O $1.124905-0.9066322 .122320$

C $2.670685-0.6993970 .239367$

C $2.214638-1.232850-1.155817$

O $3.326505-1.8180730 .793512$

C $3.993950-1.6559292 .049291$

H $4.451445-2.6268772 .250572$

H $3.288732-1.4109892 .845870$

H $4.778365-0.8930901 .991050$

C 3.6053860 .5080710 .063349

C $5.4324862 .615958-0.282422$

C $4.8885720 .265719-0.452895$

C 3.2529991 .8174520 .407010

$\begin{array}{llll}\text { C } 4.162253 & 2.863561 & 0.234991\end{array}$

C $5.7926181 .311260-0.626103$

H $5.174777-0.747357-0.712518$

H 2.2663822 .0354700 .797407

H 3.8710223 .8741330 .507940

H $6.7805131 .104797-1.028719$

H $6.1377763 .431631-0.415943$

F $1.221787-2.141051-1.014253$ 
F $3.220960-1.832058-1.809384$

F $1.750375-0.245345-1.944868$

SCF Energy $\left(\mathrm{PCM} / \mathrm{mPW} 1 \mathrm{PW} 91 / 6-31+\mathrm{G}^{\star *}\right)=-1524.12386856$

R-MTPA-4 044

B3LYP/6-31G* Geometry

O $1.728398-0.547608-0.412349$

C $4.176734-0.527475-0.561864$

C $2.905167-2.660093-0.524733$

C $4.071139-2.019459-0.419445$

C $1.600710-1.928699-0.733811$

C $2.8480310 .070442-1.037284$

C $2.7762501 .543250-0.755902$

C 3.6022412 .1104850 .128777

C 4.5428151 .3253220 .964440

O $4.647656-0.007810 \quad 0.704496$

H $2.791923-0.097909-2.127036$

H $4.943106-0.279889-1.311052$

C $0.449225-2.4483880 .139970$

C $0.141865-3.932606-0.011348$

O $5.155794 \quad 1.804731 \quad 1.890847$

H $1.285874-2.032723-1.789740$

O $-0.721872-1.705072-0.301468$

H $2.875498-3.745696-0.483781$

H $4.995695-2.562521-0.241725$

H $2.0100332 .118441-1.268610$

H 3.5728213 .1707620 .357378

H $0.644635-2.1896291 .182447$

H $0.961756-4.5465080 .373104$

H $-0.037268-4.198658-1.059044$

H $-0.754529-4.1791950 .565226$

C $-1.397621-0.9945530 .615414$

O $-1.186007-0.999913 \quad 1.806371$

C $-2.469265-0.097715-0.061468$

C $-3.6717190 .079144 \quad 0.916711$

O $-2.916352-0.612962-1.290685$

C -3.601971-1.864432-1.318379

H $-3.619770-2.157321-2.370460$

H -4.629019-1.772218 - 0.951624

H -3.075706 - $2.633821-0.743530$

C $-1.8352541 .275428-0.353706$

C $-0.7388803 .799705-0.891186$

C $-2.0538961 .890301-1.591481$

C $-1.064105 \quad 1.9318130 .614519$

$\begin{array}{llll}C & -0.521057 & 3.187781 & 0.344774\end{array}$

C - $1.5046713 .145330-1.857317$

H -2.650439 $1.383353-2.339249$

H $-0.885911 \quad 1.464803 \quad 1.576347$

H $0.071664 \quad 3.686886 \quad 1.106412$

H - $1.6836083 .613798-2.821560$

H $-0.3199314 .781257-1.097556$

F - $4.118109-1.1173391 .368575$

F $-4.6936890 .669668 \quad 0.268016$

F -3.376222 0.8268231 .988550

SCF Energy $\left(P C M / m P W 1 P W 91 / 6-31+G^{\star *}\right)=-1524.12451788$

R-MTPA-4_048

B3LYP/6-31G* Geometry

O $2.576249-0.946888-1.068511$

C $3.635086-0.279877 \quad 1.047663$

C $1.848895-1.9973330 .993353$

C $2.601974-1.1537611 .699255$

C $1.938813-2.091778-0.510905$

C $3.814745-0.651186-0.428542$

C $4.4793730 .455758-1.194357$

C $4.5072181 .700927-0.710475$

C 3.8039722 .0896190 .538001

$\begin{array}{lllll}\text { O } & 3.236966 & 1.095441 & 1.271688\end{array}$

H $4.454677-1.551124-0.454806$

H $4.607735-0.4107821 .544268$

C $0.567426-2.255867-1.174298$

C $0.607156-2.169306-2.694448$

O 3.6715363 .2429340 .879349

H $2.517400-2.995304-0.789395$

O $-0.295136-1.219156-0.621992$

H $1.118744-2.6336371 .487927$

H $2.489442-1.0634782 .776152$
H $4.9120670 .213030-2.161842$

H $4.9758922 .523749-1.240285$

H $\quad 0.151400-3.219750-0.864512$

H $1.276974-2.939181-3.094015$

H $0.978827-1.192858-3.013269$

H $-0.391754-2.337963-3.103422$

C $-1.610457-1.495275-0.559010$

O $-2.119970-2.483349-1.033491$

C $-2.399625-0.426411 \quad 0.258324$

C $-1.747224-0.3780511 .677312$

O $-3.695376-0.912688 \quad 0.530380$

C $-4.625247-1.005061-0.552953$

H $-5.563296-1.321879-0.091753$

H $-4.308001-1.748865-1.286406$

H $-4.774221-0.034801-1.039949$

C $-2.4152590 .984770-0.352571$

C $-2.5642103 .605050-1.359146$

C $-1.5509141 .405022-1.369162$

C $-3.353207 \quad 1.8960460 .158605$

C -3.426559 $3.194402-0.340681$

C -1.628227 $2.706993-1.868610$

H $-0.7992390 .732548-1.763062$

H -4.0251051 .5790330 .948540$

H $-4.157963 \quad 3.8859210 .068767$

H $-0.947123 \quad 3.016578-2.656537$

H -2.619975 $4.617907-1.748596$

F - $1.517021-1.6326852 .135445$

F -2.549965 $0.232658 \quad 2.562756$

F -0.5766820 .2736291 .666095$

SCF Energy $\left(\mathrm{PCM} / \mathrm{mPW} 1 \mathrm{PW} 91 / 6-31+\mathrm{G}^{\star \star}\right)=-1524.12218167$

R-MTPA-4 050

B3LYP/6-31G* Geometry

O $3.116711-1.059686-0.417161$

C $4.704077-0.176572 \quad 1.235185$

C $2.287700 \quad 0.3458691 .389143$

C $3.536974 \quad 0.591537 \quad 1.787440$

C $1.962916-0.6644830 .318091$

C $4.227503-1.3805160 .418021$

C $5.324364-1.908497-0.461345$

C $6.376474-1.144579-0.770411$

C $6.4748130 .279893-0.363417$

$\begin{array}{lllll}\text { O } & 5.522477 & 0.755268 & 0.485269\end{array}$

H $3.923842-2.1591061 .138867$

H $5.326990-0.5566742 .057842$

C $0.969645-0.141123-0.735470$

C $1.3999731 .144306-1.425684$

O $7.317247 \quad 1.028990-0.802197$

H $1.502829-1.5546530 .786986$

$\begin{array}{lllll}\text { O } & -0.270914 & 0.070204 & 0.000711\end{array}$

H 1.4437390 .8738291 .826228

H 3.7531591 .3507742 .534434

H $5.218391-2.915633-0.856658$

H $7.183140-1.489900-1.408601$

H $0.801464-0.931428-1.471182$

H $2.3828100 .995361-1.881398$

H $1.4695201 .969224-0.710549$

H $0.6839081 .410856-2.206927$

C $-1.413586-0.006308-0.698446$

O $-1.477341-0.282487-1.875366$

C -2.647752 $0.393520 \quad 0.167312$

C $-3.015841 \quad 1.825220-0.336947$

O $-2.303727 \quad 0.6098691 .520691$

C -2.024187 -0.5416732 .314071$

H - $-1.733888-0.1535853 .292859$

H - $1.196627-1.1272701 .899594$

H -2.905251-1.182631 2.424745

C -3.864372 -0.5363970 .012252$

C $-6.164288-2.149616-0.123182$

C $-3.983657-1.520663-0.975818$

C $-4.916624-0.3654540 .927426$

C $-6.055487-1.1647240 .860308$

C $-5.126436-2.321670-1.037332$

H $-3.197819-1.654765-1.707448$

H -4.8359730 .3966861 .694501$

H $-6.858022-1.0159081 .577666$

H $-5.201230-3.081084-1.810985$ 
H $-7.051721-2.774491-0.176860$

F - $-1.9257312 .621601-0.338350$

F - $3.938874 \quad 2.402415 \quad 0.448045$

F -3.497410 $1.784283-1.588126$

SCF Energy $\left(P C M / m P W 1 P W 91 / 6-31+G^{* *}\right)=-1524.12335128$

R-MTPA-4 051

B3LYP/6-31G* Geometry

O $-3.171747 \quad 0.318732 \quad 1.076284$

C $-4.647883-1.027164-0.350811$

C $-2.186400-1.193073-0.564502$

C $-3.396347-1.472759-1.051748$

C $-1.985763-0.3607630 .676073$

C $-4.331481-0.5115181 .055367$

C -5.4757240 .2886051 .609299$

C $-6.399746 \quad 0.807765 \quad 0.795157$

C $-6.2962830 .724647-0.683712$

O $-5.302376-0.049329-1.198330$

H $-4.155454-1.3958451 .691371$

H $-5.341640-1.874018-0.247857$

$\begin{array}{llll}\text { C }-0.924175 & 0.743041 & 0.503146\end{array}$

C $-1.2246921 .745100-0.600811$

O $-7.0100091 .360169-1.425832$

$\mathrm{H}-1.639874-1.0182721 .495025$

$\begin{array}{lllll}\mathrm{O} & 0.302425 & 0.019517 & 0.201532\end{array}$

$\mathrm{H}-1.283708-1.570317-1.037941$

H -3.517553 -2.050810 -1.964025

H -5.5094350 .4517832 .683598$

H $-7.235506 \quad 1.3967321 .158409$

H -0.7942981 .2550321 .459404$

H -2.200120 2.203704 -0.417770

H - $1.2492661 .255619-1.579051$

H $-0.4637492 .530643-0.613387$

C 1.4646400 .5255650 .633435

$\begin{array}{llll}\text { O } & 1.577113 & 1.546226 & 1.275128\end{array}$

C $2.669854-0.4142360 .336374$

C $2.374315-1.392780-0.836451$

O $2.834518-1.280418 \quad 1.451264$

C $3.072426-0.6778372 .726421$

H $3.334855-1.5063373 .387594$

H $2.178248-0.1734633 .109107$

H 3.8963890 .0419202 .689394

C $3.9343910 .381202-0.002977$

C $6.2968351 .716238-0.715883$

C $5.183710-0.1788290 .291696$

C $3.8800471 .607767-0.674098$

C $5.0552792 .270758-1.027969$

C $6.3575560 .488253-0.055161$

H $5.228882-1.1426310 .787445$

H $2.9236952 .058467-0.915763$

H $4.9970073 .224324-1.545354$

H 7.3194940 .0439300 .185614

H $7.2111652 .236613-0.987846$

F $1.387182-2.263791-0.565514$

F $3.465816-2.120228-1.134792$

F $2.029016-0.694443-1.939182$

SCF Energy $\left(P C M / m P W 1 P W 91 / 6-31+G^{\star *}\right)=-1524.12375039$

R-MTPA-4_053

B3LYP/6-31G* Geometry

O $-2.859967-1.010633-0.425681$

C $-4.1023201 .094601-0.637640$

C $-1.6998241 .092384-0.032875$

C $-2.8503421 .759404-0.140497$

C $-1.584749-0.378320-0.340759$

C $-3.791695-0.284778-1.225293$

C $-5.037132-1.115250-1.346571$

C $-6.126939-0.816550-0.632677$

$\begin{array}{llll}\text { C } & -6.141228 & 0.262660 & 0.386608\end{array}$

O $-5.043086 \quad 1.063446 \quad 0.464916$

H -3.357799-0.120003-2.226311

H $-4.5609271 .702142-1.431225$

C $-0.815463-1.172846 \quad 0.736633$

C -1.408107 -1.061143 2.130745

O -7.0480850 .4079631 .174165$

H $-1.046623-0.517043-1.294766$

O $0.549951-0.663795 \quad 0.801488$
H -0.7882561 .5963640 .279223$

H -2.918743 2.8128950 .117968

H $-5.010645-1.976506-2.009328$

H -7.041243 -1.397999-0.689749

H $-0.773957-2.212603 \quad 0.404550$

H - $1.363541-0.0322712 .500321$

H $-0.869789-1.7094572 .828811$

H -2.455862 -1.370740 2.100353

C $1.450488-1.186223-0.058367$

O $1.185063-1.996473-0.914243$

C $2.869504-0.6145860 .225356$

C $3.872237-1.273081-0.766473$

O $3.358538-1.0499461 .482294$

C $2.699417-0.6502142 .684760$

H $1.818813-1.2684512 .882663$

H $2.400267 \quad 0.4020752 .662614$

H $3.435311-0.804198 \quad 3.477874$

$\begin{array}{lllll}\text { C } & 2.918956 & 0.905771 & 0.038049\end{array}$

C $3.1187183 .676078-0.358226$

C $2.1017631 .549319-0.898811$

C 3.8575051 .6579770 .753268

C 3.9503133 .0365270 .561913

C $2.1988652 .927212-1.094051$

H $1.3970390 .975255-1.494146$

H 4.5197831 .1569111 .450916

H 4.6796423 .6090941 .128334

H $1.5586183 .412910-1.825305$

H $3.1920284 .749795-0.507408$

F $3.908039-2.605292-0.637041$

F $5.115392-0.806962-0.538857$

F $3.550655-0.972893-2.038487$

SCF Energy $\left(\mathrm{PCM} / \mathrm{mPW} 1 \mathrm{PW} 91 / 6-31+\mathrm{G}^{* *}\right)=-1524.12283089$

R-MTPA-4_068

B3LYP/6-31G* Geometry

O $-2.130850 \quad 0.256371-0.207060$

C $-4.508948 \quad 0.679819-0.593261$

C $-2.8724482 .541477-0.524593$

C $-4.1433022 .134380-0.509659$

C -1.711283 $1.576269-0.556707$

C $-3.280885-0.166593-0.936430$

C $-3.517821-1.616909-0.628297$

C $-4.482309-1.9825310 .221887$

C $-5.293345-0.9967010 .979702$

$\begin{array}{llll}0 & -5.141611 & 0.317704 & 0.659115\end{array}$

H -3.091380 - $0.043373-2.016033$

H $-5.2573300 .527980-1.384566$

C -0.6209621 .9692630 .462043$

C $0.087808 \quad 3.285521 \quad 0.151083$

O $-6.021582-1.311897 \quad 1.892849$

H $-1.2650491 .561422-1.562222$

$\begin{array}{lllll}\text { O } & 0.349073 & 0.898246 & 0.651190\end{array}$

H -2.641268 $3.603589-0.532690$

H $-4.9612492 .848065-0.457660$

H -2.864925 - $2.348568-1.097708$

H $-4.677980-3.0194310 .474592$

H -1.090869 2.0171341 .447709

H 0.9102343 .4271940 .858455

H $-0.598463 \quad 4.1301220 .266582$

H $0.493823 \quad 3.292486-0.863161$

C $1.0578580 .435166-0.381860$

O $0.995257 \quad 0.833650-1.526100$

C $2.059538-0.6572480 .085885$

C $2.262832-1.681997-1.073247$

O $1.643363-1.3241261 .247981$

C $0.421323-2.0685631 .230969$

H $0.192696-2.2612562 .281306$

H $\quad 0.543792-3.022707 \quad 0.707794$

H $-0.399384-1.4987960 .785702$

C 3.3983160 .0303160 .416546

C 5.8480751 .2472691 .034557

C $4.080107-0.3116481 .589627$

C $3.9534290 .983480-0.448248$

C $5.1710751 .587925-0.137189$

C $5.298608 \quad 0.296016 \quad 1.894397$

H $3.651153-1.0473032 .257913$

H $3.437391 \quad 1.252637-1.363017$ 
H $5.5898922 .326155-0.815758$

H 5.8180540 .0220672 .808814

H 6.7967331 .7196441 .275265

F $1.074392-2.137336-1.541045$

F $2.947909-2.746290-0.616014$

F $2.935359-1.178032-2.118311$

SCF Energy $\left(P C M / m P W 1 P W 91 / 6-31+G^{* *}\right)=-1524.12208930$

R-MTPA-4 069

B3LYP/6-31 G* Geometry

O $-1.9706690 .358216-0.287257$

C $-4.368737 \quad 0.633452-0.705474$

C $-2.8925792 .592640-0.309575$

C $-4.1234292 .083909-0.397334$

C $-1.657927 \quad 1.735551-0.452934$

C $-3.063148-0.069890-1.091755$

C $-3.176976-1.560098-0.953054$

C $-4.158770-2.106394-0.229262$

C $-5.111894-1.2960640 .567523$

$\begin{array}{llll}\text { O } & -5.041240 & 0.059151 & 0.439948\end{array}$

H -2.858478 $0.186506-2.145976$

H -5.061136 $0.548290-1.555992$

C $-0.5630132 .029722 \quad 0.584775$

C -0.0813783 .4739580 .619348$

O $-5.886538-1.782177 \quad 1.359443$

H - $-1.211321 \quad 1.894027-1.453087$

$\begin{array}{lllll}\mathrm{O} & 0.566106 & 1.197231 & 0.189902\end{array}$

H -2.754377 $3.659406-0.154136$

H $-5.0004122 .712201-0.264843$

H $-2.412594-2.166498-1.430623$

H - $4.264436-3.179075-0.105326$

H -0.9020001 .6982421 .567970$

H -0.8721094 .1427450 .972067$

H $0.247887 \quad 3.809317-0.370401$

H $0.760573 \quad 3.5648661 .312057$

C 0.9514370 .2012951 .013190

$\begin{array}{lllll}\text { O } & 0.543620 & 0.038537 & 2.136416\end{array}$

C $2.001480-0.7354910 .334676$

C $1.400054-1.232470-1.017819$

O $2.111623-1.9342131 .070100$

C $2.746213-1.8971242 .352682$

H $2.783968-2.9398172 .675681$

H $2.164539-1.3112503 .066017$

H $3.767445-1.5051092 .285750$

C $3.367482-0.0720390 .082899$

C $5.9252810 .998526-0.389491$

C $3.588764 \quad 1.3068790 .166364$

C $4.447328-0.910073-0.239069$

C $5.713511-0.379039-0.474426$

C $4.8595121 .836967-0.067988$

H $2.772343 \quad 1.9786740 .400447$

H $4.287791-1.980590-0.299452$

H $6.536018-1.044229-0.723003$

H 5.0114332 .9106580 .002773

H $6.9132061 .413037-0.570931$

F $0.146344-1.698054-0.840982$

F $2.131489-2.235501-1.535566$

F $1.354298-0.254295-1.938682$

SCF Energy $(\mathrm{PCM} / \mathrm{mPW}$ PW91/6-31+G** $)=-1524.12125051$

R-MTPA-4_080

B3LYP/6-31 G* Geometry

O $2.365142-0.209430-0.361148$

C $4.806810-0.298872-0.220519$

C $3.408547-2.1951710 .555291$

C $4.599138-1.5939710 .511122$

C $2.170205-1.591875-0.063711$

C $3.5779280 .045046-1.065650$

C $3.5857861 .491394-1.469297$

C $4.3253132 .380934-0.799879$

C 5.0877782 .0253720 .423396

$\begin{array}{lllll}\text { O } & 5.145735 & 0.708299 & 0.763817\end{array}$

H $3.614657-0.590267-1.967022$

H $5.669066-0.387356-0.897327$

C $0.939464-1.6596200 .851012$

C $0.586718-3.0548721 .355642$

O $5.5950762 .854969 \quad 1.142655$
H $1.921117-2.134054-0.994812$

O $-0.159456-1.177305 \quad 0.027944$

H $3.307687-3.1663361 .031739$

H $5.468710-2.0366350 .989590$

H $2.9549521 .789476-2.302995$

H $4.3507133 .434351-1.058642$

H $1.072373-0.9658391 .684036$

H $1.341829-3.4248632 .055331$

H $0.490571-3.7640090 .526264$

H $-0.366252-3.0145231 .889467$

C $-1.149973-0.516948 \quad 0.649590$

O $-1.181423-0.292673 \quad 1.837057$

C $-2.224513-0.058150-0.392984$

C $-2.850130-1.329872-1.038239$

O $-1.6074230 .602946-1.479298$

C $-0.709987 \quad 1.662841-1.139803$

H - $0.4991712 .179207-2.079251$

H $0.2251661 .268235-0.727205$

H - $-1.1638472 .370085-0.435694$

C $-3.353746 \quad 0.7716720 .235849$

C -5.5130132 .2656721 .236065$

C $-3.8921901 .832168-0.504079$

C -3.9303990 .4463781 .471290$

C $-4.999724 \quad 1.1932341 .966024$

C $-4.9576172 .580196-0.004243$

H -3.478696 $2.059418-1.480209$

H -3.544374 -0.3800732 .052651$

H $-5.431267 \quad 0.9322882 .928571$

H $-5.357303 \quad 3.403430-0.590360$

H $-6.3437772 .846597 \quad 1.627742$

F $-1.982632-2.015698-1.794748$

F $-3.897750-1.011399-1.814892$

F $-3.289811-2.160340-0.061622$

SCF Energy $\left(\mathrm{PCM} / \mathrm{mPW} 1 \mathrm{PW} 91 / 6-31+\mathrm{G}^{* *}\right)=-1524.12307498$

R-MTPA-4_098

B3LYP/6-31G* Geometry

O $2.998432-0.630313-0.898124$

C $4.316539-0.2686731 .141526$

C $1.842610-0.1932361 .204763$

C 3.0077630 .0333731 .813462

C $1.752637-0.700378-0.211767$

C $4.100230-1.102152-0.124265$

C $5.318263-1.077452-1.002787$

C $6.231798-0.110705-0.871555$

C $6.0513351 .036423 \quad 0.053537$

$\begin{array}{llll}O & 4.988780 & 0.993681 & 0.902889\end{array}$

H $3.897879-2.1367610 .201255$

H $4.959962-0.8494391 .818161$

C $0.7620910 .101134-1.082402$

C $1.0977491 .576061-1.228652$

O $6.7648802 .013294 \quad 0.031463$

H $1.395691-1.746943-0.193529$

O $-0.526322-0.033792-0.415909$

H $0.897130-0.0325751 .716112$

H 3.0484340 .4270812 .825628

H $5.414825-1.850711-1.760807$

H $7.119353-0.056008-1.493302$

H $\quad 0.691138-0.378403-2.060264$

H $2.092197 \quad 1.678621-1.670987$

H $1.1000082 .081187-0.257784$

H $\quad 0.3690842 .066262-1.881397$

C -1.569857-0.530552-1.106898

O $-1.552087-0.840711-2.272304$

C $-2.840766-0.648601-0.212676$

C $-2.462791-1.1383821 .221008$

O $-3.744463-1.550522-0.792895$

C $-3.369269-2.917493-0.978580$

H $-4.129837-3.330379-1.644160$

H -3.379961 $-3.468974-0.032656$

H -2.390587 -3.010737-1.456856

C -3.555159 $0.715741-0.143167$

C $-4.901756 \quad 3.176395-0.027054$

C $-4.9201590 .791252-0.443910$

C $-2.869623 \quad 1.8844480 .217363$

C $-3.541363 \quad 3.1052980 .273801$

C $-5.5867012 .015398-0.384862$ 
H $-5.451894-0.107856-0.726341$

$\begin{array}{llll}\mathrm{H} & -1.814351 & 1.846981 & 0.457948\end{array}$

H -2.995518 4.0021680 .553744

H $-6.6464612 .057636-0.621470$

H -5.4227584 .1290320 .015984$

F - $-1.612239-2.1963291 .164053$

F $-3.564747-1.5412151 .873367$

F - $1.864072-0.1980851 .976853$

SCF Energy $\left(\mathrm{PCM} / \mathrm{mPW} 1 \mathrm{PW} 91 / 6-31+\mathrm{G}^{* *}\right)=-1524.12441753$

R-MTPA-4 110

B3LYP/6-31 G* Geometry

O $-2.230841-0.3067320 .520643$

C $-4.676642-0.231140 \quad 0.386580$

C $-3.428397-2.295862-0.186368$

C $-4.572446-1.609298-0.200595$

C $-2.140002-1.721240 \quad 0.350901$

C -3.4190570 .1084861 .190129$

C $-3.315377 \quad 1.5867501 .433611$

C -3.989673 2.4516330 .669919

C -4.786153 $2.024152-0.507559$

$\begin{array}{llll}\text { O } & -4.948861 & 0.686059 & -0.700732\end{array}$

H -3.498414 -0.4217232 .154619$

H -5.536889 -0.1813591 .069849$

C $-0.947002-1.957920-0.586028$

C $-0.657133-3.421296-0.902432$

O $-5.2336462 .806166-1.314418$

H -1.903003 -2.191562 1.323573

O $0.197247-1.4058160 .121554$

H -3.402436 - $3.317044-0.556725$

H -5.477706 -2.037161 - 0.623033

H -2.6560311 .9266192 .228363$

H -3.9310873 .5258450 .810759$

H - $1.091853-1.381938-1.502832$

H -1.450651-3.858312 -1.515768

H $-0.549178-4.0111850 .014259$

H $\quad 0.275267-3.488985-1.468527$

C $1.167134-0.860595-0.624691$

O $1.165330-0.826215-1.835591$

C $2.348304-0.3471420 .252816$

C $3.494088-1.374891-0.007017$

O $2.095938-0.4936421 .634276$

C $1.150901 \quad 0.4006442 .224657$

H 0.1687590 .3239961 .748016

H 1.5014731 .4380642 .187822

H 1.0740760 .0838643 .267439

C $2.8301601 .069606-0.108469$

C $3.8046133 .659399-0.609314$

C $2.4189551 .774720-1.245340$

C 3.7398541 .6771160 .772924

C 4.2229452 .9597520 .524183

C $2.9032333 .062194-1.488728$

H $1.7340491 .320625-1.949470$

H 4.0635101 .1390551 .656938

H 4.9267173 .4117231 .218029

H $2.5715533 .595140-2.375703$

H $4.1795894 .660534-0.804467$

F $3.044147-2.6379130 .162663$

F $4.521372-1.1955380 .838578$

F $3.962380-1.271567-1.259470$

SCF Energy $\left(P C M / m P W 1 P W 91 / 6-31+G^{* *}\right)=-1524.12357920$

R-MTPA-4_111

B3LYP/6-31 G* Geometry

$\begin{array}{lllll}\text { O } & 1.929746 & 0.430276 & 0.305556\end{array}$

C $4.3508380 .721304 \quad 0.520875$

C 2.8428722 .6646200 .172955

C 4.0782132 .1588630 .176880

C $1.6259401 .813608 \quad 0.446964$

C $3.0822970 .038178 \quad 1.041541$

C $3.188903-1.4568730 .963673$

C $4.104541-2.0360510 .181060$

C $4.980828-1.261125-0.732329$

O $4.9227150 .097483-0.653730$

H $2.959900 \quad 0.3430572 .095449$

H $5.1127920 .667476 \quad 1.312258$

C $0.4548822 .081249-0.511489$
C $-0.0831253 .505143-0.480287$

O $5.678802-1.783899-1.570675$

H 1.2618192 .0043681 .474563

O $-0.6115591 .196426-0.066845$

H $2.6883573 .723987-0.014829$

H $4.9396032 .780293-0.053564$

H $2.475162-2.0405841 .539016$

H $4.205992-3.1131600 .098056$

H $0.7401181 .783999-1.522428$

H $0.6504344 .212082-0.879797$

H $-0.345383 \quad 3.8102720 .538959$

H $-0.9814153 .570099-1.100841$

C $-0.9871570 .194243-0.886463$

O $-0.6280600 .054138-2.028511$

C $-1.995544-0.774688-0.197771$

C $-1.598966-1.0504941 .286289$

O $-2.034873-1.985720-0.903376$

C $-0.853762-2.785740-1.011867$

H $-1.057233-3.481874-1.828049$

H $-0.673052-3.351050-0.091252$

H $0.025989-2.188422-1.263551$

C $-3.413354-0.172075-0.266636$

C $-6.0109180 .884154-0.415373$

C $-4.453454-0.924138-0.824764$

C -3.6855311 .1159520 .216058$

C -4.9768561 .6372660 .141520$

C $-5.743207-0.396839-0.897260$

H $-4.246057-1.917084-1.201559$

H -2.894232 1.7116830 .653958

H -5.171822 2.6368690 .520750

H $-6.539719-0.993505-1.333729$

H $-7.0160141 .293193-0.473953$

F $-0.285400-1.3766671 .391967$

F -2.308519-2.089983 1.759812

F -1.809864 -0.006758 2.107080

SCF Energy $\left(\mathrm{PCM} / \mathrm{mPW} 1 \mathrm{PW} 91 / 6-31+\mathrm{G}^{* *}\right)=-1524.12285749$

S-MPTA-3_001

B3LYP/6-31G* Geometry

O -1.837675 $-0.240656-0.146809$

C $-4.144235-0.382324-0.966291$

C $-2.568319-2.275833-1.246241$

C $-3.820832-1.816401-1.280516$

C $-1.403486-1.411996-0.829903$

C $-2.8644570 .451469-0.846990$

C $-3.1167661 .743651-0.125752$

C $-4.203742 \quad 1.8981200 .636424$

C $-5.152470 \quad 0.789510 \quad 0.907464$

$\begin{array}{llll}0 & -4.968086 & -0.374642 & 0.225756\end{array}$

H -2.522598 $0.664676-1.874934$

H $-4.7526870 .048041-1.775056$

C $-0.436574-2.1602360 .104064$

C $-1.019945-2.5040871 .465132$

$\begin{array}{lllll}\text { O } & -6.023477 & 0.862971 & 1.744297\end{array}$

H $-0.832365-1.121880-1.728761$

O $0.720600-1.3159960 .357269$

H $-2.346163-3.301044-1.536852$

H -4.651592 - $2.461057-1.555220$

H -2.366911 $2.525191-0.219320$

H -4.4132032 .8143311 .178661$

H $-0.088915-3.054544-0.422475$

H -1.881906 -3.1681581 .347595$

H -1.349422 -1.5982661 .978655$

H $-0.268593-3.0100172 .078050$

C $1.664894-1.261152-0.592989$

O $1.643584-1.867056-1.636171$

C $2.778956-0.235398-0.206769$

C $3.435225-0.6579991 .137384$

O $3.726511-0.376883-1.241166$

C $4.8614520 .491478-1.264346$

H $4.5897471 .530120-1.047033$

H $5.2510910 .425571-2.282656$

H $5.6335140 .163768-0.561122$

C $2.1355021 .160673-0.188718$

C $0.8972463 .676071-0.358836$

C $1.7788171 .698816-1.434651$

C 1.8591461 .8923570 .971572 
C 1.2463293 .1439520 .882442

C $1.1648152 .946885-1.519883$

H $1.9953411 .131052-2.334701$

H $2.110251 \quad 1.4986561 .948254$

H 1.0444513 .7032141 .791885

H $0.9013743 .351544-2.493553$

H $0.4276474 .654199-0.422254$

F $4.391840 \quad 0.225866 \quad 1.507479$

F $2.572881-0.7464422 .168471$

F $4.021940-1.8597190 .996257$

SCF Energy $\left(\mathrm{PCM} / \mathrm{mPW} 1 \mathrm{PW} 91 / 6-31+\mathrm{G}^{\star \star}\right)=-1524.12269203$

S-MPTA-3 005

B3LYP/6-31G* Geometry

O $2.904825-1.202142-0.435273$

C $4.0059290 .968148-0.751211$

C $1.535598 \quad 0.794680-0.645228$

C $2.6304361 .556743-0.623339$

C $1.598435-0.710336-0.722887$

C $3.930501-0.509697-1.143880$

C $5.229673-1.211968-0.871932$

C $6.121527-0.691688-0.023496$

C 5.8469820 .5310750 .772351

$\begin{array}{lllll}\text { O } & 4.701198 & 1.211540 & 0.497577\end{array}$

H $3.709811-0.545430-2.224586$

H $4.5690631 .494700-1.535452$

C $0.669512-1.390755 \quad 0.299146$

C $0.733064-2.910277 \quad 0.261338$

O $\quad 6.5614320 .8958701 .678114$

H $1.289555-1.041515-1.732335$

O $-0.669525-0.945296-0.066054$

H $0.5449311 .239081-0.604235$

H $2.5624232 .636878-0.524333$

H $5.405185-2.164415-1.365919$

H $7.066675-1.1750890 .200848$

H $\quad 0.893398-1.0175831 .301319$

H $1.750627-3.2414840 .482376$

H $0.447701-3.283531-0.728421$

H $\quad 0.054964-3.3322601 .007999$

C - $1.584088-0.8557130 .913630$

O $-1.387276-1.1239582 .075230$

C $-2.933810-0.2858880 .377095$

C $-3.357192-1.082635-0.889198$

O $-3.935521-0.365621 \quad 1.362502$

C $-4.362273-1.6437531 .851803$

H $-5.014384-2.1489171 .131319$

H $-4.932419-1.4198822 .755666$

H $-3.518232-2.2870712 .110742$

C -2.7455021 .2201500 .131386$

C -2.432227 $4.002010-0.098178$

C -2.2587831 .9762651 .208686$

C $-3.0896131 .874851-1.056528$

C $-2.9304953 .258111-1.166684$

C $-2.098761 \quad 3.3555181 .093538$

H -2.0173981 .4794852 .143169$

H -3.486984 1.326096-1.900302

H -3.204161 $3.752007-2.095028$

H -1.718308 3.9238361 .937749

H -2.310194 $5.077808-0.190233$

F -4.635868 - $0.814433-1.212533$

F -2.600923 -0.826838-1.973172

F $-3.268410-2.414830-0.654916$

SCF Energy $\left(\mathrm{PCM} / \mathrm{mPW} 1 \mathrm{PW} 91 / 6-31+\mathrm{G}^{* *}\right)=-1524.12246037$

S-MPTA-3_009

B3LYP/6-31G* Geometry

O $2.939951-1.224625-0.345952$

C $4.0057350 .944564-0.771928$

C $1.5387720 .736846-0.649095$

C $2.6213761 .516437-0.666899$

C $1.625263-0.769008-0.654996$

C $3.952294-0.552034-1.091000$

C $5.263145-1.220027-0.790217$

C $6.150825-0.6441090 .026044$

C 5.8619580 .6136810 .759653

$\begin{array}{lllll}\text { O } & 4.703534 & 1.260841 & 0.459045\end{array}$

H $3.729050-0.643765-2.167987$
H $4.5566161 .440549-1.584389$

C $0.705993-1.4172180 .396616$

C $0.795354-2.935898 \quad 0.425883$

O 6.5767401 .0362521 .639789

H $1.322792-1.151883-1.648053$

O $-0.637078-1.0060470 .012215$

H $0.5412361 .166902-0.629274$

H $2.5363732 .598927-0.619815$

H $5.450522-2.193965-1.235544$

H $7.104059-1.1014620 .269897$

H $0.924520-0.9974511 .381672$

H $1.818653-3.2398230 .659369$

H $0.516042-3.356558-0.546560$

H $0.124782-3.3355131 .191092$

C $-1.559211-0.9279620 .988502$

O $-1.366862-1.1807212 .149777$

C -2.911848 -0.3791340 .430720$

C $-3.393342-1.275197-0.743051$

O $-3.799665-0.538327 \quad 1.515042$

C $-5.1127380 .019284 \quad 1.427053$

H -5.1040821 .0230950 .989166$

H $-5.4677370 .079007 \quad 2.458296$

H $-5.785162-0.6227130 .849847$

C $-2.693337 \quad 1.096558 \quad 0.062014$

C $-2.2365743 .829517-0.401818$

C $-2.7467801 .611834-1.238615$

C $-2.407719 \quad 1.965467 \quad 1.127409$

C $-2.181053 \quad 3.3206290 .897759$

C -2.520188 $2.972040-1.464229$

H -2.962021 $0.968441-2.082267$

H -2.368876 1.5674372 .137120

H - 1.9602853 .9778711 .734282

H -2.569990 $3.358335-2.478639$

H $-2.0614564 .886334-0.583580$

F - $-4.590035-0.850925-1.214804$

F $-2.553338-1.302026-1.797184$

F - $3.548354-2.540421-0.316101$

SCF Energy $\left(P C M / m P W 1 P W 91 / 6-31+G^{\star \star}\right)=-1524.12294161$

S-MPTA-3_010

B3LYP/6-31G* Geometry

O -2.309537 -1.419910 -0.929878

C $-3.544098-0.570501 \quad 1.005130$

C -1.633495 -2.122643 1.291478

C $-2.496181-1.2598181 .831376$

C $-1.610111-2.418019-0.188849$

C $-3.608500-1.169176-0.401541$

C $-4.335007-0.258834-1.349292$

C $-4.4922821 .036949-1.062649$

C -3.8515561 .6680670 .116430$

$\begin{array}{llll}\text { O } & -3.243845 & 0.849370 & 1.016654\end{array}$

H - $-4.156469-2.124678-0.320688$

H $-4.532485-0.6887531 .471965$

C $-0.200766-2.509833-0.784254$

C $0.515094-3.819917-0.469784$

$\begin{array}{lllll} & \text { O } & -3.786411 & 2.869389 & 0.259841\end{array}$

H -2.091112 -3.398031 - 0.373790

O $0.575491-1.408768-0.240677$

H $-0.905282-2.635698 \quad 1.914384$

H -2.470771-1.027343 2.892526

H $-4.704361-0.683351-2.279326$

H $-5.0064001 .726402-1.723896$

H $-0.282152-2.369066-1.864276$

H $-0.057339-4.671018-0.854229$

H $0.658594-3.9500910 .607808$

H $1.497056-3.827207-0.950001$

C $1.555674-0.902802-1.014269$

O $1.774336-1.239032-2.153145$

C $2.4042630 .164834-0.262884$

C $2.648307-0.2781091 .214242$

O $3.6350320 .323423-0.921216$

C $4.548020-0.771316-1.006396$

H $5.038365-0.963468-0.046266$

H $5.297333-0.456556-1.735713$

H $4.063141-1.682255-1.368427$

C $1.7116151 .541056-0.279086$

C $0.5369024 .086446-0.230720$ 
C $\quad 0.353570 \quad 1.688096 \quad 0.035546$

C $2.4753042 .678500-0.569208$

C $1.8875063 .942945-0.548015$

C -0.2280532 .9569060 .061796$

H -0.2591920 .8236020 .262536$

H $3.5239242 .566881-0.812429$

H $2.4921164 .816598-0.777510$

H -1.2790803 .0603310 .314446$

H $0.0803495 .072531-0.210674$

F $3.6382990 .454231 \quad 1.753429$

F $1.566639-0.1475601 .997738$

F $3.030174-1.5824051 .278458$

SCF Energy $\left(\mathrm{PCM} / \mathrm{mPW} 1 \mathrm{PW} 91 / 6-31+\mathrm{G}^{* *}\right)=-1524.12411902$

S-MPTA-3_014

B3LYP/6-31G* Geometry

O $3.259623-0.855245-0.864669$

C $4.5056931 .076107-0.005125$

C $2.0452111 .170838-0.289205$

C 3.1598501 .7042350 .213818

C $2.041877-0.135546-1.043369$

C $4.418886-0.045004-1.044231$

C $5.620882-0.942686-0.977732$

C $6.390627-0.971078 \quad 0.114321$

C $6.053845-0.2166801 .347986$

O $5.0032290 .645104 \quad 1.286803$

H $4.3722050 .438063-2.035478$

H $5.2139641 .828181-0.382327$

C $0.930556-1.090018-0.568120$

C $0.910145-2.414586-1.316677$

O $6.631913-0.3884792 .397066$

H $1.8810650 .061773-2.120053$

O $-0.308892-0.364020-0.809468$

H $1.0863651 .667191-0.174932$

H 3.1325442 .6260050 .788974

H $5.824574-1.583129-1.832352$

H $7.262134-1.6118480 .199448$

H $1.029302-1.2554690 .507726$

H $1.866277-2.926571-1.182988$

H $\quad 0.745103-2.248887-2.387117$

H $0.111386-3.051866-0.928536$

C -1.354968 -0.667672 -0.026594

O $-1.358130-1.5066290 .840282$

C -2.586479 $0.236677-0.363293$

C -2.2431331 .6719340 .142841$

O $-2.6733450 .233179-1.774541$

C -3.638560 $1.095552-2.384889$

$\mathrm{H}-4.6288740 .989437-1.929335$

H $-3.6839230 .781796-3.429838$

H -3.324098 2.143461-2.338068

C $-3.835971-0.3449300 .309954$

C $-6.090241-1.5540531 .469463$

C $-4.576566-1.299526-0.398059$

C $-4.225316-0.0160871 .613682$

C $-5.347986-0.6149082 .185439$

C $-5.697776-1.8976430 .175501$

H $-4.266204-1.574648-1.400730$

H -3.6571650 .6987452 .196614$

H $-5.638087-0.3462433 .197462$

H $-6.261581-2.634687-0.389953$

H $-6.964113-2.0182551 .918401$

F -3.331068 2.4649020 .204405

F $-1.690657 \quad 1.639651 \quad 1.378073$

F - $1.3582192 .277239-0.673030$

SCF Energy $\left(\mathrm{PCM} / \mathrm{mPW} 1 \mathrm{PW} 91 / 6-31+\mathrm{G}^{\star \star}\right)=-1524.12127851$

S-MPTA-3_015

B3LYP/6-31 G* Geometry

O $2.6230391 .042655-1.013950$

C 3.8932000 .7157651 .059373

C 2.0375902 .3534540 .944696

C $2.9003931 .653983 \quad 1.682881$

C $1.9595282 .220286-0.556157$

C $3.9284620 .892680-0.460869$

C $4.582601-0.282211-1.129862$

C $4.685678-1.455504-0.497929$

C 4.065571-1.699444 0.827921
O $3.544663-0.6267671 .484218$

H $4.514351 \quad 1.805775-0.665268$

H $4.9018880 .918978 \quad 1.446816$

C $0.5316532 .154746-1.110643$

C $-0.2457113 .462001-1.002750$

O $3.944449-2.806398 \quad 1.302017$

H $2.433598 \quad 3.104117-1.025281$

O $-0.160498 \quad 1.116550-0.372271$

H 1.3551423 .0552891 .417148

H 2.9139961 .7403032 .766038

H $4.939814-0.153324-2.148415$

H $5.141995-2.328139-0.953334$

H $0.5952201 .841320-2.155526$

H $0.279276 \quad 4.265221-1.531555$

H -0.3885803 .7625190 .039928$

H -1.230324 $3.340096-1.461751$

C $-1.0526660 .379209-1.053168$

O $-1.3440860 .543852-2.217082$

C $-1.724381-0.668387-0.126954$

C $-2.154754-1.898133-0.986231$

O $-0.892413-1.0782840 .924475$

C $0.350788-1.7256050 .625103$

H $0.927752-1.169539-0.120407$

H $\quad 0.194613-2.754417 \quad 0.287727$

H $0.906943-1.7307421 .563191$

C -2.967111 $-0.015040 \quad 0.507544$

C $-5.233027 \quad 1.1429701 .684818$

C $-3.8688970 .724272-0.269857$

C $-3.209226-0.1733741 .876181$

C -4.3377270 .4045582 .459065$

C $-4.994326 \quad 1.3003120 .318823$

H -3.694931 $0.847412-1.333187$

H -2.511578 -0.7447922 .475122$

H -4.5155680 .2739543 .523250$

H $-5.6857231 .871348-0.295031$

H -6.1108301 .5921262 .141847$

F $-2.547140-2.896247-0.173310$

F $-3.167835-1.628753-1.825018$

F - $1.126593-2.362733-1.733060$

SCF Energy $\left(P C M / m P W 1 P W 91 / 6-31+G^{* *}\right)=-1524.12577152$

S-MPTA-3_024

B3LYP/6-31G* Geometry

O -1.994266 -0.084787 -0.259518

C $-4.334509-0.340395-0.942214$

C $-2.777901-2.265170-0.999473$

C $-4.029176-1.806588-1.061361$

C $-1.593685-1.367044-0.741621$

C $-3.0515780 .491096-1.024639$

C $-3.2798581 .886704-0.519477$

C -4.3175612 .1611590 .276778$

C $-5.2305281 .107806 \quad 0.788051$

$\begin{array}{lllll}\text { O } & -5.078101 & -0.148846 & 0.286425\end{array}$

H -2.754520 $0.524374-2.086679$

H $-4.991566-0.027461-1.766544$

C $-0.643788-1.9833170 .301298$

C -1.232940 -2.093249 1.698534

O $-6.0432501 .312528 \quad 1.660091$

H - $-1.023844-1.244420-1.677445$

O $\quad 0.549743-1.158286 \quad 0.432613$

H -2.567444 -3.322851-1.146447

H - $-4.869551-2.477627-1.217429$

H -2.562563 $2.653338-0.802245$

H -4.5095733 .1552430 .666860$

H $-0.326214-2.959027-0.079614$

H -2.112894 -2.743639 1.686010

H - $1.536249-1.1098642 .064505$

H $-0.492551-2.5159372 .383188$

C $1.493320-1.269907-0.516268$

O $1.393394-1.968535-1.499070$

C $2.757806-0.419203-0.187368$

C $3.276644-0.8879861 .208924$

O $3.811155-0.793542-1.047401$

C $3.727751-0.423183-2.426594$

H $2.893695-0.922703-2.923164$

H $4.669796-0.760026-2.864781$

H $3.6433580 .662707-2.547892$ 
C $2.5212351 .101510-0.166872$

C $2.2669973 .901003-0.115741$

C $1.2555271 .696659-0.105445$

C $3.6577931 .925603-0.199110$

C $3.5318243 .312613-0.174232$

C $1.1353213 .088412-0.080880$

H $\quad 0.3576371 .092217-0.057900$

H $4.6407971 .470244-0.244034$

H $4.4235753 .933074-0.198842$

H $0.1458873 .535376-0.026485$

H $2.1666214 .982844-0.094900$

F $4.524267-0.4459121 .437058$

F $2.497091-0.4393962 .206038$

F $3.300869-2.236167 \quad 1.276347$

SCF Energy $\left(P C M / m P W 1 P W 91 / 6-31+G^{\star \star}\right)=-1524.12451170$

S-MPTA-3 025

B3LYP/6-31G* Geometry

O $2.1806170 .209921-0.100167$

C $4.412776 \quad 1.1140150 .372808$

C 2.6586601 .0647842 .121238

C $3.941677 \quad 1.2285321 .794919$

C 1.6026800 .6934961 .107364

C $3.2238521 .044915-0.591263$

C $3.6425690 .514251-1.932121$

C $4.779900-0.174103-2.069326$

C $5.631508-0.547201-0.912743$

$\begin{array}{llll}\text { O } & 5.298847 & -0.029791 & 0.302179\end{array}$

H $2.8422692 .074676-0.704686$

H 5.0111151 .9972090 .105426

C $0.659756-0.3865931 .651110$

C $1.281866-1.7716971 .764379$

O $6.551183-1.328248-1.000809$

H $\quad 0.982804 \quad 1.5818280 .884439$

O $-0.470866-0.4285780 .734154$

H 2.3273931 .2049913 .148610

H 4.6900211 .4643212 .546939

H $2.9761730 .683378-2.774082$

H $5.107685-0.578278-3.021397$

H $0.278782-0.0573992 .622557$

H $2.160365-1.7378522 .417154$

H $1.596205-2.1287600 .781407$

H $\quad 0.557773-2.4697752 .192765$

C $-1.667948-0.7306351 .261842$

O $-1.880698-0.9576032 .426620$

C $-2.786950-0.6694890 .173079$

C $-2.421404-1.618350-1.000930$

O $-3.917803-1.187961 \quad 0.839152$

C $-5.191978-1.134727 \quad 0.194497$

H $-5.357663-0.176915-0.310181$

H $-5.923973-1.2500420 .997167$

H $-5.314257-1.950114-0.525139$

C $-2.9666840 .803216-0.226164$

C $-3.3529013 .535514-0.751131$

C -3.4645651 .6595320 .768620$

C $-2.6616831 .331138-1.485792$

C $-2.8574922 .690092-1.743008$

C $-3.6560643 .014650 \quad 0.508812$

H -3.7045901 .2491941 .745171$

H -2.270488 $0.697957-2.271765$

H -2.622032 $3.084458-2.727869$

H $-4.0433083 .662724 \quad 1.290394$

H -3.503821 $4.591624-0.957597$

F -3.401380 -1.625570 -1.936873

F - $1.282567-1.291702-1.640523$

F - $-2.285622-2.875304-0.543212$

SCF Energy $\left(\mathrm{PCM} / \mathrm{mPW} 1 \mathrm{PW} 91 / 6-31+\mathrm{G}^{* *}\right)=-1524.12270006$

S-MPTA-3_026

B3LYP/6-31G* Geometry

O -2.1711960 .2519950 .115408$

C $-4.3908631 .162628-0.397491$

C $-2.6014041 .120508-2.110487$

C $-3.8904061 .285331-1.809057$

C -1.567639 $0.740455-1.077050$

C -3.2221201 .0873190 .590164$

C -3.6684800 .5503361 .919555$
C -4.807947-0.139525 2.029034

C $-5.634021-0.5077490 .852594$

$\begin{array}{lllll} & -5.277485 & 0.018121 & -0.352112\end{array}$

H -2.840473 2.1153980 .716327

H $-4.9945662 .044288-0.137454$

C $-0.617823-0.341106-1.605390$

C $-1.243410-1.722600-1.739258$

O $-6.553160-1.2917400 .916149$

H $-0.9490191 .625202-0.837225$

O $0.491058-0.392252-0.660090$

H -2.248854 $1.267226-3.129852$

H $-4.622547 \quad 1.528211-2.574641$

H -3.0201660 .7161232 .776131$

H $-5.155639-0.5486602 .971877$

H $-0.211036-0.008777-2.565169$

H -2.107442 -1.680489-2.410519

H - $1.580758-2.083169-0.765304$

H $-0.514192-2.422284-2.156125$

C $1.699731-0.701339-1.149688$

O $1.941375-0.940649-2.311103$

C $2.797368-0.639195-0.042642$

C $2.321415-1.4280591 .209007$

O $4.013126-1.168319-0.516982$

C $4.094895-2.544298-0.908592$

H $4.149160-3.204281-0.036420$

H $5.026333-2.619157-1.473643$

H $3.261653-2.838880-1.550772$

$\begin{array}{llll}\text { C } 3.099261 & 0.844431 & 0.228685\end{array}$

C 3.7779003 .5529640 .532449

C $3.5234401 .603375-0.872677$

C 3.0306411 .4533091 .486300

C 3.3689132 .8007261 .632219

C $3.8561882 .947508-0.723288$

H $3.601601 \quad 1.128903-1.845631$

H 2.7222540 .8950322 .360247

H 3.3144553 .2581412 .616412

H $4.1821123 .519468-1.587826$

H 4.0395334 .6007850 .652901

F $3.339533-1.5980532 .073254$

F $1.314081-0.8384451 .877482$

F $1.884611-2.6591040 .847066$

SCF Energy $\left(\mathrm{PCM} / \mathrm{mPW} 1 \mathrm{PW} 91 / 6-31+\mathrm{G}^{* *}\right)=-1524.12221889$

S-MPTA-3_027

B3LYP/6-31 G* Geometry

O $2.353889-1.4827110 .726086$

C $3.583862-0.272364-1.011160$

C $1.736625-1.817066-1.595062$

C $2.573308-0.845359-1.963080$

C $1.706179-2.366042-0.189677$

C $3.649887-1.0973550 .276157$

C $4.323870-0.3347001 .380522$

C 4.4344180 .9960331 .321951

C $3.7908151 .794561 \quad 0.251082$

O $3.2315561 .118547-0.788264$

H $4.236536-2.0046460 .048029$

H $4.582882-0.275247-1.470015$

C $0.297374-2.616502 \quad 0.357517$

C $-0.383275-3.852089-0.222881$

$\begin{array}{lllll}\text { O } & 3.678663 & 2.999579 & 0.308525\end{array}$

H $2.226548-3.342995-0.166770$

O $-0.519044-1.457843 \quad 0.041834$

H $1.036501-2.239850-2.310834$

H $2.551908-0.433900-2.968501$

H $4.694206-0.8974162 .233630$

H $4.909591 \quad 1.5807442 .102514$

H $\quad 0.371630-2.6936471 .444029$

H $0.186706-4.7549920 .021781$

H - $0.483976-3.783724-1.310908$

$\mathrm{H}-1.385807-3.9527040 .201718$

C -1.378941-1.029183 0.988826

O $-1.417766-1.4519992 .118787$

C -2.3742340 .0315790 .428180$

C $-3.078652-0.632281-0.798518$

$\begin{array}{lllll}\text { O } & -3.437441 & 0.194254 & 1.342409\end{array}$

C -3.173869 0.9024382 .556824

H -2.434706 0.3820193 .169543 
H -4.131572 $0.926033 \quad 3.081529$

H -2.844906 1.9286732 .359558

C -1.7380501 .3669190 .014100$

C $-0.7317553 .880077-0.729240$

C $-0.357417 \quad 1.582318-0.063760$

C $-2.6105002 .427405-0.281803$

C $-2.1106373 .673175-0.652466$

C $0.1418632 .834700-0.434455$

$\begin{array}{llll}\text { H } & 0.342894 & 0.783119 & 0.149527\end{array}$

H -3.681291 $2.270276-0.213456$

$\mathrm{H}-2.8001994 .482365-0.878423$

H $1.2151932 .989394-0.486122$

H $-0.3396114 .852564-1.014796$

F -3.420123 - $1.911854-0.516033$

F $-4.2042790 .017562-1.133731$

F -2.284109-0.653736-1.881945

SCF Energy $\left(P C M / m P W 1 P W 91 / 6-31+G^{\star *}\right)=-1524.12392832$

S-MPTA-3_029

B3LYP/6-31G* Geometry

O $3.0581121 .133219-0.005471$

C $4.585059-0.4412421 .092061$

C $2.132129-0.788761 \quad 1.149678$

C $3.359922-1.2704081 .351306$

C $1.8809190 .585274 \quad 0.581797$

C 4.2055021 .0190220 .833234

C 5.3304971 .7646290 .174849

C $6.3073401 .106072-0.456082$

C $6.289252-0.367413-0.636643$

$\begin{array}{lll}\text { O } & 5.309979-1.063891 & 0.002847\end{array}$

H $3.9830181 .473721 \quad 1.813840$

H $5.247041-0.4655651 .969943$

C $0.8233990 .587564-0.538276$

C $0.604901 \quad 1.966709-1.145060$

O $7.060405-0.946510-1.366899$

H 1.5191601 .2548871 .384697

$\begin{array}{lllll}\text { O } & -0.401144 & 0.124902 & 0.105291\end{array}$

H $1.254710-1.3789231 .397011$

H $3.515965-2.2794111 .723834$

H 5.3066592 .8510620 .206160

H $7.1312431 .612323-0.948197$

H $1.102578-0.135500-1.309073$

H $1.5439492 .332836-1.566571$

H $0.2624382 .672777-0.380534$

H $-0.1414931 .914910-1.941009$

C $-1.359945-0.368891-0.696717$

O $-1.261936-0.508863-1.894398$

C $-2.657168-0.6540310 .107222$

C $-3.509190-1.720602-0.647521$

O $-2.389857-1.0845991 .419308$

C $-1.709521-2.3236051 .612183$

H -2.379914 -3.178122 1.476341

H $-1.355186-2.3024392 .645251$

H $-0.851889-2.4328890 .938103$

C $-3.4491530 .661107 \quad 0.213839$

$\begin{array}{llll}\text { C }-4.912967 & 3.042376 & 0.422232\end{array}$

C $-3.6265331 .482924-0.907432$

C -4.0145881 .0355501 .437148$

C $-4.7419572 .222179 \quad 1.537641$

C $-4.3533072 .668117-0.800199$

H -3.202317 $1.197778-1.864206$

$\begin{array}{llll}H & -3.879021 & 0.399608 & 2.302817\end{array}$

H -5.1765702 .5031432 .493235$

H $-4.4823443 .297233-1.676618$

H $-5.479237 \quad 3.9662240 .504124$

F $-4.525868-2.1156460 .142984$

F $-4.031049-1.261228-1.793336$

F - $2.779841-2.820587-0.945885$

SCF Energy $\left(\mathrm{PCM} / \mathrm{mPW} 1 \mathrm{PW} 91 / 6-31+\mathrm{G}^{* *}\right)=-1524.12478537$

S-MPTA-3_032

B3LYP/6-31G* Geometry

O $2.406034-0.096061-0.022268$

C $4.6077510 .356506-0.998250$

C $2.7802372 .021839-1.152120$

C $4.0750311 .731103-1.290800$

C $1.7748321 .021721-0.636172$
C $3.462106-0.641416-0.806956$

C $3.939673-1.890551-0.123426$

C $5.076795-1.8898660 .579060$

C $5.867498-0.656400 \quad 0.814942$

$\begin{array}{lllll}\text { O } & 5.487503 & 0.468418 & 0.148050\end{array}$

H $3.079095-0.891809-1.811026$

H $5.2230550 .007474-1.840145$

C $0.836147 \quad 1.6537690 .407689$

C $1.496837 \quad 1.9443251 .745333$

O $6.778048-0.6028551 .610010$

H $1.147600 \quad 0.674401-1.476075$

O -0.2550050 .7227380 .667608$

H $2.4004223 .004717-1.425072$

H $4.7870062 .475730-1.636877$

H $3.315326-2.777428-0.194926$

H $5.447130-2.7702621 .093888$

H $0.4038152 .556854-0.031614$

H 2.3443452 .6220421 .602366

H 1.8680281 .0224942 .197682

H 0.7830452 .4188322 .425461

C -1.301305 $0.769266-0.166676$

O $-1.426158 \quad 1.569365-1.069897$

C $-2.313853-0.3856750 .095485$

C $-2.164577-1.307325-1.157294$

O $-1.916746-1.2294011 .154181$

C -2.045141 -0.7322762 .486334$

H -1.447239 0.1707732 .639285

H - $1.659999-1.5291893 .125871$

H -3.090579 -0.5325232 .745315$

$\begin{array}{llll}\text { C }-3.774985 & 0.078993 & 0.224859\end{array}$

C -6.4836440 .7684160 .526778$

C $-4.216046 \quad 1.371748-0.080226$

C $-4.709740-0.8673790 .675490$

C $-6.051860-0.5253330 .825254$

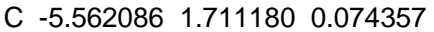

H -3.519302 $2.111322-0.453585$

H -4.376663 -1.872077 0.911136

H $-6.759728-1.2715581 .175816$

H $-5.8869482 .719939-0.165900$

H -7.529994 1.0371630 .644168

F - $2.803289-2.475579-0.988667$

F -2.656012 - $0.719489-2.257535$

F $-0.860680-1.586088-1.385931$

SCF Energy $\left(\mathrm{PCM} / \mathrm{mPW} 1 \mathrm{PW} 91 / 6-31+\mathrm{G}^{* *}\right)=-1524.12421941$

S-MPTA-3_034

B3LYP/6-31G* Geometry

O $3.005730-1.113447-0.362362$

C $4.0625691 .051424-0.823019$

C $1.5957120 .832868-0.719512$

C $2.6740501 .618265-0.747133$

C $1.689657-0.671799-0.685589$

C $4.019307-0.450731-1.114893$

C $5.331420-1.105635-0.791117$

C $6.205945-0.5115860 .026403$

C $5.899867 \quad 0.7548250 .737869$

O 4.7423791 .3917610 .411374

H $3.803716-0.564322-2.191141$

H $4.6211891 .535513-1.637253$

C $0.775465-1.303608 \quad 0.380599$

C $0.854875-2.8234350 .413060$

O 6.5991821 .1939621 .622175

H $1.390199-1.079567-1.669352$

O $-0.569425-0.8827170 .009295$

H $0.595181 \quad 1.256321-0.726992$

H $2.5826412 .701235-0.733961$

H $5.530098-2.084705-1.219908$

H $7.159322-0.9586700 .288029$

H $1.011472-0.8804001 .360567$

H $1.883552-3.131826 \quad 0.614821$

H $0.541818-3.242840-0.549392$

H $0.206454-3.218678 \quad 1.198629$

C - $1.516621-0.9086230 .963455$

O $-1.324529-1.1977372 .120060$

C $-2.907997-0.5001360 .389519$

C $-3.162328-1.385589-0.870012$

O $-3.928393-0.916797 \quad 1.270674$ 
C $-4.145687-0.1572992 .461055$ H -3.256395 -0.1399103 .095914$ H $-4.952768-0.6773892 .981328$ H -4.4652850 .8658722 .231651$ C -2.9580230 .9997620 .066402$ C $-3.1461353 .766201-0.414478$ C $-2.027733 \quad 1.888896 \quad 0.620724$ C $-4.000606 \quad 1.520452-0.714885$ C $-4.0879002 .890404-0.957638$ C $-2.118307 \quad 3.2613390 .380725$ H $-1.2294531 .520978 \quad 1.258485$ H $-4.7452630 .855734-1.136245$ H $-4.8979403 .273223-1.572367$ H $-1.386740 \quad 3.9321360 .822679$ H -3.217517 $4.833563-0.604484$ F -4.465422 -1.410500 -1.207220 F -2.477577 -0.950585-1.941773 F -2.790224-2.660295 -0.627668 SCF Energy $\left(\mathrm{PCM} / \mathrm{mPW} 1 \mathrm{PW} 91 / 6-31+\mathrm{G}^{* *}\right)=-1524.12374138$

S-MPTA-3_040

B3LYP/6-31G* Geometry

O $-2.2927490 .045697-0.136353$

C $-4.422226-0.513322-1.216267$

C $-2.690481-2.244916-0.844104$

C $-3.947979-1.938516-1.167976$

C $-1.677795-1.200034-0.443698$

C $-3.2415660 .457327-1.115541$

C $-3.7042181 .838384-0.749459$

C $-4.8998862 .028432-0.183273$

C -5.7830570 .9034610 .211918$

O $-5.409651-0.349859-0.168817$

H -2.756302 $0.480037-2.106540$

H $-4.934332-0.322967-2.170675$

C $-0.876380-1.6398750 .795050$

C $-1.676732-1.6325962 .087282$

O -6.7673901 .0523150 .899476$

H $-0.959194-1.062311-1.270922$

$\begin{array}{lllll}\text { O } & 0.234842 & -0.718097 & 0.988412\end{array}$

H -2.346982 -3.277432 -0.871368

H -4.669111 - 2.710213-1.424115

H $-3.0227202 .665477-0.930803$

H $-5.2619263 .010306 \quad 0.103185$

H $-0.454577-2.6274040 .587144$

H $-2.543436-2.2942811 .993034$

H $-2.034779-0.6251662 .308293$

H $-1.057648-1.9833922 .918220$

C $1.340843-0.9279920 .258605$

O $1.493902-1.861115-0.497548$

C $2.3987090 .187797 \quad 0.552281$

C 1.7950981 .5489540 .090856

$\begin{array}{lllll}\text { O } & 2.569421 & 0.348614 & 1.947123\end{array}$

C $2.885895-0.8319542 .681260$

H $3.760486-1.3485702 .269255$

H $2.033191-1.5215282 .711419$

H $3.106290-0.4972263 .696870$

C $3.729103-0.026209-0.184253$

C $6.206453-0.244068-1.493911$

C $3.795633-0.484962-1.506963$

C 4.9142720 .3532730 .459886

C $6.1445730 .236268-0.185668$

C $5.027860-0.595745-2.151969$

H $2.892928-0.764165-2.033151$

H $4.8654720 .754883 \quad 1.465884$

H 7.0529780 .5299820 .333439

H $5.062132-0.959459-3.175387$

H $7.164163-0.335523-1.999313$

F $2.718007 \quad 2.5237770 .126869$

F $1.3626781 .432060-1.190072$

F $0.760167 \quad 1.9448600 .837963$

SCF Energy $\left(P C M / m P W 1 P W 91 / 6-31+G^{* *}\right)=-1524.12332262$

S-MPTA-3_043

B3LYP/6-31G* Geometry

$\begin{array}{llll}O & -3.233243 & -1.180156 & 0.386428\end{array}$

$\begin{array}{llll}\text { C }-4.526647 & 0.884294 & 0.687424\end{array}$

C $-2.049255 \quad 0.905034 \quad 0.784634$
C $-3.1959301 .580574 \quad 0.691834$

C -2.002256 -0.6027190 .811021$

C $-4.366221-0.5992921 .029616$

C $-5.578941-1.3868980 .624976$

C $-6.440219-0.899373-0.272989$

C $-6.2034040 .375905-0.995531$

$\begin{array}{llll}0 & -5.140716 & 1.128809 & -0.603035\end{array}$

H $-4.231322-0.6645242 .123119$

H -5.1883301 .3324951 .442890$

C $-0.925917-1.166279-0.134847$

C $-0.885255-2.686102-0.181747$

O $-6.8729330 .724839-1.940992$

H $-1.764518-0.949126 \quad 1.834853$

$\begin{array}{lllll}\text { O } & 0.327048 & -0.651342 & 0.403159\end{array}$

H $-1.094814 \quad 1.4214610 .833288$

H -3.2065872 .6653310 .626925$

H $-5.715381-2.3713231 .065812$

H -7.322986 -1.443379 -0.592530

H $-1.064160-0.753410-1.136729$

H - $1.845163-3.067855-0.537938$

H $-0.692374-3.1011840 .814089$

H $-0.098812-3.019623-0.864363$

C $1.314301-0.390479-0.467701$

O $1.254891-0.609331-1.656340$

C $2.519103 \quad 0.3152910 .228388$

C $2.433427 \quad 1.796835-0.257216$

O $2.3419180 .432323 \quad 1.624762$

C $2.471398-0.7523882 .410333$

H $1.723947-1.5024702 .134155$

H $2.296421-0.4330353 .439983$

H $3.474266-1.1851722 .331013$

C $3.895669-0.263197-0.146214$

C $6.484534-1.207076-0.705771$

C $4.116938-1.131711-1.221417$

C $4.990305 \quad 0.1364200 .638092$

C $6.272500-0.3327430 .361792$

C $5.404185-1.600790-1.493454$

H $3.294074-1.430949-1.857392$

H $4.8293370 .816898 \quad 1.466892$

H $7.105828-0.0124610 .981305$

H $5.557227-2.275053-2.331740$

H $7.484019-1.574179-0.922774$

F 3.3001452 .5812740 .400578

F $2.6926021 .891956-1.568057$

F $1.1932202 .297278-0.044751$

SCF Energy $\left(\mathrm{PCM} / \mathrm{mPW} 1 \mathrm{PW} 91 / 6-31+\mathrm{G}^{* *}\right)=-1524.12369424$

S-MPTA-3_044

B3LYP/6-31 G* Geometry

O $-3.1054690 .989143-0.715124$

C $-4.493658-0.987562-0.273604$

C $-2.018072-1.134977-0.291934$

C $-3.194688-1.6826540 .016656$

C $-1.9015030 .246766-0.888469$

C $-4.2635620 .264600-1.125243$

C $-5.4388431 .196243-1.056854$

C $-6.3303281 .099730-0.066046$

C -6.1638650 .1580951 .069436$

O $-5.135157-0.7294010 .999992$

H - $-4.122884-0.075712-2.165894$

H $-5.162191-1.656440-0.835029$

C $-0.7839751 .084565-0.236059$

C $-0.7480702 .526425-0.717747$

$\begin{array}{llll}\text { O } & -6.859436 & 0.197550 & 2.058467\end{array}$

H $-1.6680160 .171759-1.967667$

\begin{tabular}{lllll}
\hline & 0.479637 & 0.470090 & -0.629484
\end{tabular}

H - $1.095860-1.675028-0.095749$

H -3.254234 -2.6578860 .492303$

H $-5.5230641 .962681-1.823326$

H $-7.187624 \quad 1.7598090 .015512$

H $-0.872181 \quad 1.0278150 .850382$

H - $1.6787523 .030694-0.448092$

H $-0.6254822 .571384-1.805370$

H $0.0904763 .053644-0.252919$

C $1.226226-0.1138600 .322872$

O $0.897916-0.2624991 .478327$

C $2.618405-0.525754-0.227943$ 
C $3.066043-1.8354720 .492340$

O $2.628011-0.708276-1.621200$

C $1.865724-1.764010-2.203773$

H $2.322991-2.742187-2.025237$

H $1.866098-1.556411-3.275984$

H $\quad 0.831360-1.772061-1.844285$

C $3.6138800 .609623 \quad 0.080534$

C 5.4583192 .6515420 .619359

C $4.5133921 .026673-0.906918$

C 3.6463571 .2187241 .342455

C 4.5640802 .2350381 .606300

C $5.4296342 .043345-0.635738$

H $4.4890460 .556523-1.881610$

H 2.9579710 .9008192 .117180

H 4.5777362 .6999522 .588259

H $6.1228432 .358331-1.411114$

H 6.1721153 .4440730 .827431

F $4.151267-2.340861-0.123053$

F $3.370019-1.6535161 .783565$

F $2.092344-2.7786340 .434558$

SCF Energy $\left(P C M / m P W 1 P W 91 / 6-31+G^{* *}\right)=-1524.12351870$

S-MPTA-3_045

B3LYP/6-31 G* Geometry

O $2.775235-1.961761-1.110807$

C $4.0911200 .093886-1.201871$

C $1.6122190 .200687-1.262518$

C $2.7846070 .832486-1.297729$

C $1.507788-1.295169-1.150305$

C $3.890693-1.278505-0.536868$

C $3.811763-1.1340300 .961723$

C $4.344189-0.0795861 .587281$

C 5.0792990 .9749430 .850098

O $5.077126 \quad 0.901679-0.514866$

H $4.738462-1.933055-0.776855$

H $4.524280-0.042615-2.198122$

C $0.630123-1.7467540 .042735$

C $0.464842-3.2563410 .137366$

O 5.7220021 .8411001 .396368

H $1.023941-1.680972-2.058985$

O $-0.667832-1.130693-0.208905$

H $0.6751210 .745097-1.332342$

H $2.8476541 .912345-1.407336$

H $3.349247-1.9480791 .515805$

H 4.3307200 .0358222 .665810

H $1.015718-1.3348530 .978381$

H $1.443336-3.7344870 .231920$

H $-0.022669-3.644492-0.763612$

H $-0.142745-3.5165791 .008420$

C $-1.425140-0.813808 \quad 0.853594$

$\begin{array}{llll}\text { O } & -1.129908 & -1.027837 & 2.006168\end{array}$

C $-2.713887-0.047256 \quad 0.424152$

C $-3.408569-0.793035-0.748656$

O -3.6038790 .0782801 .507393$

C $-4.160499-1.0885542 .125827$

H -4.970662 -1.511359 1.522578

H -4.567454 -0.7339063 .075012$

H -3.402714 -1.850226 2.323881

C -2.2920541 .3959310 .094221$

C $-1.4933024 .062546-0.283328$

C -1.7300382 .1308201 .149759$

C $-2.4572012 .013678-1.150915$

C $-2.0587923 .340598-1.332999$

C $-1.3316443 .451966 \quad 0.961973$

H $-1.615803 \quad 1.6607392 .120863$

H $-2.8978861 .482993-1.984319$

$\mathrm{H}-2.198776 \quad 3.807841-2.303956$

H -0.8970434 .0044291 .790415$

$\mathrm{H}-1.1845195 .093618-0.432193$

F -4.626887 $-0.274621-0.978798$

F -2.723976 - $0.756986-1.908656$

F $-3.571721-2.102329-0.436926$

SCF Energy $\left(\mathrm{PCM} / \mathrm{mPW} 1 \mathrm{PW} 91 / 6-31+\mathrm{G}^{* *}\right)=-1524.11678268$

S-MPTA-3_047

B3LYP/6-31G* Geometry

O $2.932412-1.052676-0.471480$
C $3.9944701 .155015-0.414879$

C $1.5605290 .899803-0.016521$

C 2.6544831 .6623370 .034810

C $1.597726-0.541799-0.459008$

C $3.851376-0.187667-1.135166$

C $5.169930-0.900999-1.223561$

C $6.174256-0.579525-0.402491$

C 6.0207330 .4061390 .697063

$\begin{array}{lllll} & \text { O } & 4.856168 & 1.107385 & 0.750775\end{array}$

H $3.4772940 .027685-2.150499$

H $4.4503981 .867534-1.117564$

C $0.844814-1.4763420 .514686$

C $0.861387-2.942890 \quad 0.100317$

$\begin{array}{lllll}\text { O } & 6.853514 & 0.555090 & 1.562307\end{array}$

H $1.154744-0.641734-1.462222$

O $-0.510712-0.992953 \quad 0.774155$

H $0.592348 \quad 1.3038920 .266548$

H 2.6089852 .6869190 .394295

H $5.267561-1.700263-1.954046$

H $7.137366-1.078393-0.432268$

H $1.316827-1.3644361 .493907$

H $1.897843-3.288000 \quad 0.071619$

H $0.411831-3.088352-0.884031$

H $\quad 0.315754-3.5450950 .834238$

C -1.420994-0.955949 -0.207794

O $-1.232476-1.253440-1.367446$

C $-2.766120-0.3793550 .316901$

C $-3.938531-0.992771-0.510221$

O $-2.965936-0.6181501 .686294$

C -3.075652 -1.962331 2.154905

H -4.052323 -2.394552 1.916217

H -2.963258 -1.8972783 .239096$

H -2.283991 -2.603409 1.752722

C $-2.733347 \quad 1.1493830 .126514$

C -2.704292 $3.934451-0.200901$

C $-2.320021 \quad 1.715339-1.088038$

C $-3.132741 \quad 1.987191 \quad 1.173669$

C -3.117012 3.3725501 .007447

C -2.306148 $3.101062-1.247043$

H $-2.0146201 .076699-1.909259$

H -3.4503101 .5503662 .111728$

H -3.429850 4.0124331 .828113

H - $-1.9846753 .526833-2.193637$

H -2.692935 $5.013704-0.327049$

F -5.109498 -0.6705340 .069905$

F $-3.975444-0.563641-1.779942$

F $-3.860817-2.344540-0.543173$

SCF Energy $\left(\mathrm{PCM} / \mathrm{mPW} 1 \mathrm{PW} 91 / 6-31+\mathrm{G}^{* *}\right)=-1524.12124542$

S-MPTA-3_048

B3LYP/6-31G* Geometry

O -2.301246 -0.190716-0.146994

C $-4.634435-0.8172340 .267553$

C $-2.879530-1.2701211 .954206$

C $-4.175464-1.2038561 .644682$

C -1.789398 -0.9040330 .978000$

C $-3.459142-0.801662-0.713705$

C $-3.810936-0.049709-1.965295$

C $-4.821353 \quad 0.825137-1.971577$

C $-5.5753821 .180797-0.743333$

$\begin{array}{llll}\text { O } & -5.320327 & 0.454486 & 0.379693\end{array}$

H -3.230536-1.851625 - 0.963105

H $-5.370324-1.546709-0.100269$

C $-0.716601-0.0226931 .642662$

C -1.198152 1.3667382 .029141

O $-6.3447892 .112952-0.694083$

H -1.291669-1.825754 0.632115

$\begin{array}{lllll} & \mathrm{H} & 0.391324 & 0.167819 & 0.718921\end{array}$

H -2.561605 -1.609274 2.938322

H $-4.943406-1.4442312 .375016$

H -3.203229 $-0.221898-2.850117$

H $-5.0991961 .392364-2.853818$

H $-0.332875-0.5697252 .509182$

H -2.008388 1.2932472 .761083

H -1.573683 1.9009621 .153705

H -0.3774421 .9381672 .472004$

C $1.290685-0.8282040 .615624$ 
O $1.251778-1.852807 \quad 1.258278$

C $2.365510-0.473735-0.449560$

C $3.314640-1.695608-0.614028$

O $1.788013-0.361704-1.738927$

C $0.861356 \quad 0.693447-2.019345$

H $1.1859331 .647536-1.592928$

H $-0.1393770 .456672-1.648310$

H $0.8449270 .770538-3.109703$

C $3.1951390 .744434-0.031615$

C 4.8199292 .9119480 .692900

C 3.3984561 .0578211 .316962

C $3.8301131 .512798-1.014381$

C $4.6319862 .595094-0.653422$

C 4.2053552 .1368541 .677291

H 2.9386540 .4534232 .094069

H $3.6988351 .252164-2.059092$

H $5.1152203 .186843-1.426093$

H 4.3543632 .3690772 .728185

H 5.4456263 .7547500 .973670

F $4.241598-1.438147-1.557258$

F $3.963505-1.9554380 .535051$

F $2.651952-2.799308-0.989523$

SCF Energy $\left(\mathrm{PCM} / \mathrm{mPW} 1 \mathrm{PW} 91 / 6-31+\mathrm{G}^{* *}\right)=-1524.12448577$

S-MPTA-3_049

B3LYP/6-31 G* Geometry

O $-3.263928-1.035824-0.323453$

C -4.2802960 .6305801 .162103$

C -1.8360550 .2209831 .192983$

C $-2.872690 \quad 0.980993 \quad 1.550605$

C $-1.989086-0.993504 \quad 0.311807$

C $-4.344683-0.7798780 .571296$

C $-5.625031-0.995191-0.183573$

C $-6.3466520 .045341-0.610813$

C $-5.8843951 .448999-0.467573$

O $-4.759667 \quad 1.6652850 .266721$

H -4.289688 -1.486741 1.416828

H -4.9298200 .6525462 .049270$

C $-0.962898-1.024912-0.836666$

C $-1.087840-2.254829-1.723752$

O $-6.4284762 .376057-1.023065$

H $-1.843653-1.9069130 .918632$

O $0.332088-1.016576-0.169228$

H $-0.8299030 .445604 \quad 1.534714$

H -2.7340801 .8727672 .156150$

H -5.926752 -2.018820 -0.391183

H $-7.272360-0.072220-1.164490$

H $-1.050047-0.110019-1.428325$

H $-2.086255-2.285382-2.167073$

H $-0.934154-3.168488-1.138890$

H $-0.347422-2.216019-2.526599$

C $1.377383-0.510405-0.838622$

O $1.334896-0.112602-1.981481$

C $2.707213-0.582524-0.032815$

C $2.462659-0.7365571 .495001$

O $3.367311-1.792977-0.376906$

C $3.710014-1.993959-1.750960$

H $2.823136-2.178610-2.366733$

H $4.348941-2.879490-1.756928$

H $4.256640-1.139829-2.163146$

C $3.5690430 .663794-0.263419$

C $5.2058552 .925831-0.540778$

C $3.0049481 .921264-0.505446$

C $4.9602320 .553293-0.144996$

C $5.7739741 .675737-0.290776$

C $3.8196673 .045269-0.643163$

H $1.9302012 .032000-0.600798$

H $5.399187-0.4154750 .068250$

H $6.8521141 .572845-0.202754$

H $3.3670514 .013973-0.835682$

H $5.8391823 .801495-0.654116$

F $3.625318-0.7049112 .169822$

F $1.704466 \quad 0.294650 \quad 1.944607$

F $1.848185-1.8810801 .819960$

SCF Energy $\left(\mathrm{PCM} / \mathrm{mPW} 1 \mathrm{PW} 91 / 6-31+\mathrm{G}^{* *}\right)=-1524.12371387$

S-MPTA-3_058
B3LYP/6-31G* Geometry

O $2.437183-0.3139170 .395090$

C $4.805176-0.8539920 .044305$

C $3.029790-2.017321-1.232694$

C $4.324295-1.738970-1.070898$

C $1.953723-1.420423-0.356957$

C $3.677969-0.5761361 .043069$

C $4.000900 \quad 0.6078151 .908982$

C 4.9254381 .4962861 .531946

C 5.6003201 .4339800 .211519

$\begin{array}{llll}\text { O } & 5.364225 & 0.337165 & -0.560663\end{array}$

H $3.576733-1.4776051 .672270$

H $5.622064-1.3498850 .588528$

C $0.757845-0.944716-1.190809$

C $1.0328800 .289990-2.038491$

O $6.2859152 .333430-0.217566$

H $1.579674-2.1973130 .336568$

O $-0.310698-0.651961-0.243104$

H $2.704759-2.703093-2.013052$

H $5.078290-2.154586-1.734078$

H 3.4380070 .7302642 .830766

H 5.1775152 .3668962 .128375

H $\quad 0.421479-1.775034-1.818779$

H $1.8597620 .094740-2.729069$

H $1.3054661 .134789-1.402641$

H $0.1458030 .546095-2.623924$

C $-1.563941-0.875737-0.665205$

O $-1.864578-1.347748-1.739853$

C $-2.602433-0.375870 \quad 0.373725$

C $-3.885418-1.2575310 .274651$

O $-2.108527-0.3885461 .689289$

C $-1.743943-1.6301262 .289496$

H -1.109981-2.234382 1.631367

H -2.623146 -2.211837 2.583710

H -1.174252 -1.360124 3.181322

C $-2.945361 \quad 1.0875350 .035291$

C -3.590518 $3.752292-0.550062$

C $-2.9784882 .045262 \quad 1.054467$

C $-3.2391931 .471755-1.280332$

C $-3.5589542 .798234-1.567973$

C $-3.299714 \quad 3.370703 \quad 0.759731$

H $-2.748611 \quad 1.7493022 .069963$

H $-3.2197410 .737994-2.078386$

H -3.783770 $3.083492-2.592037$

H $-3.321490 \quad 4.1056901 .559920$

H $-3.8388754 .785657-0.776753$

F $-4.706096-0.971173 \quad 1.302785$

F $-4.570062-1.074826-0.862684$

F $-3.581628-2.5758500 .355213$

SCF Energy $\left(\mathrm{PCM} / \mathrm{mPW} 1 \mathrm{PW} 91 / 6-31+\mathrm{G}^{* *}\right)=-1524.12544167$

S-MPTA-3 064

B3LYP/6-31 G* Geometry

O $-2.2046190 .346413-0.113952$

C $-4.4931980 .777395-0.889989$

C $-2.808978-0.253479-2.387052$

C $-4.0840940 .050479-2.139994$

C $-1.6985620 .049910-1.409168$

C $-3.2655531 .296319-0.134332$

C $-3.6052891 .633991 \quad 1.288712$

C -4.7054921 .1388591 .863687$

C -5.5872590 .1558991 .186710$

O $-5.325174-0.123863-0.120634$

H $-2.9270742 .206928-0.658945$

H $-5.117817 \quad 1.644227-1.151002$

C $-0.727333-1.129724-1.282417$

C $-1.297433-2.337955-0.551290$

O $-6.469912-0.438853 \quad 1.761401$

H - $-1.1170830 .915575-1.779887$

O $0.430972-0.623689-0.553088$

H $-2.525743-0.732408-3.322593$

H $-4.872029-0.206746-2.842941$

H -2.912385 $2.266171 \quad 1.837972$

H -4.9761291 .3564032 .891609$

H $-0.386714-1.409091-2.284018$

H -2.201916 - $2.690710-1.057417$

H -1.556562 -2.0708190 .475192$ 
H $-0.564791-3.148862-0.542174$

C $1.610950-1.221828-0.795370$

O $1.787870-2.068812-1.639360$

C $2.730229-0.715027 \quad 0.165274$

C $2.186975-0.854647 \quad 1.623133$

O $3.810828-1.622247 \quad 0.150774$

C $4.648210-1.667317-1.008443$

H $4.097228-2.014189-1.884501$

H $5.432316-2.385636-0.759154$

H $5.106029-0.692662-1.212353$

C $3.1723030 .733494-0.096548$

C $4.1133653 .350181-0.510615$

C $2.5060441 .599107-0.970019$

C 4.3216971 .1939440 .565108

C 4.7858352 .4915920 .361593

C $2.9747262 .898616-1.175587$

H $1.6120361 .272693-1.488128$

H 4.8503610 .5263561 .236055

H 5.6759942 .8309150 .884260

H $2.4451923 .556260-1.859714$

H $4.4765424 .361542-0.671670$

F $3.181941-0.7736562 .523943$

F $1.2958640 .104761 \quad 1.917841$

F $1.584414-2.0507051 .790207$

SCF Energy $\left(\mathrm{PCM} / \mathrm{mPW} 1 \mathrm{PW} 91 / 6-31+\mathrm{G}^{* *}\right)=-1524.12351319$

S-MPTA-3_069

B3LYP/6-31G* Geometry

O $3.021293-0.547203-0.891814$

$\begin{array}{llll}\text { C } 4.210782 & 1.370771 & 0.073247\end{array}$

C 1.7611031 .1064310 .351801

$\begin{array}{llll}\text { C } 2.895566 & 1.706485 & 0.716011\end{array}$

C $1.7242930 .002453-0.677111$

C $4.0074270 .453485-1.136230$

C $5.283679-0.239251-1.517665$

C $6.296396-0.331468-0.650618$

C $6.1903130 .137396 \quad 0.753867$

$\begin{array}{llll}\text { O } & 5.061036 & 0.811284 & 1.104327\end{array}$

H $3.6707681 .091944-1.971472$

H $4.7030022 .288400-0.280595$

C $0.824524-1.172805-0.251974$

C $0.901263-2.370361-1.186894$

O $7.027247-0.110787 \quad 1.591309$

H $1.3282700 .391723-1.634468$

O $-0.552127-0.685885-0.303400$

$\begin{array}{lllll}\text { H } & 0.818902 & 1.388004 & 0.814347\end{array}$

H 2.9082212 .4627621 .496299

H $5.343041-0.681921-2.508854$

H $7.229809-0.829018-0.892425$

H $1.050896-1.4533350 .778264$

H $1.906427-2.797363-1.166334$

H $\quad 0.663235-2.076296-2.214784$

H $0.180186-3.129525-0.870211$

C -1.282052 -0.6908200 .831086$

O $-0.828645-0.8435651 .938905$

C $-2.807134-0.5260190 .538640$

C $-3.192369-1.642919-0.484256$

O $-3.544904-0.8876541 .684213$

C -3.520734 -0.009926 2.814639

H -2.520789 $0.053198 \quad 3.247473$

H -4.204774 -0.4631153 .535279$

H -3.8840460 .9906442 .554305$

C $-3.2153590 .848966-0.015014$

C $-4.1087403 .333033-0.982849$

C $-2.3031311 .798906-0.485642$

C $-4.5847021 .158244-0.037015$

C $-5.0265142 .388902-0.517834$

C $-2.7478123 .033145-0.964696$

H $-1.2427661 .579237-0.499125$

H -5.2994600 .4296550 .329210$

H -6.090302 $2.610076-0.527421$

H -2.023609 3.759206-1.324104

H $-4.4529274 .293910-1.355465$

F -4.521725 -1.823176 - 0.532748

F - $2.777094-1.345797-1.726531$

F -2.634077 -2.822759-0.132632

SCF Energy $\left(\mathrm{PCM} / \mathrm{mPW} 1 \mathrm{PW} 91 / 6-31+\mathrm{G}^{* *}\right)=-1524.12117011$
S-MPTA-3_071

B3LYP/6-31G* Geometry

O $-2.3330860 .094345-0.112185$

C $-4.615922-0.228207-0.946266$

C $-2.765842-1.678779-1.722012$

C $-4.076629-1.441602-1.649109$

C $-1.739970-0.796743-1.056086$

C $-3.4900510 .756449-0.620270$

C -3.9328431 .7607060 .405318$

C $-4.977696 \quad 1.5064251 .198941$

C $-5.685967 \quad 0.201773 \quad 1.190655$

O $-5.346636-0.6880090 .217676$

H -3.230547 $1.274084-1.559413$

H $-5.3383090 .288912-1.594207$

C $-0.686690-1.634315-0.306492$

C - $1.233626-2.4112220 .880439$

O $-6.490938-0.1116812 .037463$

H $-1.211140-0.212680-1.827502$

O $0.343266-0.7554510 .226901$

H -2.387260 -2.529919-2.285057

H - $-4.798063-2.109543-2.112014$

H -3.3620872 .6814840 .496337$

H $-5.3209232 .203321 \quad 1.956428$

H $-0.211360-2.296756-1.036920$

H - $1.986287-3.1315030 .545408$

H -1.700819-1.733254 1.598102

H -0.424884 -2.955072 1.376489

C $1.267255-0.301607-0.628492$

O $1.311878-0.569352-1.810838$

C 2.3251260 .5724690 .096105

C $2.784277 \quad 1.701716-0.877967$

$\begin{array}{lllll}\text { O } & 1.849989 & 1.132590 & 1.292878\end{array}$

C $0.741962 \quad 2.0363101 .267931$

H -0.1179001 .6159410 .737104$

H 1.0182853 .0029620 .835331

H 0.4733952 .1763102 .317209

C $3.525160-0.3213460 .465596$

C $5.723909-1.9156021 .162301$

C $4.069422-1.220235-0.461827$

C $4.091499-0.2236361 .741349$

C 5.185349-1.018702 2.085038

C $5.162167-2.012635-0.111374$

H $3.641713-1.302168-1.454554$

H $3.670540 \quad 0.4697842 .457983$

H $5.615765-0.934103 \quad 3.079315$

H $5.573682-2.706788-0.839078$

H $6.574951-2.5347291 .433263$

F $3.5239772 .603174-0.207172$

F $3.5153341 .251324-1.907220$

F $1.7184832 .359599-1.399168$

SCF Energy $\left(\mathrm{PCM} / \mathrm{mPW} 1 \mathrm{PW} 91 / 6-31+\mathrm{G}^{* *}\right)=-1524.12589027$

S-MPTA-3_090

B3LYP/6-31 G* Geometry

O $-3.060220-1.251520-0.062922$

C $-4.0175140 .660282 \quad 1.137536$

C $-1.582384 \quad 0.207012 \quad 1.210755$

C $-2.599621 \quad 1.0327301 .464475$

C -1.766137-1.119959 0.520769

C $-4.119681-0.827240 \quad 0.792458$

C $-5.415097-1.1331490 .096235$

C $-6.110927-0.161343-0.501689$

C $-5.608024 \quad 1.231777-0.605525$

$\begin{array}{lllll}\text { O } & -4.472587 & 1.539770 & 0.079399\end{array}$

H -4.066988 -1.3848711 .742919$

H -4.6629410 .8475092 .007877$

C $-0.791566-1.346402-0.653844$

C $-0.862712-2.766136-1.199203$

O $-6.1287232 .064276-1.312076$

H - $-1.604969-1.929721 \quad 1.257437$

O $0.531085-1.056403-0.115683$

H $-0.570546 \quad 0.4489201 .521630$

H -2.4364571 .9951951 .942132$

H $-5.748075-2.1677360 .071115$

H -7.045133 $-0.345361-1.021963$

H - $1.005850-0.615353-1.438432$ 
H - $1.893274-2.987512-1.489388$

H $-0.552370-3.484569-0.431874$

H $-0.217669-2.873682-2.073117$

C $1.531176-0.835002-0.987992$

O $1.436733-0.916228-2.187950$

C $2.853791-0.412765-0.282032$

C $2.919303-0.9020501 .196215$

O $3.954514-0.914782-0.999891$

C $4.078326-2.324617-1.192262$

H $4.377375-2.837490-0.272358$

H $4.862868-2.441195-1.942503$

H $3.153028-2.765246-1.578401$

C $2.9804131 .119578-0.305183$

C $3.2755603 .907906-0.302856$

C $4.2488021 .695412-0.440676$

C $1.8635551 .949859-0.158369$

C $2.0106543 .336966-0.162638$

C $4.3923523 .082571-0.440474$

H $5.1137521 .054297-0.556570$

H $0.8713531 .525209-0.048323$

H $1.1328843 .968768-0.059083$

H $5.3819853 .517643-0.550573$

H $3.3896464 .988489-0.307594$

F $4.161300-0.7237521 .679664$

F $2.069336-0.2342732 .001519$

F $2.630846-2.2205071 .308358$

SCF Energy $\left(\mathrm{PCM} / \mathrm{mPW} 1 \mathrm{PW} 91 / 6-31+\mathrm{G}^{\star *}\right)=-1524.12293479$

S-MPTA-3_093

B3LYP/6-31G* Geometry

O $-1.952444-0.040979-0.167168$

C $-4.237693-0.070807-1.056597$

C $-2.606754-1.824270-1.686021$

C $-3.868609-1.391935-1.669455$

C -1.476090 -1.061151-1.041957

C $-2.9859280 .754304-0.744555$

$\begin{array}{llll}\text { C } & -3.298066 & 1.866746 & 0.214342\end{array}$

C -4.3906921 .8184300 .982380$

C $-5.291610 \quad 0.639056 \quad 1.013350$

O $-5.064002-0.3463550 .101490$

H -2.629244 $1.176497-1.699348$

H $-4.8546890 .507998-1.759001$

C $-0.570384-1.993366-0.218113$

C -1.215990 -2.542548 1.043680

O -6.1611510 .5004331 .842719$

H $-0.852510-0.607699-1.831628$

O $0.610418-1.255471 \quad 0.210853$

H $-2.346557-2.756089-2.184671$

H $-4.671210-1.974599-2.113623$

H -2.593968 2.6925110 .280310

H -4.6423212 .5994461 .692251$

H $-0.231156-2.796097-0.879229$

H -2.109671 -3.119775 0.786902

H $-1.511478-1.7284641 .708911$

H $-0.514181-3.196177 \quad 1.569320$

C $1.623214-1.143645-0.664621$

O $1.630513-1.613619-1.778714$

C $2.800466-0.299502-0.094927$

C $3.026679-0.5962801 .420122$

O $3.971333-0.578499-0.816196$

C $4.516821-1.899480-0.796597$

H $5.009957-2.1168660 .156410$

H $5.259174-1.910308-1.597203$

H $3.757416-2.657878-1.006672$

C $2.5015631 .199532-0.294971$

C $2.0273163 .943363-0.645464$

C $1.265731 \quad 1.7552800 .065477$

C $3.4954352 .027713-0.829481$

C $3.2568103 .390980-1.004126$

C $1.0360343 .119987-0.111111$

H 0.4801381 .1329210 .477514

H $4.4487161 .598536-1.109311$

H $4.0379824 .020993-1.420967$

H 0.0759523 .5408490 .177172

H $1.8433965 .005870-0.780802$

F $4.217153-0.1037991 .806515$

F $2.085509-0.0628262 .213787$
F $3.043716-1.9322871 .657659$

SCF Energy $\left(P C M / m P W 1 P W 91 / 6-31+G^{* *}\right)=-1524.12563213$

S-MPTA-3_095

B3LYP/6-31G* Geometry

O $-2.524627-0.138846-0.368583$

C $-4.904958-0.701151-0.194151$

C -3.183009-2.090677 0.920220

C $-4.468930-1.7706660 .767292$

C $-2.074412-1.368606 \quad 0.194197$

C $-3.743575-0.272327-1.095612$

C $-4.0297521 .044747-1.757834$

C $-4.9526411 .872099-1.257920$

C -5.6647551 .5969500 .015020$

$\begin{array}{llll}O & -5.471481 & 0.377693 & 0.589569\end{array}$

H -3.623922 -1.055155-1.864076

H $-5.708780-1.083866-0.839559$

C $-0.899715-1.0520321 .127374$

C $-1.1991920 .002617 \quad 2.184758$

O $-6.345797 \quad 2.4239320 .576711$

H -1.682277 - $2.014724-0.612376$

$\begin{array}{lllll}\text { O } & 0.168027 & -0.595210 & 0.249425\end{array}$

H -2.889906 -2.904711 1.580682

H -5.248325 -2.283879 1.324193

H $-3.4438001 .310424-2.634122$

H -5.179154 $2.834734-1.704317$

H - $0.563106-1.9813321 .597405$

H -2.031714 -0.3249642 .816020$

H -1.478295 0.9478371 .713973

H -0.3225600 .1526462 .819647$

C $1.427153-0.7874010 .661303$

O $1.733209-1.3089501 .711452$

C $2.459404-0.294910-0.396014$

C $3.261560-1.567851-0.809250$

O $1.865903 \quad 0.101871-1.612481$

C $1.0828491 .299482-1.646769$

H $1.5047792 .081256-1.006366$

H $\quad 0.0501661 .097912-1.352467$

H $1.1136021 .640072-2.685142$

C 3.4357020 .7606530 .157574

C 5.2943582 .6853861 .016079

C 3.6680390 .9620331 .523183

C $4.1581071 .522666-0.773680$

C $5.0776832 .478728-0.347474$

C $4.5890051 .922928 \quad 1.945961$

H 3.1452060 .3621172 .257565

H $3.9942501 .359837-1.833064$

H $5.6265023 .060591-1.083097$

H 4.7550532 .0689273 .009880

H 6.0103493 .4317011 .349634

F $4.071974-1.312697-1.848915$

F $4.015478-2.0224730 .201960$

F $2.420608-2.558092-1.178457$

SCF Energy $\left(P C M / m P W 1 P W 91 / 6-31+G^{\star *}\right)=-1524.12441179$

S-MTPA-4 001

B3LYP/6-31 G* Geometry

O $2.801195-0.8307090 .831864$

C $4.036953 \quad 1.1666090 .123437$

C $1.6084760 .955225-0.314730$

C $2.7577781 .595626-0.536777$

C $1.518869-0.2711420 .555774$

C $3.763126 \quad 0.143578 \quad 1.228971$

C $5.018645-0.5841061 .616654$

C $6.070537-0.6135020 .792536$

C $6.028304-0.035943-0.574405$

O $4.923193 \quad 0.680673-0.916609$

H 3.3758510 .7026112 .097990

H 4.5324052 .0337030 .583810

C $0.707495-1.418060-0.084719$

C $1.227857-1.880085-1.434362$

O $6.895637-0.232380-1.395096$

H $1.020631-0.0121481 .506518$

O $-0.655131-0.948091-0.288261$

H $0.6784811 .305095-0.756402$

H $2.8051382 .459813-1.194202$

H $5.029676-1.1056542 .570427$ 
H $6.990112-1.135704 \quad 1.035487$

H $0.681809-2.2407220 .633607$

H $2.266686-2.203344-1.329700$

H $1.188930-1.072939-2.171934$

H $\quad 0.628979-2.718253-1.802669$

C $-1.516092-1.0670250 .738668$

O $-1.246795-1.500041 \quad 1.829931$

C $-2.917989-0.4981840 .346165$

O $-3.702470-0.7464291 .490279$

C $-3.491468-1.314661-0.846568$

C $-5.052748-0.278577 \quad 1.519386$

H -5.1424190 .7445381 .138404$

H $-5.338430-0.2977132 .573409$

H $-5.719236-0.9369010 .953401$

$\begin{array}{llll}\text { C }-2.750927 & 1.003681 & 0.064064\end{array}$

C $-2.3474413 .765466-0.253782$

C $-2.5189861 .825315 \quad 1.177715$

C $-2.7724311 .579196-1.211953$

C $-2.5740422 .953394-1.364968$

C -2.3199573 .1955441 .020686$

H -2.501694 1.3782732 .167183

H -2.938850 $0.970414-2.091648$

H -2.599879 $3.386814-2.361041$

H -2.1438043 .8170481 .894456$

H -2.194606 $4.833859-0.379359$

F -2.705343 -1.313826-1.941496

F $-4.694017-0.827798-1.232440$

F $-3.667368-2.594715-0.476660$

SCF Energy $\left(\mathrm{PCM} / \mathrm{mPW} 1 \mathrm{PW} 91 / 6-31+\mathrm{G}^{* *}\right)=-1524.12286516$

S-MTPA-4_002

B3LYP/6-31 G* Geometry

O $2.733217-0.7510270 .877778$

C $3.9929301 .154888-0.017434$

C $1.5705160 .911104-0.470490$

C $2.7270941 .519629-0.739032$

C $1.459369-0.2183700 .519846$

C 3.6952450 .2555731 .185232

C $4.939815-0.433141 \quad 1.667849$

C $6.004741-0.5527850 .869133$

C $5.987596-0.123086-0.551723$

O $4.8920120 .557349-0.985950$

H 3.2979900 .9050901 .983915

H 4.4868012 .0631940 .357115

C $0.658007-1.425075-0.015507$

C $1.207032-2.024419-1.297695$

O $6.866708-0.409275-1.332451$

H 0.9443780 .1389921 .428622

O $-0.700920-0.979379-0.298538$

H $0.6485491 .220883-0.956798$

H $2.7893732 .312760-1.479446$

H $4.932869-0.8509092 .671461$

H $6.917452-1.049747 \quad 1.181110$

H $\quad 0.613435-2.168100 \quad 0.784140$

H $2.247290-2.320139-1.139808$

H $1.173335-1.301770-2.118294$

H $\quad 0.625393-2.905559-1.584380$

C $-1.576890-0.950495 \quad 0.718853$

O $-1.327475-1.250551 \quad 1.863290$

C $-2.958606-0.3916220 .260943$

O $-3.920238-0.547291 \quad 1.276225$

C -3.443155-1.121739-1.023451

C $-4.278133-1.8512111 .752560$

H $-4.978192-2.3439251 .070238$

H $-4.774018-1.6722522 .708921$

H -3.403821 -2.484532 1.915831

C -2.7844701 .1319990 .106562$

$\begin{array}{llll}\text { C }-2.424177 & 3.918721 & 0.047627\end{array}$

C $-2.7056731 .809787-1.115863$

C $-2.672156 \quad 1.864313 \quad 1.298518$

C -2.4930933 .2455721 .268688$

C $-2.5322993 .196016-1.139381$

H $-2.7858001 .277424-2.053581$

H -2.7367391 .3421792 .246422$

H -2.411440 3.7956092 .202256

H -2.485642 $3.707975-2.096722$

H -2.289280 $4.996636 \quad 0.022161$
F -2.699099 -0.885171-2.122052

F $-4.709313-0.775745-1.305664$

F $-3.413871-2.463127-0.827552$

SCF Energy $\left(\mathrm{PCM} / \mathrm{mPW} 1 \mathrm{PW} 91 / 6-31+\mathrm{G}^{* *}\right)=-1524.12204813$

S-MTPA-4_003

B3LYP/6-31G* Geometry

O $2.214908-1.552475-0.747488$

C $3.434253-0.5748531 .151098$

C $1.478518-2.0548601 .511958$

C $2.353698-1.1755752 .002841$

C $1.473219-2.4619550 .058569$

C $3.503693-1.267652-0.214032$

C $4.241731-0.426383-1.215432$

C $4.4124690 .884004-1.016203$

C $3.7997391 .601028 \quad 0.128477$

O 3.1892840 .8540721 .083455

H $4.048349-2.216188-0.059823$

H $4.411733-0.7004581 .638998$

C $0.061183-2.589424-0.535832$

C $0.062381-2.957320-2.013652$

$\begin{array}{lllll}\text { O } & 3.772934 & 2.810593 & 0.204580\end{array}$

H $1.921587-3.472486-0.028074$

O $-0.653104-1.330961-0.427173$

H $\quad 0.723127-2.5058692 .149482$

H $2.321347-0.8717923 .045899$

H $4.604372-0.914297-2.116732$

H $4.9326021 .520609-1.724273$

H $-0.478765-3.3344760 .055967$

H $0.559994-3.921709-2.163940$

H $0.587170-2.197043-2.596353$

H $-0.966407-3.035637-2.376368$

C -1.539347-1.194299 0.573439

O $-1.724571-1.990136 \quad 1.461197$

C -2.340553 0.1389450 .420076

O -2.8328330 .3764161 .722122$

C -3.529689 $-0.186451-0.530101$

C -3.6398961 .5361481 .931282$

H -3.199297 2.4291941 .474678

H -3.680486 1.6660963 .014897

H $-4.656237 \quad 1.394498 \quad 1.548053$

C $-1.431411 \quad 1.267916-0.078124$

C $0.375907 \quad 3.279929-0.832990$

C $-1.4766091 .811710-1.366595$

C $-0.4729151 .745870 \quad 0.827483$

C 0.4268992 .7408170 .454276

C $-0.5794972 .816303-1.736072$

H $-2.1960651 .458670-2.094745$

H $-0.4322991 .324771 \quad 1.827734$

H $1.188603 \quad 3.074357 \quad 1.151007$

H $-0.6284683 .229653-2.740113$

H $1.0872124 .046447-1.126593$

F -3.113770 - $0.726529-1.698610$

F $-4.2534710 .913799-0.835053$

F - $4.362055-1.0670250 .047437$

SCF Energy $\left(P C M / m P W 1 P W 91 / 6-31+G^{* *}\right)=-1524.12055636$

S-MTPA-4 005

B3LYP/6-31G* Geometry

O $-2.907613-1.1317650 .221215$

C $-4.3533880 .322959-1.125606$

C $-2.007006 \quad 0.957422-0.649691$

C $-3.2134901 .301023-1.104211$

C $-1.717307-0.402588-0.069123$

C $-3.857532-1.097371-0.841365$

C $-4.995262-2.003606-0.467097$

C $-6.153585-1.497833-0.032597$

C $-6.348895-0.0459920 .208381$

O $-5.3523720 .792936-0.184691$

H -3.380492 -1.463943 -1.766320

H $-4.8276420 .320757-2.117729$

C $-0.941491-0.3426691 .265060$

C -1.639192 0.4393602 .363648

$\begin{array}{lllll}\text { O } & -7.314684 & 0.396593 & 0.788022\end{array}$

H - $-1.099170-0.978945-0.779940$

O $\quad 0.3304600 .318169 \quad 1.010295$

H - $-1.1719081 .652114-0.690633$ 
H -3.408138 2.297691-1.491497

H $-4.833574-3.076549-0.534096$

H $-6.993782-2.1215810 .254335$

H $-0.738413-1.3702471 .575287$

H -2.619122 -0.0045092 .556931$

H -1.7828241 .4849322 .075435$

H - $-1.0466080 .408976 \quad 3.282763$

C $1.344532-0.4347370 .559420$

O $1.300576-1.6248190 .361487$

C 2.6083580 .4467320 .291952

$\begin{array}{llll}\text { O } & 2.763434 & 1.227449 & 1.460396\end{array}$

C 2.263428 1.367317 -0.919763

C 3.7766922 .2381621 .456053

H 3.8659942 .5596042 .495700

H 3.4835703 .0944980 .839593

H 4.7406781 .8498361 .110160

C $3.812285-0.4647230 .023332$

C $5.984146-2.212105-0.320896$

C $4.161109-0.919602-1.253744$

C $4.551627-0.9096531 .126407$

C $5.631760-1.7742510 .956157$

C $5.242935-1.784281-1.422174$

H $3.594459-0.612619-2.124681$

H $4.272232-0.5765782 .120518$

H $6.195076-2.1071961 .823697$

H $5.502693-2.124973-2.420701$

H $6.826433-2.885101-0.456237$

F $1.6478450 .667487-1.905177$

F $3.3601831 .939757-1.453873$

F $1.4323922 .362082-0.559975$

SCF Energy $\left(\mathrm{PCM} / \mathrm{mPW} 1 \mathrm{PW} 91 / 6-31+\mathrm{G}^{* *}\right)=-1524.12137091$

S-MTPA-4_007

B3LYP/6-31G* Geometry

O $-2.369771-1.6653240 .281843$

C $-3.4776440 .035061-1.090438$

C -1.591324 -1.295725 -1.989855

C $-2.409148-0.248254-2.106990$

C $-1.645656-2.231064-0.807054$

C -3.631331-1.136161-0.116989

C $-4.373514-0.7214931 .121568$

C -4.4603450 .5671151 .465698$

C -3.7403511 .6321250 .726199$

O $-3.1343701 .286723-0.440843$

H $-4.202937-1.920307-0.643594$

H $-4.4448340 .192198-1.588899$

C $-0.258778-2.645238-0.296850$

C $-0.309396-3.5259760 .944666$

O $-3.6154512 .759092 \quad 1.152385$

H - $2.139938-3.173433-1.117496$

O $0.526522-1.473870 \quad 0.061274$

H $-0.838220-1.504686-2.745159$

H -2.328286 $0.438410-2.945185$

H $-4.809614-1.5031821 .738401$

H -4.9785610 .9029552 .357701$

H $\quad 0.257472-3.148502-1.118957$

H $-0.859219-4.4493450 .731639$

H $-0.806359-3.0030621 .764574$

H $\quad 0.706012-3.7873051 .255478$

C $1.511364-1.091165-0.773031$

O $1.697239-1.545150-1.878218$

C $2.419240 \quad 0.006795-0.145144$

O $3.6432430 .042014-0.833367$

C $2.666625-0.2949251 .366929$

C $4.507285-1.095319-0.809293$

H $5.001129-1.2066070 .161667$

H $5.260100-0.892921-1.573999$

H $3.978487-2.017003-1.068345$

C $1.7865141 .403271-0.293850$

C $0.7179983 .986008-0.504867$

C $0.4298391 .631808-0.027635$

C $2.6041922 .478415-0.663851$

C $2.0693003 .761790-0.769801$

C $-0.1008442 .918756-0.135112$

$\begin{array}{llll}\text { H } & -0.221347 & 0.815223 & 0.261501\end{array}$

H $3.6520772 .303041-0.870561$

H $2.7149774 .587196-1.058492$
H - 1.1539143 .0803000 .073582

H $0.3033564 .987400-0.586224$

F $1.609192-0.0205922 .143502$

F 3.7065020 .4317771 .813488

F $2.978323-1.6048731 .561009$

SCF Energy $\left(\mathrm{PCM} / \mathrm{mPW} 1 \mathrm{PW} 91 / 6-31+\mathrm{G}^{* *}\right)=-1524.12402496$

S-MTPA-4_009

B3LYP/6-31G* Geometry

O $-2.8190820 .719772-0.841180$

C $-4.2203240 .573474 \quad 1.168502$

C $-1.806454 \quad 0.0277001 .264355$

C $-3.0059130 .007347 \quad 1.847970$

C $-1.594793 \quad 0.552737-0.132793$

C $-3.825097 \quad 1.377182-0.073113$

C $-5.0078371 .606211-0.970026$

C $-6.0928890 .831653-0.875971$

C $-6.154930-0.347126 \quad 0.024334$

O $-5.120509-0.528727 \quad 0.889925$

H -3.4347542 .3468420 .280505$

H $-4.7505101 .252377 \quad 1.852005$

C $-0.740688-0.383437-1.008473$

C $-1.296005-1.791203-1.160015$

O $-7.043711-1.166252-0.031138$

H -1.062443 $1.520041-0.077553$

O $0.553103-0.412899-0.339468$

H $-0.919804-0.3327691 .779825$

H -3.142614 -0.4059362 .843812$

H $-4.9385782 .398728-1.710946$

H -6.962072 $0.961810-1.512248$

H $-0.6073320 .082472-1.987983$

H $-2.309847-1.737098-1.565989$

H - $1.336491-2.303553-0.194097$

H $-0.671963-2.370163-1.845867$

C $1.642926-0.651677-1.083284$

O $1.645052-0.833444-2.278152$

C $2.940353-0.592449-0.219537$

O $4.034812-1.123282-0.929023$

C $2.732566-1.3871531 .101092$

C $4.020729-2.491198-1.355691$

H $4.235946-3.170517-0.524311$

H $4.822132-2.562082-2.093919$

H $3.074118-2.764420-1.828119$

C $3.2897920 .891958-0.014981$

C 4.0034223 .6036560 .143389

C 3.4111651 .5192211 .229585

C $3.5391101 .634719-1.179312$

C $3.8897762 .980211-1.100780$

C 3.7666982 .8680961 .303239

H 3.2391030 .9750002 .148480

H $3.4644381 .146847-2.145487$

H $4.0784023 .539663-2.012977$

H 3.8614323 .3395462 .277648

H $4.2791994 .652788 \quad 0.207273$

F $1.875024-0.8080531 .969455$

F $3.903098-1.5495591 .740820$

F $2.236844-2.6191060 .833201$

SCF Energy $\left(\mathrm{PCM} / \mathrm{mPW} 1 \mathrm{PW} 91 / 6-31+\mathrm{G}^{* \star}\right)=-1524.12206261$

S-MTPA-4_010

B3LYP/6-31 G* Geometry

O $-2.8632800 .746712-0.807669$

C $-4.2441550 .477984 \quad 1.204505$

C $-1.834910-0.0943651 .235011$

C -3.028118 -0.1441591 .829489$

C $-1.6329550 .524740-0.124720$

C $-3.855673 \quad 1.3583790 .013664$

C $-5.0462621 .655608-0.852192$

C $-6.1380080 .886866-0.795399$

C $-6.202462-0.3466840 .028224$

$\begin{array}{llll}0 & -5.159639 & -0.594633 & 0.866647\end{array}$

H $-3.453606 \quad 2.299528 \quad 0.426617$

H -4.7588001 .1156891 .937831$

C $-0.790947-0.352689-1.070080$

C $-1.356950-1.742940-1.315010$

O $-7.101548-1.150720-0.067912$

H $-1.0956151 .483859-0.007367$ 
O $0.506822-0.434719-0.415892$

H - $0.947056-0.5003821 .712949$

H -3.158798 -0.625709 2.795019

H - $-4.9773262 .493598-1.541334$

H -7.012881 $1.066194-1.411623$

H $-0.6631160 .179292-2.016202$

H $-2.370782-1.654158-1.715038$

H $-1.401350-2.318392-0.385372$

H $-0.737052-2.279236-2.038114$

C $1.585129-0.633496-1.190920$

O $1.572120-0.729748-2.391601$

C $2.891310-0.642403-0.334413$

O $3.844129-1.217896-1.203001$

C $2.710008-1.5839250 .888051$

C $5.229761-1.173176-0.855841$

H $5.763895-1.349919-1.792146$

H $5.492108-1.954985-0.136704$

H $5.521009-0.196990-0.454135$

C 3.2189280 .8155680 .022776

C 3.8541743 .5160650 .463821

C 3.2950791 .3206891 .326109

C $3.4681761 .677953-1.057184$

C $3.7822953 .017161-0.838763$

C $3.6113712 .664406 \quad 1.540426$

H 3.1110000 .6825662 .180956

H $3.4167841 .285516-2.068469$

H $3.9714953 .670136-1.686387$

H 3.6697383 .0412732 .557931

H 4.1001584 .5601370 .637240

F $1.778021-1.172971 \quad 1.776229$

F $3.867757-1.6990831 .580487$

F $2.359403-2.8123340 .471557$

SCF Energy $\left(\mathrm{PCM} / \mathrm{mPW} 1 \mathrm{PW} 91 / 6-31+\mathrm{G}^{\star \star}\right)=-1524.12247977$

S-MTPA-4_011

B3LYP/6-31G* Geometry

$\begin{array}{lllll}\text { O } & 2.678709 & 1.608488 & 0.256439\end{array}$

C $3.900488-0.202476-0.848602$

C $2.0320530 .949857-1.995481$

C $2.897064-0.065734-1.958658$

C $1.9655261 .998251-0.912483$

C $3.9736511 .083455-0.023163$

C $4.6609280 .843233 \quad 1.291397$

C $4.722039-0.3878631 .807812$

C $4.036345-1.544140 \quad 1.179618$

O $3.526436-1.369358-0.070567$

H $4.5470881 .817212-0.615522$

H $4.900094-0.400897-1.260524$

C $0.5271752 .342202-0.491143$

C $0.460048 \quad 3.3820640 .619063$

O $3.862805-2.5952521 .753809$

H $2.3956422 .941172-1.305581$

O $-0.144062 \quad 1.155070 \quad 0.010712$

H $1.3260741 .051730-2.815536$

H $2.903588-0.827986-2.733118$

H 5.0701601 .7011141 .818868

H $5.190816-0.6012322 .762732$

H $-0.0087602 .680688-1.382319$

H $0.941110 \quad 4.3113250 .294184$

H 0.9676243 .0190031 .515348

H $-0.583243 \quad 3.5996750 .864564$

C $-1.0016670 .527752-0.808219$

O $-1.207723 \quad 0.825632-1.965660$

C $-1.779013-0.584557-0.054522$

O $-1.063250-1.1232841 .022726$

C $-2.159283-1.708885-1.068441$

C $0.173517-1.8047820 .776196$

H $0.006894-2.7890910 .329043$

H $\quad 0.632646-1.9305301 .758136$

H $0.845407-1.219186 \quad 0.144212$

$\begin{array}{llll}\text { C } & -3.059879 & 0.047329 & 0.526257\end{array}$

C $-5.3979341 .157767 \quad 1.604791$

C $-3.8663190 .890687-0.249971$

C $-3.433513-0.238121 \quad 1.843909$

C -4.5975630 .3161422 .377653$

C $-5.027792 \quad 1.442607 \quad 0.289792$

H -3.589649 $1.114744-1.274134$
H -2.809244 -0.8889522 .442608$

H -4.8773790 .0864973 .402391$

H $-5.6440042 .095283-0.322758$

H -6.3036511 .5884622 .023516$

F - $3.087884-1.327157-1.959702$

F $-2.643020-2.770820-0.398347$

F $-1.079070-2.127369-1.768687$

SCF Energy $\left(\mathrm{PCM} / \mathrm{mPW} 1 \mathrm{PW} 91 / 6-31+\mathrm{G}^{* *}\right)=-1524.12570333$

S-MTPA-4_013

B3LYP/6-31G* Geometry

O $2.457484-1.497428-0.532001$

C $3.529520-0.0357961 .113028$

C $1.735359-1.6041971 .786086$

C $2.511404-0.560308 \quad 2.084269$

C $1.781152-2.2872160 .441484$

C $3.706855-0.998991-0.062850$

C $4.401899-0.328458-1.213491$

C $4.4247861 .004347-1.311877$

C $3.6708961 .877153-0.379858$

O 3.1046721 .2931030 .710586

H $4.322244-1.8409890 .299305$

H $4.5036550 .079851 \quad 1.609131$

C $0.390007-2.638152-0.106712$

C $0.424750-3.279947-1.487129$

O $3.4837593 .055464-0.590517$

H $2.308924-3.2557930 .551828$

O $-0.432664-1.444779-0.234246$

H $1.021852-1.9946452 .506598$

H $2.437143-0.0589033 .045533$

H $4.858034-0.960511-1.971184$

H $4.9077151 .524547-2.132307$

H $-0.092488-3.2957570 .621753$

H $0.996722-4.213963-1.456408$

H $0.888296-2.607123-2.211833$

H $-0.594842-3.503769-1.812849$

C -1.382871-1.230085 0.694722

O $-1.471557-1.836378 \quad 1.737390$

C $-2.411684-0.1520420 .235833$

O $-3.546178-0.2115091 .073907$

C $-2.976071-0.639014-1.138225$

C -3.426267 0.2939832 .405964

H -2.706743 -0.2849522 .989023$

H -4.4222770 .1811682 .840029$

H -3.148958 1.3538482 .410655

C $-1.847444 \quad 1.2702650 .100645$

C $-0.9767043 .929366-0.126907$

C $-0.482993 \quad 1.5760880 .152710$

C $-2.7731212 .313203-0.067523$

C $-2.3400913 .631822-0.182514$

C -0.0508532 .9010380 .042046$

H $0.2584590 .794203 \quad 0.264439$

H -3.832492 $2.084005-0.102064$

H $-3.069796 \quad 4.426513-0.314156$

H $1.011573 \quad 3.1217510 .084623$

H $-0.6373934 .958064-0.214715$

F -2.111183 - $0.418906-2.140853$

F $-4.124937-0.016738-1.446422$

F $-3.233317-1.968099-1.102343$

SCF Energy $\left(\mathrm{PCM} / \mathrm{mPW} 1 \mathrm{PW} 91 / 6-31+\mathrm{G}^{* *}\right)=-1524.12374149$

S-MTPA-4 014

B3LYP/6-31 G* Geometry

O $-3.106179-0.591127-0.926704$

C $-4.6980681 .028036 \quad 0.003855$

C $-2.270760 \quad 1.246046 \quad 0.439636$

C -3.5195221 .6210340 .722257$

C $-1.9550490 .164264-0.561089$

C $-4.2427210 .220569-1.214201$

C $-5.337383-0.683798-1.704048$

C $-6.351110-1.020856-0.900887$

C $-6.402751-0.6202520 .527889$

$\begin{array}{lllll}O & -5.457088 & 0.254158 & 0.967204\end{array}$

H -3.980369 $0.946384-2.002810$

H $-5.3612021 .828788-0.354218$

C $-0.937257-0.869018-0.040797$

C $-1.359697-1.5959681 .226060$ 
O $-7.201568-1.085048 \quad 1.308979$

H - $-1.5128860 .628129-1.462143$ $\begin{array}{lllll}\text { O } & 0.270809 & -0.098001 & 0.208557\end{array}$

$\mathrm{H}-1.4159821 .7172320 .918031$

H $-3.7257902 .381621 \quad 1.470722$

H $-5.260466-1.074214-2.715724$

H $-7.154415-1.678483-1.216358$

H $-0.730637-1.583672-0.840701$

H -2.320563 -2.0897731 .057459$

H -1.469091 -0.8980922 .061540$

H $-0.615001-2.3523461 .489877$

C $1.450962-0.678669-0.053416$

O $1.601029-1.796172-0.481499$

C 2.6262060 .2993580 .277866

$\begin{array}{llll}\text { O } & 2.407672 & 0.652738 & 1.631804\end{array}$

C $2.4663541 .557246-0.629561$

C 3.2716951 .6370462 .208866

H 4.3275781 .4129822 .023704

H 3.0746581 .5988523 .282221

H 3.0389492 .6399891 .835680

C $3.960305-0.4242900 .060112$

C $6.344535-1.878494-0.243153$

C $4.649785-0.410734-1.158136$

C $4.468874-1.189338 \quad 1.117223$

C $5.654110-1.9076920 .969074$

C $5.835712-1.130899-1.304801$

H $4.2704530 .152268-2.002432$

H $3.926486-1.2221332 .056355$

H $6.034279-2.4939961 .801247$

H $6.359916-1.106422-2.256244$

H $7.268669-2.437778-0.360946$

F $2.1872291 .201682-1.907515$

F $3.5872652 .308072-0.655095$

F $1.4702512 .352104-0.204999$

SCF Energy $\left(P C M / m P W 1 P W 91 / 6-31+G^{\star *}\right)=-1524.12156854$

S-MTPA-4_016

B3LYP/6-31 G* Geometry

O -2.9387140 .2233770 .909351$

C $-4.608036-0.917747-0.482539$

C $-2.214360-0.896908-1.120984$

C $-3.483470-1.139139-1.453814$

C $-1.821005-0.3317570 .219383$

C $-4.068860-0.6460470 .925006$

C $-5.117971-0.0192831 .797729$

$\begin{array}{llll}\text { C }-6.182926 & 0.581966 & 1.258682\end{array}$

C $-6.3402740 .763165-0.206048$

O $-5.4322160 .149882-1.013720$

H $-3.768963-1.6196401 .349221$

H -5.242774 -1.814207 -0.431064

C -0.7943820 .8163370 .129401$

C $-1.2087191 .963613-0.775109$

O $-7.1909931 .474264-0.690224$

H -1.367506 -1.124858 0.839804

O $0.4554730 .282023-0.398610$

H -1.407476 -1.100998 -1.820773

H $-3.748360-1.513439-2.439202$

H $-4.965905-0.0481942 .873703$

H -6.9558481 .0511341 .858463$

H $-0.611358 \quad 1.161437 \quad 1.149495$

H - $2.1741302 .353450-0.441398$

$\mathrm{H}-1.3077321 .635445-1.814114$

H $-0.4666132 .766138-0.732789$

C $1.332281-0.2149730 .490380$

$\begin{array}{llll}\text { O } & 1.127011 & -0.334728 & 1.677807\end{array}$

C $2.692149-0.534830-0.185510$

O $2.553182-0.891901-1.537881$

C $3.398841-1.6784560 .604787$

C $1.844119-2.082127-1.877001$

H $2.444365-2.978266-1.690727$

H $1.639593-2.000079-2.946771$

H $0.894635-2.165483-1.336197$

C $3.5563180 .739423-0.141653$

C $5.1570303 .039516-0.081169$

C 3.6569481 .5001691 .031110

C $4.2652841 .134884-1.281035$

C $5.0607122 .280696-1.247748$
C 4.4528792 .6448851 .057146

H 3.1177891 .2005981 .923060

H $4.1872810 .547230-2.186830$

H $5.6062022 .578454-2.139202$

H $4.5213613 .227207 \quad 1.971903$

H $5.7760723 .932352-0.058757$

F $3.810690-1.3027831 .823154$

F $4.474601-2.100121-0.086372$

F $2.579162-2.7450880 .766809$

SCF Energy $\left(\mathrm{PCM} / \mathrm{mPW} 1 \mathrm{PW} 91 / 6-31+\mathrm{G}^{* *}\right)=-1524.12526957$

S-MTPA-4_024

B3LYP/6-31G* Geometry

O $3.122957-0.964264-0.512701$

C $4.5405800 .040416 \quad 1.224532$

C 2.0712240 .1144461 .400268

C $3.2601500 .538401 \quad 1.831684$

C $1.921011-0.836017 \quad 0.240144$

C $4.279463-1.1524880 .300455$

C $5.445452-1.396281-0.614161$

C $6.344081-0.434178-0.843925$

C $6.1931070 .942739-0.309997$

O $5.1770621 .165244 \quad 0.567933$

H $4.126399-2.0331830 .947756$

H $5.226065-0.2951022 .016223$

C $0.850304-0.394630-0.775622$

C $1.0802890 .983577-1.376337$

O $6.888147 \quad 1.864639-0.671441$

H $1.617378-1.8282890 .623324$

O $-0.399830-0.417477-0.026889$

H $1.1500270 .441276 \quad 1.875977$

H 3.3437381 .2515272 .647569

H $5.515715-2.366283-1.099933$

H $7.193983-0.573143-1.503943$

H $0.791224-1.148849-1.564025$

H $2.0655801 .010227-1.849703$

H $1.0438771 .760017-0.606391$

H $\quad 0.320371 \quad 1.196385-2.132832$

C $-1.523645-0.667323-0.718413$

O $-1.572247-0.913273-1.902132$

C -2.774305 -0.5193360 .187861$

O $-2.503528-0.8164841 .535599$

C $-3.907336-1.445693-0.351401$

C $-2.099336-2.1383041 .890822$

H $-2.940976-2.8376421 .876647$

H -1.715740 -2.056316 2.910176

H $-1.302767-2.5140311 .238811$

C $-3.2326780 .949768 \quad 0.132848$

C -4.0968343 .6150250 .053238$

C $-3.3180241 .629479-1.089750$

C $-3.587627 \quad 1.6108691 .313505$

C $-4.0166542 .937903 \quad 1.270275$

C $-3.7467052 .955883-1.125846$

H -3.052893 $1.125001-2.012309$

H -3.5231931 .0856122 .257909$

H -4.289488 3.4415992 .193763

H -3.806515 $3.472217-2.079949$

H -4.4299054 .6489500 .023339$

F - $-4.410555-1.036004-1.523839$

F $-4.913657-1.4854340 .542279$

F -3.467842 -2.715518 - 0.521377

SCF Energy $\left(\mathrm{PCM} / \mathrm{mPW} 1 \mathrm{PW} 91 / 6-31+\mathrm{G}^{* *}\right)=-1524.12567842$

S-MTPA-4_025

B3LYP/6-31 G* Geometry

O $2.890178-0.657521 \quad 0.839612$

C 4.1258191 .3096950 .048528

C $1.6914261 .092156-0.353816$

C $2.8402661 .719670-0.611770$

C $1.607281-0.1017860 .561737$

C 3.8626560 .3244851 .190607

C $5.119845-0.3966841 .584842$

C $6.161257-0.4563430 .749196$

C $6.1052050 .079340-0.634137$

O $4.997648 \quad 0.788546-0.985489$

H 3.4900450 .9135172 .046253

H 4.6278542 .1904440 .474637 
C $0.781347-1.265102-0.025663$

C $1.278632-1.778969-1.365439$

O $6.962318-0.144241-1.458200$

H 1.1253960 .1918701 .511911

O $-0.585061-0.799517-0.227695$

H $0.758788 \quad 1.425332-0.802984$

H $2.8839972 .556366-1.304065$

H $5.140656-0.8883822 .554154$

H $7.081561-0.9748430 .997195$

H $\quad 0.761190-2.0610040 .722224$

H $2.316411-2.107223-1.265333$

H $1.233371-0.998995-2.131231$

H $0.665866-2.624015-1.692598$

C -1.468166 -0.9790210 .771858$

O $-1.189950-1.372078 \quad 1.879566$

C $-2.915713-0.6235850 .311183$

O $-3.849437-1.125217 \quad 1.242373$

C $-3.201364-1.485585-0.959196$

C $-4.008375-0.4282562 .478086$

H -3.076063 -0.400517 3.048335

H $-4.753148-1.0039563 .031611$

H -4.3837250 .5895752 .320093$

C $-3.0754310 .878914 \quad 0.043145$

C $-3.4672653 .641240-0.323680$

C $-4.1882151 .355379-0.666133$

C $-2.178263 \quad 1.8086170 .584050$

C -2.369676 $3.179108 \quad 0.401199$

C $-4.3765912 .723808-0.853100$

$\mathrm{H}-4.9086850 .656965-1.074648$

H - -1.3257321 .4713291 .165788$

H -1.6610583 .8822630 .830205$

H $-5.2396213 .072543-1.413508$

H $-3.6168294 .707326-0.470493$

F -2.625072 - $0.972522-2.058501$

F $-4.521007-1.589174-1.204500$

F -2.728797 -2.738400 - 0.787873

SCF Energy $\left(\mathrm{PCM} / \mathrm{mPW} 1 \mathrm{PW} 91 / 6-31+\mathrm{G}^{\star \star}\right)=-1524.12302795$

S-MTPA-4 027

B3LYP/6-31 G* Geometry

O $1.728593-0.472783-0.344150$

C $4.153077-0.597595-0.687335$

C $2.764429-2.651350-0.555573$

C $3.971430-2.081880-0.542921$

C $1.491818-1.844205-0.648669$

C $2.8286700 .076831-1.059225$

C $2.865767 \quad 1.551001-0.777753$

C 3.7851272 .0657390 .044562

C 4.7358881 .2226520 .810207

O $4.745070-0.112495 \quad 0.541967$

H $2.677620-0.087026-2.140691$

H $4.874553-0.388258-1.490744$

C $0.400716-2.2995530 .330113$

C $-0.007910-3.7631710 .215588$

O 5.4398171 .6621801 .690764

H $1.076979-1.926701-1.671331$

O $-0.756621-1.4817090 .001761$

H $2.673513-3.733647-0.518449$

H $4.873167-2.680839-0.445827$

H $2.0987942 .170347-1.235212$

H 3.8357143 .1249610 .274564

H $\quad 0.709856-2.0573391 .348825$

H $0.805817-4.4266880 .523071$

H $-0.304161-4.014558-0.808742$

H $-0.858661-3.9527590 .876139$

C $-1.440854-0.9329831 .018591$

O $-1.203118-1.0873012 .189221$

C -2.5717750 .0031010 .483539$

$\begin{array}{lllll}\text { O } & -3.353874 & 0.255613 & 1.631839\end{array}$

C -3.442597 $-0.771057-0.543132$

C $-4.3696361 .259140 \quad 1.583251$

$\mathrm{H}-4.5716141 .5119962 .626656$

H -5.2865330 .8839531 .118479$

H $-4.0355682 .157641 \quad 1.053672$

C $-1.9093371 .274298-0.068148$

C $-0.6562983 .657016-0.871242$

C $-1.9984221 .707551-1.395986$
C - $1.1833252 .046243 \quad 0.852460$

C $-0.561830 \quad 3.2275770 .455150$

C $-1.3736452 .892840-1.791537$

H $-2.5498401 .134908-2.130651$

H $-1.1149191 .713296 \quad 1.883785$

H -0.0060763 .8128051 .182574$

H $-1.4585103 .220162-2.824345$

H $-0.181178 \quad 4.583862-1.182113$

F $-2.788372-1.132430-1.664908$

F $-4.499626-0.022021-0.940184$

F $-3.928185-1.890808 \quad 0.022591$

SCF Energy $\left(\mathrm{PCM} / \mathrm{mPW} 1 \mathrm{PW} 91 / 6-31+\mathrm{G}^{* *}\right)=-1524.12155123$

S-MTPA-4 028

B3LYP/6-31G* Geometry

O $2.907040 \quad 0.9563020 .591949$

C $4.3209900 .157098-1.246581$

C $1.972218-0.599337-1.036559$

C $3.169211-0.735886-1.609855$

C $1.707296 \quad 0.403545 \quad 0.056224$

C $3.8436751 .347043-0.410565$

C 4.9954292 .0205550 .279342

C $6.149165 \quad 1.371944 \quad 0.465137$

C $6.325732-0.058106 \quad 0.107295$

O $5.318365-0.659827-0.581691$

H $3.3594372 .056091-1.103266$

H $4.7904810 .554097-2.158167$

C $0.967734-0.2016161 .270006$

C $1.684419-1.3728781 .918358$

$\begin{array}{llll}\text { O } & 7.286267 & -0.705790 & 0.456855\end{array}$

H $1.0741871 .217570-0.339654$

O $-0.323299-0.6896630 .804189$

H $1.127256-1.209936-1.344230$

H $3.345798-1.488037-2.374185$

H 4.8481423 .0338780 .644422

H 6.9996381 .8217250 .966832

H $\quad 0.793147 \quad 0.604851 \quad 1.985716$

H 2.689421-1.060678 2.213575

H $1.772441-2.2151511 .226052$

H $1.141509-1.7067642 .807872$

C -1.344802 0.1824770 .750396

O $-1.285138 \quad 1.331711 \quad 1.120174$

C -2.617587 -0.5256920 .173328$

O $-2.862380-1.7338970 .865985$

C $-2.293104-0.988516-1.279020$

C -2.949985 -1.636280 2.286374

H - $1.975292-1.3956672 .728723$

H -3.260675 -2.624628 2.630635

H -3.689208 -0.8906082 .600782$

C $-3.849134 \quad 0.389727 \quad 0.122877$

C $-6.1783791 .946045-0.089866$

C $-3.7706241 .736874-0.255882$

C $-5.109298-0.174754 \quad 0.360428$

C $-6.2649250 .599617 \quad 0.264635$

C $-4.9293602 .507302-0.356328$

H -2.810877 $2.189555-0.465472$

H $-5.180652-1.2281720 .607155$

$\begin{array}{llll}H & -7.232880 & 0.145617 & 0.458967\end{array}$

H $-4.8510743 .551876-0.645198$

H -7.077766 $2.551240-0.165990$

F - $-1.8084180 .061539-1.986194$

F $-3.390691-1.440224-1.902160$

F - $1.374436-1.967149-1.328615$

SCF Energy $\left(\mathrm{PCM} / \mathrm{mPW} 1 \mathrm{PW} 91 / 6-31+\mathrm{G}^{* *}\right)=-1524.12324529$

S-MTPA-4 030

B3LYP/6-31 G* Geometry

O $1.665955-0.490461-0.360969$

C $4.086026-0.675679-0.712994$

C $2.624125-2.682319-0.737698$

C $3.850579-2.159001-0.681965$

C $1.382387-1.823957-0.772540$

C $2.7881630 .074345-1.029688$

C $2.8782811 .519200-0.632593$

C 3.8160461 .9341560 .224756

C $4.735947 \quad 1.0004510 .919197$

O $4.695227-0.309807 \quad 0.548568$ 
H $2.6337290 .002107-2.120557$

H $4.815080-0.432376-1.499861$

C $0.266574-2.3135910 .161518$

C $-0.196338-3.745409-0.077087$

$\begin{array}{lllll}\text { O } & 5.456858 & 1.343594 & 1.828271\end{array}$

H $0.971641-1.811947-1.800150$

O $-0.862857-1.435129-0.107294$

H $2.494203-3.760376-0.782334$

H $4.730012-2.795533-0.629417$

H $2.1345492 .200099-1.037802$

H 3.9045672 .9696670 .536566

H $\quad 0.574357-2.162664 \quad 1.197995$

H $0.586350-4.4628360 .186632$

H $-0.483930-3.901917-1.122565$

H $-1.066515-3.9554470 .551335$

C $-1.440752-0.8057610 .926753$

O $-1.141061-0.9399872 .089573$

C -2.5403320 .1881370 .439452$

$\begin{array}{lllll}\text { O } & -3.320670 & 0.637841 & 1.522758\end{array}$

C $-3.451183-0.503973-0.612178$

C $-4.087056-0.2924732 .297187$

H $-4.999871-0.5929841 .771940$

H $-4.3583320 .254977 \quad 3.202281$

H -3.505112 -1.174571 2.574423

C $-1.8313041 .452954-0.076011$

C $-0.5295123 .838430-0.787606$

C $-1.9546721 .965914-1.371707$

C $-1.0509782 .147890 \quad 0.860128$

C $-0.404608 \quad 3.3301430 .507710$

C $-1.3050323 .152215-1.721401$

H -2.555557 $1.462508-2.117299$

H -0.9622001 .7598931 .869195$

H 0.1914903 .8561891 .248366

H - $1.4181533 .542196-2.729409$

H $-0.0352134 .766577-1.062808$

F -2.848844 - $0.748431-1.792143$

F $-4.538750 \quad 0.247270-0.859889$

F -3.880947 -1.701284-0.141657

SCF Energy $\left(\mathrm{PCM} / \mathrm{mPW} 1 \mathrm{PW} 91 / 6-31+\mathrm{G}^{* *}\right)=-1524.12101327$

S-MTPA-4_037

B3LYP/6-31 G* Geometry

O 2.9296820 .5830891 .008997

C $4.2414950 .858782-1.048726$

C $1.8106870 .393922-1.148412$

C $2.9840120 .478915-1.777189$

$\begin{array}{llll}\text { C } & 1.673767 & 0.605811 & 0.338367\end{array}$

C $3.9245491 .371903 \quad 0.358638$

C 5.1507791 .3697731 .225609

C $6.2089840 .618427 \quad 0.906574$

C $6.199677-0.327082-0.238074$

O $5.121546-0.293321-1.067933$

H $3.5511942 .404627 \quad 0.248349$

H $4.7600851 .664203-1.588756$

C $0.821113-0.4770301 .026303$

C $1.336494-1.8959130 .837372$

O $7.068921-1.150108-0.413447$

H $1.177776 \quad 1.5781330 .518770$

$\begin{array}{llll}\text { O } & -0.496098 & -0.327671 & 0.419317\end{array}$

H $\quad 0.8945800 .165308-1.686991$

H $3.0681960 .287660-2.843704$

H 5.1361941 .9752092 .128463

H 7.1084600 .5872421 .512596

H $0.739785-0.2353642 .089253$

H $2.373852-1.9495901 .178713$

H $1.301396-2.186349-0.216784$

H $\quad 0.734481-2.597841 \quad 1.419485$

C $-1.559775-0.807418 \quad 1.087704$

O $-1.518824-1.2574462 .207203$

C $-2.856659-0.7299590 .224896$

O $-3.821879-1.621845 \quad 0.737465$

C $-2.530098-1.351529-1.170989$

C $-4.474179-1.2900931 .967838$

H $-3.768130-1.2715722 .799829$

H $-5.203458-2.0885282 .120832$

H $-5.000382-0.3313501 .897677$

C $-3.3959650 .698502 \quad 0.054145$
C $-4.5085043 .257816-0.279625$

C $-4.6659400 .856409-0.522868$

C $-2.6961941 .837374 \quad 0.466347$

C -3.2499053 .1086910 .300463$

C $-5.2152792 .125649-0.690613$

H $-5.221166-0.021082-0.834739$

H -1.7119541 .7451090 .911156$

H -2.692630 3.9819920 .628334

H $-6.1984812 .229104-1.141434$

H -4.937902 $4.247586-0.408349$

F - $1.799193-0.516315-1.935391$

F $-3.651094-1.636239-1.853537$

F - $1.835605-2.498977-1.030194$

SCF Energy $\left(\mathrm{PCM} / \mathrm{mPW} 1 \mathrm{PW} 91 / 6-31+\mathrm{G}^{\star *}\right)=-1524.12359820$

S-MTPA-4_040

B3LYP/6-31 G* Geometry

O $1.943180-0.298352-0.396192$

C $4.371389-0.440075-0.652594$

C $2.980375-2.485858-0.473015$

C $4.187253-1.916843-0.449978$

C $1.708389-1.686950-0.629022$

C $3.0642350 .205352-1.118417$

C $3.1019861 .695874-0.940611$

C $3.9719232 .257656-0.095467$

C 4.8632561 .4563650 .781166

$\begin{array}{lllll}O & 4.890921 & 0.109729 & 0.582488\end{array}$

H $2.945139-0.035614-2.188478$

H $5.131845-0.262228-1.426815$

C $0.609063-2.1067010 .355598$

C $0.222990-3.5810600 .294494$

O $\begin{array}{lllll}5.503807 & 1.944200 & 1.683680\end{array}$

H $1.308777-1.822041-1.651381$

O $-0.549770-1.308539-0.021810$

H $2.887496-3.565551-0.396050$

H $5.084834-2.512214-0.305451$

H $2.3866522 .291963-1.501835$

H 4.0255753 .3296020 .063930

H $0.903536-1.8216301 .368229$

H $1.035932-4.2171970 .656437$

H $-0.036622-3.880202-0.726786$

H $-0.643167-3.7535740 .937711$

C - $1.440446-1.024850 \quad 0.944567$

O $-1.326748-1.3633182 .098948$

C $-2.659983-0.2240200 .395340$

O $-3.737025-0.3266341 .301790$

C $-3.201386-0.982409-0.856341$

C -3.6615990 .3886632 .539648$

H -2.8457970 .0198793 .163593$

H -4.617935 0.1962213 .031004

H $-3.552573 \quad 1.4665172 .375490$

C -2.325586 1.2305750 .029243

C $-1.8597043 .927991-0.603222$

C -1.018192 $1.724641-0.041848$

C $-3.3973982 .101333-0.223513$

C $-3.1658543 .437956-0.540392$

C $-0.792046 \quad 3.067877-0.352467$

H $-0.167267 \quad 1.0740250 .124454$

H $-4.4126071 .725011-0.164369$

H $-4.0074874 .097498-0.733934$

H $0.2285383 .439939-0.389490$

H -1.678017 $4.972265-0.842883$

F -2.406102 - $0.816785-1.926960$

F $-4.428164-0.551081-1.195583$

F -3.290597-2.306854-0.606749

SCF Energy $\left(\mathrm{PCM} / \mathrm{mPW} 1 \mathrm{PW} 91 / 6-31+\mathrm{G}^{\star *}\right)=-1524.12412275$

S-MTPA-4_046

B3LYP/6-31 G* Geometry

$\begin{array}{llll}\text { O } & 2.283070 & 0.344709 & 0.500370\end{array}$

C 4.6684960 .9067600 .505102

C 2.9480792 .6330650 .046385

$\begin{array}{llll}\text { C } & 4.230516 & 2.264791 & 0.037367\end{array}$

C 1.8354661 .6997020 .458180

C $3.5149460 .177108 \quad 1.198169$

C $3.789413-1.2957621 .299616$

C $4.693169-1.8773160 .505367$ 
C $5.397115-1.142275-0.575348$

O $5.2095780 .205163-0.640271$

H 3.4214490 .6073562 .210099

H 5.4868791 .0099181 .232523

C $0.6430291 .717009-0.508728$

C $0.0340163 .091405-0.763297$

O $6.067651-1.696455-1.415409$

H $1.4599871 .992946 \quad 1.456473$

$\begin{array}{llll}\text { O } & -0.367002 & 0.884479 & 0.127138\end{array}$

H $2.6714963 .646380-0.231541$

H $5.0079022 .948950-0.292215$

H $3.212451-1.8721842 .018471$

H $4.910227-2.9395890 .545731$

H $0.9370081 .240645-1.446363$

H $0.7184843 .728266-1.331258$

H -0.2239213 .5931660 .175800$

H $-0.8785722 .976362-1.354230$

C $-1.1108650 .107172-0.674616$

O $-1.0198620 .058637-1.880754$

C $-2.177078-0.670506 \quad 0.141201$

O $-1.787802-0.9077601 .470140$

C $-2.475305-2.022843-0.577548$

C $-0.616066-1.6845971 .730544$

H $-0.777950-2.7455051 .516587$

H - $0.430277-1.5596402 .800028$

H $0.250260-1.3158401 .172266$

C -3.456246 0.1866520 .194306

$\begin{array}{llll}\text { C }-5.798587 & 1.723455 & 0.314475\end{array}$

C $-3.9605820 .799822-0.960875$

C $-4.134520 \quad 0.3450951 .407611$

C $-5.299606 \quad 1.110828 \quad 1.464136$

C $-5.1250711 .564559-0.897391$

H $-3.4476610 .677527-1.908300$

H $-3.745498-0.1291082 .299589$

H $-5.817741 \quad 1.2261132 .412493$

H -5.505368 $2.034638-1.800247$

H $-6.7059332 .319701 \quad 0.361847$

F $-3.123832-1.872753-1.741429$

F $-3.234177-2.7961380 .221968$

F $-1.334238-2.703891-0.835583$

SCF Energy $\left(P C M / m P W 1 P W 91 / 6-31+G^{* *}\right)=-1524.12526740$

S-MTPA-4_047

B3LYP/6-31G* Geometry

O $-2.673997 \quad 1.247077-0.807247$

C $-3.829342-0.0878310 .892970$

C $-2.084067 \quad 1.550575 \quad 1.531579$

C -2.8691820 .5267991 .871275$

$\begin{array}{lll}\text { C }-2.053921 & 2.119167 & 0.134295\end{array}$

C $-3.9451290 .771332-0.368242$

C $-4.563880-0.001683-1.497711$

C $-4.559053-1.338095-1.488494$

C $-3.856829-2.121772-0.441434$

O $-3.377703-1.4421200 .635161$

H - $4.585776 \quad 1.633635-0.115068$

H $-4.829742-0.166603 \quad 1.342171$

C $-0.6300152 .416918-0.362496$

C $-0.5870132 .926800-1.796486$

O $-3.641533-3.308142-0.546546$

H -2.583949 3.0922240 .128756

O $0.1683951 .204072-0.318682$

H -1.4162842 .0058372 .258510$

H -2.842784 0.1053302 .872376

H $-4.9850430 .558720-2.328600$

H $-4.985366-1.931547-2.290560$

H $-0.180873 \quad 3.1377970 .325918$

H -1.168806 $3.850991-1.884463$

H - $1.0040022 .185264-2.481351$

H $0.4454363 .139193-2.088473$

C $1.033348 \quad 1.0498520 .703497$

$\begin{array}{lllll}\text { O } & 1.200233 & 1.857818 & 1.588690\end{array}$

C $1.784863-0.3048610 .575100$

O $0.888686-1.3984120 .603545$

C $2.614724-0.5227981 .874798$

C $-0.005377-1.638264-0.491212$

H $-0.836044-0.931597-0.494159$

H $-0.387929-2.647202-0.323581$
H $\quad 0.512870-1.594555-1.454696$

C $2.740687-0.302166-0.623701$

C $4.573994-0.357226-2.745966$

C $3.2758300 .887714-1.129467$

C $3.149421-1.522407-1.175539$

C $4.055569-1.548361-2.235063$

C $4.1861110 .860622-2.186233$

H $2.9980591 .842018-0.690506$

H $2.760578-2.447486-0.763992$

H $4.360478-2.501969-2.657432$

H $4.5937831 .792548-2.568410$

H $5.280344-0.378219-3.571442$

F 3.5575160 .4269782 .010751

F $3.241956-1.7140541 .829918$

F $1.840304-0.5143262 .970088$

SCF Energy $\left(\mathrm{PCM} / \mathrm{mPW} 1 \mathrm{PW} 91 / 6-31+\mathrm{G}^{* *}\right)=-1524.12317634$

S-MTPA-4 057

B3LYP/6-31G* Geometry

O -2.959396 -0.792074-0.740782

C $-4.428817 \quad 1.092040-0.184068$

C $-2.038563 \quad 1.1185180 .461799$

C -3.2558871 .6542320 .567370$

C $-1.764878-0.116795-0.355191$

C $-3.966567 \quad 0.085585-1.240763$

C $-5.110902-0.761575-1.719230$

C $-6.219756-0.884137-0.983292$

C $-6.345033-0.293487 \quad 0.372972$

O

H $-3.5562410 .666565-2.083961$

H $-4.9648421 .900153-0.702320$

C $-0.932673-1.1742380 .404504$

C -1.562466 -1.656376 1.699022

O $-7.251506-0.5702261 .125508$

H -1.192642 $0.163854-1.256562$

O $0.343909-0.559693 \quad 0.745094$

H -1.179794 1.5664990 .955086

H $-3.4352732 .530571 \quad 1.184593$

H - $-4.993706-1.289696-2.662171$

H -7.062433 -1.494391-1.291215

H $-0.744983-2.004458-0.279918$

H -2.550790 -2.070922 1.484800

H - $1.679861-0.8354182 .412515$

H $-0.944574-2.4353142 .156450$

C $1.309622-0.578863-0.186315$

O $1.222030-1.140017-1.256055$

$\begin{array}{llll}\text { C } & 2.526037 & 0.302537 & 0.232647\end{array}$

O $2.386726 \quad 0.8282551 .535350$

C $2.3940201 .571478-0.668644$

C $2.554989-0.0733202 .629759$

H $1.831807-0.8937192 .590886$

H 2.3701150 .5250213 .524290

H $3.571800-0.4785602 .667568$

C $3.898996-0.3521800 .002878$

C $6.482662-1.411761-0.322868$

C $4.097777-1.519434-0.743556$

$\begin{array}{llll}\text { C } & 5.012460 & 0.283324 & 0.576737\end{array}$

C $6.292430-0.2423520 .415670$

C $5.382754-2.044249-0.899775$

H $3.258415-2.012350-1.216398$

H 4.8680801 .1907531 .152631

H $7.140876 \quad 0.263697 \quad 0.868265$

H $5.518411-2.952186-1.481170$

H $7.480325-1.823685-0.448614$

F $2.6269501 .279395-1.955863$

F $3.2493322 .534127-0.295613$

F $1.1425022 .084152-0.584153$

SCF Energy $\left(P C M / m P W 1 P W 91 / 6-31+G^{* *}\right)=-1524.12379461$

S-MTPA-4 060

B3LYP/6-31 G* Geometry

O $2.925876-0.795341-1.139161$

C 3.8956880 .0435950 .946941

C $2.410632-1.9324650 .953969$

C $3.069218-0.9999341 .641717$

C $2.393866-1.980586-0.553567$

C $4.123107-0.333398-0.517724$ 
C $4.6162010 .847630-1.305895$

C $4.3845762 .091026-0.873699$

C 3.5501682 .3685340 .322464

O 3.2190411 .3163571 .115379

H $4.879809-1.136113-0.534088$

H 4.8784130 .1369541 .430591

C $0.971807-2.162615-1.102132$

C $0.887985-2.111259-2.622270$

O 3.1097523 .4689720 .571714

H $2.980061-2.853893-0.899993$

O $0.188976-1.093433-0.508679$

H $1.835979-2.6992071 .469594$

H $3.024615-0.9625792 .726522$

H $5.1304940 .659157-2.244883$

H $4.7049132 .972011-1.420023$

H $0.571194-3.112935-0.734070$

H $1.514287-2.898239-3.057504$

H $1.237627-1.144997-2.992598$

H $-0.143229-2.277111-2.942484$

C $-1.125385-1.292927-0.356182$

O $-1.717538-2.276842-0.744455$

C -1.797888 -0.1286760 .433137$

$\begin{array}{lllll}\text { O } & -0.870914 & 0.780063 & 0.980456\end{array}$

C -2.396123 -0.8059951 .707091$

C -0.1825621 .6907830 .114562$

H $0.5256151 .166040-0.529947$

H $\quad 0.3681272 .357150 \quad 0.780526$

H $-0.8821512 .275829-0.492028$

C $-2.9274590 .560402-0.352858$

C $-5.0120201 .918782-1.659885$

C $-3.400324 \quad 1.7875380 .137947$

C $-3.5202890 .016238-1.497902$

C $-4.5531310 .695966-2.147215$

C $-4.4327562 .461237-0.511271$

H -2.952669 2.209840 1.030801

H $-3.193621-0.944200-1.877580$

H -5.001236 $0.259967-3.036107$

H $-4.7841073 .411073-0.117266$

H $-5.8163482 .444153-2.167858$

F -3.417201-1.620153 1.401855

F -2.846189 0.1102452 .578992

F -1.453407 -1.541690 2.337263

SCF Energy $\left(P C M / m P W 1 P W 91 / 6-31+G^{* *}\right)=-1524.12318122$

S-MTPA-4_104

B3LYP/6-31 G* Geometry

O $-2.017083-0.013034-1.271618$

C $-4.431156-0.000083-0.937479$

C $-3.1524292 .128358-0.886348$

C $-4.3239451 .496445-0.823065$

C $-1.8551161 .401181-1.113090$

C $-3.091271-0.666054-0.585920$

C $-2.912849-0.7484360 .908675$

C $-3.969972-0.7284401 .728104$

C $-5.358327-0.6643891 .219760$

O $-5.524183-0.489633-0.129235$

H $-3.065404-1.684544-0.993232$

H $-4.721502-0.275236-1.956997$

C $-0.7794081 .715933-0.048591$

C -0.4395683 .1968890 .073559$

O $-6.334384-0.8164931 .916545$

$\mathrm{H}-1.434028 \quad 1.729384-2.074601$

O $0.4330161 .045365-0.492271$

H $-3.1037213 .211781-0.818768$

H $-5.2579052 .039385-0.700290$

H $-1.899256-0.8729931 .282010$

H $-3.878829-0.8237432 .804987$

H $-1.067025 \quad 1.302407 \quad 0.918944$

H -1.2748043 .7537760 .508358$

H $-0.1945323 .629936-0.902664$

H 0.4281613 .3215800 .727509

C 0.8901620 .0072520 .218457

O $0.398773-0.422461 \quad 1.240710$

C $2.211954-0.531375-0.389805$

O $2.262076-0.384570-1.784888$

C $2.379350-2.033149-0.001741$

C $1.286828-1.053920-2.590897$
H $1.512457-2.120680-2.687169$

H $1.358024-0.581343-3.573029$

H $0.271295-0.924222-2.203040$

C 3.3749910 .2902330 .196304

C $5.511387 \quad 1.772108 \quad 1.243638$

C 3.4445230 .5568701 .570474

C $4.3832190 .766122-0.648423$

C $5.4451641 .503764-0.123827$

C 4.5077321 .2958632 .088283

$\begin{array}{llll}\text { H } & 2.672706 & 0.186511 & 2.236298\end{array}$

H $4.3284740 .559405-1.709687$

H $6.2230201 .868126-0.789536$

H 4.5497731 .4965293 .155356

H 6.3393762 .3475891 .648828

F $2.613522-2.2181901 .306070$

F $3.413521-2.556533-0.685769$

F $1.277445-2.752842-0.319817$

SCF Energy $\left(\mathrm{PCM} / \mathrm{mPW} 1 \mathrm{PW} 91 / 6-31+\mathrm{G}^{* *}\right)=-1524.12209122$ 